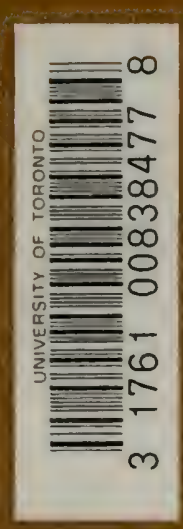



Digitized by the Internet Archive in 2008 with funding from Microsoft Corporation 



HEREDITY AND EUGENICS 
THE UNIVERSITY OF CHICAGO PRESS CHICAGO, ILLINOIS

Egents

THE BAKER \& TAYLOR COMPANY

NEW YORK

THE CAMBRIDGE UNIVERSITY PRESS

LONDON AND EDINBORGE 


\section{HeREDity AND Eugenics}

A COURSE OF LECTURES SUMMARIZING RECENT ADVANCES IN KNOWLEDGE IN VARIATION, HEREDITY, AND EVOLUTION AND ITS RELATION TO PLANT, ANIMAL, AND HUMAN IMPROVEMENT AND WELFARE

BY

WILLIAM ERNEST CASTLE JOHN MERLE COULTER CHARLES BENEDICT DAVENPORT EDWARD MURRAY EAST WILLIAM LAWRENCE TOWER
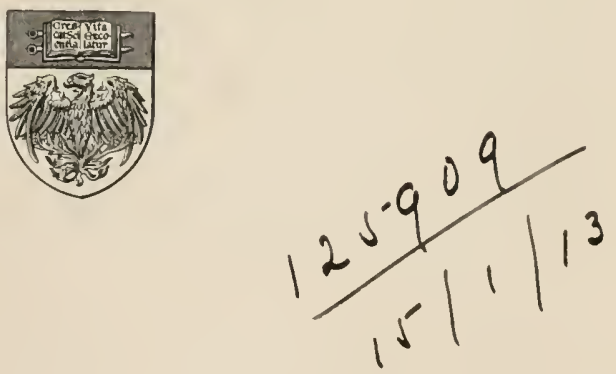

THE UNIVERSITY OF CHICAGO PRESS CHICAGO, ILLINOIS 


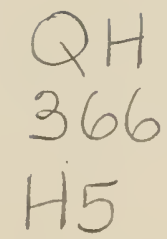

COPYRIGHT I9I2 By

The University of Chicago

All Rights Reserved

Published June Igr2

No 


\section{PREFACE}

During the summer of I9II, a course of lectures on heredity and allied topics was given at the University of Chicago, under the auspices of the biological departments. The purpose of the course was to present the recent developments of knowledge in reference to variation, heredity, and evolution, and the application of this new knowledge to plant, animal, and human development and improvement.

The lectures were not intended for those trained in biology, but for a general university audience, interested in the progress of genetics as a matter of information rather than of study. The lecturers, therefore, did not address themselves to their colleagues, and did not attempt to include any considerable amount of new material. It is believed that a much larger audience than the one originally addressed might be interested in this summary of results in one of the important and recently cultivated fields of biology, and therefore this volume has been published. It is hoped that it may perform a service not only for those interested in biology as a field outside their own experience, but also for those biologists whose work deals with other phases of biology.

The lectures were given by five lecturers, with no opportunity to relate the lectures to one another other than as suggested by the assigned titles. It is inevitable that there should be more or less overlapping of statements, and no attempt has been made to avoid this. Each lecture, therefore, is complete in itself, as it was delivered. 
No attempt has been made to include the whole of the fields represented by the general topics. The plan was to select certain representative investigators to speak of their work. Four such investigators were selected, the mission of the fifth lecturer being to give the elementary information (chaps. $\mathrm{i}$ and ii) necessary for an audience untrained in biology, and thus to prepare the way for the more special topics.

It is hoped that similar series of lectures in other fields of biology will be given during successive summers, and that the present volume may be the first of a series which will represent the most significant aspects of current biological investigation.

\author{
J. M. Coulter \\ F. R. LILLIE \\ W. L. TOWER
}




\section{TABLE OF CONTENTS}

CHAPTER

PAGE

I. Recent Developments in Heredity and Evolution: GeNERAL InTRODUCTION . . . . . . . 3

II. The Physical Basis of Heredity and Evolution from the Cytological Standpoint . . .

John Merle Coulter, Professor and Head of the Department of Botany, the University of Chicago

III. The Method of Evolution . . . . . . 39

IV. Heredity and Sex . . . . . . . . 62

William Ernest Castle, Professor of Zoölogy, Harvard University

V. Inheritance in the Higher Plants

VI. The Application of Biological Principles to Plant BREEDING . . . . . . . . . . II3

Edward Murray East, Assistant Professor of Experimental Plant Morphology, Harvard University

VII. Recent Advances and the Present State of KnowlEDGE CONCERNING the MODification OF thE GerMinal Constitution of Organisms by Experimental PROCESSES . . . . . . . . . . I4I

William Lawrence Tower, Associate Professor of Zoölogy, the University of Chicago

VIII. The Inheritance of Physical and Mental Traits of Man and Their Application to Eugentcs . . 269

IX. The Geography of Man in Relation to Eugenics . 289

Charles Benedict Davenport, Station for Experimental Evolution, Carnegie Institution of Washington INDEX 



\section{JOHN MERLE COULTER}

Professor and Head of the Department of Botany The University of Chicago 



\section{CHAPTER I}

\section{RECENT DEVELOPMENTS IN HEREDITY AND EVOLUTION: GENERAL INTRODUCTION}

This series of lectures is intended to present, in outline, the recent development of knowledge in reference to heredity and evolution. These subjects have to do, not only with the most fundamental conceptions of biology, but they have come to be of immense practical importance in animal and plant breeding. From every aspect, therefore, they appeal to all persons intelligent enough to be interested in the progress of knowledge and in human welfare. At the same time, it is recognized that most people are denied the opportunity of knowing the progress that has been made in these subjects, through lack of biological training or lack of time. To them the suggestions of progress have come chiefly through ephemeral and often misleading publications.

It is the purpose of this series, therefore, to present this information in such a form that it can be appreciated by those who have no special training in biological work; in short, to interpret the significant results of recent investigations.

Before presenting the recent developments in the investigation of heredity and evolution, it is essential to provide a historical background, for nothing is more obvious than that the work of today has evolved gradually from all the work of the past. It should be understood, also, that the subject is so vast in scope and in work that to outline it in a few lectures will require the most rigid selection of material, a selection so rigid that students of the subject will be able to point out glaring omissions. 
If rigid selection is necessary in presenting the recent work, it must be still more rigid in sketching the historical background, with its enormous literature, stretching through many years. Probably no two biologists would put the same details into the background, but probably all of them would give the background the same general aspect; for it is more of an atmosphere than detail that is needed. It will be an aid to understanding and to memory if this sketch is broken up into distinct topics, if it be understood clearly that there are no such natural lines of cleavage in the subject.

\section{THE CONCEPTION OF EVOLUTION}

Those who know of the theory of evolution only in a superficial way, as a thing heard of rather than understood, almost invariably associate it with some man who stands to them as its author. In my own experience, I have encountered a widespread conviction that Darwin is responsible for the theory of organic evolution. The fact is that the conception of evolution, both inorganic and organic, is as old as our record of man's thought, and therefore no one is responsible for it. It is the common property of the human race.

However, a sharp distinction must be made between the speculative stage of evolution and the observational stage. The former is imaginative or philosophical, and could not establish evolution as a fact; the latter is scientific, and has established evolution as a fact. In a real sense, therefore, organic evolution as a definite working principle is comparatively modern, being but little more than one hundred years old. 


\section{THE FACT OF EVOLUTION}

It may be helpful to indicate some of the things that began to open the eyes of thinking men and finally compelled them to accept organic erolution as a fact.

I. The growing proof that the inorganic world had been formed by a process of slow evolution rather than by a series of miraculous catastrophes, compelled the suggestion that the organic world may have developed in the same gradual way by natural processes.

2. The observed intergrading of species, frequently so complete as to make distinct boundaries for species impossible, strongly suggested the passing of one into the other. Dr. Asa Gray once remarked that he did not believe there are any species of North American asters, although he had been studying them for twenty years. Of course this was an expression of despair rather than of belief, but it illustrates the situation. Botanists for a long time emphasized the boundaries of species by preserving in their herbaria what they called "typical specimens" and discarding the intergrades, so that in turning over the sheets of a herbarium the species looked quite distinct; but any excursion into the field brought trouble.

3. Observations began to multiply showing that plants and animals are often able to respond to changed conditions and change their own form or structure. This was called the power of "adaptation," and it has been a most persistent idea. The fact of change was evident, but its explanation has been outgrown. But taking it as a fact, it was evident that the small changes observed would suggest the possibility of indefinitely extended changes. 
4. More intimate knowledge of the structure of plant and animal bodies revealed what were called "rudimentary" structures, which are quite evidently abandoned structures. This suggested at once that they were functioning structures in the ancestral forms. Even man, and perhaps man most of all, was recognized as being a walking museum of antiquity.

5. Then the life panorama of the geological record began to be unrolled; and it became clear that a fauna and a flora totally unlike that of today existed in the earliest periods; that as one approached the later records, resemblances began to appear; and that insensibly the fauna and flora of the ancient world merged into those of today. This was historical evidence of tremendous weight in favor of the fact of a gradual organic evolution.

6. Soon what is called embryology began to be studied, and plants and animals were traced, stage by stage, from the egg to the adult form. In the course of this development resemblances to other forms appeared, which had disappeared when the adult stage was reached. And so the idea developed that here were glimpses of earlier connections, and it became formulated in the well-worn statement that the history of the individual repeats the history of the race, a theory labeled "recapitulation."

7. Men's eyes also began to be opened to the fact that great changes had been wrought in plants by cultivation, and in animals by domestication; so great in many cases that the wild originals could not be recognized with certainty. Later, Darwin called this "an experiment upon a gigantic scale," but it was an experiment unconsciously performed. At least it proved that the operations of man could modify plants and animals, and modify them so much that resemblances to the wild originals would be obscured. 
The seven categories of facts thus indicated, and others that might be added to them, will explain why a number of scientific men were so impressed by the idea that organic evolution is a fact, that they thought it important to search for an explanation of the process.

\section{THE EXPLANATION OF EVOLUTION}

To accept organic evolution as a fact, and to explain it as a process are two very different things, and must be kept clearly distinct. The failure to distinguish them has led recently to much confusion in popular statement and belief. For example, the more exact work of recent years has developed a considerable body of criticism against Darwin's theory of natural selection. To those who thought of the theory of organic evolution as belonging to Darwin, these criticisms seemed to indicate that belief in organic evolution was tottering; when in fact, if any belief was tottering, it was a belief in natural selection as a sufficient explanation of the process of evolution. Darwin's explanation, Lamarck's explanation, every explanation hitherto proposed, may be found inadequate, and still organic evolution will remain to be explained. It must be remembered that the work of biologists has been to explain the fact of organic evolution, not to propose it as an idea; and the destruction of no explanation can weaken the fact.

A single address does not permit the mention of all the proposed explanations of organic evolution, but a few dominating ones may be selected as illustrations. The selection is made with a full appreciation of the fact that professional biologists may think that others should be included.

It is important to distinguish between the two methods of attacking the problem. The earlier method, and the 
one that prevailed for nearly one hundred years, was observational. Series of intergrading plants and animals were observed, and by comparing them it was inferred that they represented the series of transformations that had occurred actually in nature. This has remained the only possible method for the paleontologist, and he also has the advantage of dealing with series of enormous length. It is to be expected, therefore, that the paleontologist will be impressed most strongly by those explanations of evolution which have been derived from observation and comparison.

The later method of attacking the problem, a method that has developed with great rapidity during the last decade, is experimental. Plants and animals are taken in hand and are made to show their possibilities.

It should be kept in mind that the problem is to explain how one species can produce another. The study of organic evolution deals only with the succession of forms, with the production of new forms by previously existing ones. It has nothing to say concerning origins. How the numerous series of living forms may have originated is certainly beyond the reach of biological science as yet. When one goes beyond the observed changes, and tries to trace the successions back to their source, he is in a region of speculation, and outside the boundaries of science. One may stand beside a great stream and discover that its waters are moving; he also recognizes the direction of the movement; but he can know nothing of the distant sources of the stream, for he sees only a very small section. So the scientific recognition of organic evolution simply observes the movement and its direction. The sources are far too distant for observation, and the possibilities are too numerous for profitable speculation. It is evident that 
people in general are more interested in speculation than in plain facts; and they are so interested in such speculations as the origin of life and the origin of man that they come to believe that these speculations belong to the scientific study of organic evolution. But we are simply collecting the facts of change and trying to discover the causes and processes of change in the plants and animals that can come under our observation. IVe can thus discover laws of evolution, just as we discover the law of gravitation, by observing them in operation. Of course the ultimate questions continually suggest themselves, but it must not be thought that any proposed answers to them are a part of biological science.

I. Environment.-The first attempt at what might be called a scientific explanation of organic evolution, because based upon observation, was that it is caused by changes in environment. This explanation began to take definite form during the last decade of the eighteenth century, in the writings of such observers as Erasmus Darwin of England, St. Hilaire of France, and Goethe of Germany. Environment is a term quite variable in its biological application, but we do not need to discuss it in this connection. These older observers saw changes occurring in plants and animals (especially the latter), in response to changes in seasons, in exposure, in climate, etc.; and their picture of the process of evolution was that plants and animals are plastic organisms that are being molded by their environment. The environment and the molding were not analyzed, but thought of in a very superficial sense; so that it was not long before it was recognized that the changes thus induced are too superficial and ephemeral to furnish an adequate explanation of evolution. But it must not be forgotten 
that environment, even in its superficial sense, is a very real factor, and has played its part in every evolutionary theory since.

2. Use and disuse.-In the early part of the nineteenth century, the first substantial explanation of organic evolution was proposed. Its author was Lamarck, and the theory has become styled Lamarckism or Lamarckianism, but its author called it "appetency," or the doctrine of desires. It is more intelligible to the uninformed as the effect of use and disuse. This explanation has been a conspicuous part of evolutionary doctrine ever since, and in modified form is known today as neo-Lamarckianism. The conception is simple enough and has a basis of facts. It is well known, for example, that use develops a muscle, and that disuse deteriorates it, a deterioration that may reach as far as inability to function. If this effect of use and disuse be applicable to all organs and regions of the body, and certain conditions of living were to change, demanding the use of structures that had not been called upon to do so much service before, and also excusing from such constant service structures that had been very active before, one might imagine changes taking place in the greater development of certain structures and the less development of others. In other words, change in the environment means change in the demands on the structures of plants and animals, and these demands are met by the active exertion of the organism.

A well-known illustration used by Lamarck will serve our purpose. A grazing animal, with an ordinary neck, is placed in conditions that demand feeding upon the foliage of trees. The continuous use of the neck in stretching would cause it to increase somewhat in length. This slight increase in length would be transmitted to the next 
generation, which in turn would add to it, until a number of generations would succeed in developing the exaggerated neck of the giraffe.

This illustration makes clear the factors relied upon by Lamarck, namely, the effect of use demanded by changed conditions, and the transmission of the changes from parent to offspring. His own name, "appetency," sought to express the idea of striving to satisfy a desire; but, as might have been expected, it was not understood by most of the people of his day, and lent itself admirably to all sorts of caricature.

The changes in structure brought about during the life of an individual are spoken of as "acquired characters," and Lamarck's explanation of the evolutionary process would be impossible if acquired characters are not transmitted from parent to offspring. The present consensus of opinion seems to be that such acquired characters as Lamarck had in mind are not transmissible; but the whole subject of the transmission of acquired characters is more a matter of definition than anything else.

3. Natural selection.-It was the explanation offered by Charles Darwin, however, that proved to be the most epoch-making theory in the history of biological science. He called it "natural selection," and it has been a dominating conception for fifty years. With the Darwin centennial celebrations only two years old, and with the flood of literature that accompanied and followed them, no one interested in the subject of evolution can be ignorant of the meaning of natural selection, or of the revolution in thought and method brought about by its presentation in Darwin's Origin of Species. While Lamarck's conception was based upon extensive observation, and therefore was 
reached in a thoroughly scientific way, Darwin's conception was based upon an amount and range of observation hitherto unapproached; so that if Lamarck's approach was scientific, Darwin's was still more scientific. In fact, Darwin's announcement came at a psychological moment, which enormously reinforced his message; and this is not detracting in the least from the power and beauty of its presentation. Whether Darwin's explanation stands or falls, his supreme contribution must be regarded as the introduction of a point of view and a method of attack that not only ushered in modern biology, but also revolutionized thought in general.

Natural selection is too familiar to need extended explanation. The ratio of increase of organisms, leading to over-production and a struggle for existence, resulting in the survival of the fittest, is a series of exceedingly familiar phrases, not all of which should be attributed to Darwin. That plants and animals can be led along in any desired direction was proved by experimental evidence obtained from the operations of plant and animal breeders; and since this guidance of plants and animals by man was by means of selection, it was most appropriate to call the guidance by nature "natural selection."

The most significant fact connected with this theory remains to be mentioned, and that is the fact of variation. Nothing is more clear than that any machinery of evolution must depend upon this fact. Darwin greatly enlarged the horizon of our knowledge in reference to variation. It is variation that gives rise to individuality among plants and animals, so that no two plants or animals are exactly alike. We have accustomed ourselves to individuality among human beings, for we have been trained to note the dis- 
tinguishing marks. But this same individuality is no less true for all animals and plants. 'In heredity, therefore, there is transmitted not only a likeness to the parent, but also an unlikeness, and this unlikeness constitutes individuality, a certain amount of variation from the parent.

Darwin's conception was that nature selects from among these varying individuals; that the means of selection is the competition that results from over-production; that the better adapted individuals would naturally be selected for survival; that their better adaptations, which mean their individual peculiarities, would be transmitted to their offspring; and that such selection, continued generation after generation, would so emphasize and increase the favored variations that the old species boundary would be crossed and a new species established. In other words, small variations would be built up into larger ones, and presently they would become too large to be included within the boundary of the old species.

Of course, objections have been raised to the theory of natural selection as an adequate explanation of the origin of species. There can be no doubt but that there is selection in nature, in the sense that not all the forms produced survive; but many believe that this cannot change forms enough to be regarded as new species; that any selection thus made cannot be on the basis of any "life-and-death" advantage of structure that one individual has over another; and that the variations thus used are only the so-called "fluctuating variations" which have nothing definite in them as to direction or amount.

4. Mutation.-We come now to the work of the last decade, which is characterized by the rapid development of the study of evolution by using experimental methods. 
Perhaps the most influential work to enforce the experimental method was that of DeVries, in developing his theory of mutation. His great contribution, therefore, must not be regarded as offering mutation as an explanation of the origin of species, for that explanation may not stand, but as establishing, or at least powerfully helping to establish, the study of evolution upon an experimental basis.

The mutation theory needs no extended explanation, for the current literature of organic evolution is full of it. The long series of cultures of Oenothera, under rigid control and in large numbers, are familiar. The appearance, in relatively very small numbers, of widely different individuals, which "came true" in subsequent generations, led to the inference that new species were appearing under observation, suddenly produced by the parent form, fully equipped as species, without any intermediate stages or any building up by selection. It should be noted that this does not banish natural selection as a factor in evolution, but assigns to it a new rôle, which is not to produce species, but to select among those already produced.

The study of mutations is one of the vigorous phases of experimental work today, and some of the results will be presented in the subsequent chapters. Objections to the theory have developed, as must be the case in all theories. There are questions as to the extent of mutation as a process going on among plants and animals; as to its reliability in producing species; as to whether mutants are really new forms, or only old ones derived from a splitting hybrid parent. It is such questions, and others like them, that experimental work today is trying to answer.

5. Orthogenesis. - The barest kind of evolutionary background would be inadequate without a mention of ortho- 
genesis. The variations utilized in the preceding explanations, both the smaller ones used by natural selection and the larger ones used by mutation, occur in every direction from the parent form, the successful direction being determined by natural selection. This has been called indeterminate variation. In tracing the evolution of great groups, however, it becomes clear that the most important variations occur in certain definite directions, which have been maintained persistently throughout all possible changes of condition. For example, the history of such a group as gymnosperms shows a tendency to vary in certain definite directions that has persisted from the early Paleozoic to the present time. In other words, there is much to indicate that while variation may be indeterminate, there are also certain definite lines that persist. The origin of new forms, whether by natural selection or mutation or neither, as the result of a persistent determinate variation, is called orthogenesis. It certainly removes one of the greatest difficulties in the way of natural selection, and that is the beginning and development of a structure that can be of advantage only when it is completed. It satisfies also the many known cases of excessive development in certain directions, a development that may be not only disadvantageous, but even destructive. Even if determinate variation is accepted as a fact, however, what determines the persistent variation? The answer to this question has resulted in many rariations of the theory of orthogenesis.

It should be noted that natural selection, mutation, and orthogenesis are not mutually destructive. They all deal with variations, and may all be operative in producing new forms. Natural selection deals with small rariations which are in every direction; mutation with large variations which 
are in every direction; and orthogenesis with those small or large and relatively few variations which for some reason persist and increase from generation to generation and carry forward the group as a whole.

\section{BIOMETRY}

When the idea of natural selection became dominant, and new species were believed to have arisen by the accumulation of small variations, which seemed to be indefinite and fluctuating, a statistical method of attack began to be developed, a method that has been named biometry. The most conspicuous names that one meets in the literature of biometry are Galton, the English pioneer in the exploitation of the method; and Pearson, who has been largely instrumental in carrying it forward into its more advanced mathematical stage.

It is a method that deals with groups or populations, rather than with individuals, and its results present the averages of variations. It is evident that an average obtained from the measurements of a given character in a series of individuals will depend upon the individuals selected for measurement. Therefore, biometry demands to an unusual degree the elimination of preconceived opinions and the exercise of great judgment. It has become so special and intricate a method that it can be followed, with full understanding, only by those with special training; so that any adequate illustration of it will be left to such of the subsequent addresses as may have occasion to apply it.

A word can be said, however, concerning its use as an instrument in the study of the processes of evolution. Selecting any character or group of characters that are to be measured or counted, and using a wisely selected range of 
material, biometry reveals the prevailing tendency, in reference to these variations, in a group of individuals representing a species, a tendency that could not be recognized by the study of a single individual. Applying this method to successive generations from this group of individuals, under experimental control, it can be discorered whether the prevailing tendency in the expression of these variations remains the same, continuing the species as before; or shifts, modifying the species as a whole; or splits up, giving rise to a second marked tendency, that may mean presently two distinct species.

It is evident that such data can be very suggestive, but that their limitations must be recognized. They are data concerning successive populations, and show the average result of individual variation as expressed in a population. In other words, they present in concrete and somewhat definite form the problems of variation and inheritance that must be solved.

\section{HEREDITY}

It must have become evident, during the preceding sketch of representative theories of evolution, that the fundamental factor in the process is variation, and that the essential and inevitable question behind all of these explanations is the origin of variation. This brings us at once to the problem of heredity, with its supposed processes for transmitting what we call "characters" from parent to offspring. How is variation secured in this transmission? The earlier observers simply accepted variation as a fact, and made no serious attempt to explain it.

The first attack upon this problem was the accumulation of data in reference to the facts of heredity. To accumulate 
these facts in such numbers as to make any generalization worthy, demands the culture through many generations, under most rigid control, of the largest possible number of plants and animals. This means long periods of time, great patience, and many investigators. The number of investigators is multiplying, the range of material is increasing, and the period covered by some of the work has now been sufficient to justify some presentation of the results.

Probably the most conspicuous working hypothesis today, in connection with the collection and interpretation of the data of heredity, is the one called "Mendel's law." This is to be made a special theme in the course of this series, but a brief statement in reference to it will help the background and may prepare the way for the later discussion. This Austrian monk, who worked in his monastery garden during the middle of the last century, left on record what is called a law of heredity. This record was lost, so far as its influence was concerned, until ten or fifteen years ago, when the modern movement in experimental evolution began to be vigorous. Now the Mendelians constitute a conspicuous biological cult, and Mendelism has extended from its simple original statement into a speculative philosophy, with conceptions of unit-characters, dominance, ratios, etc., that the untrained cannot follow.

The fundamental conception is simple enough. If two different species are crossed, the result is a hybrid which combines certain characters of both parents. When this hybrid propagates, the progeny splits up into three sets: one set resembling the hybrid parent; and the two other sets resembling the parent forms that entered into the hybrid. Mendel's law is a statement of the definite ratio 
expressed by these three groups of forms derived from a splitting hybrid. This means that in a series of generations initiated by a hybrid, approximately one-half of the individuals of each generation will represent the hybrid mixture, one-fourth of the individuals will represent one of the pure forms that entered into the hybrid, and the remaining fourth will represent the other pure form.

It should be understood that the use of hybrids in such experimental work is simply a device to secure easy recognition of the contributions of each parent to the progeny. For example, if red and yellow races of corn are crossed, it is very simple to recognize the color contribution of each parent to the hybrid progeny, when it would be impossible to separate the contribution of two yellow parents. The inference is, that what is true of hybrids is true of forms produced in the ordinary way, so that laws of heredity obtained from a study of hybrids may be regarded as laws of heredity in general. In one sense, every union of parent forms is hybridizing, for each parent has its own individuality.

One of the more subtle problems that has arisen in connection with such investigations is the problem of sex determination. In all organisms with sex differentiation, progeny is produced by the fusion of male and female sexual cells, and this progeny develops as distinct male and female individuals. It is one thing to determine the general structure of an organism by some law of heredity, but a very different thing to determine why any individual thus produced is sometimes male and sometimes female.

The work of today is not resting content with the patient collection of the facts of heredity, with determining ratios as expressions of laws, and with the end results of processes 
initiated under experimental control. There is keen searching for cytological evidence; and the structure and behavior of the sexual cells, through the whole process of their formation, during the act of fusion, and in the initiating activity of the fertilized egg, are being subjected to all the scrutiny that a developing technique can devise to discover the mechanism of heredity. Moreover, cytological evidence is being searched for not only to discover a possible physical basis for heredity, which would mean actual material machinery, but also to discover the possible relations of chemical and physical factors to this most fundamental and obscure process. It is believed that it must be a response, in terms of chemistry and physics, by a living material substance.

\section{PRACTICAL APPLICATIONS}

In a university atmosphere, the chief interest is probably focused upon the attempt to reveal one of the so-called "mysteries" of life, those mysteries which always invite one to uncover them; but there is another aspect of these problems worth considering. The experimental work that has been done in the study of heredity and evolution has had a very important bearing upon the practical handling of plants and animals, including the human animal. The applications have been made to plants most extensively, and methods of plant breeding have been revolutionized. The recognition that commercially "pure seed" is an extensive mixture of different types or strains, has led to their separation, and has changed the clumsy and inefficient method of mass culture to the definite and exact method of pedigree culture. As a consequence, the number of forms made available for culture has been multiplied enormously, 
having simply been discovered and pedigreed, without the labor of continuous selection year after year, and without the old inconstancy in the result. To reduce labor, to multiply cultural forms, and to obtain constant results in plant breeding are results of very large importance to the material side of human welfare. Pedigree culture has not only multiplied available forms, but it has begun to be used most effectively in combating drought and disease, the most dangerous enemies of cultivated plants. Droughtresistant races are being developed from pedigreed stock, and immunity to different diseases has been found to be a transmissible character. When it is remembered that drought-resistance not only insures crops over areas now cultivated, but also secures an enormous extension of area that can be cultivated; and that the annual losses from plant diseases represent an enormous financial total; it will be appreciated that the study of heredity and evolution, with purely scientific purpose, incidentally has been enormously profitable on the practical side.

I have sketched a background that will permit those who follow to put their work in its setting without much loss of time. The interest lies chiefly in the foreground that they will develop, for it will represent the work of today. 


\section{CHAPTER II}

THE PHYSICAL BASIS OF HEREDITY AND EVOLUTION FROM THE CYTOLOGICAL STANDPOINT

Heredity involves not only the transmission of similarity in structure, but also the transmission of dissimilarity. Likeness means close relation to the parent forms; unlikeness means individuality. It is not generally appreciated that individuality expresses itself just as certainly among plants and animals as among human beings. We have learned to recognize the marks of individuality among human beings through long acquaintance, but we should realize also that no two plants or animals are exactly alike. It is this individuality that is called variation, and variation is the basis of evolution. The phenomena of heredity established as facts by series of cultures under rigid control, however, must be recognized as the end results, between which and the act of fertilization there extends a series of unknown processes, with which as yet only scientific imagination deals.

The purpose of this chapter is to inquire whether there is any physical basis for the transmission of like and unlike characters, in the same sense that protoplasm was long ago called "the physical basis of life." This phrase only means that protoplasm is the material substance in which the phenomena of life are manifested. Is there any substance or structure by means of which the phenomena of heredity manifest themselves?

The answer to this question would be given more appropriately by some biologist who has made it the special 
subject of his investigations. This chapter, therefore, must be regarded simply as the presentation of a teacher, to explain a subject that belongs logically in the series. Another restriction is that this presentation deals chiefly with structures that are visible by means of laboratory technique, and not with the results of experiment upon these structures. A final restriction is that the statements deal with plants, a limitation necessary to the writer, and offset by the fact that the corresponding facts in animals will be stated in one of the later chapters.

Some conception of what is meant by the power of reproduction will be useful. Among the simplest plants, every cell has this power; in fact, some plants are so simple that the adult body consists of a single cell. As plant bodies came to be made up of numerous cells, some of them lost the power of reproduction; and as the body became increasingly complex, the number of cells retaining the power of reproduction became relatively smaller. This means that in the complex plant body, the relatively few reproductive cells are not so much "cells set apart for this special function," as cells that have not lost this primary power. The specialized cells are not those that reproduce, but those that cannot. The loss of reproductive power is usually not complete, for most cells can reproduce their own kind, even if they cannot reproduce the whole body.

This leads to a consideration of what is included in full reproductive power. Without including confusing details, it may be said that such reproduction as one has in mind in connection with heredity involves four general things. First, there is cell multiplication, the fertilized egg initiating a series of cell divisions that may result in a multitude of cells. It is evident, however, that a complex plant body is 
more than a multitude of similar cells. In the second place, there is cell differentiation, groups of cells becoming different, so that the various tissues, with their special functions, are developed. In the third place, tissues must be organized together into the structures called organs. In the last place, the organs must be combined in making that total organization called the individual. It is this far-reaching directive influence that is the most baffling fact in connection with heredity.

To obtain any impression of the supposed machinery of heredity, it is necessary to know something of the structure of a living cell. So far as material goes, such a cell is an individualized mass of protoplasm. This protoplasm is organized into a body known conveniently as the protoplast, which is the living body of the cell. In plants, the protoplast usually constructs a cellulose wall about itself, which has given rise to the impression that a cell is a walled chamber containing protoplasm. 'In the plant cells which have to do directly with heredity, however, namely the reproductive cells, the cellulose wall is not formed, and they are naked protoplasts. The protoplast is exceedingly complex, as cytologists well know, and includes a variety of organs. Conspicuous among these protoplasmic organs is the mucleus, which is a more or less spherical body and usually sharply limited from the rest of the protoplast, in which it lies imbedded (Fig. I). The remainder of the protoplasmic material enters into the structure of the cytoplasm, another organ or region of the protoplast constantly associated with the nucleus. Every living cell contains a nucleus and cytoplasm, and in addition there may be other protoplasmic organs (Fig. I), but the two mentioned are those that belong to this discussion. 
As in the case of all organs, the cytoplasm and the nucleus are associated with special functions. This does not mean that each does a certain thing and nothing else, but that each is conspicuous in comnection with a certain kind of work. Especially unsafe is it to ascribe certain definite functions to these organs of the protoplast, because protoplasm itself is so little understood. In any event, the cytoplasm seems to be conspicuously associated with the metabolic activities of the cell; and it is certain that the nucleus is conspicuously associated with cell division. When division occurs, and one cell gives rise to two cells, this process almost invariably begins with the nucleus, which may thus be said to initiate cell division. It must be understood clearly that we are speaking of visible changes in structure, behind which and

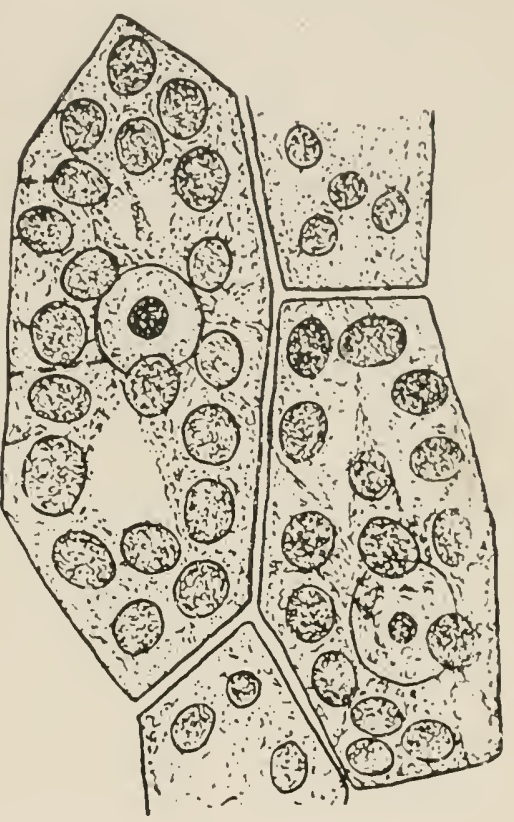

Fig. I.-Cells from a moss leaf: each of the complete cells shows the welldefined nucleus; since the leaf is green, there are also numerous green protoplasmic organs (chloroplasts); the remaining granular-looking ground substance is the cytoplasm. accompanying which there are certainly numerous invisible physical and chemical changes.

It is evident that the problem of heredity is involved in the process of cell division, for through this process the old cell transmits whatever determines the characters of 
the two new cells. In ordinary cell division, the immediate transmission seems to be a similar structure, for the new cells resemble the old one in all recognizable features. In the differentiation of cells, however, certain cell divisions involve the transmission of unlike characters.

This relation of the nucleus to cell division, and of cell division to heredity, has focused the attention of cytologists upon the structure and behavior of the nucleus. No structure of plants and animals has received such detailed and persistent investigation as has the nucleus, and much of the advance in technique associated with the use of the microscope has been stimulated by the necessity of learning more about the nucleus.

If the nucleus is the conspicuous structure associated with cell division, the suggestion is natural that it is the material structure associated with heredity. But the nucleus is a complex, and most conspicuous in its structure is a substance called chromatin. In the ordinary nucleus it appears as a network of denser material, which has received its name from the fact that it takes stains easily, being the most stainable substance in the nucleus (Fig. $2, a)$. If there is any definite material that deserves to be called the physical basis of heredity, it is probably chromatin, which of course is a protoplasmic substance. This belief is largely based upon the behavior of chromatin during cell division.

In preparation for division, the chromatin network becomes an evident continuous band, which resembles a tangled skein (Fig. 2, b). This band shortens and correspondingly thickens, and finally breaks up into a definite number of units, called chromosomes (Fig. 2, c). These chromosomes are thus chromatin individually organized, 
in the same sense that protoplasts are protoplasm individually organized, and they are thought to retain their individual identity through all the apparent fusions into bands and network. It is the chromosome, therefore, consisting of the material chromatin, that is regarded as the organized carrier of transmissible characters; hence the behavior of the chromosomes in cell division becomes a

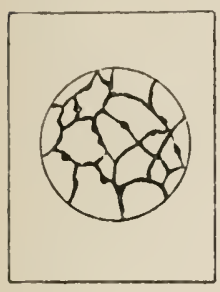

$a$

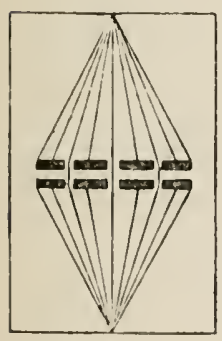

$\ell$

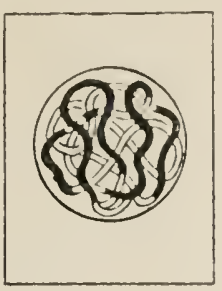

$b$

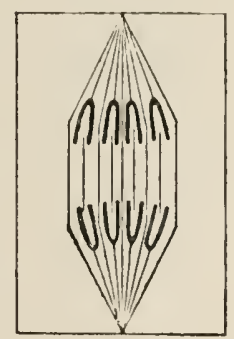

$f$

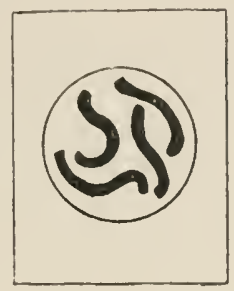

C

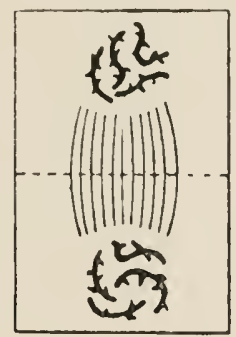

$g$

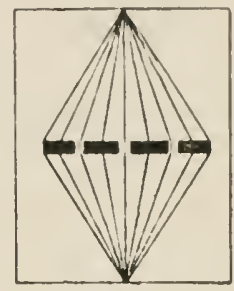

$d$

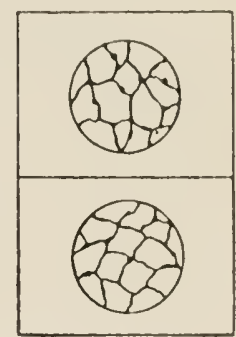

$h$

FiG. 2.-Diagram of stages in nuclear division.-After Lock

matter of first importance in considering the machinery of heredity.

One of the important facts to note is the definite number of chromosomes. Each kind of plant and animal has its own number. For example, in certain plants the number is 6 ; in others it may be considerably more than Ioo; and of course the intermediate numbers are well represented. Differences in the number of chromosomes may occur 
among very closely related plants, or the number may be constant throughout a great group. For example, in the gymnosperms, so far as known, the number is almost constantly I2. This fact has given rise to the suggestion that the number of chromosomes, as well as their quality, may be a factor in heredity, but too much stress must not be laid upon it as yet.

While the chromosomes are becoming separate, a spindle of fibers is formed about the nucleus, and the chromosomes become attached to the fibers, finally being arranged about the equator of the spindle (Fig. 2, d). In this position, each chromosome splits longitudinally (Fig. 2, e), and the two halves, by the shortening of attached fibers, are drawn toward the opposite poles of the spindle (Fig. 2, $f$ ), the old chromosome thus being represented at each pole by a half-chromosome. The half-chromosomes at each pole enter into the organization of a new nucleus (Fig. 2, g), wall material is deposited in the plane of the equator of the spindle and, extending through the cytoplasm, cuts the old cell into two new cells, each with its nucleus (Fig. 2, $h$ ). It is evident that each new nucleus has the same number of chromosomes as the old one, and that each chromosome of the new nuclei represents in material a chromosome of the parent nucleus.

This detailed and precise process in the behavior of chromosomes, insuring the transmission from one cell to its progeny cells of the identical material contained in every chromosome, is a strong argument in favor of regarding the chromosome as the carrier of hereditary qualities.

The kind of reproduction with which the problems of heredity are concerned chiefly, however, is that which involves the fusion of sexual cells. There are three distinct 
methods of reproduction recognized among plants. The most primitive method is vegetative multiplication, ordinary vegetative cells producing new plants. In the transmission of hereditary qualities this involves simply a series of such cell divisions as has been described above.

Later in the evolution of plants, the power of reproduction was displayed chiefly by spores, which at first were only certain protoplasts that escaped from the incasing wall. In most cases, before escape the protoplast divides, so that there issue from the old cell two or more naked protoplasts or spores. Since the early spores belonged to water plants, they had swimming appendages (cilia), and were called swimming spores or zoöspores (Fig. $3, a$ and $b$ ). Any one of these swimming spores, under appropriate conditions, can produce a new individual. This method of multiplying individuals remains the most effective method among plants.

Vegetable multiplication and

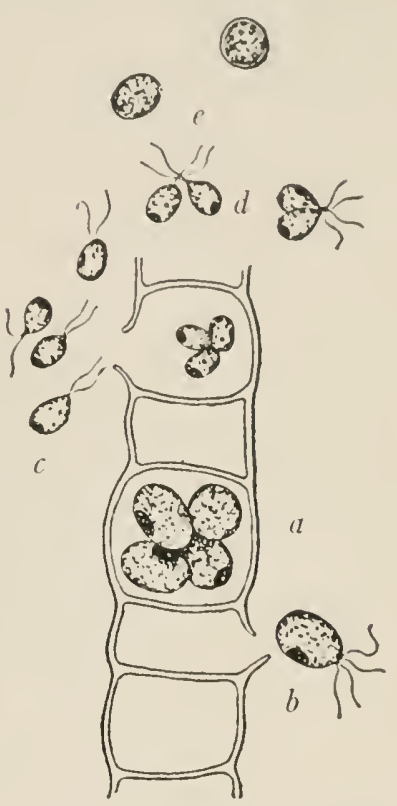

Fig. 3.-A portion of a filament of a green alga (Ulothrix): $a$, zoöspores in mother cell; $b$, an escaped zoöspore; c, gametes, most of which have escaped from the mother cell; $d$, gametes pairing and fusing; $e$, zygotes. reproduction by spores are both sexless methods, and it is quite evident that the introduction of the sexual method is more significant than merely a third method of reproduction.

It is demonstrable among plants that the sexual cells (gametes) were derived from the swimming spores (zoöspores). In certain plants, if a protoplast divides and gives rise to 
few and comparatively large protoplasts, they are swimming spores (Fig. 3, $a$ and $b$ ), and each can reproduce. If the divisions continue, however, and result in numerous and comparatively small protoplasts, they are unable to reproduce (Fig. 3, c). However, if they come together in pairs and fuse (Fig. 3, d), thus making one cell (protoplast) out of two (Fig. 3, e), the new cell can reproduce. This fusion is the sexual act, and the fusing cells are the sexual cells (gametes). It is of interest to note that this first appearance of sex is quite disconnected with the multiplication of individuals. Individuals are multiplied throughout the growing season by the spores. Toward the close of the season, the gametes begin to appear, and the fusion cells (zygotes) formed by their pairing develop heary walls that protect them through the unfavorable season (as the winter). All the other structures of the plant perish, and it exists through the winter only in the form of zygotes. At the beginning of the next season, the zygotes produce new plants, and these are multiplied by spores. The service rendered by the sex act in this case, therefore, is to produce a protected cell, which can carry the plant through an unfavorable period; in short, the service is protection rather than multiplication.

The next advance in the evolution of sex was its differentiation. The gametes at first are similar in appearance and in behavior, but it must be recognized that this optical test would be unable to detect any differences in quality. It is upon the basis of appearance that such gametes are said to be unisexual, which only means that they cannot be distinguished as male and female. A series can be arranged to illustrate a gradual differentiation of gametes into two forms, unlike in appearance and in behavior. In 
one case, the gamete becomes larger and larger, its power of movement diminishing at the same time, until at last it becomes a very large and entirely passive cell. In the other case, the gamete retains its small size and activity. These two very dissimilar gametes are the egg and the sperm, easily distinguishable female and male cells (Fig. 4). The essential difference thus brought about is the great in-

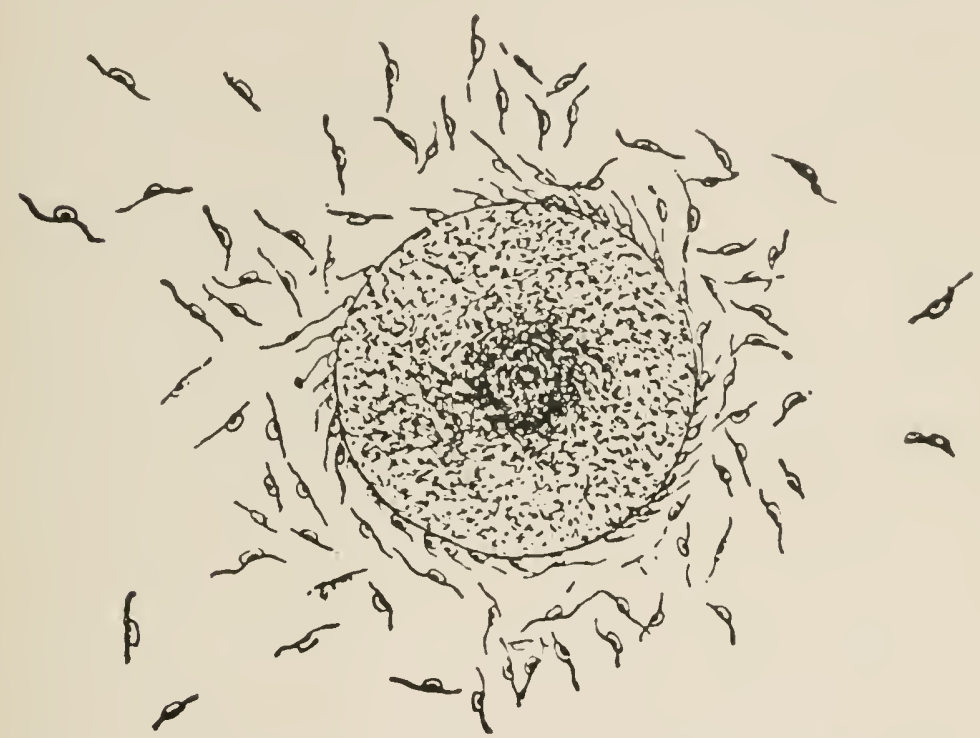

FIG. 4.- I single large egg and numerous small sperms of rockweed (Fucus)

crease in the bulk of the gamete that becomes the egg, but the constant feature, which is not changed, is the chromatin, the increase in bulk being due to an increase of cytoplasm. This constancy of the chromatin, coupled with the known fact that the two gametes contribute alike to their progeny, indicates that the chromatin is the essential material in heredity. Since both cytoplasm and nuclei are involved in the sexual fusion, it may be claimed that the 
cytoplasm is as essential to the process as the nucleus; but in certain plants, notably Lilium, it has been demonstrated that when fusion occurs there is no cytoplasm whatsoever investing the male nucleus. It seems safe to conclude, therefore, that the nucleus contains the material essential to the phenomena of heredity; and if so, chromatin must be the material, and the chromosomes its visible organized units.

A very simple case will serve to illustrate the results of the sexual fusion upon the chromosome situation. Imagine

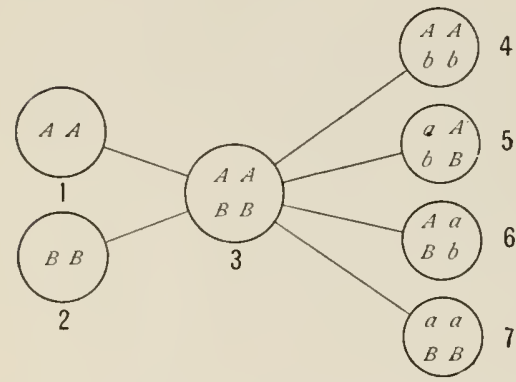

Fig. 5.-Diagram illustrating result of sexual fusion: 1 , sperm containing two chromosomes $(A A) ; 2$, egg containing two chromosomes $(B B)$; 3 , fertilized egg containing four chromosomes (two paternal and two maternal); 4 , domination of paternal chromosomes; 5 and 6 , domination of one paternal and one maternal chromosome; 7 , domination of maternal chromosomes. the fusion of an egg and a sperm, each of whose nuclei contains two chromosomes (Fig. 5, I and 2). The nucleus of the fertilized egg would contain four chromosomes (Fig. 5, 3), two of them maternal (contributed by the egg), and two of them paternal (contributed by the sperm). Suppose that two of the four dominate in determining the structure of the new individual to be developed from the fertilized egg. It will be seen that there are four possible pairs: (I) the paternal pair (Fig. 5, 4), in which case the new individuals would resemble the male parent; (2) the maternal pair (Fig. 5, 7), in which case the resemblance would be to the female parent; (3) two pairs (Fig. 5, 5 and 6 ), each consisting of a dominant male and a dominant 
female chromosome, in which case the new individual would be a mixture, resembling both parents. Expressing the chances in the form of a ratio, they could be represented as $\mathrm{r}: 2: \mathrm{r}$. This is a simple expression of Mendel's law, defined in the preceding chapter. The formulation of Mendel's law was based upon the observed facts of heredity; and this chromosome situation supplies for it a cytological basis.

It must be understood clearly that so simple an illustration does not represent the actual facts, for chromosomes are usually more numerous, and eggs and sperms already contain a mixture of paternal and maternal chromosomes derived from the preceding generations. It does illustrate, however, the mixture of hereditary qualities by the sexual fusion, the domination of certain of these qualities, and the chances of resemblances in the progeny. It should be understood also that the structural resemblance to the maternal form or to the paternal form does not include sex determination. For example, the new individual which resembles the maternal form may prove to be either male or female, and vice versa.

It is evidently impossible that the chromosomes continue to be doubled at each generation, without any reverse process of reduction. The two cardinal points in every lifehistory, therefore, are fertilization, by means of which the chromosomes are doubled, and reduction, by means of which the doubled number is halved. The reduction process occurs at different stages in the life-history in different organisms. Among animals, it occurs in connection with the formation of the eggs and sperms; and therefore reduction and fertilization are practically consecutive events. In most plants, however, the two processes are farther apart, separated from one another by two distinct indi- 
viduals, one characterized by the reduced number of chromosomes and bearing the sex organs (hence called the gametophyte), the other characterized by the doubled number of chromosomes and producing spores (hence called the sporophyte). In general, it may be said that among animals reduction occurs in connection with gamete forma-

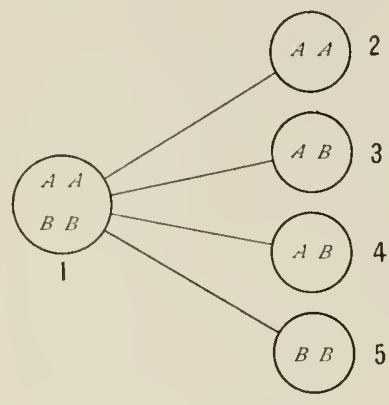

FIG. 6.-Diagram illustrating result of reduction: $I$, ordinary cell of the body containing doubled number of chromosomes; 2-5, eggs or sperms containing the reduced number, and illustrating th? possibilities in the distribution of paternal $(A A)$ and maternal $(B B)$ chromosomes. tion, while among plants it occurs in connection with spore formation.

To use the simpler illustration of animals, it is evident that the cells making up the body of an individual contain the doubled number of chromosomes (Fig. 6, I), derived from the fertilized egg from which the body developed. When the sexual cells (eggs or sperms) of this individual are formed, the reduction occurs, and the eggs or sperms contain the reduced number. How the maternal and paternal chromosomes are distributed in this reduction may not be clear, but it is evident that either eggs or sperms may contain chromosomes derived from either line of descent (Fig. 6). In other words, it is not inconceivable to think of an egg containing only chromosomes derived from the paternal side, or of a sperm containing only chromosomes derived from the maternal side, or most likely of both eggs and sperms containing chromosomes derived from both sides (Fig. 6, 2-5).

These considerations indicate how every sexual fusion results in a complex of possibilities, and the study of heredity 
is to discover whether these possibilities can be formulated into a law.

In this connection, the phenomenon of parthenogenesis should be referred to. By definition, it means the production of a new individual by an unfertilized egg, and it is a very common phenomenon among plants. When it is remembered that ordinary cells can produce new individuals by regetative multiplication, and that spores can reproduce, it should not be thought strange that so wellnourished a cell as the egg can do the same thing. Among the higher plants, however, in which the whole mechanism has been worked out in greater detail, there is a significant fact connected with the cases of parthenogenesis. In every case investigated, the reduction in connection with spore formation has not occurred, and therefore the unfertilized egg contains the doubled number of chromosomes, just as though it had been fertilized. In parthenogenesis, therefore, the indications are that the fact to be explained is not reproduction by an unfertilized egg, but the failure of reduction.

The whole history of sexual reproduction among plants indicates that its primary significance is not reproduction, for probably many more individuals are produced by regetative multiplication and by spores than by the sex act. This would mean that the sexual method is chiefly concerned with other results, which are secured in connection with reproduction. These results seem to be the continual securing of new combinations, and new combinations certainly make for evolutionary progress. 



\section{WILLIAM ERNEST CASTLE}

Professor of Zoölogy, Harvard University 



\section{CHAPTER III}

\section{THE METHOD OF EVOLUTION'}

No one today doubts the reality of evolution, at least no one does who has had practical experience in animal or plant breeding, and who has seen new forms of life come into being under his own observation and guidance. But the method of evolution is still in doubt. It is known in general, that like begets like, but that occasionally it begets unlike, and this may become a new race. As to how the new race is begotten we have not got much beyond Darwin; indeed many of us have not got so far. For Darwin recognized two distinct ways in which new races may arise, but many biologists today insist that there is only one way, the way in which Minerva was begotten, who "sprang full-fledged from the head of Jove." The modern name for this method of origin is mutation, and its advocates, like the "followers of the prophet," insist that there is no other.

Darwin was well aware that new races may arise in this way, particularly under domestication, as in the case of the Ancon ram and Niata cattle; but he believed that a far commoner and more important method, particularly among wild species, consists in a slow and gradual modification of the race, constantly in one direction, as under the ever-growing power of a hydraulic press, until the

I In these two chapters, especially in the second (chap. iv), material has been drawn freely from the writer's book on IIeredity in Relation to Evolution and A nimal Brecding (D. Appleton \& Co., New York), for which he has the kind permission of the publishers. He also wishes to acknowledge aid given by the Carnegie Institution to the investigations herein described. 
descendants become so different from their progenitors that man assigns them to distinct races.

Now I am inclined to think that Darwin was on the whole nearer the truth than the mutationists. They have perceived a half-truth and perceived it more clearly than did Darwin, but in scrutinizing this they have lost sight of the larger picture which he saw. Darwin saw that new races arise in two ways, and I shall attempt to show that he was right.

First let us discuss the Minerva-like method of evolution, the birth of new races in a day, a method of great theoretical interest and practical importance. What is known about this method of evolution is commonly called Mendelism, after Gregor Mendel, an Austrian monk of the last century. Mendel was a school teacher who studied as well as taught, and fortunately for us he studied from the book of nature more than from other books. He thought clearly about the things he saw, but wrote little. Indeed we wish that he had written more, but perhaps if he had done so he would have thought less well. Like most profound thinkers, he was in advance of his day, so that when he spoke, the "wise men" of the time failed to understand him. The "wise man" to whom Mendel hoped to make his ideas plain was the great German botanist, Karl Naegeli, to whom Mendel wrote a number of letters about his studies of plant hybrids. Naegeli failed to grasp the important point in Mendel's work, and the letters were forgotten until Mendel's fame had become world-wide. Then they were hunted up and published. Naegeli's failure to understand Mendel is after all not surprising; Mendel's thinking was in advance of his time. Several biological principles now considered commonplaces were then un- 
known. When these had been established, Mendel's law was independently rediscovered long after his death. All honor to the rediscoverers, DeVries, Correns, and Tschermak, that, honoring the all-but-forgotten monk, they called the new-found law Mendel's, rather than their own!

In Mendel's time little was known about the nature of the reproductive bodies from which new individuals arise, or of how these bodies are produced, or how they differ from the organisms which produce them. These points must be considered briefly.

An old but ever-recurring question in regard to heredity is this: Does one generation inherit any part of the experience of the previous generation? In other words, is a character acquired by one generation inherited by the next? This question, first raised in concrete form by Weismann, has been discussed pro and con for many years, but the consensus of scientific opinion at the present time favors Weismann's idea that acquired characters are not inherited. In forming a judgment on this question, one fundamental fact should be borne in mind, that in the higher animals body plasm and germ plasm are distinct; that is, the body is distinct from the reproductive cells which it contains, and out of which the next generation is produced. Influences which affect the body have no necessary influence on the germ cells.

Weismann some years ago demonstrated this experimentally for mutilations of the body. When the tails of mice were cut off generation after generation, it was found that young of the mutilated parents had tails as long as other mice. More distinct evidence of the independence of germ plasm and body is furnished by an experiment recently performed by Dr. Phillips and myself. 
A young female albino guinea-pig approaching sexual maturity was deprived of her ovaries, and into her body was introduced the living ovary of a freshly killed black guinea-pig, about three weeks old (Figs. 7 and 8). She was later mated with an albino guinea-pig (Fig. 9). By him she bore two litters of living young, and died pregnant a little over one year after the operation, containing a third litter (Figs. IO-I5). Had she not been operated upon, her young by this male would undoubtedly have been albinos, for albino guinea-pigs produce only albino young, as several investigators have clearly shown. But those young which she did bear were without exception black, which character clearly they owed to the fact that they developed from eggs produced by the ovary taken at a very immature stage from a black animal. From evidence such as this it is concluded that the inheritance can not be affected by modifications of the body of the parent, not even when the body is completely changed, since the body, so far as heredity is concerned, is merely a container of the reproductive cells. To modify the inheritance we must modify the reproductive cells.

But the reproductive cells are not simple; they are really dual in character, made up of equivalent parts derived from father and mother. On this matter breeding experiments throw light.

If a black guinea-pig of pure race is mated with an albino, the offspring are all black, yet contain albinism as a latent or recessive character. For if one of these black offspring is now mated with the same albino, only half of the offspring are black, the others being albinos. And if two of the cross-bred blacks are mated with each other, one-fourth of the young, on the average, are albinos, three- 


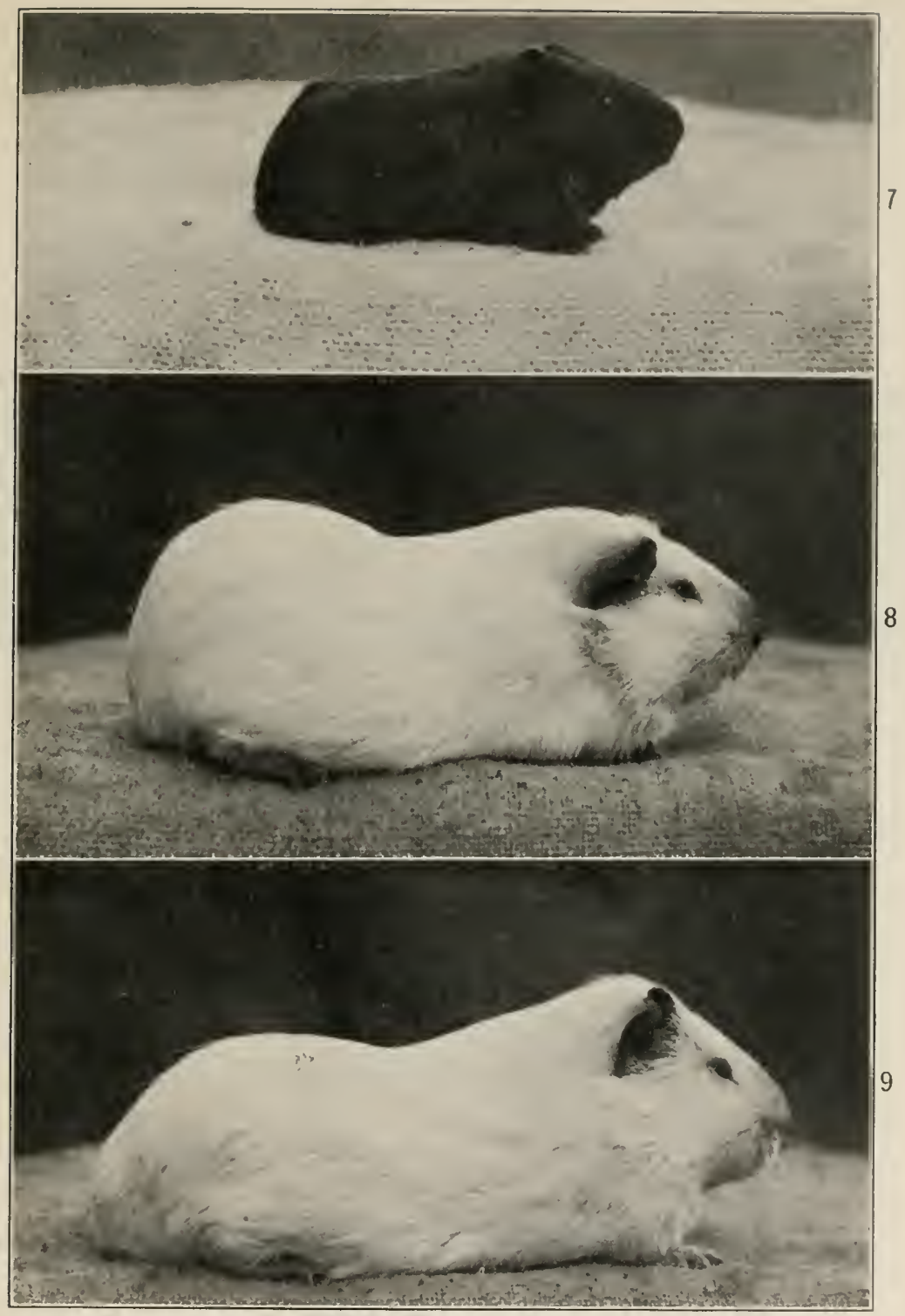

FIG. 7- $-A$ young, black guinea-pig, about three weeks old. Oraries taken from an animal like this were transplanted into the albino shown immediately below it.

Fig, 8.- An albino female guinea-pig. Its ovaries were removed and in their place were introduced ovaries from a black guinea-pig, like the one shown in Fig. 7 .

FIG. 9.-An albino male guinea-pig, with which was mated the albino shown in Fig. 8. 


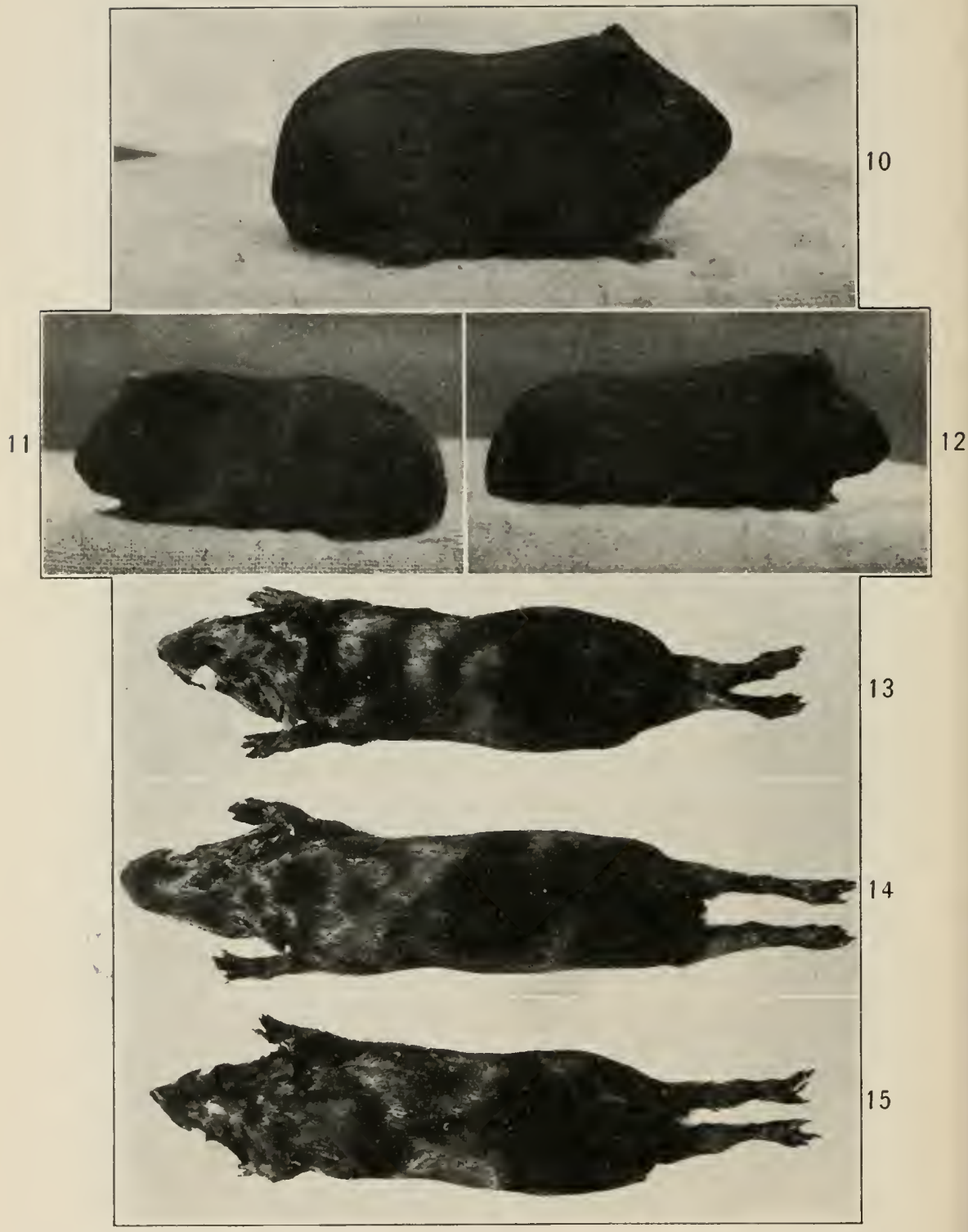

FIGs. 10-15.-Pictures of three living guinea-pigs (10-I2) and of the preserved skins of three others (I3-I5), all of which were produced by the pair of albinos shown in Figs. 8 and 9 . 
fourths being black (Figs. 16-19). This result is explained in the following way. The cross-bred black individual received from its black parent the character black (B), and from its white parent the character white (II). In it accordingly black and white were associated together, but only the former was manifested, the white remaining hidden by the black. But in reproduction the cross-bred black individual transmits black and white in separate cells. And since these two kinds of cells are in the long run equally numerous, it follows that the cross-bred black individuals produce both black and white offspring in proportions fairly constant (Fig. 20).

Inheritance of this sort is called Mendelian, after Gregor Mendel who first observed and explained it. The law governing such inheritance is called Mendel's law. Such inheritance is satisfactorily accounted for by the assumption that the reproductive cells are at first dual in nature, but become simple before they can function in the production of a new individual. For this assumption we have abundant evidence furnished by the direct study of the reproductive cells with the microscope. These cells, like the cells of the body in which they are contained, show in their nuclei at cell division a fairly constant number of bodies known as chromosomes. In the worm Ascaris there are only 2 of these chromosomes; in the sea-urchin Toxopneustes there are 36 ; in mice and men, about 24.

A new individual arises, in sexually produced animals, out of the union of an egg with a sperm. The sperm is relatively small, but its influence equals that of the much larger egg, which fact throws light on the nature of the material basis of heredity. It suggests, namely, that this material consists largely of ferment-like bodies which 
46

Heredity and Eugenics

$\simeq \quad$ o

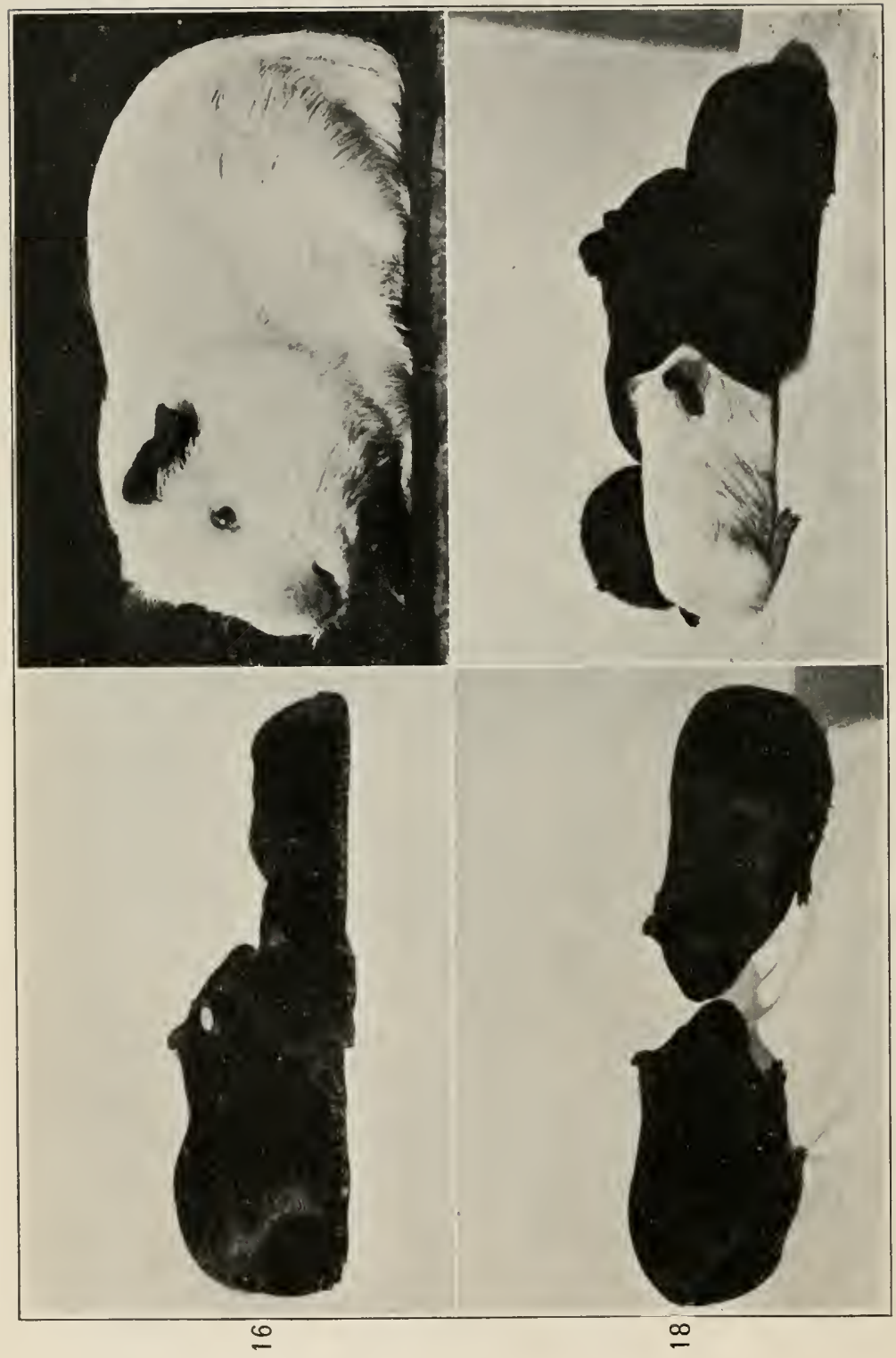

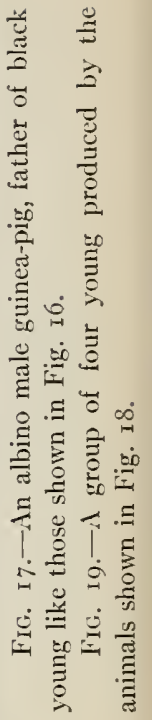

胥苛

를

总 플

范

ㄷำ

言

.

bo है

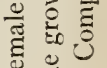

可

类客

ב

广

$0_{i=1}^{\infty} \stackrel{0}{-1}$

ن

I 
initiate specific metabolic processes in a suitable medium represented by the larger portion of the egg.

In egg and sperm, before their union, the chromosome number is reduced one-half, from the double to the single condition; in Ascaris, from 2 to I; in the sea-urchin, from 36 to $\mathrm{I} S$; in the mouse and in the man from 24 to 12. These reductions occur in what is called the maturation of the sexual products.

In the male, the primitive germ cell containing the double or $2 \lambda^{\top}$ number of chromosomes divides up into a group of four cells, each containing the single or $N$ number of chromosomes. This comes about by a failure of the chromosomes to split at one of the two cell divisions which produce the group of four sperm cells, as they regularly do in ordinary cell division. A

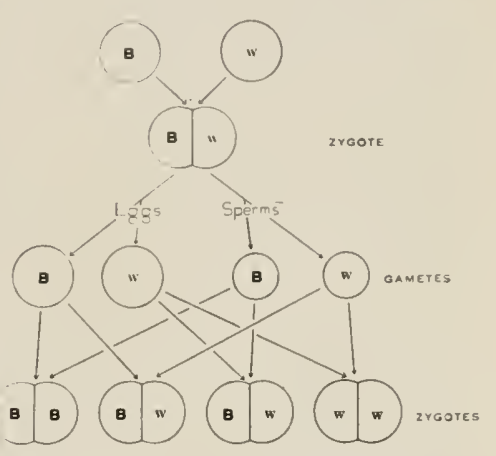

Fig. 20.-Diagram to explain the result shown in Figs. 16-I9. tadpole-like sperm now arises from each of the cells containing the reduced number of chromosomes.

In the maturation of the egg, reduction is likewise accomplished by two cell divisions, in one of which the chromosomes do not split as in ordinary cell divisions. The divisions of the egg, however, are into parts of very unequal size, only one of which is fertilized, the rest failing to develop. For example, in the marine worm Nereis, according to Wilson, the maturation of the egg occurs simultaneously with its fertilization. The first maturation division separates off a minute cell, known as the first polar 
body, and is quickly followed by a second likewise unequal division, by which a second polar body is produced. The number of chromosomes remaining in the egg nucleus is now reduced to half that in the egg before maturation. A sperm entering the egg has formed within it a second nuclear body which, like that of the egg contains a reduced number of chromosomes. By the union of these two nuclei a new nucleus is formed which contains the double number, and from this all cells of the new individual are directly derived. In all such cells the double chromosome number is present. Similar events take place in the maturation and fertilization of animal eggs in general.

Now, when the egg of a black guinea-pig is fertilized with the sperm of a white one, or vice versa, proto-plasmic constituents unite which in one case are able to produce a black coat, in the other a white one. These constituents, whatever they are, evidently separate from each other and pass into different cell products when the germ cells of the cross-bred individual ripen. It seems natural to suppose that the separation occurs at the reduction of the chromosomes from the double to the single condition. Half the sperms, accordingly, of the cross-bred black individual bear black, half white, none both; and the same is true of the eggs (Fig. 20). Experiment proves conclusively that this is so. Blackness and whiteness behave in crosses like indivisible units. They may be brought together repeatedly in crosses, but always separate again at the maturation of the gametes. We call them unit-characters. Black is a positive unit (presence of black pigment), white its corresponding negative (absence of black pigment).

Other unit-characters are quite independent, in their inheritance, of black and white. Thus, the coat of a 
The Method of Evolution

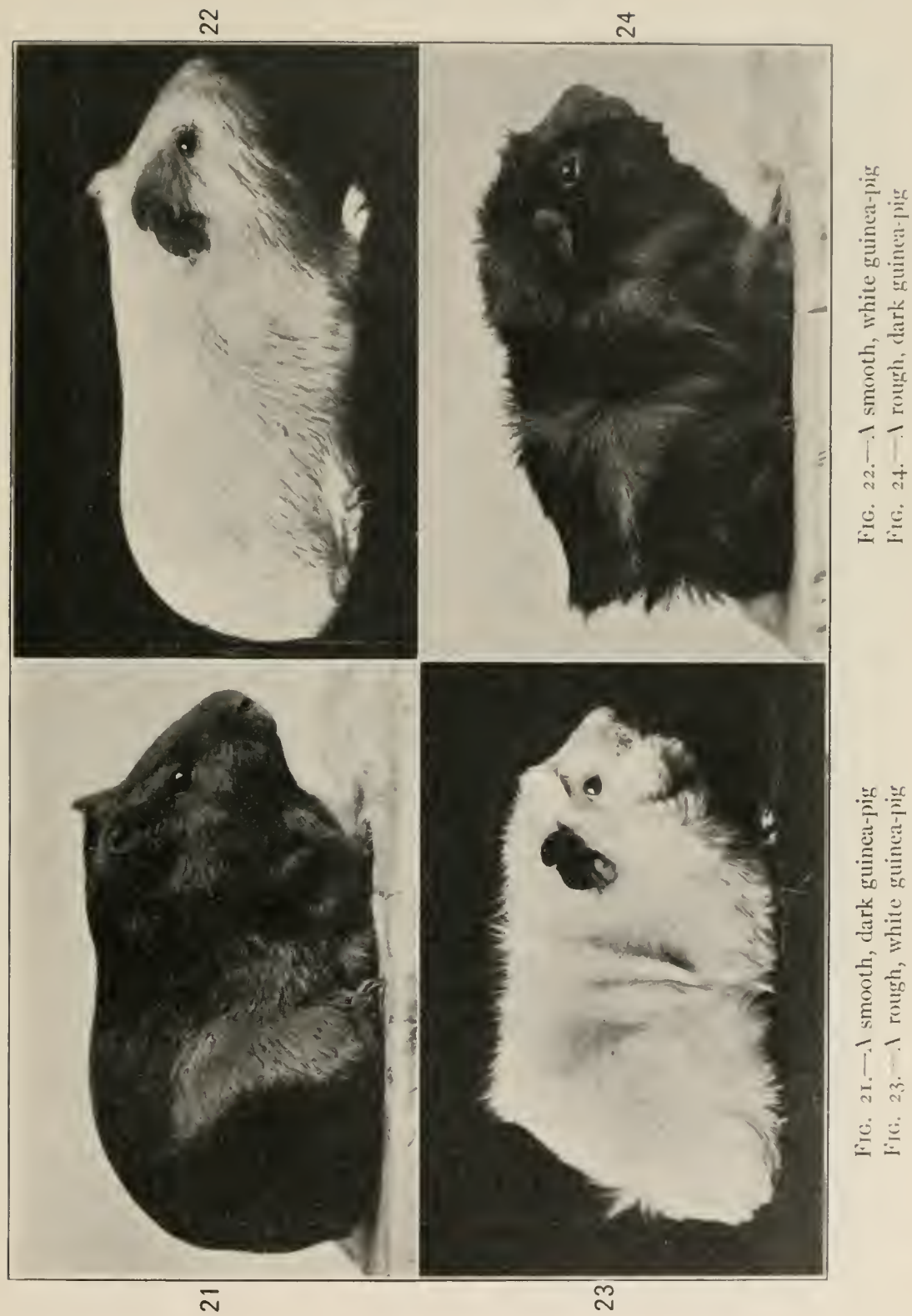




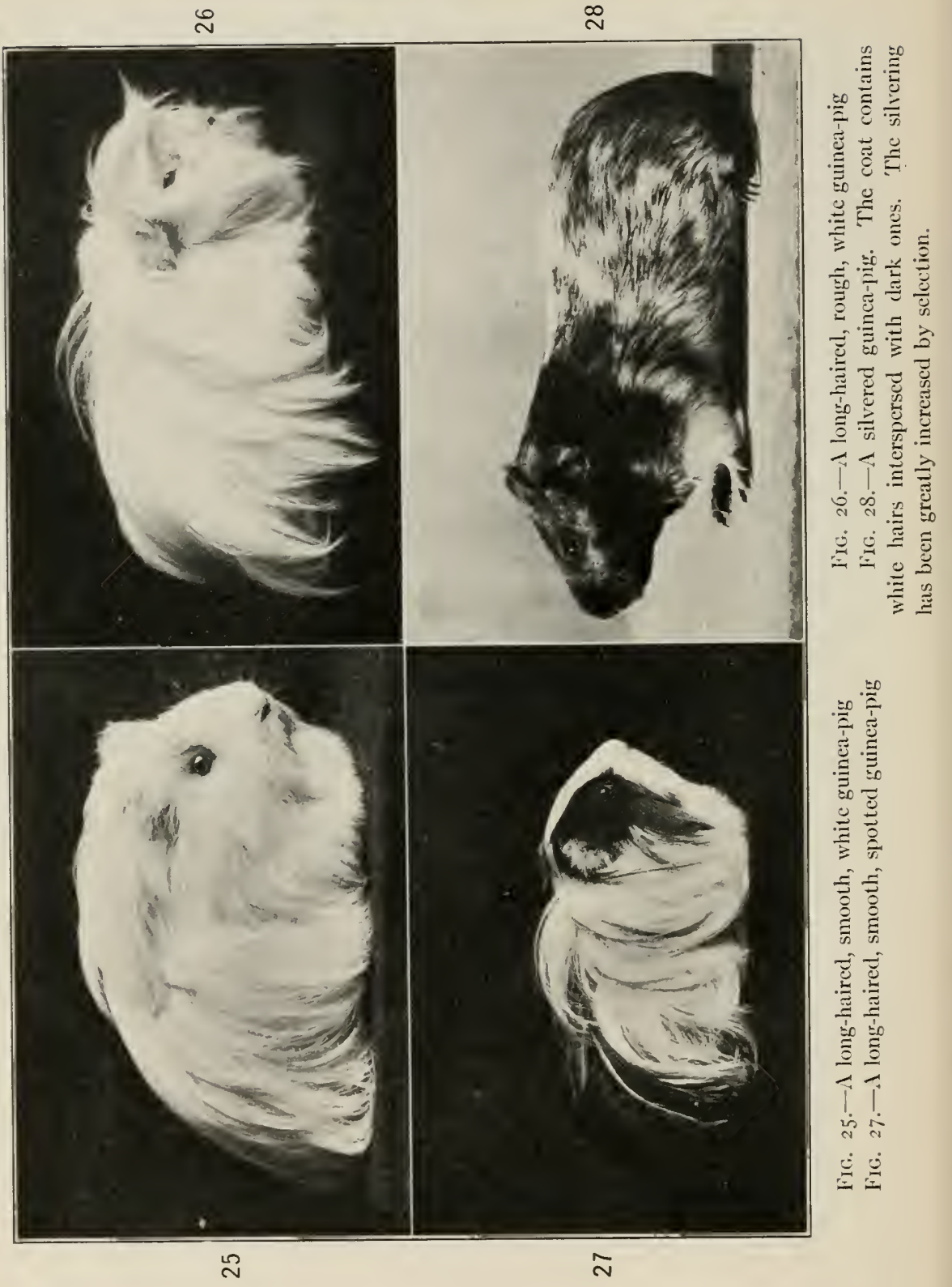


guinea-pig may be either rough or smooth (Figs. $2 \mathrm{I}-24$ ). Rough is a unit-character dominant over smooth in crosses, and among the second generation offspring from such a cross occur three rough individuals to one smooth one. If the rough parent is white (Fig. 23) and the smooth one dark (Fig. 2I), the parents differ in two unit-characters, and the sequel shows that these are independent units. For although the immediate offspring are all dark and rough (Fig. 24), the next generation contains four sorts of individuals, representing all possible combinations of the two alternative pairs of units: (I) smooth dark, like one grandparent (Fig. 2I); (2) rough white, like the other (Fig. 23); (3) rough dark, like the parents (Fig. 24); and (4) smooth white, a new combination (Fig. 22).

Again, length of the hair is independent of its color or roughness (Figs. 25-27). A short-haired colored animal mated with a long-haired white one produces only shorthaired colored offspring, which, bred inter se, produce in the next generation young of four sorts: (I) long white; (2) short dark (3) long dark, and (4) short white.

Recombinations in such ways can be accounted for if we suppose each different unit-character to have its basis in a different material body within the cell, perhaps in a different chromosome or part chromosome. Thus, suppose hair length to have its basis in one cell structure, which we may represent in a diagram (Fig. 29) by a circle, and suppose hair color to have its basis in another cell structure represented by a square, and suppose further that these two structures are independent of each other in their fusions and segregations; then if we cross a long-haired white $\left(L \mathrm{I}^{*}\right)$ animal with a short-haired dark one $(S D)$, a combination (or zygote) will be formed showing only the dominant 
characters, short and dark hair, but able to transmit the alternative conditions, long and white hair. At reproduction by such an individual, $L$ will separate from $S$, and $I$ will separate from $D$, passing into a different cell product, but it will be a matter of chance whether $L$ is associated with $W^{*}$ as originally, or with $D$. Hence the chances are even for the production of the four kinds of gametes shown in the diagram, $L W, S D, L D$, and $S W$, the visible

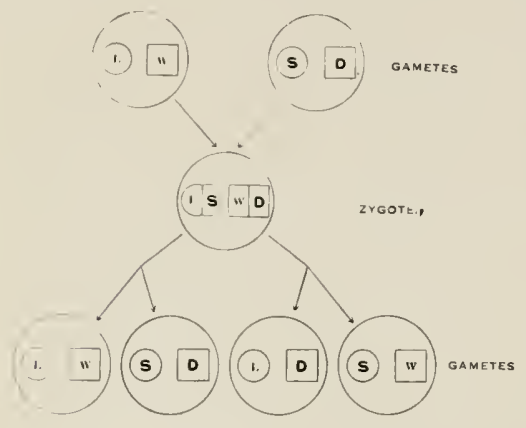

Fig. 29.-Diagram to show the result of crossing a long-haired, white guineapig ( L W ) with a short-haired, dark one (S D). expression of which would produce individuals in character, respectively long white, short dark, long dark, and short white, as actually obtained by experiment.

If the individuals crossed are pure and differ in three particulars, color, length, and roughness of the coat, then their grandchildren will be of eight sorts, representing all possible combinations of three independent unit-character pairs, each of which has its basis in a different material body in the cell.

1 great many of the characters of animals and plants behave as simple units in heredity, yielding a 3 : I ratio of dominant to recessive individuals in the second generation from the cross. I have shown that this is true of certain hair characters in guinea-pigs, namely, blackness, roughness, and length of the hair. We have no idea how numerous such characters are until they happen to be lost in one individual or another. Then a new variation, a sport or mutation, is observed. It is by this means, acci- 
dental loss of simple unit-characters, that the great color variation of domesticated animals has arisen.

We should naturally consider the color character of a wild mouse, rat, or rabbit, to be very simple, for we observe such animals to breed very true to color, but the behavior of the wild type in crosses shows it in reality to be very complex, and to be the result of the simultaneous presence of some half-dozen or more wholly independent unitcharacters. New color varieties have arisen by loss or modification of one or more of these unit-characters. For example, the wild house mouse by simple loss of three independent factors has given rise to seven additional varieties known among fancy mice.

'The gray fur contains black, brown, and yellow pigments disposed in a definite pattern in the individual hairs. Loss of this pattern alone produces the black variety. Loss of black produces the cinnamon variety; loss of both produces the brown or chocolate variety. Loss of the power to produce color, that is loss of some general color factor, produces an albino, whose breeding capacity will vary with the number of other factors which it retains. Several other color factors occur in mice, the loss of which has produced new series of color varieties, but these will suffice to show the process by which new varieties arise through loss of unit-characters.

Simple unit-characters are not confined to the superficial parts of an animal, as for example to its fur. IVe know these superficial characters best probably simply because they are most easily observed.

Loss of horns in cattle behaves as a dominant unitcharacter; likewise in man a shortened condition of the skeleton producing two-jointed instead of three-jointed 
fingers behares as a simple dominant unit-character. A curious affection of the nervous system producing the waltzing condition of Japanese mice, behaves as a recessive unit-character in crosses with the normal condition.

Is all inheritance unit-character inheritance? This question cannot at present be answered fully, but many facts indicate that it is. A large class of seemingly unconformable cases which presented the greatest obstacle to such a view has recently been brought into line with the unitcharacter hypothesis. I refer to cases of blending inheritance, in which the offspring are intermediate between the parents, and this intermediate condition persists into the next generation.

Size and skeletal proportions are inherited apparently in this fashion. It is possible, however, that even in such cases unit-character segregations may really occur, though their presence is obscured because dominance does not occur. For in plants such size segregations have been observed recently by my colleague Dr. East and by others.

A single illustration will suffice. When varieties of maize (or Indian corn) are crossed which differ in size of ear, the hybrid plants bear ears of intermediate size but not more variable than the more variable parent. The second generation offspring, however, are extremely variable, ranging in size from that of the smaller parent variety to that of the larger.

The peculiarity of what we have called blending inheritance lies partly in the entire absence of dominance. In blending inheritance a unit-character represented once in the fertilized egg has only half as much effect as one represented twice. In color inheritance, usually, but not always, a single dose of a unit-character is as effective as a double 
dose in causing the development of the character. In size inheritance, however, the single and double doses probably produce very different effects.

A further apparent difficulty encountered in interpreting blending inheritance as unit-character inheritance lies in the multiplicity of the units involved, so that segregations do not occur into a few discontinuous size classes, but into classes so numerous and differing so little from each other that it is very difficult to distinguish them.

Suppose that in crosses of black with white guinea-pigs, black were represented by two unit-characters $B$ and $B^{\prime}$, instead of by one, residing perhaps in different chromosomes, and that either one of these could by itself produce black color, then a larger proportion than three-fourths of the second generation offspring would be black, namely, $\begin{gathered}15 \\ 16\end{gathered}$. If, further, the presence of a larger number of factors for black produced more black pigment in the fur than a smaller number produced, then we should have gradations of blackness among the second generation offspring as follows: $4,3,2,1,0$.

Add a third factor for black in the supposed cross, located perhaps in a third chromosome, and the pure whites would be reduced to I to 64 of the second generation offspring, while the different gradations or intensities of blackness would become $6,5,4,3,2,1,0$. The occasional white individual would now differ so little from the lightest black one that the two might often be confused, and there would seem to exist all intermediate stages between pure white and pure black, without entire segregation into either. $\mathrm{By}$ selecting for the lightest or the darkest condition within a mixed race of such second generation offspring one would obtain with each selection a larger proportion of extremely 
dark or extremely light individuals, until a pure race was obtained. Further, if the black character should become attached to additional material bodies in the cell (chromosomes or the like), so that it would be represented by additional units, then the occurrence of light-colored progeny would become still rarer, and deeper intensities of blackness than before existed would now occur. Thus selection would become a means for the modification of a character really dependent upon the inheritance of unchanging units. Now this is perhaps what occurs when one seeks to modify size by selection.

There are strong reasons for believing that mendelizing characters can be modified by selection, though this idea is vigorously denied by many Mendelians, as for example by Johannsen. In Johannsen's view, selection can do nothing but sort out variations already existing in a race. I prefer to think with Darwin that selection can do more than this, that it can heap up quantitative variations until they reach a sum total otherwise unattainable, and that it thus becomes creative. I will describe briefly certain experiences of my own which support this idea.

In several cases I have observed characters at first feebly manifested gradually improve under selection until they became established racial traits. Thus in guinea-pigs, the hind-foot commonly bears three toes (Fig. 30, A). But several years ago I observed an individual which had an imperfectly developed fourth toe, similar to that shown in Fig. 30, C. From the descendants of this animal, obtained by inbreeding and selection, was formed a race having well-developed fourth toes (Fig. 30, B) on both hind feet. The extra toe made its appearance poorly developed on the left foot only. About 6 per cent of the 
ofispring of this animal by normal unrelated mothers were polydactylous, but among his offspring were some with better developed fourth toes than the father possessed. Such individuals were selected throughout five successive generations, at the end of which time a good four-toed race had been established. It was found in general that those animals which had best-developed fourth toes transmitted the character most strongly in crosses with unrelated normal animals. The percentage of polydactylous individuals produced in such crosses varied all the way from $\circ$ to roo

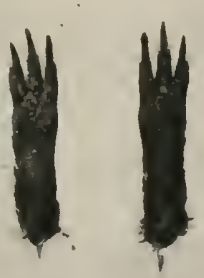

A

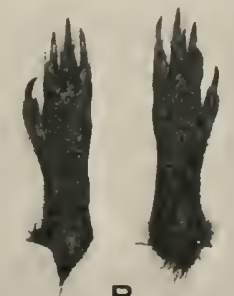

B

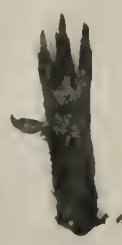

C

FIG. 30.-A, hind-feet of an ordinary guinea-pig; B, of a four-toed guineapig; C, of an imperfectly four-toed guinea-pig.

per cent. By selection this percentage was increased, as was also the degree of development of the fourth toe in crosses.

Another character which made its appearance among our guinea-pigs, at first feebly expressed, was a silvering of the colored fur, due to interspersing of white hairs with the colored ones (Fig. 28). The first individuals observed to have this character bore white hairs on the under surface of the body only. By inbreeding, a homozygous strain of the silvered animals was soon obtained, one in which all the offspring were silvered to a greater or less extent. Selection 
was now directed toward two ends: (I) to secure animals which were free from spots of red or white, a condition which was present in the original stock; and (2) to secure extensive and uniform silvering on a black background. In both these objects good progress has been made. We have animals which are silvered all over the body except on a part of the head, and the percentage of such wellsilvered individuals is relatively high.

A more extensive selection experiment is one in which I have been assisted by Dr. John C. Phillips (Figs. 3 I and 32).

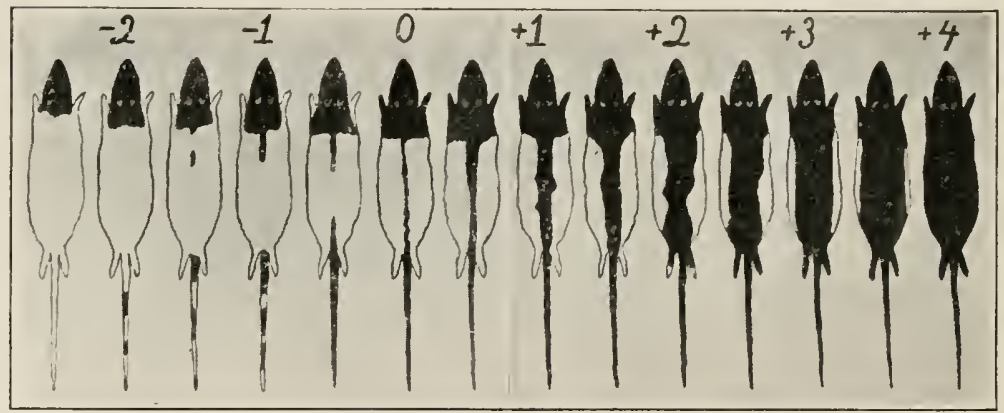

FIG. 3I.-Diagram showing variation in the color pattern of hooded rats. Numerals indicate arbitrary grades used.

Selection in this case has been directed toward a modification of the color pattern of hooded rats, a pattern which is known to behave as a recessive Mendelian character in crosses with either the self (totally pigmented) condition or the so-called Irish (white bellied) condition found in some other rats. The extreme range of variation among our hooded rats at the outset of this experiment is indicated by the grades -2 and +3 of Fig. 3 I. Selection was now made of the extreme variates in either direction and these were bred separately. Two series of animals were thus 
established: one of narrow-striped animals, minus series; the other of wide striped, plus series. In each generation the most extreme individuals were selected as parents; in the narrow series, those with narrowest stripe; in the wide series, those with widest stripe.

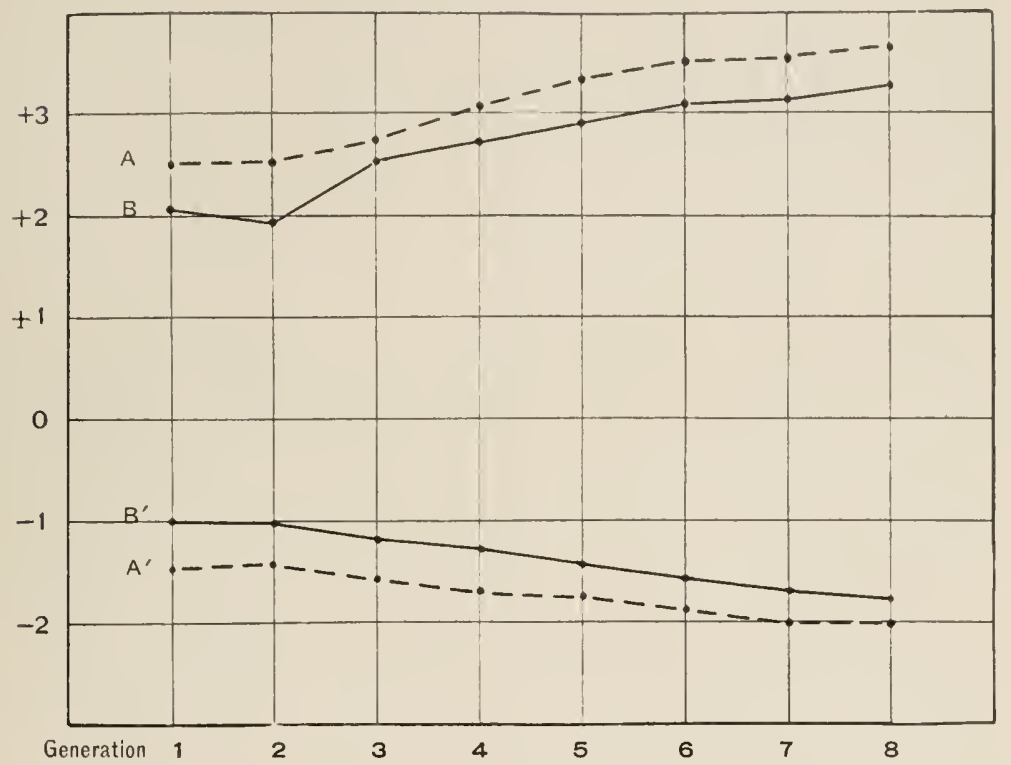

FIG. 32.-Chart showing effects of selection in eight successive generations upon the color pattern of hooded rats. $A, A^{\prime}$, average condition of selected parents; $B, B^{\prime}$, of their offspring.

The result of the selection is shown graphically in Fig. 32 (compare Table I). The offspring in the narrow series became with each generation narrower; those in the wide series became with each generation wider, with a single exception. In the second generation the wide stock was enlarged by the addition of a new strain of animals. This caused a temporary falling off in the average grade of the young, the two series overlapping for that generation. No 
new stock was at any other time introduced in either series, the two remaining distinct at all times except in the second generation. It will be observed that a change in the average grade of the parents is attended by a corresponding change in the average of the offspring, and likewise in the range of variation in the offspring. The amount of variability of the offspring is not materially affected by the selection, but the average about which variation occurs is steadily changed, as are also the limits of the range of variation.

\section{TABLE I}

Results of Selection for Modification of the Color-Pattern of HOODED Rats

\begin{tabular}{|c|c|c|c|c|}
\hline & Generation & $\begin{array}{c}\text { Average Grade } \\
\text { Parents }\end{array}$ & $\begin{array}{l}\text { Average Grade } \\
\text { Offspring }\end{array}$ & $\begin{array}{l}\text { Number of } \\
\text { Offispring }\end{array}$ \\
\hline Plus series & 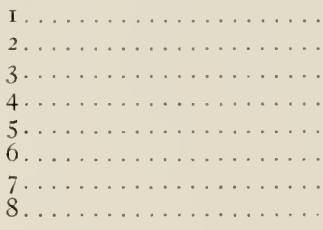 & $\begin{array}{l}2.50 \\
2.5 \mathrm{I} \\
2.73 \\
3.09 \\
3.33 \\
3.5 \mathrm{I} \\
3.53 \\
3.65\end{array}$ & $\begin{array}{l}2.05 \\
1.92 \\
2.51 \\
2.72 \\
2.90 \\
3.09 \\
3.14 \\
3.30\end{array}$ & $\begin{array}{r}\text { I } 50 \\
47 \mathrm{I} \\
34 \mathrm{I} \\
444 \\
610 \\
834 \\
874 \\
9 \mathrm{I}\end{array}$ \\
\hline Total. . & & & & 3,8 I 5 \\
\hline Minus series & $\begin{array}{l}1 \\
2 \ldots \\
3 \ldots \\
4 \ldots \\
5 \\
5 \\
6 \ldots \\
7 \ldots \ldots \\
8 \ldots \ldots\end{array}$ & $\begin{array}{l}\text { I. } 46 \\
\text { I. } 4 \text { I } \\
\text { I. } 56 \\
\text { I. } 69 \\
\text { I. } 73 \\
\text { I. } 86 \\
2.00 \\
2.03\end{array}$ & $\begin{array}{l}\text { I. } 00 \\
\text { I. } 07 \\
\text { I. I } 8 \\
\text { I. } 28 \\
\text { I. } 4 \text { I } \\
\text { I. } 56 \\
\text { I. } 70 \\
\text { I. } 78\end{array}$ & $\begin{array}{r}55 \\
132 \\
195 \\
329 \\
701 \\
1,252 \\
1,544 \\
713\end{array}$ \\
\hline Total. & & & & 4,921 \\
\hline
\end{tabular}

The interesting feature of this experiment is the production, as a result of selection, of wholly new grades, in the narrow series of animals having less pigment than any known type other than the albino; in the wide series, of 
animals so extensively pigmented that they would readily. pass for the "Irish type," which has white on the belly" only, but which is known to be in crosses a Mendelian alternative to the hooded type. By selection we have practically obliterated the gap which originally separated these types, though selected animals still give regression toward the respective types from which they came. But this regression grows less with each successive selection and ultimately should vanish, if the story told by these statistics is to be trusted. As yet there is no indication that a limit to the effects of selection has been reached.

From the evidence in hand we conclude that Darwin was right in assigning great importance to selection in evolution; that progress results not merely from sorting our particular combinations of large and striking unitcharacters, but also from the selection of slight differences in the potentiality of gametes representing the same unitcharacter combinations.

Accordingly we conclude that unit-characters are not unchangeable. They can be modified, and these modifications come about in more than a single way. Occasionally. a unit-character is lost altogether or profoundly modified at a single step. This is mutation. But more frequent and more important, probably, are slight, scarcely noticeable modifications of unit-characters that afford a basis for a slow alteration of the race by selection. Mutation, then, is true, but it is a half-truth; selection is the other and equally important half of the truth of evolution, as Darwin saw it and as we see it. 


\section{CHAPTER IV}

\section{HEREDITY AND SEI}

The value of a domesticated animal often depends in considerable measure on its sex. Therefore, if a means could be devised for controlling the sex of offspring, it would be of great economic value to the breeder. Endless attempts have been made to do this, and occasionally a claim of success has been made, but none of these claims has withstood the test of critical analysis or experiment. The hypotheses advanced to explain how sex may be controlled have been of the most varied character. In some the determination of sex has been supposed to inhere in the nature of the parents, in others it is referred to conditions of the gametes themselves.

Relative age or vigor of the parents has been supposed to influence sex in various ways. The same idea has been advanced regarding the gametes themselves, it being supposed that early or late fertilization of the egg might influence its sex. Experimental evidence, however, as to these several hypotheses is wholly negative, when one eliminates other possible factors from the experiment. Everything points to the conclusion that sex rests in the last

${ }^{I}$ In reading this chapter, the following definitions should be kept in mind.

Gamete: a mature reproductive cell capable, on uniting with another gamete, of forming a new individual.

Zygote: the new individual formed by the union of two gametes.

Homozygote: an individual formed by the union of two gametes of like character as regards heredity:

Hctcrozygote: an individual formed by the union of two gametes of unlike character as regards heredity. 
analysis upon gametic differentiation, just as the color of a guinea-pig in a mixed race of blacks and whites depends upon whether the gametes which unite to produce it carry black or white. As the heterozygous black guinea-pig forms black-producing and white-producing gametes in equal numbers, so there is reason to think male-producing and female-producing gametes are formed in equal numbers by the parent, in many cases at least. But is it not possible that there may exist individuals which produce the two sorts of gametes in unequal numbers, and so would have a tendency to produce more offspring of one sex than of the other? Perhaps so, though we have no evidence that such a condition, if it does exist, is transmitted from one generation to another. On this point I made experimental observations upon guinea-pigs extending over a series of years. Oftentimes I found an individual that produced more offspring of one sex than of the other, but this was probably due merely to chance deviations from equality. I could get no evidence that the condition was inherited, though the experiment was continued through as many as seven generations, including several hundred offspring.

The essential difference between a female and a male individual is that one produces eggs, the other sperm. AlI other differences are secondary and dependent largely upon the differences mentioned. If in the higher animals (birds and mammals) the sex glands (i.e., the egg-producing and sperm-producing tissues) are removed from the body, the superficial differences between the sexes largely disappear. In insects, however, the secondary sex-characters seem to be for the most part uninfluenced by presence or absence of the sex glands. Their differentiation occurs independently though simultaneously with that of the sex glands. 
The egg or larger gamete (the so-called macro-gamete) in all animals is non-motile and contains a relatively large amount of reserve food material for the maintenance of the developing embryo. This reserve food material it is the function of the mother to supply. In the case of some animals, for example flatworms and mollusks, the food supply of the embryo is not stored in the egg cell itself, but in other cells associated with it, and which break down and supply nourishment to the developing embryo derived from the fertilized egg. Again, as in the mammals, the embryo may derive its nourishment largely from the maternal tissues, the embryo remaining like a parasite within the maternal body during its growth, feeding by absorption. But in all cases alike the mother supplies the larger gamete and the food material necessary to carry the zygote through its embryonic stages. The father, on the other hand, furnishes the bare hereditary equipment of a gamete, with the motor apparatus necessary to bring it into contact with the egg cell, but without food for the developing embryo produced by fertilization. The gamete furnished by the father is therefore the smaller gamete, the so-called micro-gamete.

From the standpoint of metabolism, the female is the more advanced condition; the female performs the larger function, doing all that the male does in furnishing the material basis of heredity (a gamete), and in addition supplying food for the embryo. As regards the reproductive function, the female is the equivalent of the male organism, plus an additional function, that of supplying the embryo with food. When we come to consider the structural basis of sex, we find reasons for thinking that here, too, the female individual is the equivalent of the male plus an additional element. The conclusion has very 
naturally been drawn that if a means could be devised for increasing the nourishment of the egg or embryo, its development into a female should be thereby insured, while the reverse treatment should lead to the production of a male. But in practice this a-priori expectation is not fulfilled. Better nourishment of the mother may lead to the production of more eggs, but not of more female offspring, as has repeatedly been demonstrated by experiment. Also poor nutrition of the mother may diminish the number of eggs which she liberates, but will not increase the proportion of males among the offspring produced.

An excellent summary of evidence on this point was made by Cuénot in I9oo. Attempts to influence the sex of an embryo or larva by altered nutrition of the embryo or larva itself have proved equally futile. Practically the only experimental evidence of value in favor of this idea has been derived from the study of insects, and this is capable of explanation on quite different grounds from those which first suggest themselves. It has sometimes been observed, as by Mary Treat for example, that a lot of insects poorly fed produce an excess of males. In such lots, however, the mortality is commonly high, and more females die than males, because the female is usually larger and requires more food to complete its development. The fallacy in concluding from such evidence that scanty nutrition causes individuals which would otherwise become females to develop into males was indicated years ago by Riley. Nevertheless an argument for the artificial control of sex based on such evidence is from time to time brought forward, as, for example, a few years since by Schenk. The latest adrocate of sex control by artificial means is an Italian, Russo (I909). He claims in the case of rabbits that by 
feeding the mother on lecithin or by injections of lecithin, the proportion of female births may be increased. His evidence in support of this claim is, however, wholly inadequate, and two independent repetitions of his experiments, made by Basil in Italy and by Punnett in England, have given entirely negative results.

An alternative hypothesis concerning the determination of sex has been steadily gaining ground during the last ten years, that sex has its beginning in gametic differentiation and is finally determined beyond recall in the fertilized egg by the nature of the uniting gametes. Instructive in this connection is a study of parthenogenesis, reproduction by unfertilized eggs. But before entering upon this, it may be well to review briefly the changes which regularly take place in the egg which is to be fertilized, and compare with this the changes which occur in eggs not to be fertilized.

In each cell of the ordinary animal there occurs a characteristic number of bodies called chromosomes. We do not know that they are any more important than other cell constituents, but we know their history better. These are contained in the nucleus of the cell, and at the time of nuclear division they are found at the equator of the division spindle. There each of them regularly splits in two, and one derivative goes to either end of the spindle, and so into one of the daughter nuclei. Thus each new nucleus has, as a rule, the same chromosome composition as the nucleus from which it was derived.

But the egg which is to be fertilized undergoes two nuclear divisions in succession, in only one of which do the chromosomes split. In the other division the chromosomes separate into two groups without splitting, and each group goes into a different cell product. Consequently, 
in each of these products the number of chromosomes is reduced to half what it is in the cells of the parental body. Thus in the egg of the mouse, by maturation, the number of chromosomes becomes reduced from about twenty-four to about twelve.

Similar changes occur in the developing sperm cell (Fig. 33, upper row). Starting with the double or 2.1 chromosome number, there are formed by two nuclear divisions, with only one splitting of chromosomes, four cells, each with the reduced or simplex number of chromosomes, $\mathrm{N}$. From each of these four cells arises a tadpole-like sperm. Consequently, when the sperm enters the egg at fertilization it brings in a group of $N^{\top}$ chromosomes (in the mouse apparently I2),
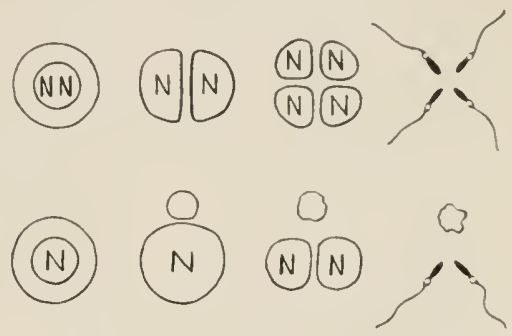

FIc. 33.-Top row, normal spermatogenesis; lower row, spermatogenesis of simplex male.

which, added to the egg contribution of $\mathrm{N}$ chromosomes, brings the number in the new organism again up to $2 . \mathrm{Y}^{\circ}$ (in the mouse 24).

Now, as regards the maturation of parthenogenetic eggs, those which are to develop without having been fertilized, three categories of cases deserve separate discussion. The simplest of these in many respects is found among the social hymenoptera (ants, bees, and wasps) (see Fig. 34. left column). The eggs are, so far as we can discover, all of a single type. They undergo maturation in the manner already described, the chromosomes being reduced to the $N$ or simplex number. The eggs of most animals, after they have undergone reduction, are incapable of develop- 
ment unless fertilized, but those of the hymenoptera may develop either fertilized or unfertilized. In the former case a female is produced, in the latter a male. The simplex or $Y$ condition is in this case the male, the duplex or $2 X$

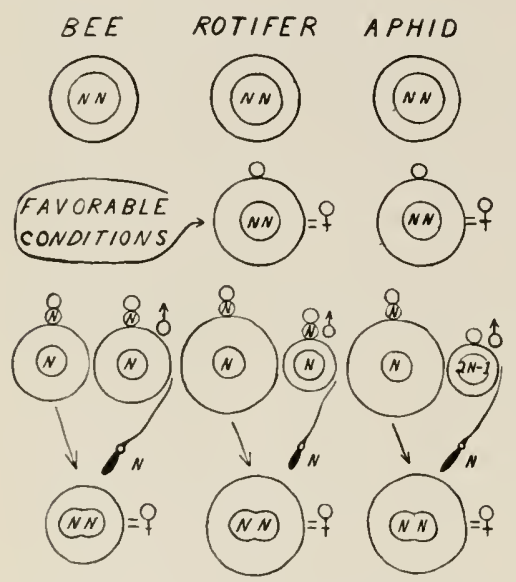

FIG. 34.--Sex determination in parthenogenesis. Top row, nuclear condition of the parthenogenetic mother; second row, of her eggs when they develop without nuclear reduction, having formed a single polar cell; third row, condition of the eggs after complete maturation-the unfertilized egg in each case produces a male; fourth row, nuclear condition of the fertilized egg, always a female. condition is the female, naturally the one of higher metabolic activity, the one which forms the macrogametes.

There is a peculiarity in the maturation of the sperm cells of male animals of this sort (Fig. 33, lower row). The cells of the male are in this case already in the reduced or simplex condition, $N$. In the production of the sperms the reducing division is omitted so far as nuclear components are concerned, so that each sperm formed contains the full simplex chromosome number, $N$. If it were less, the gamete formed would perhaps not be capable of transmitting all the hereditary characteristics of an individual.

A second category of cases (Fig. 34, middle column) is represented by such simple aquatic organisms as rotifers and small crustacea, like Daphnia. In these parthenogenesis occurs exclusively, when the food supply is very abundant and conditions otherwise favorable, whereas 
reproduction by fertilized eggs occurs only when external conditions, including food supply, are not good. Under favorable conditions only female offspring are produced. The conclusion has naturally but erroneously been drawn that good nutrition in itself favors the production of females in animals generally, which is not true. The egg produced by Daphnia, or by a rotifer, under optimum conditions docs not undergo reduction (Fig. 34, second row). It remains in the $2 \mathrm{~N}$ condition, forming but a single polar cell. It is therefore unprepared for fertilization, and in fact it is not fertilized. Its sex is like that of the animal which formed it, female. Under unfavorable conditions, however, the eggs of the rotifer and of Daphnia do not begin development until they have undergone maturation. They are also of two sizes (Fig. 34, third row)-small eggs, which derelop without fertilization and which form males, and large eggs, which require fertilization, and which form females. In this category of cases, as in that of the hymenoptera, the egg which develops in the $2 N$ condition, either from failure of reduction to occur in maturation or from fertilization following reduction, forms a female; whereas the egg which develops in the $\mathrm{Y}$ condition forms a male.

In a third category of cases there is a quantitative difference in chromatin between male and female, just as in the foregoing cases, but this does not amount to a whole set of chromosomes, $N$, but to only a partial set, one or two chromosomes (Fig. 34, right column). This category of cases occurs in plant lice (aphids and phylloxerans); evidence of its existence rests chiefly on recent observations made by von Baehr and Morgan. Females are formed by parthenogenesis without reduction, occuring under farorable conditions, just as in the case of rotifers. Females are also 
formed by fertilization following reduction under unfavorable conditions, just as in rotifers. In both cases the female is $2 . \mathrm{T}$. Males arise only by parthenogenesis under unfavorable conditions, just as in rotifers, but the reduction which occurs before development begins is partial only. A whole set, $\mathbf{T}$, of chromosomes is not eliminated in maturation, but only I or 2 chromosomes. Hence the male condition here

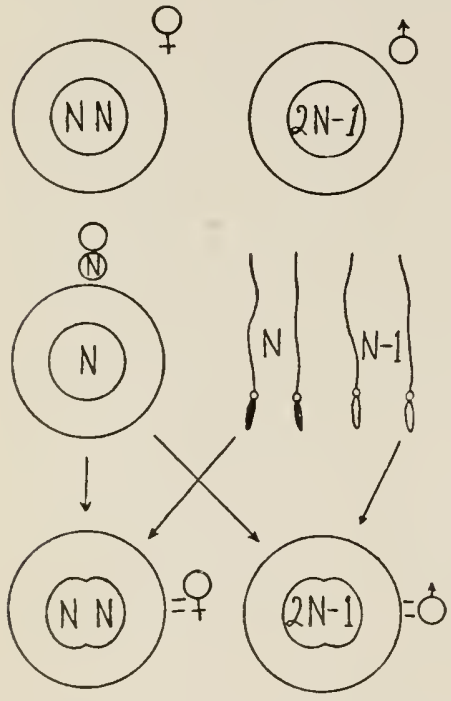

FIG. 35.-Diagram of sex determination when the female is homozygous, the male heterozygous. is $2 N-\mathrm{I}$ or -2 . The condition of the gametes formed, however, is $N$ in both sexes. In spermatogenesis, division of the germ cells takes place into $N$ and $N$ - I daughter cells, but the latter degenerate (like the non-nucleated cells of the bee and wasp), and only the former produce spermatozoa. Hence in fertilization only $2 \mathrm{~N}^{\top}$ zygotes are produced, which are invariably female.

Summarizing the three categories described, we may say that in all known cases of parthenogenesis, the female is in the duplex $(2 \mathrm{~T})$ condition, the male in the simplex $\left(\mathrm{I}^{*}\right)$ or partially duplex condition $\left(2 \mathrm{~N}-\mathrm{I}\right.$, or $\left.2 \mathrm{~N}^{\top}-2\right)$. The female in all cases has the greater chromatin content.

In a great many insects and other arthropods, which are not parthenogenetic, it is known that, although the male, like the female, develops only from a fertilized egg, nevertheless the male possesses fewer chromosomes than 
the female. In such cases the female forms, as in cases of parthenogenesis, only $Y$ gametes, but the male forms gametes of two sorts, $\mathrm{N}$ and $\mathrm{N}-\mathrm{I}$ or $\mathrm{N}^{\top}-2$ (Fig. 35). In consequence zygotes of two sorts result, those which are $2 . \mathrm{T}$, female, and those which are $2 \lambda-\mathrm{I}$ or $2 \mathrm{~N}^{\top}-2$, male. Thus in the squash bug, Anasa tristis, according to Wilson, the mature egg contains i c chromosomes, the spermatozoa either so or I I chromosomes, the two sorts being equally numerous.

Egg II + sperm ir produces a zygote $22\left(2 \mathrm{~N}^{\circ}\right)$, a female

Egg I I +sperm ro produces a zygote 2I $(2 \lambda-\mathrm{I})$, a male

$Y$ in this species=II; $2 \Lambda^{\top}=22$, the female; $2 \Lambda^{\top}-\mathrm{I}=2 \mathrm{I}$, the male. Males and females are therefore approximately equal in number, as in most animals where the two sexes are not subject to unequal mortality. In the Mendelian sense the female is in such cases a homozygote, the male a heterozygote. The sex of an individual in such cases depends upon which sort of a sperm chances to enter the egg.

But the experimental evidence indicates that both as regards sex and as regards heritable characters correlated with sex, these relations may in some cases be reversed, the female being heterozygous, the male homozygous. In such cases there is reason to think that structurally the male is $2 . N$ but the female $2 N+$. That is, the female is still the equivalent of the male plus some additional element and function. A structural basis in the chromosomes for such a condition has been described by Baltzer in the case of the sea-urchin. He found the regular duplex number of chromosomes in the male; but in the female, while the number was the same, one of the chromosomes was larger than its mate, having an extra or odd element attached to it. In such a 
case the gametes formed by the male would all be $N$, but those formed by the female would be of two sorts equally numerous, viz., $N$ and $N+$ (Fig. 36). $\operatorname{Egg} N$ fertilized by sperm $N$ would produce a zygote $2 N$, a male; egg $N+$ fertilized by sperm $N$ would produce a zygote $2 N^{2}+$, a female. Hence, here as in other animals, the sexes would
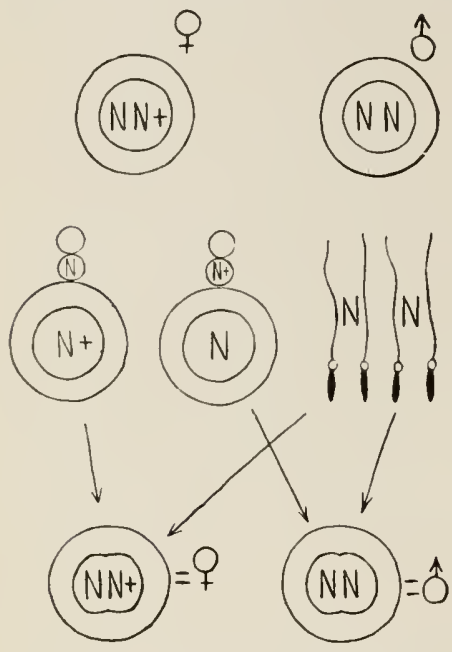

Fig. 36.-Diagram of sex determination when the female is heterozygous, the male homozygous. be approximately equal, but the sex of a particular individual would depend upon which sort of egg gave rise to it.

Upon the existence, as in the foregoing cases, of an unpaired or odd structural element in the egg, may perhaps depend the explanation of a curious sort of heredity known as sex-limited heredity.

Everyone who knows anything about poultry is acquainted with the popular American breed called barred Plymouth Rock. In this breed the feathers are marked with alternate bars of darker and lighter black. Pure barred Rocks breed true, but when crossed with other breeds, the male proves to be homozygous, the female heterozygous in barring. For the male Rock crossed with a non-barred breed produces only barred offspring in both sexes, but the female Rock crossed with the same non-barred breed produces offspring approximately half of which are barred, the other half being non-barred. Further, the barred individuals in this cross 
are invariably males, the non-barred ones being females. Accordingly, the distribution of barring and non-barring in the cross is sex limited.

The barred offspring produced by a cross between barred Plymouth Rocks and a non-barred breed, whether those offspring are males or females, prove to be heterozygous in barring, as we should expect, the barring factor having been received only from one parent, the barred one. Further, the non-barred offspring produced by a barred Rock female crossed with a non-barred breed, do not transmit barring, hence they are pure recessives as regards barring. Hence, also, we are forced to conclude, as already suggested, the female of the pure barred Rock breed is heterozygous as regards barring, and transmits the character only to her male offspring, her female offspring (if the father is non-barred), neither being themselves barred nor being able to transmit barring.

A pure Plymouth Rock race breeds true to barring merely because all its males are pure, for the females are not pure. This is shown by the following experiment. If a heterozygous barred male, produced by a cross between a Rock and a non-barred breed, is crossed with barred females, either those of a pure Rock race or those produced by a cross, the result is the same. The male offspring are all barred; the females, half of them barred, half nonbarred. This result shows that all barred females alike are heterozygous in barring.

Sex-limited inheritance such as this finds at the present time its most probable explanation in the existence in the egg of an extra or plus element never found in the sperm, this element pairing with the sex-limited character in the reduction division. Thus, in the barred Rock, calling 
barring $B$, the male of pure race is plainly $B B$ and every sperm is $B$. But the female clearly contains only one $B$ and cannot be made to contain two. Perhaps a second $B$ is kept out by some structural element, $X$, the distinctive structural element of the female individual. Then the eggs will be of two sorts: $B$ and $X$ (Fig. 37). Since the sperms are all $B$, the first type of egg when fertilized will

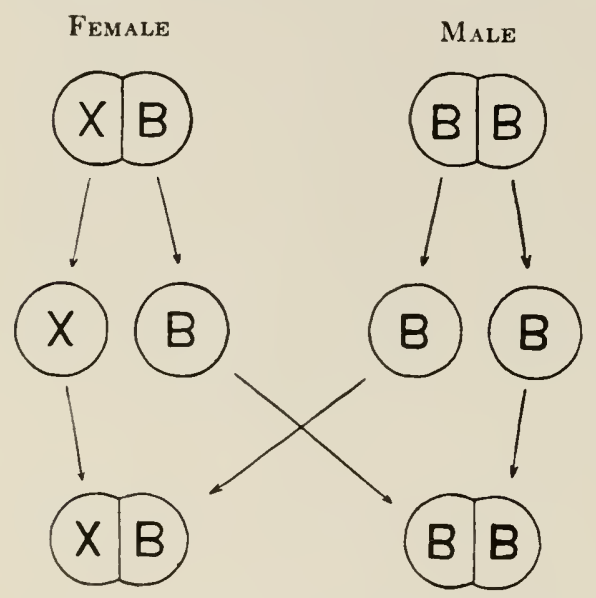

FIG. 37--Diagram of sex-limited inheritance when the female is a heterozygote, as in barred fowls. $\mathrm{X}$, female sex determiner; B, barring. contain $B B$, a homozygous barred individual and a male, since it lacks $X$; the second type will contain $B X$, a bird heterozygous in barring, and a female, since it contains $X$. This agrees with the experimental result.

A heterozygous barred male will form two kinds of sperm, only one of which will contain $B$. If such a male be mated with a barred female, four sorts of zygotes should result as follows:

Gametes of heterozygous barred male $=B$ and -

Gametes of barred female $=B$ and $X$

Zygotes $=B \cdot B$ (homozygous barred male) $B$. - (heterozygous barred male), $B \cdot X$ (barred female), and $-\cdot X$ (non-barred female).

The observed result of this cross accords fully with the foregoing expectation.

The sex-limited inheritance of barring in fowls may be explained, as we have just seen, on the assumption that the 
female is the heterozygous sex. The same is true of sexlimited inheritance in canary birds and in the moth, Abraxas, according to Bateson and Doncaster. But these relations are exactly reversed in the pomace fly, Drosophila ampelophila, according to Morgan.

In Drosophila the female is apparently homozygous as regards some cell structure, $I$, which in the male is never represented more than once. Accordingly, the formula of the female is in such cases $X I$; that of the male, $\mathrm{I}-$. Now the sexlimited characters in Drosophila seem to be bound up with the $X$ structure, not repelled by it, as is barring in fowls. Accordingly, a sex-limited character may be represented twice in the female Drosophila, but only

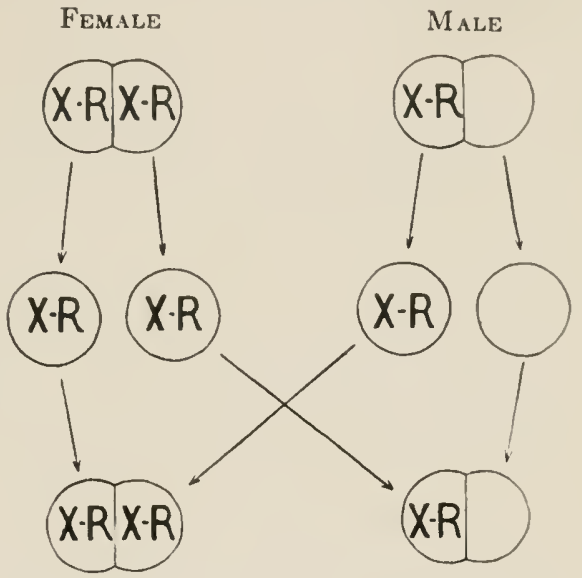

FIG. 38.-Diagram of sex-limited inheritance when the female is a homozygote, as in the redeyed Drosophila. X, sex determiner; R, red eyes.

once in the male; or in other words, the female may be homozygous as regards a sex-limited character, but the male can only be heterozygous (Fig. 38).

Drosophila normally has red-eyes, but the redness of the eye is a distinct unit-character, sex limited in heredity. Further, males are regularly heterozygous in this character, while females are homozygous. For Morgan has obtained a race in which the eyes are white, owing to the loss of the red character; and reciprocal crosses of this race with 
ordinary red-eyed animals yield different results. The red-eyed female crossed with a white-eyed male produces only red-eyed offspring, but the red-eyed male crossed with a white-eyed female produces offspring only half of which are red eyed, viz., the females, whereas the males are white eyed.

These different results in the two cases apparently come about as follows:

FIRST CASE

Gametes of red-eyed female $=X-R$ and $X-R$

Gametes of white-eyed male $=X$ and -

Zygotes $=X \cdot X-R$ (red-eyed female), and $-\cdot X-R$ (red-eyed male).

\section{SECOND CASE}

Gametes of white-eyed female $=X$ and $X$

Gametes of red-eyed male $\quad=X-R$ and -

Zygotes $=X \cdot X-R$ (red-eyed female), and $-\cdot X$ (white-eyed male).

A short condition of the wings in Drosophila, which renders the animal incapable of flight, is likewise sex limited in heredity, as has been shown by Morgan. By crossing two races of Drosophila, each of which possessed a different sex-limited character, Morgan has been able to combine the two characters in a single race. Thus was obtained a race both white eyed and short winged. The synthesis cannot be made originally in a male individual, but only in a female. For only in the female can the two characters be brought together, each associated with a different $X$, since in the male only one $X$ is present. Although each sex-limited character seems to be attached to or bound up with an $\mathrm{Y}$ structure, it evidently has a material basis distinct from $\mathrm{I}$. Otherwise it would not 
be possible for the character to leave one $\mathrm{Y}$ and attach itself to the other, as apparently takes place in the female when the combination of two sex-limited characters in the same gamete is secured through a cross. The combination is apparently secured in this way:

Gametes uniting, $X-R$ and $X-L$

Zygote formed, $X-R \cdot X-L$

Its gametes, $X-R$ and $X-L$, or $X-R-L$, and $X$.

One of the uniting gametes, $Y-R$, is formed by the red-eyed, short-winged parent; the other, $X-L$, is formed by the long-winged, white-eyed parent. The zygote resulting is a red-eyed individual, since it contains $R$; it is long winged, since it contains $L$; it is a female, since it contains two Xs. Now, its gametes are of four sorts, as indicated. The first two sorts result from simple separation of the two $X \mathrm{~s}$, each with its associated character, $R$ in one case, $L$ in the other. But the third sort could result only from the attachment of $R$ and $L$ to the same $X$, leaving the other $X$ without either $R$ or $L$ as the fourth kind of gamete. This kind, which transmits neither red eyes nor long wings, would represent the new gametic combination-white eyed and with short wings.

The experimental evidence that gametes of these four sorts are formed by females of the origin described is as follows: When such a female is mated with a long-winged, white-eyed male, there are obtained female offspring, all of which are long winged, but half of them are red eyed, half white eyed. The male offspring, however, are of four sorts, viz., red short, white long, red long, and white short. This result harmonizes with the hypothesis adranced. For if the gametes of the female are $I-R, I-L, X-R-L$, 
and $X$, and those of the male are $X-L$ and - , then the following combinations should result:

$X-L \cdot X-R, \quad$ red long female

$X-L \cdot X-L$, white long female

$X-L \cdot X-R-L$, red long female

$X-L \cdot X$, white long female

$-\because X-R$, red short male

$-X-L$, white long male

$-X-R-L$, red long male

$-X, \quad$ white short male

This expected result accords with that actually obtained by Morgan.

Color-blindness in man is a sex-limited character, the inheritance of which resembles that of white eyes or short wings in Drosophila, rather than of barring in poultry.

Color-blindness is much commoner in men than in women. A color-blind man, however, does not transmit color-blindness to his sons, but only to his daughters, the daughters, however, are themselves normal provided the mother was; yet they transmit color-blindness to half their sons. A color-blind daughter could be produced, apparently, only by the marriage of a color-blind man with a woman who transmitted color-blindness, since the daughter to be color-blind must have received the character from both parents, whereas the color-blind son receives the character only from his mother.

Color-blindness is apparently due to a defect in the germ cell-absence of something normally associated there with an $\mathrm{Y}$-structure, which is represented twice in woman, once in man. Color-blindness follows, therefore, in transmission, the scheme shown in Fig. 38 . 
If, as has been suggested, the determination of sex in general depends upon the inheritance of a Mendelian factor differentiating the sexes, it is highly improbable that the breeder will ever be able to control sex. Male and female zygotes should forever continue to be produced in approximate equality, and consistent inequality of male and female births could result only from greater mortality on the part of one sort of zygote than of the other. Only in parthenogenesis can man at will control sex, and until he can produce artificial parthenogenesis in the higher animals, he can scarcely hope to control sex in such animals.

Negative as are the results of our study of sex control, they are perhaps not wholly without practical value. It is something to know our limitations. We may thus save time from useless attempts at controlling what is uncontrollable and devote it to more profitable employments. 



\section{EDWARD MURRAY EAST}

Assistant Professor of Experimental Plant Morphology Harvard University 



\section{CHAPTER V}

\section{INHERITANCE IN THE HIGHER PLANTS}

The general acceptance of the theory of organic evolution made necessary a new botany as well as a new zoölogy. The artificial classification of plants in general use a halfcentury ago has been replaced by one based upon the natural relationships that have linked together plant groups during their progressive changes. The need of new facts in the prosecution of this great work has played no small part in the rise of plant morphology, histology, physiology, and ecology to well-deserved places of eminence in science. But in so far as the valuable subject-matter of these botanical sciences have touched or been touched by conceptions of evolution, they have, in general, simply contributed additional facts which have harmonzied with and supported that great truth already so well established by Darwin.

Such a historical point of view did not fully satisfy the seeker after knowledge. He wished to know why and how these things came about. In the gratification of this curiosity as to the mechanism of heredity, of evolution, and of the changes that occur under domestication, rapid progress has recently been made. The botanist has joined hands with the zoölogist and a new subdivision of the biological sciences-Genetics - has arisen.

As subject-material for use in attacking genetic problems, plants and animals each have advantages and disadrantages; but the results of the botanist and of the zoölogist have been wonderfully harmonious. The same methods are used by both, and the work of the pedigree culturist, the 
histologist, and the biological chemist supplement each other in a way that bespeaks a most hopeful future. We shall discuss some results obtained by the use of the oldest method, that of the garden, or, to give it the more aristocratic name, the pedigree culture.

This method is old, but its most important results are modern. A century and a half ago Kölreuter carried out the first systematic studies in plant hybridization. Through them he was able to show that most reciprocal crosses are identical, and therefore that the pollen grain is as essential and important in determining the characters of a hybrid as is the ovule. Kölreuter, of course, did not know the true process of reproduction, but he came as near the truth as could have been expected at the time by showing that an application of 50 or 60 pollen grains was sufficient for the production of over 30 seeds.

Kölreuter, and a little later Thomas Knight, both found that hybrids were in general more vigorous than either of the parents; and Knight from this fact argued rejuvenation by hybridization and promulgated what afterward came to be known as the Knight-Darwin law. This was stated by Darwin in the aphorism, "Nature abhors perpetual selffertilization." Our views on this subject, as will be shown in the next chapter, must now be considerably modified; nevertheless Knight partially outlined a great truth.

One other plant breeder must be mentioned as markedly in advance of his age. The elder Vilmorin in the middle of the nineteenth century established a principle which has not until recently been given the credit it deserves. Vilmorin insisted that the only method of improving plants by selection is to judge each plant by the average condition of its progeny. This is Vilmorin's isolation principle and 
is the direct ancestor of Johannsen's genotype conception of heredity.

Other items of fact and improvements upon technique were numerous then as they are today, but these few discoveries comprise the only notable contributions of the hybridizers until Abbot Mendel's epoch-making researches on the pea (Pisum sativum) in the little cloister garden at Brünn.

The elements of Mendelianism as they apply to animals have already been discussed. In taking up some of the important neo-Mendelian facts as they are found in the higher plants, I propose to describe types of Mendelian inheritance with the idea of showing how facts that are apparently non-related may be included under one descriptive notation. The illustrative material is drawn largely from my own pedigree cultures of maize or Indian corn because they are naturally more familiar, but the points discussed by no means apply only to corn.

It is of no small importance that strict Mendelian notation has been found to apply to the facts of inheritance in both plants and animals. Sex has arisen separately in both kingdoms, and probably even more than once in the regetable kingdom. It is therefore a great argument for the universality of Mendelian inheritance in sexual reproduction that the general facts are so similar in both types of organisms. If many facts in each kingdom can be included in the Mendelian conception of heredity, the probability that it is a truth of universal scope increases by geometrical progression. Let us see whether or not this is true.

Let us examine first a simple case of monohybridism, i.e., a case where the parents differ by but one character. Such a character distinguishes sweet corn from starchy corn. 
The wrinkled condition of the seeds of sweet corn results from an inability to mature starch grains. The starchy corns, as the name indicates, possess this feature. One may say, therefore, that the germ cells of starchy and of

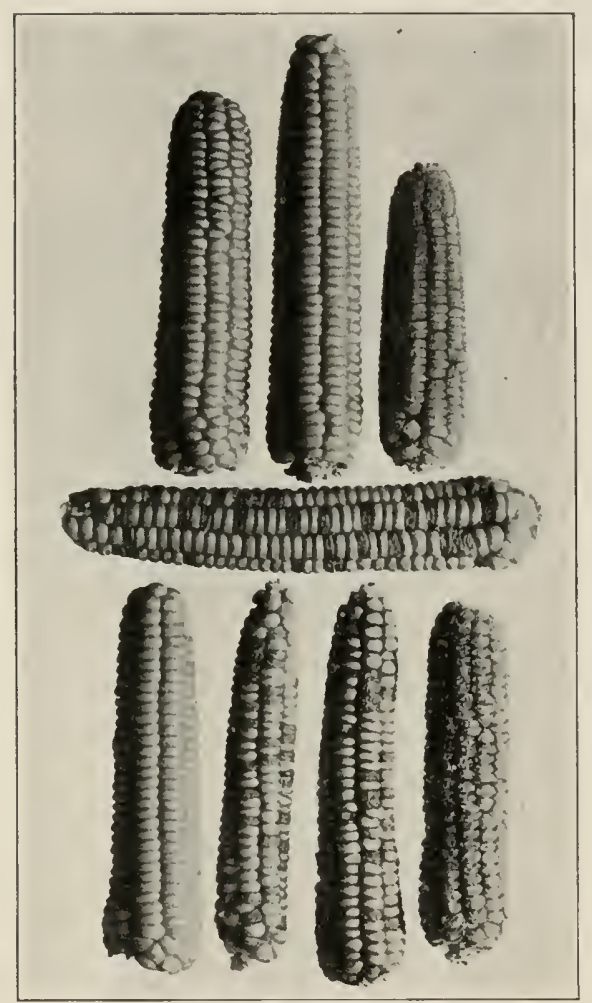

Fig. 39.-Segregation of starchiness and non-starchiness in maize. Above parents and $F_{I}$ generation, in center $F_{2}$ generation, below $\mathrm{F}_{3}$ generation. sweet corns are differentiated by the presence and the absence, respectively, of the ability to transmit starchiness (Fig. 39).

In the higher plants as in animals the gantetes or germ cells are simplex in character. Two germ cells, one male (from the pollen) and one female (in the ovule), fuse to form the duplex cell or zygote that develops into the plant. The zygote is the new plant generation of which the seed (except the seed coat) is but a resting stage of its development. Starchiness and nonstarchiness (sweetness) are seed characters. One can see just what seed characters the plant possesses, therefore, as soon as the seed is fully developed. When two gametes both carrying the factor starchiness $(S)$ produce a 
seed, that seed breeds true to the character starchiness. When two gametes both lacking the factor starchiness $(s)$ produce a seed, that seed breeds true to the character non-starchiness or sweetness. The production of gametes and zygotes may be illustrated thus:
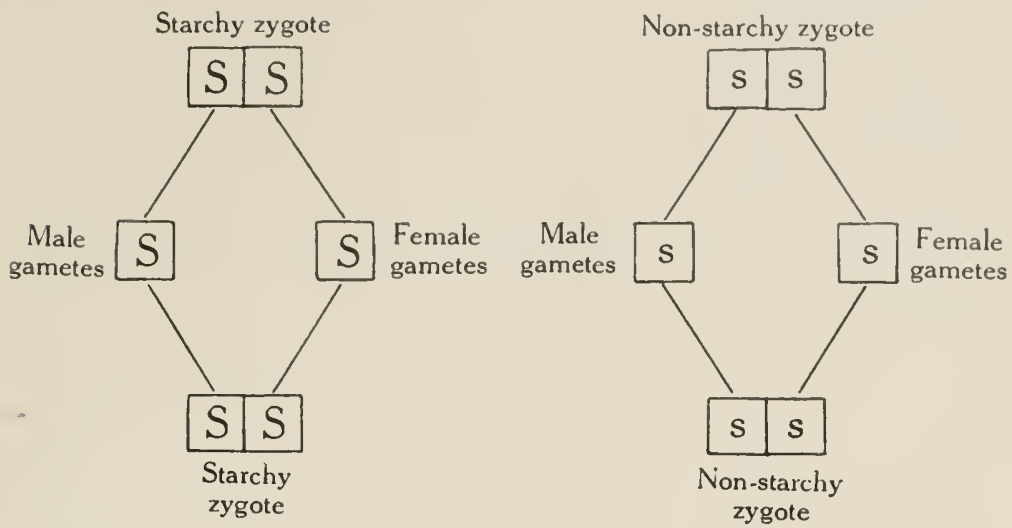

When a gamete from a starchy strain fuses with a gamete from a non-starchy strain a hybrid zygote is formed. This zygote is starchy like the starchy parent because the presence of the starchy factor from one parent is sufficient to cause the development of starch. But the gametes, both male and female, formed by this hybrid or heterozygote, are not alike. Half of them contain the starchy factor and half of them are without it. This being true, when the male and female gametes of the hybrid pair indiscriminately, the following combinations occur in equal numbers:

$$
\text { SS : Ss : sS : } \text { ss. }
$$

The first term of this ratio, resulting from the fusion of two starchy gametes, is a starchy zygote that breeds true; the second and third terms, resulting from the fusion of a starchy and a non-starchy gamete, are again hybrid in 
character; while the last term, resulting from the fusion of two non-starchy gametes, is a zygote that breeds true to non-starchiness. Since the zygotes having the gametic constitution shown by the first three terms are alike in looks, the second hybrid generation ( $\mathrm{F}_{2}$ generation) gives approximately three starchy zygotes to one non-starchy zygote. But the three starchy zygotes, though alike in looks, are different in their breeding capacity. One out of three breeds true to starchiness; two out of three are hybrid and again segregate into starchy and non-starchy in the next generation. Diagrammatically the process may be illustrated thus:

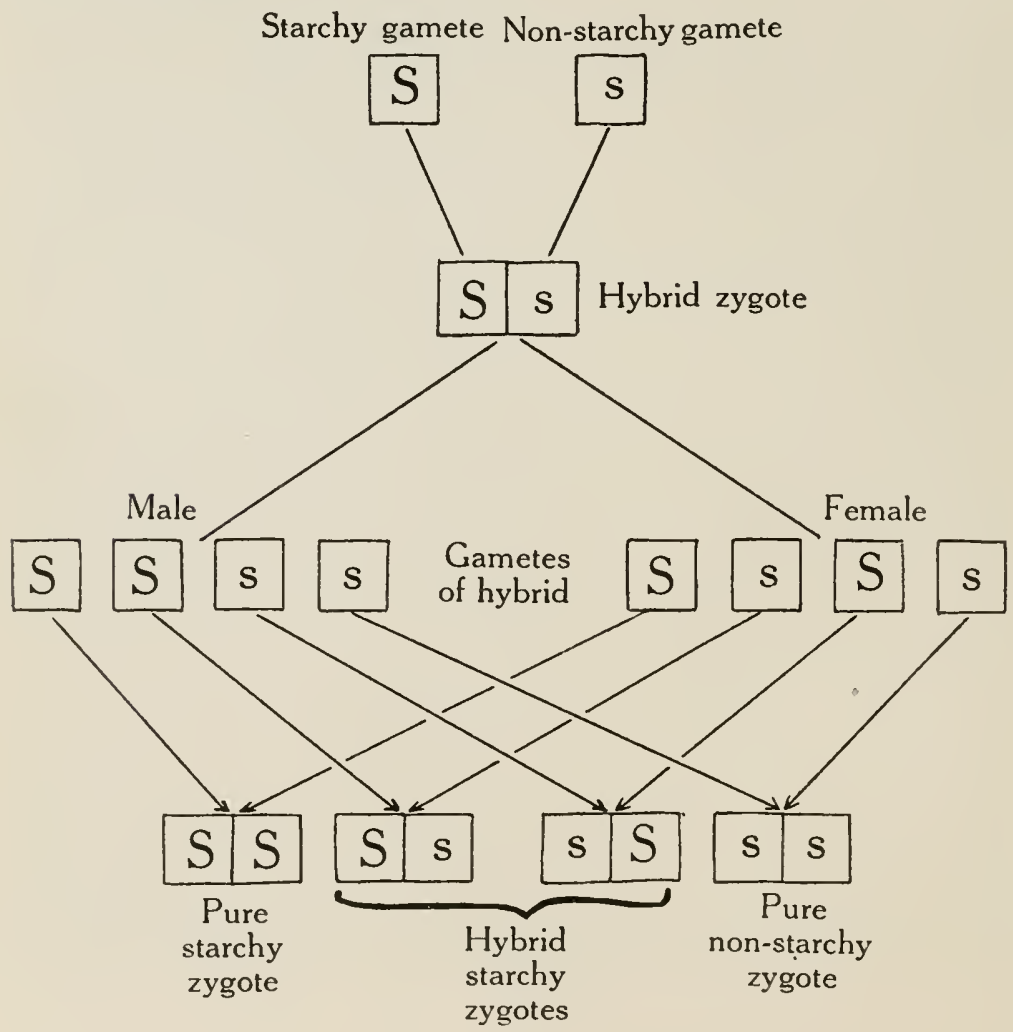


In general one may say that when two individuals are crossed which differ from each other in paired characters, of which one can usually be interpreted as the absence of the other, these parental characters reappear in the second hybrid generation unchanged in vigor and in potency. The first hybrid generation may be exactly like one parent in any particular character, in which case that character is completely dominant; or, the hybrid may be intermediate between the two parents in that character, in which case dominance is incomplete or absent. Of these two phenomena the behavior of the second hybrid generation is by far the most important. The parental characters are reproduced in it in constant ratios; and it is believed that this could only come about if the factors that represent these characters in the germ cells of the hybrid occur there unchanged and in equal numbers. Generalize this viewpoint and we have the belief that organisms behave in heredity as if they were mosaics of character units, each unit being represented in the germ cell by one or more factors of unknown nature. These factors are commonly transmitted independently of one another, and upon them depend the characters of the individuals to which they give rise.

Stated in fewer words, the essential feature of Mendelianism is the segregation of potential characters in the gamete in a state of apparent purity, and their recombination by the law of chance through random mating. The term "Mendelian notation" was therefore used advisedly. Mendelian notation is a simple interpretation of certain facts of heredity obtained in pedigree cultures. It is a convenient notation and is used much as the element symbols are used in chemistry. It makes no difference to analytical 
chemistry whether or not an atom is a reality, for the law of "Definite and Multiple Proportions" upon which analytical chemistry is based is still valid. In the same way, it makes no difference whether one regards unitcharacters as actual units and their segregation as complete, or whether one sees in organisms a mutual dependence between characters and a quantitative or partial segregation among gametic factors, the notation is useful either way to make clear the facts of heredity as shown by actual experiment.

In a simple case such as that which has just been considered (segregation of starchy and non-starchy seeds), the mathematical reasoning that led Mendel to his conclusions can be shown to be correct. In dealing with some 30,000 progeny, I have found that in the $F_{2}$ generation there were 23,529 starchy and 7,8 I I non-starchy seeds, a ratio of 3.003 I : $0.9969 \pm .0066$. This is a $3:$ I ratio well within the probable error. Furthermore, I find that the positive and negative departures from the 3 : I ratio that occur in individual ears, are exactly what should be expected by the mathematical theory of error.

In more complicated cases, sometimes there are departures from the ratio expected normally that can hardly be explained by the theory of error, yet in such cases one is warranted in assuming that misunderstood complications either obscure or modify the action of Mendel's law.

Since such accurate ratios as that given above cannot be expected except by absolute division of the gametes into two kinds equal in number, it is difficult to believe that there is not real, exact, and complete segregation. This would be the inevitable conclusion but for the fact that extracted pure types do not always breed true as they 
should. An example of this kind has arisen in connection with the character under discussion. Among many thousand non-starchy seeds that breed true after having been extracted from a cross, one occasionally appears which is semi-starchy and transmits this character. What is the explanation of this phenomenon? The aberrant individuals are too few to support any theory of partial segregation yet devised. Is it not more logical to believe that the dominant character has been formed anew? It may be asked why, if a new character is formed, does it happen to be the character with which we were previously dealing rather than some other character? It might be answered that other characters do sometimes arise. I believe, however, that a more satisfactory answer can be given. Protoplasm undoubtedly differs chemically in distinct species, and various chemical compounds have individual tendencies toward certain specific reactions. Each species of organism may therefore have certain paths of least resistance along which variations tend to go. In maize one path appears to lead to the production of starch (Fig. 40).

Many characters in the higher plants have been shown to be inherited as simple monohybrids. Color has been a favorite subject and its transmission in over thirty species has been more or less clearly determined. The list of structural characters investigated is also large. It includes such different things as tallness and dwarfness (peas and beans), hairiness and glabrousness (various species), beardless and bearded ears (wheat), much-serrated and littleserrated leaves (nettles), two-celled fruit and many-celled fruit (tomatoes). A criticism has sometimes been raised that most of these characters are morphologically unimportant. In a measure this criticism is valid, yet there is 
a good reason for its truth. The mechanism of the hereditary transmission of any character can only be demonstrated when two "crossable" varieties differ in this character. The characters most important to a species are

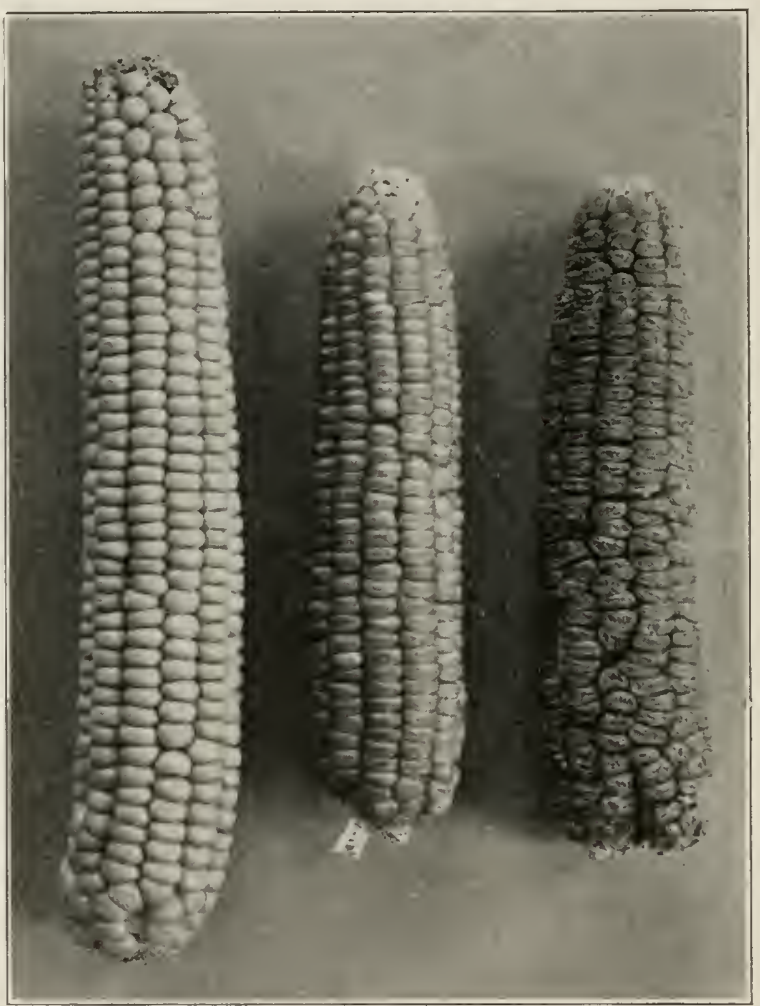

FIG. 40.- Starchiness in an extracted recessive. On either side normal segregates, in center semi-starchy ear grown from non-starchy seed.

characteristic of the species. No individual is without them; and this being true, the method of their inheritance must remain unknown. It is often impossible even to tell whether there is a single or a complex set of factors con- 
cerned in the hereditary transmission of a character. Complexity shows only when the two parents differ in several factors involving the same character. If they differ by only one factor, inheritance may appear to be very simple; but crosses between other individuals similar to the former in appearance may show an entirely different state of affairs. A very good illustration of what is meant by this statement is the inheritance of the purple color of maize that occurs in the single layer of cells immediately beneath the hull or pericarp-the aleurone cells (Fig. 4r). When an individual of a variety containing the purple color is crossed with certain white varieties, the hybrid is purple, showing dominance of the purple character. The second hybrid generation produces three purple seeds to one white seed. Since this is the normal monohybrid ratio, one would suppose that the character in question is simple. This is only apparently the case, however, for when the same purple variety is crossed with other white varieties the ratio of purple to white seeds is such that one knows the varieties must have differed by two gametic factors affecting the development of the purple character, instead of one. In order that this matter may be made clear, the dihybrid ratio must be explained.

The dihybrid ratio is that obtained when the parents differ by two character pairs. It is the algebraic product of two $(3+\mathrm{I})$ ratios. The chances are $\frac{3}{4} \times \frac{3}{4}=\frac{9}{16}$ that the two dominant or presence characters occur together, $\frac{3}{4} \times \frac{1}{4}=$ $\frac{3}{16}$ that the dominant character of the first pair meets the recessive or absence character of the second pair, $\frac{1}{4} \times \frac{3}{4}=\frac{3}{16}$ that the recessive character of the first pair meets the dominant character of the second pair, and $\frac{1}{4} \times \frac{1}{4}={ }_{1}^{1}$. that both the recessive characters occur together. If we let the 
two pairs of characters be represented by $A$ and $a$ and $B$ and $b$ the ratio of the occurrence of types in the $\mathrm{F}_{2}$ generation is $A A+2 A a+a a$ and $B B+2 B b+b b$ when considered

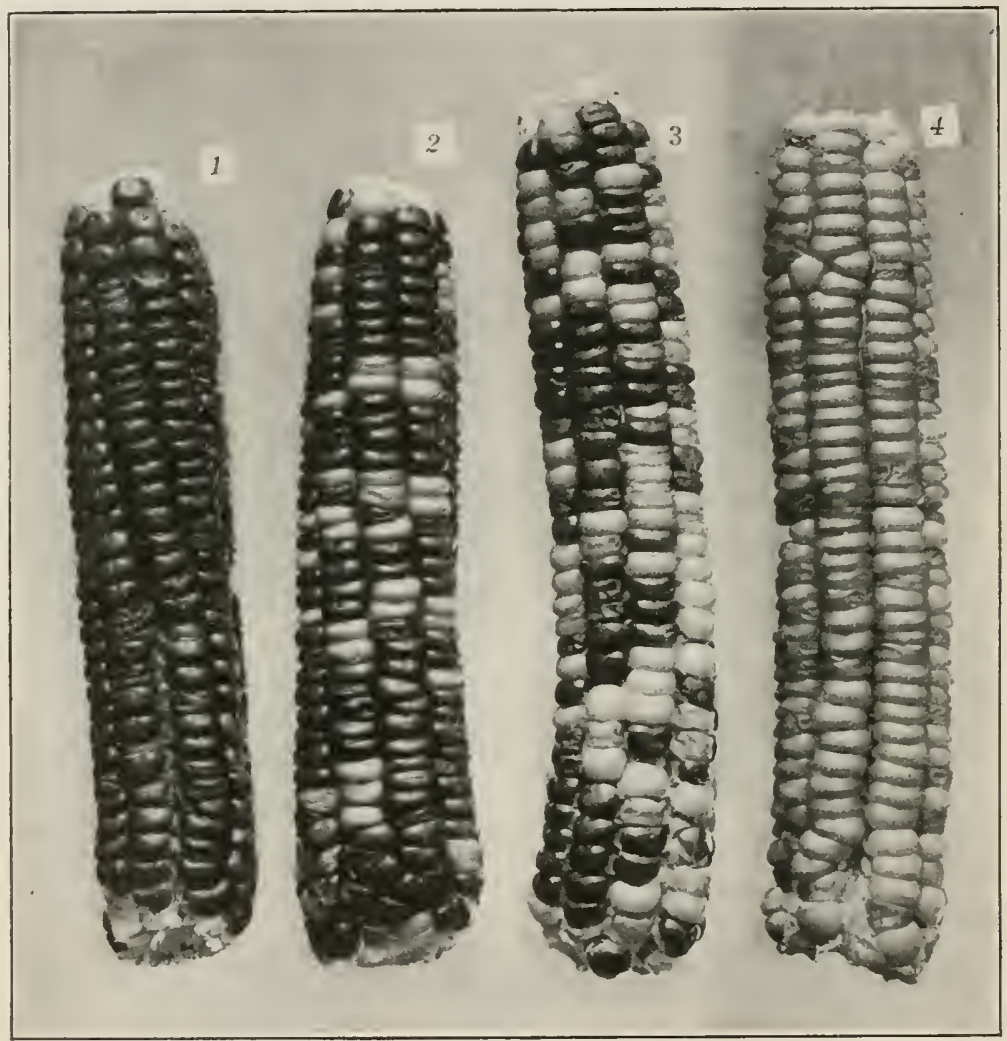

Fig. 4r.--Inheritance of purple aleurone cells. Ear I a pure extracted purple, ear 2 monohybrid with 3 to 1 ratio, ear 3 dihybrid with 9 to 7 ratio, ear 4 a pure extracted white.

separately. If one wishes to obtain the probable ratio when both characters are considered together he has but to multiply the two series together. This gives the product $A A B B+A A b b+a a B B+a a b b+2 A A B b+2 a a B b+2 A a$ 
$b b+2 A a B B+4 A a B b$. Since heterozygotes resemble homozygotes in appearance in the cases of complete dominance, however, the visible appearance of the $\mathrm{F}_{2}$ generation is $9 A B: 3 A b: 3 a B: \mathbf{1} a b$.

Perhaps the easiest method of showing the combinations of gametes in Mendelian inheritance is by the use of four squares multiplied once by four for each character pair. In the case of dihybrids sixteen squares are necessary. Write each possible gametic combination of the male cells in each horizontal row of squares, $A B, A b, a B$, and $a b$. Next write the same combinations for the female gametes in each vertical column of squares. This gives all the zygotic combinations possible.

We are now ready to see just why one cannot tell whether he is dealing with a simple or complex state of affairs in Mendelian inheritance. In the monohybrid ratio of purple and non-purple aleurone cells of maize the segregation in the $\mathrm{F}_{2}$ generation is:

\begin{tabular}{|l|l|}
$P$ & $p$ \\
$P$ & $P$ \\
\hline$P$ & $p$ \\
\hline$p$ & $p$ \\
\hline
\end{tabular}

Three purples to one non-purple are produced.

In the dihybrid ratio when other white varieties were used as one of the parents, nine purples to seven non-purples were produced. This simply means that two factors are necessary to produce the purple color. These factors may 
be represented by the letters $C$ and $P$. The gametic formula of the purple variety is $C P$; the gametic formula of these particular white varieties is $c p$. If either $C$ or $P$ is absent from the zygotic formula of $\mathrm{F}_{2}$ then the zygote is white as is shown in the diagram.

DIAGRAM ILLUSTRATING INHERITANCE OF PURPLE ALEURONE

CELLS IN MAIZE WHEN TIY FACTORS ARE CONCERNED

\begin{tabular}{|c|c|c|c|}
\hline$C P$ & $C P$ & $C P$ & $C P$ \\
\hline$C P$ & $C p$ & $c P$ & $c p$ \\
\hline Purple & Purple & Purple & Purple \\
\hline$C p$ & $C p$ & $C p$ & $C p$ \\
\hline$C P$ & $C p$ & $c P$ & $c p$ \\
\hline Purple & White & Purple & White \\
\hline$c P$ & $c P$ & $c P$ & $c P$ \\
\hline$C P$ & $C p$ & $c P$ & $c p$ \\
\hline Purple & Purple & White & White \\
\hline$c p$ & $c p$ & $c p$ & $c p$ \\
\hline$C P$ & $C p$ & $c P$ & $c p$ \\
\hline Purple & White & White & White \\
\hline
\end{tabular}

The formula of the purple is therefore $C C P P$, but whites with formulas $C C p p, c c P P$, and $c c p p$ may exist. When the whites have formulae $C C p p$ or $c c P P$ they give monohybrid ratios when crossed with the purple, but when they have the formula $c c p p$ a dihybrid results.

This illustration shows both why one cannot tell just how complex a character is, and why characters essential to the species cannot be analyzed.

These cases of Mendelian inheritance are types. Other 
cases may be more complex in appearance but this is a surface complexity only. They yield to similar analyses when they are properly understood. This complexity in appearance has many times made cases appear to be exceptions to Mendel's general law. Various ratios have been obtained that were seemingly inexplicable, but these one by one have been found to be merely variations of the simple ratios that we have just discussed. Perhaps the most interesting of these aberrant ratios are those cases of heredity dealing with latent characters. We will consider some of these briefly as examples of the various manifestations which are found in the hereditary transmission of plant characters.

One of the most important classes of latency we have just discussed. It is called latency of separation. Where a character exists through the interaction of two factors, these factors may at some time become separated, that is, possessed by different individuals. When this occurs the character is apparently lost and does not again appear unless two individuals bearing the complementary factors are crossed. This is the explanation of the old phenomenon of reversion after crossing. It was first explained by Bateson from experiments with sweet peas. He found that two white varieties yielded the purple color of the wild sweet pea when crossed. In the same way I have found that the white seeds in the dihybrid ratio of $9: 7$, shown by the purple $X$ non-purple cross of maize varieties, give purple seeds when crossed at random. The non-purples exist in the following ratios:

$$
7\left\{\begin{array}{lllll}
\mathrm{I} & C & c & p & p \\
2 & C & c & p & p \\
\mathrm{I} & c & c & P & P \\
2 & c & c & P & p \\
\mathrm{I} & c & c & p & p
\end{array}\right.
$$


When crossed at random, there are $7 \times 6=42$ possible combinations of which 24 give all white seeds and I 8 give some purple seeds. Of these i 8 ears there would be on the average 2 pure purples, 8 with purples and whites in a ratio of I : $\mathrm{I}$, and 8 with purples and whites in a ratio of $\mathrm{I}: 3$. Such results have been obtained experimentally and examples are shown in Fig. 42.

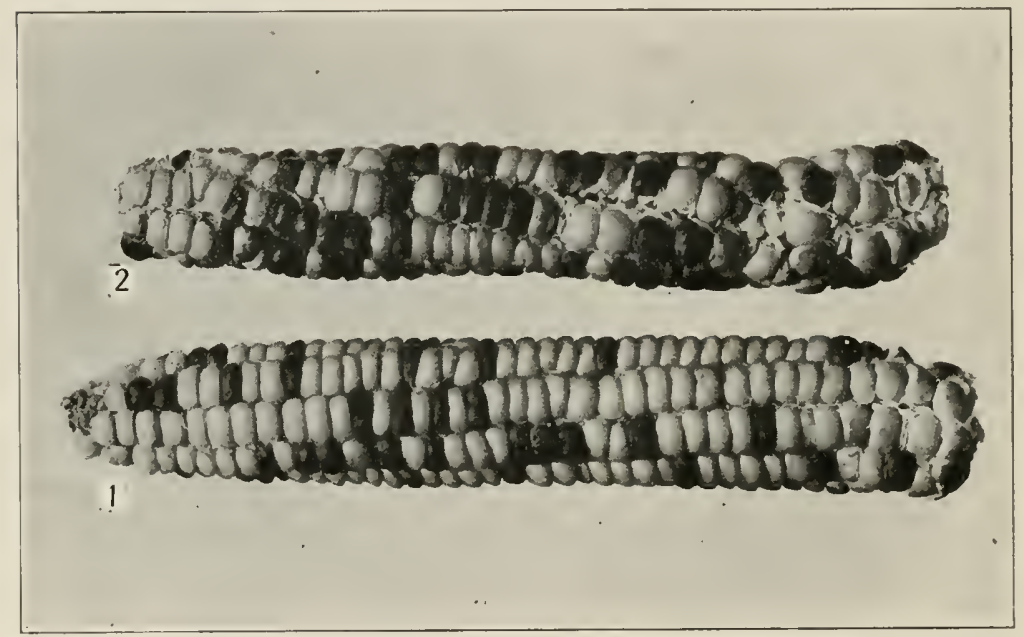

FIG. 42.- Reversion due to latency of separation. Purple seeds produced by crossing whites. Ear I, I to 3 ratio; ear 2, I to I ratio.

A different kind of latency is simply invisibility of a character due to some obscuring factor which may be removed by means of a cross in which the latter is absent. This latency is called that of hypostasis, a hypostatic character being one which is obscured by another called the epistatic character. There are instances where this epistatic character may be removed mechanically and the hypostatic character revealed. Such a one is the red-eared maize, for the color there lies in the hull or pericarp. This 
may be scraped off to show the color of the true seed which may be either yellow or white. In most cases, however, the relations are physiological and the hypostatic character can be demonstrated only by crossing. For example, the maize with purple aleurone cells carries also a factor for red aleurone cells which can be demonstrated only by crossing it with a variety in which the purple factor is absent. There is produced an $\mathrm{F}_{2}$ generation with a ratio of 27 purples : 9 reds : 28 whites.

This statement may appear to be a direct contradiction of the interpretation of the inheritance of purple aleurone color that has just been given. In reality it is merely another illustration of the fact that one can never know definitely the factors involved in producing a character, for he can never feel assured that tre parents involved in the cross have differed in all the factors that affect its development. When the purple was crossed with the white and a ratio of 9 purples : 7 whites obtained in the $\mathrm{F}_{2}$ generation, it was proper to interpret the purple parent as $P P C C$ and the non-purple parent as $p p c c$. But when the purple is crossed with another non-purple and a ratio of 27 purples: 9 reds : 28 whites is obtained in the $\mathrm{F}_{2}$ generation, it is clear that a different interpretation is necessary. The purple parent has the formula $P P R R C C$ and the white parent the formula $p p r r c c$. The zygotic formula of a pure red seed is $R R C C$ and of a pure purple seed is $P P R R C C$, but a seed with the formula $P P C C$ is white. In other words, the factor $P$ produces the visible purple color only in the presence of factors $C$ and $R$. The purple has always the constitution $P P R R C C$; when it is crossed with whites having the characters $P P R R c c$ or $P P r C C$, a ratio of 3 purples : I white is obtained in $\mathrm{F}_{2}$; when it is crossed 
with a white having the composition $P \operatorname{Pr} c c$, a ratio of 9 purples : 7 whites is obtained in $\mathrm{F}_{2}$; when it is crossed with a white having the composition p prrcc, a ratio of 27 purples : 9 reds : $2 S$ whites is obtained in $F_{2}$.

It is possible that another factorial difference may yet be found which will show this character to be still more complex. Baur, Bateson, Saunders, and Gregory have shown that the sap colors of the flowers of Antirrinum, of Lathyrus, of Matthiola, and of Primula belong to this type and are yet more complex. But this does not affect our general conception of the inheritance of the colors in the least.

Sometimes latent characters very similar to this are due . to the presence of a second inherited factor which does not allow the first character to develop. This is calied latency due to inhibition. Similarly there may be inherited characters which help to a more perfect development other independently transmitted characters. This is undoubtedly an important phase of Mendelianism for although we may conceive factors as holding within themselves the potentialities of certain characters, they undoubtedly are influenced in their development by the development of other inherited characters. One may imagine that factors representing characters may be transmitted but are either not expressed at all or are developed to a limited degree owing to the presence or the absence of other inherited characters that affect this development.

Perhaps this theoretical conception may be made plain by an example of what has been termed latency of fluctuation. In this class are included characters which are potentially present in an organism but which may develop to a greater or less extent due to varying influences which sur- 
round them. There is a red color in the pericarp or hull of the maize seed which is transmitted as a simple Mendelian monohybrid when crossed with a variety in which the character is absent, but the color develops to its full extent only in bright sunlight. When a black bag is placed over the young ear the color does not develop at all, yet such a colorless ear will transmit the color as well as if that ear had developed in bright sunlight. One may easily see then how he might be deceived in the classification of such individuals and thereby draw wrong conclusions regarding the inheritance of the character.

Though the example just given is one of latency of fluctuation, it really consists in the character being partially inhibited by absence of light. One may see from it, however, that there can be real latency of inhibition through the influence of inherited factors that affect the development of independent characters.

We have hitherto considered characters that seem to be transmitted independently of one another. Let us now spend a moment in discussing characters that appear to be either coupled or antagonistic to each other in their transmission. Some very interesting results on this subject have been obtained by Emerson (Fig. 43). There are maize varieties which are red in both the pericarp of the seed and the cob. When one of these is crossed with a variety in which color is absent in both the pericarp and cob, the second hybrid generation produces three ears like the colored parent to one ear like the white parent. One might suppose that the color in pericarp and in cob was due to a single gametic factor. This can hardly be the case, however, for there are varieties with red pericarp and white cob and varieties with white pericarp and red cob. When two 
such varieties are crossed together the first hybrid generation produces ears with red pericarp and red cob like the colored variety in the previous cross. In the second hybrid generation, however, we find produced I red

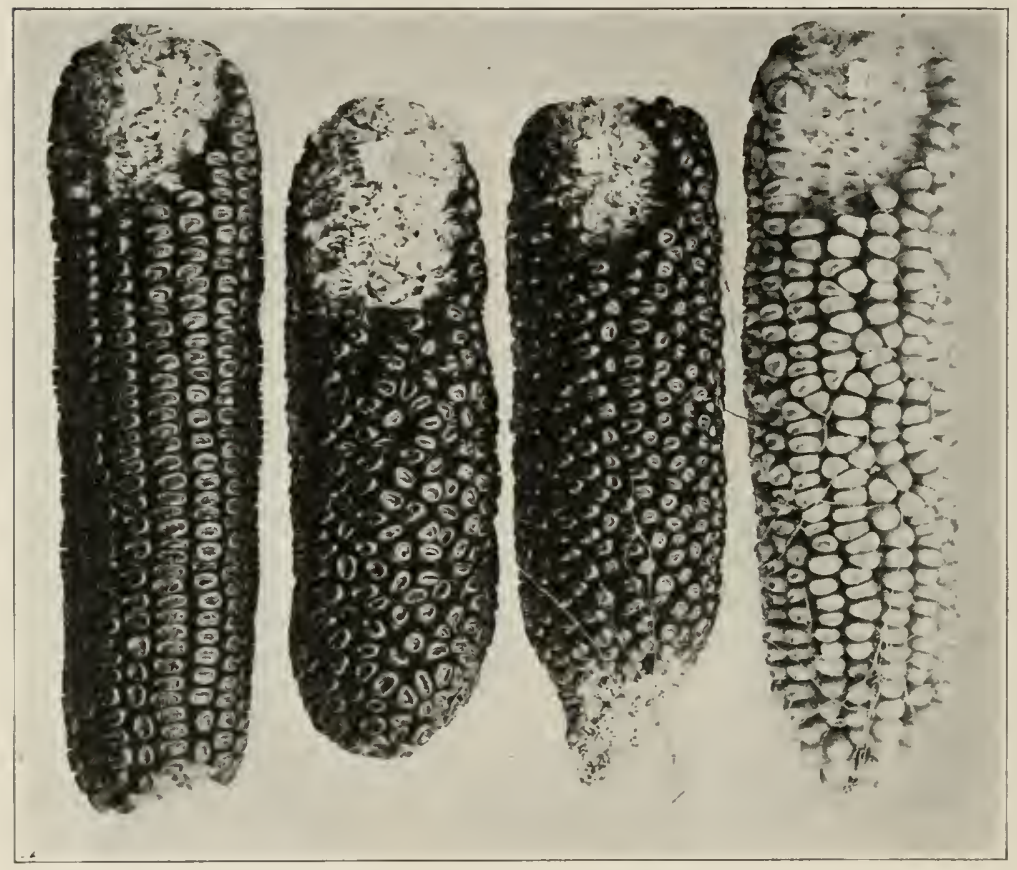

FIG. 43.- Red pericarp and red cob coupled in inheritance. (Photo by Emerson.) $\mathrm{F}_{2}$ generation shown. Ears $\mathrm{I}, 2,3$ with red cob and red pericarp, ear + with white cob and white pericarp.

pericarp with white cob: 2 red pericarp with red cob: I white pericarp with red cob. The first and third classes breed true to their conditions, but the second class proves to be always heterozygous and breaks up in the next generation as did those with similar characters of the first hybrid generation. 
Without going into the theory of this phenomenon it is apparent that in the first case red pericarp and red cob

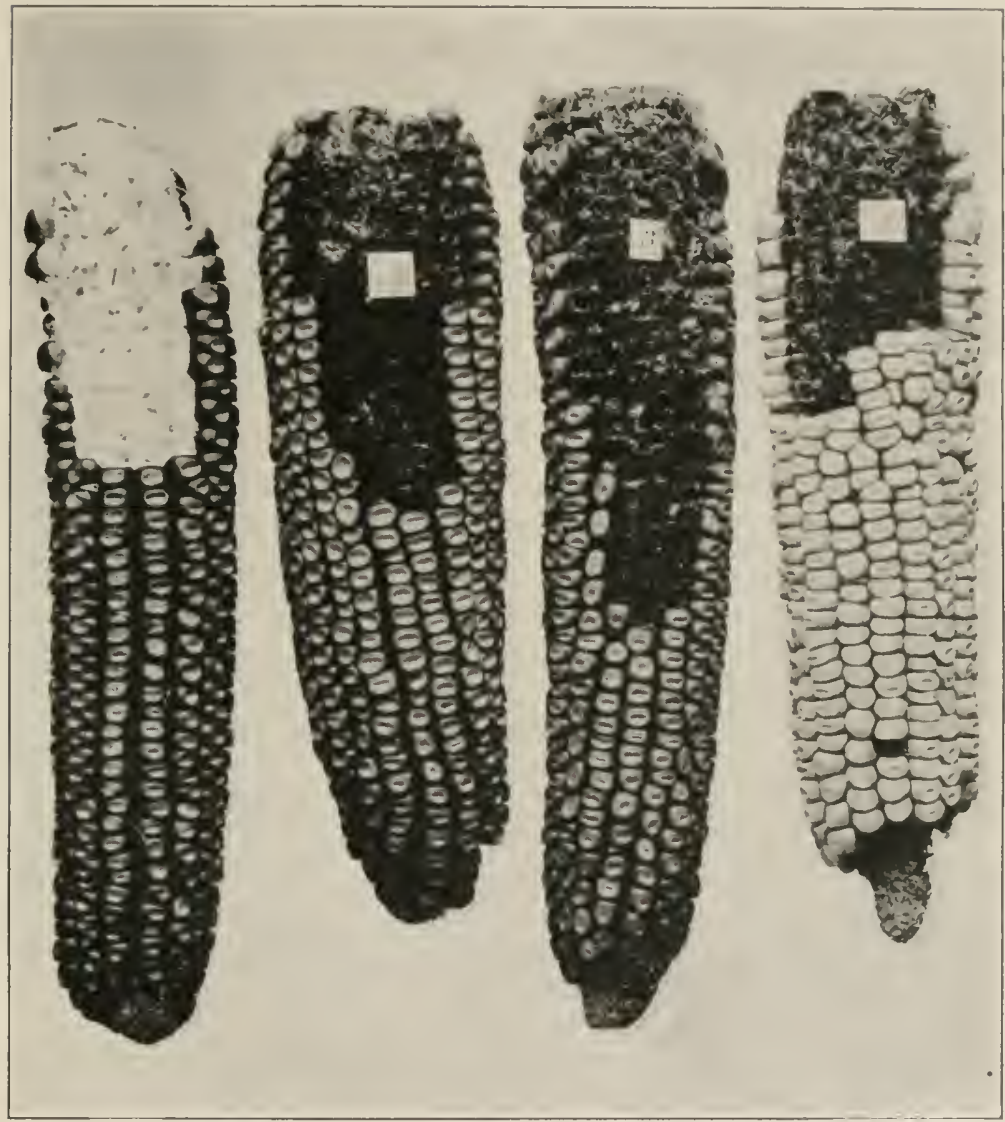

FIG. 44-- Red cob and red pericarp antagonistic in inheritance. (Photo by Emerson.) $\mathrm{F}_{2}$ generation shown. Lars $\mathrm{I}$ and 4 , like parents, are pure types. Ears 2 and 3 are heterozygous.

were not produced by a single factor in the usual sense, but by two individual factors coupled in their inheritance. In the second cross the two factors were not coupled; in fact 
they were just the opposite, they were antagonistic to each other so that no pure types were produced having red pericarps and red cobs (Fig. 44). This is an example of a feature which is probably very widespread in the plant world, but of which we at present know little. It is cited for two reasons; first, to show that characters may at one time be antagonistic to each other and at another time coupled together, and second, to show that one is not able to say beforehand whether a manifestation of a character in several organs is due to one or to several separately inherited factors.

Leaving out of consideration sex-limited inheritance of which little is known in plants, we have now briefly gone over simple type cases of some of the most important presentday Mendelian knowledge; but we have considered only crosses in which the potential character or characters are present in one parent and absent in the other. At least they behave that way and may reasonably be so interpreted. Such differences between parents are qualitative, but most differences between parents are quantitative and give an apparent blend in the first hybrid generation. Nearly all cases where varieties differ in the size of their organs are of this kind. Can such phenomena be interpreted by Mendelian notation? I believe they can. One may think of a factor for a character being present in the germ cell not only once but twice or even a greater number of times. If these factors are transmitted independently and are not paired with each other, but each with its own absence, one may very easily interpret size inheritance. For example, when a certain dent variety of maize is crossed with a flint variety as shown in Fig. 45, an intermediate condition is obtained in the first hybrid generation. In the second 
hybrid generation one ear like each original parent is obtained out of every sixteen instead of every four (Figs. $46,47)$. This inheritance is therefore dihybrid in character. In like manner, a higher number of transmissible factors may affect the development of what is to the eye a single character.

Since dominance is not an essential feature of Mendelianism, size characters may show intermediates or blends in the first hybrid generation and still fulfil the essential conditions of Mendel's law by recombining in such a fashion as to produce individuals like either parent in the second hybrid generation provided a sufficiently large number of individuals to allow for the recombination of several factors is grown in that generation. Such recombinations do occur and can be shown by experiment. For example, the small variety of corn, Tom Thumb, when crossed with a larger variety like the Black Mexican (Fig. 48 ), gives a first hybrid generation that is intermediate between the two parents. One may call this a blended condition; yet if there were blended inheritance this condition would be transmitted, while if Mendelian recombination occurred, sizes comparable to either parent would be obtained in the second hybrid generation. Such extremes were obtained as is shown in the figure.

If the possible Mendelian interpretation of quantitative characters has been made clear, the statement that Mendel's law is probably universally applicable where sexual reproduction occurs will not seem rash. There are still some apparent exceptions to the law, but they are so few that one may well believe we simply do not know how to bring them into line and not that they are actual exceptions. Of course it is quite likely that there are other laws which 
modify the action of Mendel's law in a manner similar to that in which the physical laws holding good under theoretical conditions are modified under conditions existing in nature.

Granted, then, that Mendelianism is broad in scope, could it have been of great value during the progress of

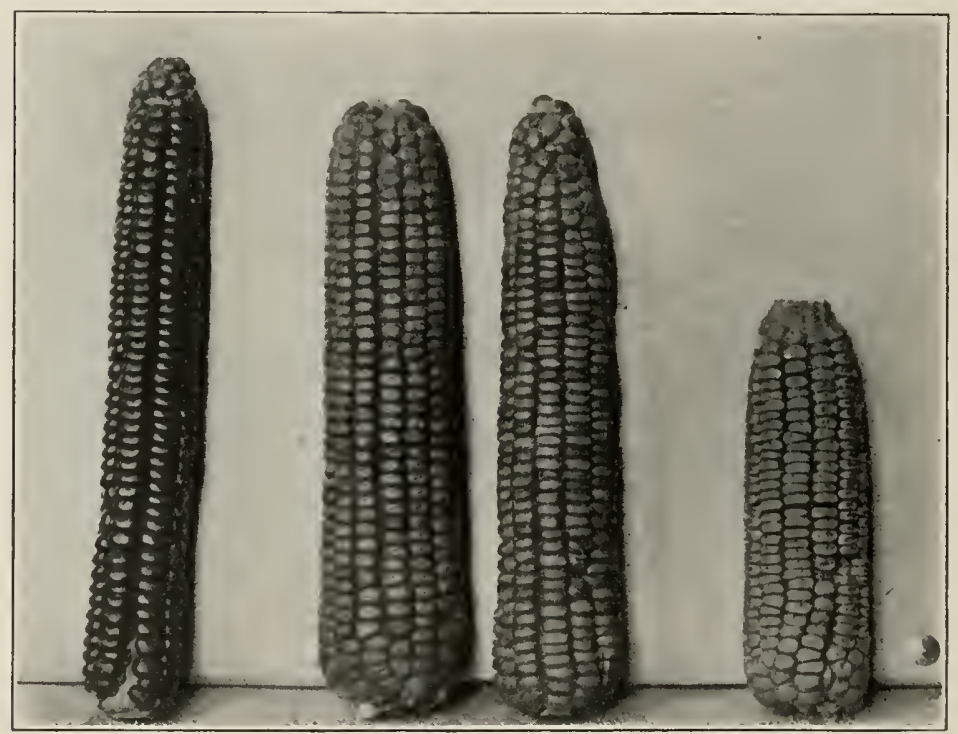

FIG. 45.-Inheritance of physical condition of endosperm in maize. Parents and $F_{I}$ generation shown.

evolution? That it should have been of great value to very primitive forms of life does not seem possible, and even after the origin of sex its precise importance is somewhat problematical. It has disposed of one of the main criticisms against Darwin, at least, that of the swamping effects of intercrossing upon newly arisen characters that are an advantage to the species. Since characters are segregated as units 


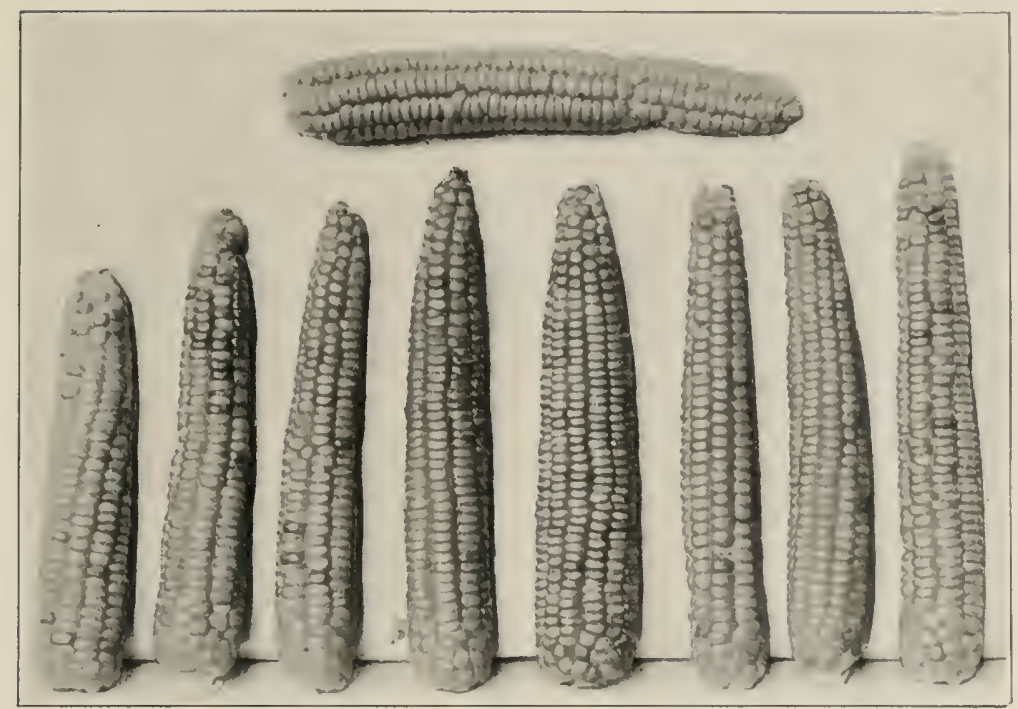

FIG. 46.--Inheritance of physical condition of endosperm in maize. Flintlike $F_{2}$ segregate above and $F_{3}$ progeny below.

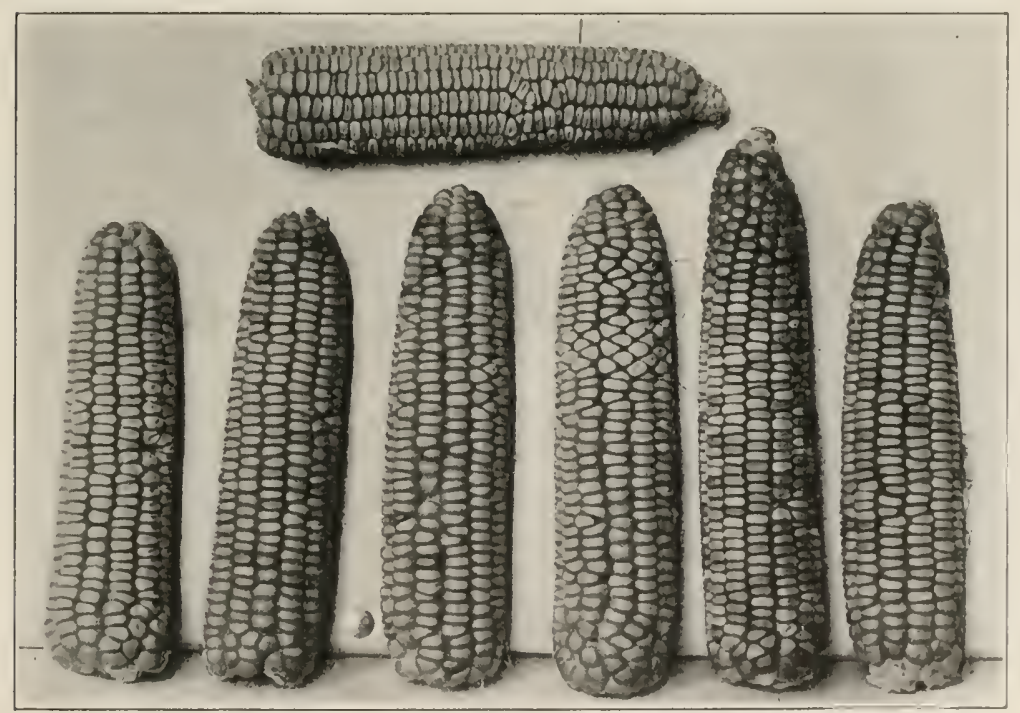

FIG. 47.--Inheritance of physical condition of endosperm in mare. Dentlike $\mathrm{F}_{2}$ segregate above and $\mathrm{F}_{3}$ progeny below. 
in the hybrid it matters not whether they are dominant or recessive or whether there is no dominance, there will be no dilution from intercrossing. A second and more important advantage, due to the operation of the law, results from the recombination of characters. Characters may be transmitted as units and chance recombinations of these characters may occur without anything really new to the organism being formed, yet in this recombination the organism as a whole may be better fitted for its environment than ever before. And this is giving recombination its smallest value, for, however independently potential characters may be transmitted, no one believes that a developing organism is simply a mass of independently developing unit-characters. The characters of an organism are more or less dependent upon one another in their expression, and in this interdependent development the greatest possibility for good recombinations occurs. Theoretically nothing new may result from the mere act of crossing, but practically a new combination of old characters may result in something quite different. A little thought and the use of chemical analogies where the same mixtures produce different chemical results under different physical conditions, show the importance of this conception to Mendelian theory. Yet it is not necessary to believe the current teaching that one has only new recombinations and not new characters to deal with in crosses. The theory that several germ-cell factors may be due to produce the same character gives us a reasonable and orthodox explanation of the origin of characters really and truly new by the interaction of gametic factors that are old. For example, let us suppose that in a certain species with a petioled leaf there is a variety which has the presence of factor $A$ pro- 


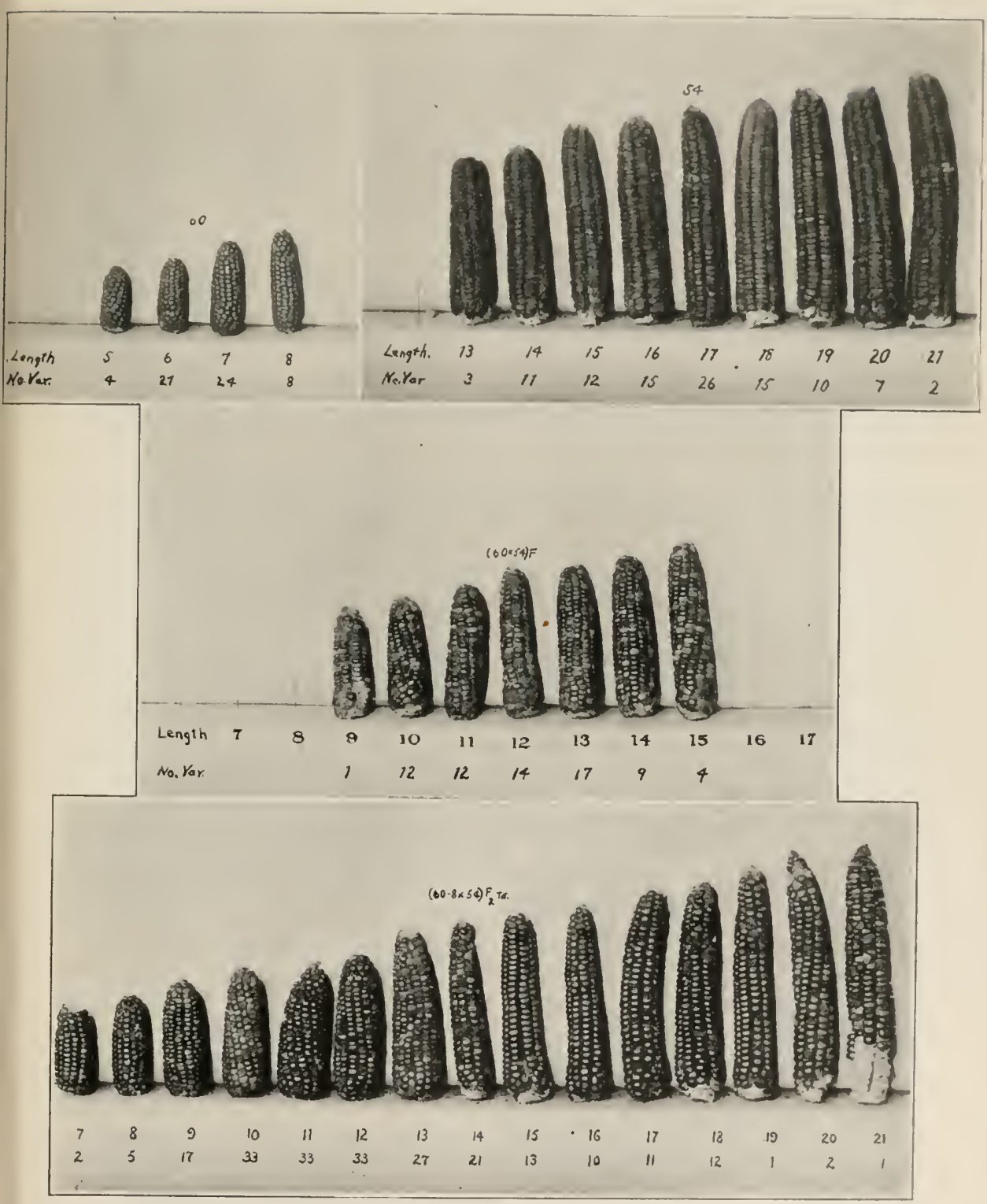

Fig. 48.-Inheritance of length of ear in maize. Parents above, $F^{\mathrm{x}}$ generation in center, $F_{2}$ generation below. Class sizes in centimeters with number of variates below. 
ducing a slight tendency toward a sessile leaf. Factors $B$ and $C$ may ultimately arise as modifications of factor $A$. Each alone may produce the same result as $A$; yet if they are transmitted independently, are not allelomorphic to each other and are cumulative in their action, a sessile leafa new character-is produced.

The remainder of this chapter will be devoted to a consideration of Johannsen's genotype conception of heredity. It may seem as if Mendelism has been dropped abruptly to take up a new subject. This is not the case. The genotype conception of heredity is merely an acknowledgment of the universality of Mendelism.

Johannsen, who developed the genotype idea, found that some variations were not inherited. These were the general variations in relative perfection of development of parts, caused by varying physical and chemical conditions of environment. This is only an acceptance of Weismann's theory that inheritance is from germ cell to germ cell, and that ordinary environmental influences affecting the body only are not transmissible. Of course both Weismann and Johannsen acknowledge that certain changes in environment may produce structural modification of the germ plasm and therefore a heritable variation, but whether the heritable variation produced is ever identical with the adaptive response of the parent organism is still in dispute.

If this contention be true, it follows that the hereditary characters of an organism are determined by the constitution of the fertilized egg from which it came. Johannsen denotes the sum total of the gametic factors making up a zygote by the word genotype. If two individuals possess identical gametic factors, they are members of the same genotype. Of course no one can describe a genotype in 
concrete terms. It is a theoretical, a philosophical, concep)tion. If one crosses two individuals, the genes or gametic factors common to each breed true. He can obtain an idea of the behavior in heredity of those factors only which are not common to the two parents. These factors segregate and recombine in definite proportions. 'They follow Mendel's law. The genotype conception of heredity is therefore the conception that duplex or homozygous gametic factors are due to produce identical results within the limits of variability imposed by external conditions and by the influence of other independent gametic factors during ontogeny, no matter what is the appearance of the individual from which they were derived. This is a strict Mendelian conception of heredity extended to the organism as a whole.

We need not go into the many lines of work that support the genotype theory. Considered in a broad way I believe no reputable modern work is irreconcilable to it, although some authors do not so interpret their work. All modern plant breeding is in its support, for the principle of Vilmorin, the progeny test, which is the basis of all modern selection work, is founded upon the same conception. In naturally inbred plants, one has commercial strains which are mechanical mixtures of near-homozygotes, and can be immediately isolated. In naturally cross-bred plants or in bisexual animals, one has physiological mixtures, that is, hybrids or heterozygotes, from which it takes somewhat longer to isolate particular strains that are genotypically homozygous in respect to certain characters, but in which the separation is accomplished by the same means-the breeding test.

In the theoretical homozygous genotype transmissible variations may occur, for no one believes protoplasm 
unchanging. Such a conception would be mechanical and not biological. These changes must affect the germ cell structurally to be inherited. They may be large, they may be small. We may call them mutations with DeVries, or we may use the simpler and broader term, inherited variations. When they occur, new varieties may be isolated in which they are present, and these may be considered to be relatively permanent as compared with the noninherited fluctuations that continually occur due to varying environment.

One may question the stability of unit-characters as does Castle, but I cannot see how this affects the truth of the genotype conception as a help toward an idea of the process of heredity. Stability is a relative thing. Why is there not a scale of stability in biology even as in chemistry? Many unit-characters are high in the scale of stability, others may be low. Certain characters ordinarily transmitted perfectly may possibly be modifiable by selection. We might imagine their factors to be huge chemical molecules, stable as a whole but modifiable by isomerism or even the dropping off or adding on of unimportant radicles. This is a smaller issue, unimportant when compared with the genotype conception as a whole. The important point as the foundation of the modern view of heredity I give in Johannsen's own words: "Personal qualities are the reactions of the gametes joining to form a zygote; but the nature of the gametes is not determined by the personal qualities of the parents or ancestors in question." 


\section{CHAP'TER VI}

THE APPLICATION OF BIOLOGICAL PRINCIPLES TO PLANT BREEDING

In this chapter I shall take up the commercial application of some of the principles of plant genetics previously discussed.

The fact must again be emphasized that there are two kinds of variation:

I. Fluctuating variations, which are due solely to surrounding influences such as better position for development or varying fertility of the soil. Such variations are not inherited.

2. Inherited variations, which are due to some structural change in the reproductive cells. These variations may depend upon environmental conditions for their full development but not for their transmission.

Inherited variations possess the only value to the plant breeder, yet the work of improving plants is rendered a great deal more irksome by the presence of fluctuations and by the fact that one cannot tell the gametic constitution of a plant, that is, its breeding capacity, by its appearance. The whole problem of the plant breeder is to find, to fix, and to recombine desirable inherited variations, and to do this in spite of their tendency to be obscured by fluctuations. The methods used to accomplish these results will be taken up presently. First, attention must be called to a physiological phenomenon that may be made a tool of such high value to the plant breeder that the statement just made concerning his problem is apparently untrue. This matter 
is an exception, however-a thing apart from the usual breeding procedure.

It will be remembered that when a plant receives identical character factors from each parent, that character is homozygous and breeds true; but when the plant receives the character from only one parent, the character is heterozygous and shows segregation in the next generation. This heterozygous condition, though not fixable itself, since it always breaks up in the succeeding generation, is a valuable asset to the plant breeder if properly utilized and a distinct disadvantage if unrecognized.

The fusion of two gametes into a zygote which is known as fertilization effects two very different results: first, a union of the hereditary factors possessed by these gametes; second, a stimulation to the cell division necessary for normal development. Probably in every case where fertilization can take place at all there is a certain amount of this stimulus to development, but the fact of especial interest to plant breeders is that this stimulus is generally far greater in a hybrid or heterozygote than it is in a pure-bred or homozygous individual. The stimulus is simply toward greater and quicker cell division and affects only size and rapidity of maturity.

The tobacco genus (Nicotiana) furnishes an admirable type illustration of the stimulus due to heterozygosis because the species are generally self-fertilized under natural conditions. The stamens and the pistil are about the same length, and since the pollen is usually shed before the flower opens, self-pollination must occur unless foreign pollen is carried to the unopened bud by insects. When varieties of the common tobacco $N$. tabacum are crossed together, the first hybrid generation is nearly always from 5 to 50 
per cent taller than the arerage of the parents. The hybrids also have somewhat larger leaves than the arerage of the two parents although the number is generally intermediate. This statement is true in general for crosses between the varieties of most other species. When true species are crossed, however, the behavior of the first hybrid generation is somewhat different. Sometimes there is a great increase in vigor. $N$. tabacum $\times N$. sylestris gives hybrids that are nearly double the average height of the parents. $N$. tabacum $\times N$. alata, on the other hand, gives hybrids that are less than one-fourth the size of the smaller parent. Both of these crosses are sterile, so that the difference in behavior cannot be correlated with sterility. One can simply say that species vary in their affinity to cross with other species. All gradations are found, from those that produce full quotas of viable seed, to those where only an occasional seed is found or where the capsule simply develops without the formation of seed. And in general the additional vigor of the first hybrid generation increases with the ease of making the cross. With perfect ease of crossing the stimulus is roughly a function either of the number or of the kind of character pairs for which the individual is heterozygous.

Species naturally self-fertilized like tobacco or wheat must get along without the increased vigor due to heterozygosis. One notices the difference only when artificial crosses are made. Species which in nature are crossfertilized, however, are usually heterozygous for so many characters that one does not think of their vigor as being largely due to this cause. The fact is only brought to notice when the species is self-fertilized artificially, for this tends to isolate homozygous strains (Fig. 49). 
If, for example, a commercial variety of maize is self-

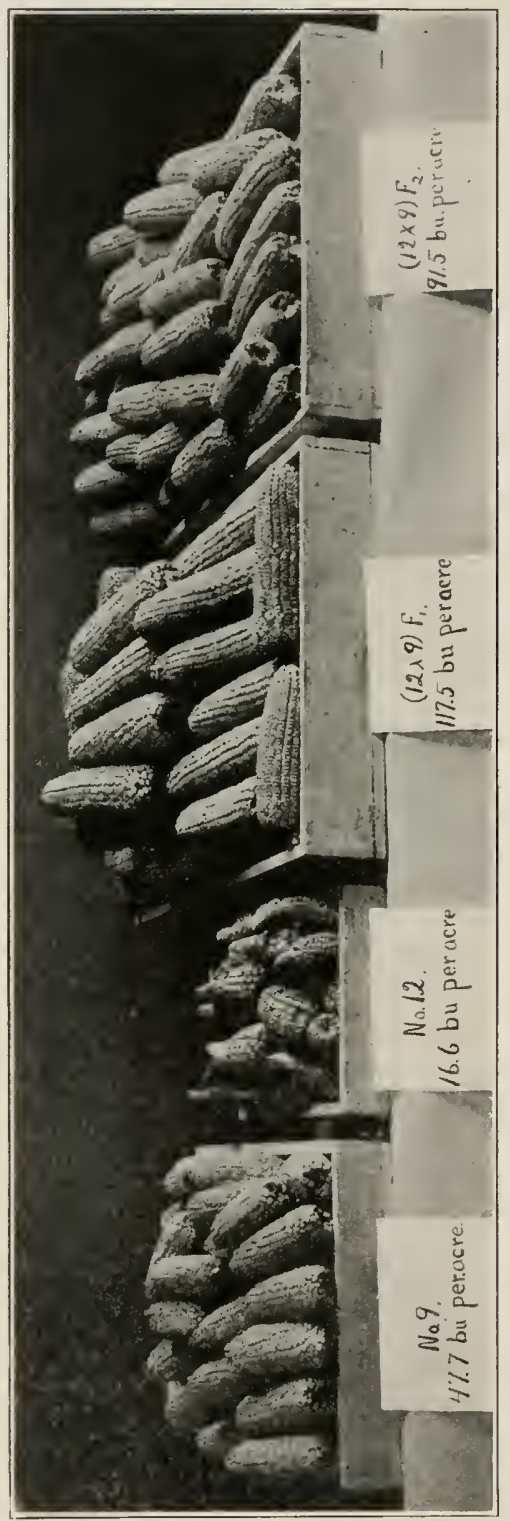
fertilized for a number of generations, the plants tend to become homozygous, to lose the vigor due to heterozygosity and to become smaller and less productive. This loss of vigor was for years interpreted as the direct effect of selffertilization. Now we know that it is simply the withdrawing of pure strains from hybrid combinations. In a few generations the strains become practically pure and the loss of vigor ceases. Some strains of maize still yield remarkably well after many generations of selffertilization. Other strains are so poor that they can scarcely be kept alive. In fact it is evident that they are kept alive merely by the increased vigor of growth due to continual natural hybridization with other strains. 
Since all commercial methods of selection in maize, as well as other naturally cross-bred species, have as their ultimate goal the isolation of good homozygous strains (for this is what the words "selection to type" mean), it is quite evident that the longer selection has been carried on the more of this stimulus due to heterozygosity or hybridity is lost. No method of breeding naturally cross-bred species therefore, where size and total yield are the main objects, is proper unless these facts are taken into consideration and the methods so modified as to utilize them. This is done by growing only the first hybrid generation of crosses between good strains. Nor is it alone in wind-pollinated field crops, such as maize, that these methods are useful. Horticultural crops such as tomatoes and eggplants can be grown from hand-hybridized seed with a profit greatly exceeding the extra cost of its production. It may be that even certain trees can be hybridized to advantage for undoubtedly Burbank's quick-growing walnuts are due to this phenomenon. Furthermore, it accounts for the fact that all asexually propagated crops worthy commercial supremacy, such as grapes and potatoes where yield is the object of prime importance, are always hybrids. Tleir mode of commercial propagation is such that the first hybrid generation can be indefinitely prolonged.

It is not easy to leave this subject without mentioning the important rôle which this growth stimulus clue to hybridity may have played in the evolution of the higher plants. In self-fertilized species, for example the violets, the fact that the hybrid between two nearly related strains was more vigorous than either parent type would have given it such an advantage in the ordinary struggles for existence against inhospitable environment, that the chances are greatly in favor of its surviving to produce recombi- 
nations of the parental characters in the next generation. And there is always the chance that new recombinations of parental characters may prove better fitted to survive than the old combinations. In cross-bred species the stimulus of hybridity holds a still greater advantage since even homozygous strains that are weak and could never exist alone, may, througn combination with other strains, be kept in existence as heterozygotes. For example, one finds in maize literally thousands of genotypic strains in a single commercial variety. Many could not exist alone, yet they continue to exist in commercial varieties through hybridity and their existence may be proven by their being partially withdrawn by inbreeding. Such strains may have great possibilities in certain combinations as is shown in Fig. 49. In inbred or self-fertilized species such as tobacco, however, strains weak in themselves perish and are lost to sight because there is no probability of their being hybridized and given a chance of showing their power in combination.

This one phenomenon, alone, may account for the commonness of cross-fertilized species and the rarity of selffertilized species, since it can be shown that there is no evil effect due to inbreeding per se.

Passing now to the work more generally included in plant breeding, we find that commercial methods fall naturally into two classes, hybridization and selection. They are not really thus separable since one must use selection after hybridization, but in the first category are classed all cases where man produces hybrids artificially. The main object of hybridization is the shuffling of unit-characters in the first hybrid generation and their recombination and fixation in succeeding generations. The object of selection is to withdraw from mechanical mixtures or from physio- 
logical mixtures due to hybridity, strains characterized by desirable new variations.

Practical procedure in hybridization naturally varies somewhat depending upon the exact object in view. I will endeavor to illustrate the following phenomena as those of most importance: (a) Recombinations of desirable characters and their fixations, including the production of blends; $(b)$ production of desirable combinations in the first

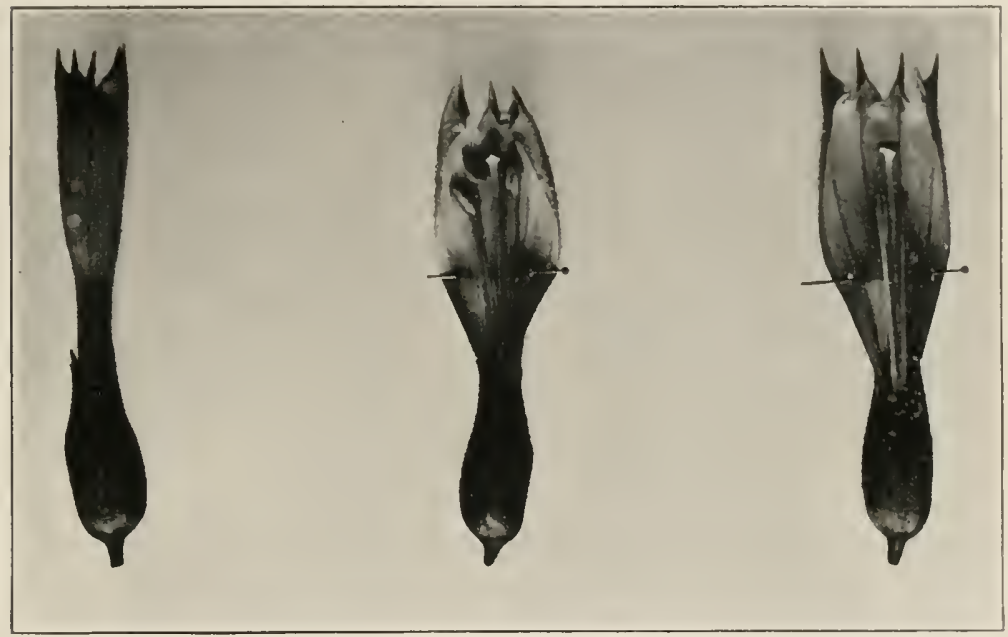

Fig, 50.-Buds of Nicotiana tabacum showing time and method of castration

hybrid generation and their continuation by asexual propagation; (c) production of fixed first generation hybrids.

If one is to begin at the real beginning in this discussion, he should spend a moment in describing the mechanical operations of crossing (Fig. 50). There are three important steps. First it must be determined by experiment what environmental conditions are best suited for normal seed production. Second, an intimate knowledge of the flowering habits and flower structure should be gained in order 
that the flowers may be castrated at the correct time without injury and properly protected from foreign pollination until the time for hybridizing. Third, care must be exercised in applying the pollen of the proposed male parent, for both premature and delayed pollination inhibits seed formation.

The precise conditions under which a cross should be made to be the most successful are not easily determined. The proper preparation of the breeding plot even before the plants are grown is necessary. One takes it for granted that on most soils some fertilizer will be used, for the plants must be normal to seed well. The three essential elements of soil fertility are nitrogen, potassium, and phosphorus, and to get the best results compounds of these elements must be present in proper proportions. First, available potassium must be present in quantities sufficient for the normal production of healthy roots, leaves, and stems, and a moderate excess will not be harmful. If nitrates are present in excess, however, vegetative growth will be overstimulated and seed production will be small. A lack of phosphorus will produce the same effect upon seed production, but for a different reason. Phosphorus is an essential constituent of the proteid compounds found in large quantities in the seed. If the plants are to be in the best condition for the production of good seed after crossing, therefore, the soil should contain just the right amount of nitrates for a normal vegetative growth, and a generous supply of potash and phosphates. The exact amounts must be determined by experiment for each soil and each species of plant.

External conditions that are also under partial control of the breeder are available moisture through irrigation and sunlight by proper spacing or artificial shading. 
Other necessary knowledge that can be obtained only from experience is, which are the best flowers on the plant to serve as parents of the cross and what is the proper time for their pollination. For example, in the grasses the first flowers that appear usually form larger, healthier seed than the later blossoms. In most of the Solanaccac, the petunias, browallias, etc., the exact opposite is true. The time when the individual flower is most receptive to pollen is even more narrowly limited. Both premature and delayed pollination is the cause of many failures and the optimum time should be accurately determined. Having exercised these precautions, it remains to study carefully the structure of the flower in order that it may be emasculated, i.e., the anthers removed before the pollen is shed, with sufficient adroitness that neither the anthers shall be opened nor the parts of the pistil injured. Only a few buds upon a single flower spike should be operated upon if they are to be given the best chance of development. If the buds are very small and some pollen unavoidably reaches them, it may be washed off with comparative safety with a dental syringe if done immediately. It is often recommended that the calyx and corolla be cut away when emasculating. This should be avoided if possible and the floral envelopes left as a protection to the pistil. After emasculation the buds should be protected from foreign pollen until time for pollination, and again after pollination at least until the fruits have begun to form (Fig. 5I). This protection may be an ordinary paper bag when the crossing is done in the field. It may be used with a plug of cotton around the mouth if special precautions are found necessary. In the greenhouse a square of thin celluloid rolled around the flower and caught with two rubber bands, each end being 
protected with absorbent cotton plugs, is a better device. It gives excellent protection and allows transpiration.

But I must pass from the technique of hybridizing to

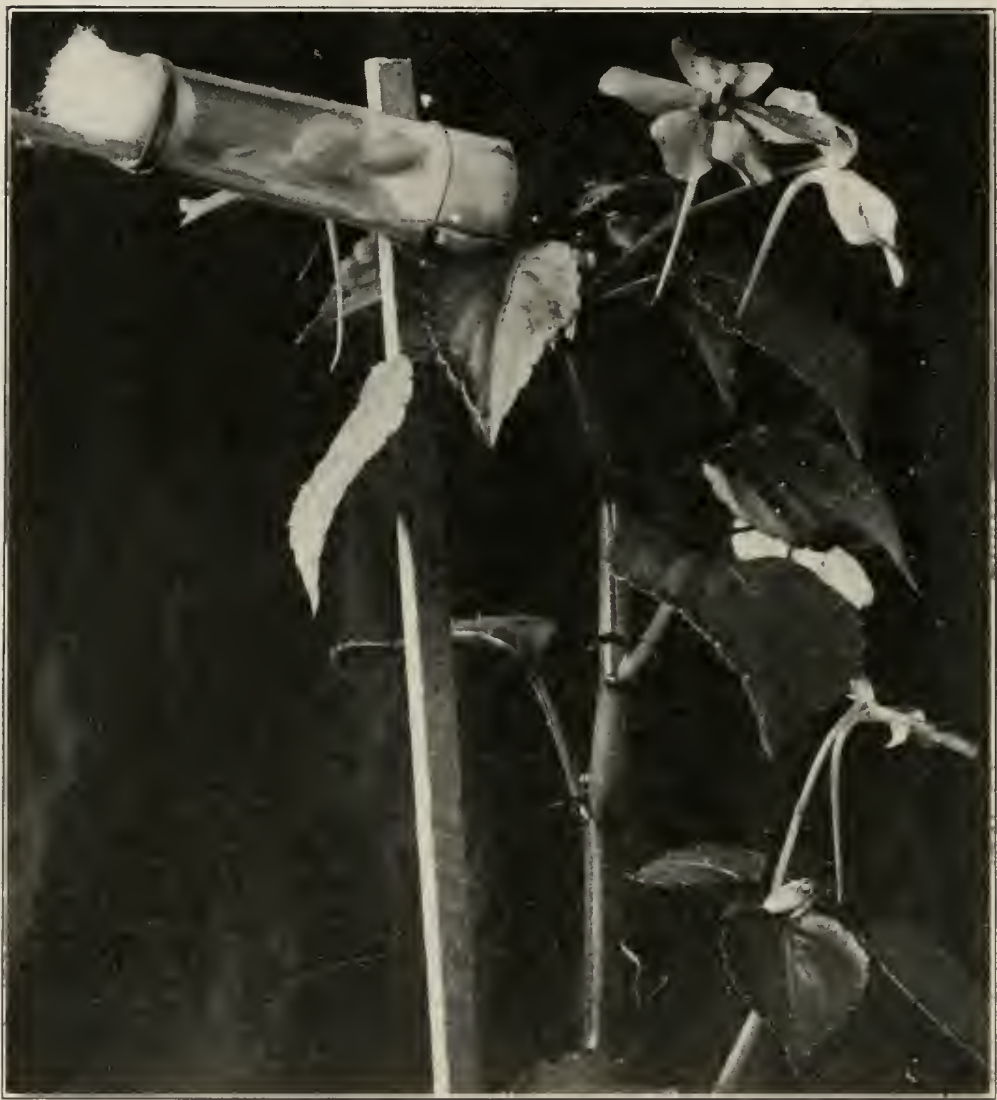

FIG. 51.-Impatiens sultani showing protection of castrated buds in greenhouse.

the results. They are much more interesting. As has already been stated, desirable horticultural novelties are obtained most frequently by crossing two plants which 
differ in several transmissible characters. The first hybrid generation may be as uniform as one of the pure varieties, but in the second hybrid generations all possible combinations of the characters of the two parents are obtained provided a sufficient number of individuals have been grown. Among these combinations many new and desirable types may be found. Some of them are pure types; some are heterozygous and will again segregate. Since homozygous and heterozygous types are found which are exactly alike in appearance, the only way to determine which plants are pure is to self-fertilize desirable individuals and raise a third generation. For example, $N$. alata, a species with large white flowers, when crossed with $\Lambda$. forgetiana, a species with small red flowers, gives hybrids that are very uniform in all their characters. The flowers are intermediate between those of the parents in size, and are red in color. In the second hybrid generation, there are 16 visibly different color types. Among these there are really $8 \mathrm{r}$ classes, including those both pure and hybrid. It is therefore necessary to grow seed from many self-fertilized plants for another generation to be certain of getting pure strains of each type. But having done this, the pure types continue to breed true in spite of the mixed ancestry.

This method is typical of the manner in which floral novelties are produced. So many varieties carry latent characters that one is always likely to obtain new things in crosses. Results of greater economic worth, however, are probably obtained by combinations made for a definite purpose. A beautiful example of such work is afforded by the experiments of Biffen. English wheats have long been known as highly productive varieties, but they are very 
susceptible to a fungus disease called rust, and do not make first-class bread on account of the low percentage of gluten. After many importations, wheats resistant to rust and high in gluten content were obtained, but these were not profitable bêcause of their low yields. Biffen then went

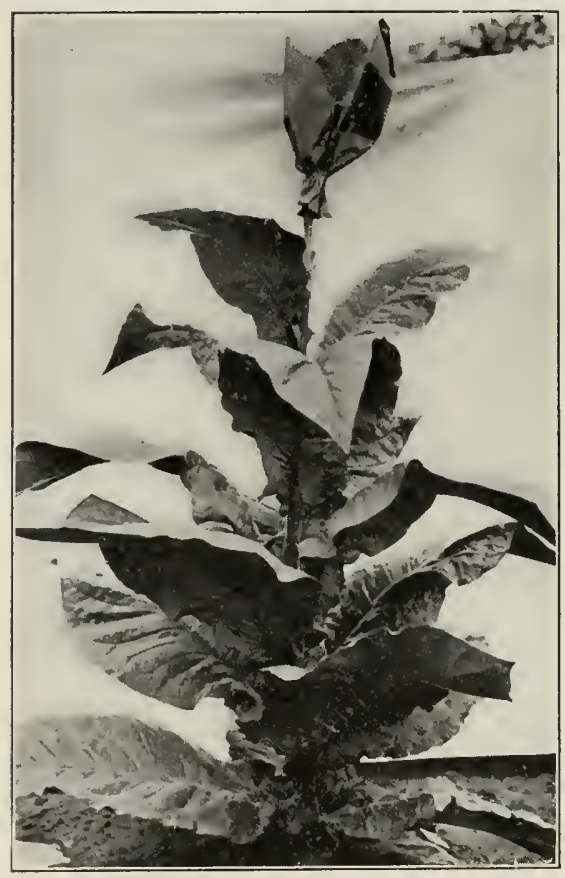

FIG. 52.- Vicotiana labacum variety "Havana." A stocky habit of growth with about 20 large leaves.

to work to analyze the transmissible characters of the wheats into Mendelian factors by a large series of crosses. This he was able to do. The rest was easy. He has now produced by hybridization wheats that comb'ine the desirable qualities and which lack those disadvantageous to the grower and the baker.

Sometimes a very simple recombination is of very great commercial value (Fig. 52). The socalled Havana type of wrapper tobacco grown in the Connecticut River valley has large leaves and a short stocky habit of growth. It produces I S-2 I leaves. There is another type from Sumatra which has tall habit of growth with about 26 comparatively small leaves. These two types were crossed by Shamel. From this cross a new type called the Halladay has been 
produced having the greater number of leaves of the Sumatra parent and the stocky habit of growth and large leares of the Havana parent (Fig. 53). The first interpretation of this result was that an entirely new variation had appeared for the Sumatra does not usually have as many as 26 leaves. The writer has been able to show, however, that the actual strain of Sumatra used as the parent had an average of 26 leaves, and data have now been collected which indicate that the new variety is a simple recombination of the characters possessed by the two parents giving a strain averaging 30-50 per cent greater yield than the old Havana variety (Fig. 54).

In a similar way Orton has combined the edible quality of the watermelon with the resistance to wilt of the citron or stock melon; Vebber has combined the

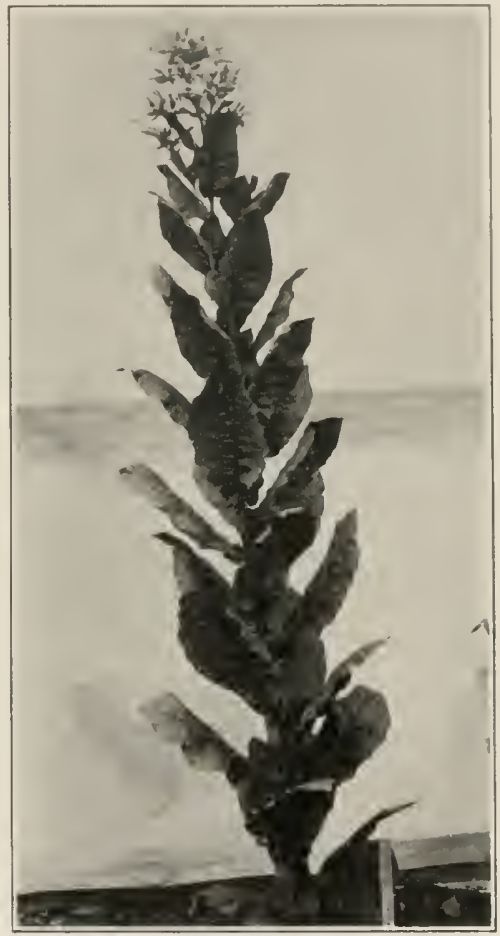

FIG. 53.-Nicotiana labacum variety. "Sumatra." A tall habit of growth with about 26 small leaves. fine, long, strong lint of the sea-island cotton with the large bolls and productiveness of the upland cotton; Price has made many new combinations in tomatoes; and ron Rümker has produced numerous valuable varieties of rye and barley. So the list might run on and on. Hundreds 
of plant breeders are using these methods to produce thousands of new types annually. Most of them are worthless, nearly all of them are no better than what are already in commercial use, but the comparatively few that are

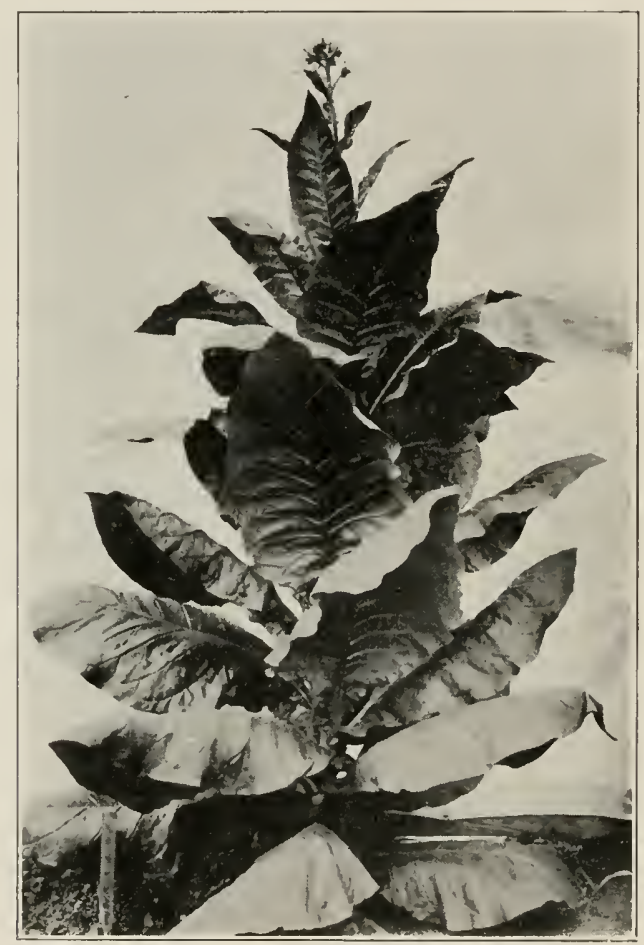

FIG. 54.-Hybrid combining the desirable qualities of the "Harana" and "Sumatra" varieties.

really superior repay the time and money spent a thousand fold. I might add that it is the community at large that is highly repaid, however, for the plant breeder, unlike the inventor, never gets rich through his productions. 
Recently, accurately controlled investigations have shown that a strict Mendelian notation will interpret results that hitherto had been given the name blended inheritance. For instance, one may cross an eggplant, Solanum melongena, bearing large fruits with one bearing small fruits. In the first hybrid generation, fruits intermediate in size are produced. Segregation in the second hybrid generation is such that plants bearing fruit like either parent can be obtained if a large number of individuals (several thousand) are grown. Yet among the $\mathrm{F}_{2}$ progeny, intermediates still occur in large numbers, and from them pure types can be secured.

Most of the characters hitherto described are qualitative in nature. They are either present or absent in the different varieties. Such characters are generally dominant, in which case the heterozygotes are like the homozygotes in appearance. Other characters give heterozygotes intermediate in appearance, owing to incomplete dominance, but these intermediates can never be fixed. Owing to their heterozygous constitution they always segregate the parental characters in the next generation. Size characters or quantitative characters, on the other hand, are often very complex. They are due to the interaction of many factors. For this reason blends may be obtained in the $F_{2}$ generation that are homozygous for such a combination of gametic factors that they always breed true to that condition (Fig. 55).

Fortunately it is not necessary always to have plants that breed true to seed. Many commercial plants are propagated asexually by bulbs, tubers, cuttings, etc. Here one has a method of growing portions of a single plant for an indefinite length of time. Fruit trees, bush fruits, 
strawberries, potatoes, pineapples, and many other kinds of economic plants belong to this category. This is a great advantage. One can so propagate homozygous strains if he wishes, but in addition he has a means of utilizing heterozygotes that would not breed true to seed and also of keeping

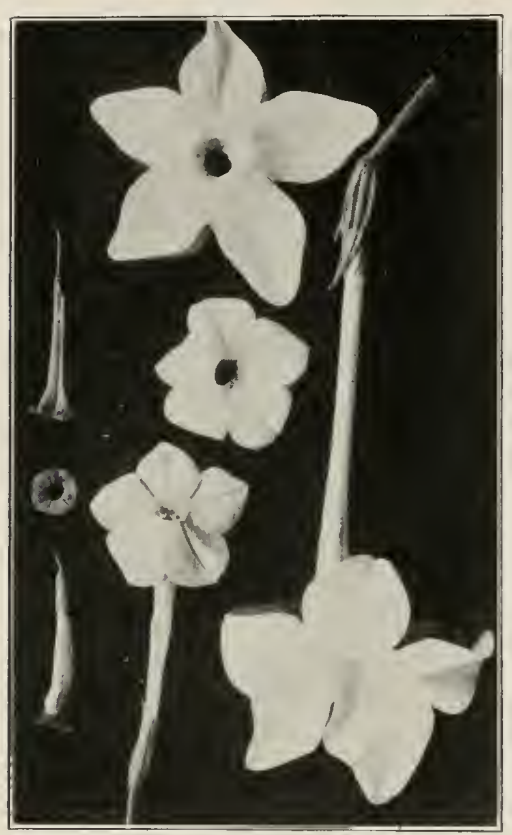

FIG. 55--Intermediate character of an $\mathrm{F}_{\mathrm{r}}$ hybrid. At left, Nicotiana paniculata; in center, hybrid; at right, Nicotiana alata. the greater vigor that accompanies heterozygosis.

No better example of such work can be given than that of Webber on citrus fruits. The great bugbear of the Florida orange grower is the frost that occasionally comes, leaving devastation in its wake. Webber, therefore, set himself the definite problem of producing a frost-resisting orange. $\mathrm{He}$ made several reciprocal crosses between the common orange and the hardy but worthless trifoliate orange (Citrus trifoliata). Among the seedlings obtained, several have proven valuable. They form a new class of citrus fruits and have been called Citranges. Three of these varieties have been named the Rusk, the Willits, and the Morton. The Rusk, which is a hybrid of orange crossed by trifoliata, is a small fruit with a bitter tang like the pomelo. It makes excellent marmalade and preserves. The Willits, coming from a 
cross of orange upon trifoliata, is a rough, but thin-skinned fruit, resembling an orange in appearance but a lemon in flavor. It is used as a condiment or for citrangeade. The Morton, coming from the same kind of cross as the Willits, is a large, juicy, almost seedless fruit, only slightly more bitter than the sweet orange.

Young trees of these three varieties have endured a temperature of eight degrees above zero, and it is thought that by the use of them and of similarly obtained varieties, citrus fruit culture can be extended fully 400 miles north of the present region.

In connection with this description of the production of new citrus fruits it may be well to mention that they are sometimes seedless. In fact, seedless fruits are often obtained by crossing. In true annuals reproducing by seed only, such productions would be of no value, for they would perish at the end of the first season. Seedless perennials, however, are among the most valuable horticultural varieties simply because they can be propagated asexually. In floral novelties, moreover, not only seedlessness but entire sterility is not a drawback to commercial worth, because sterile plants are often famous for their profuse flower clusters.

It was stated earlier that one phenomenon of hybridization was the production of fixed or constant first generation hybrids. This statement was made from hearsay evidence. There are several cases in which either new characters or blended characters that breed true appear to have been formed, but they have not been studied with sufficient care for their mode of inheritance to have been accurately and finally decided. In crosses between certain true species, hybrids have been produced that are 
seemingly very constant and uniform. Perhaps the most famous of these are the blackberry-raspberry hybrids first produced by the late E. S. Carman and later by Luther Burbank and others. Several hybrids having a commercial value have been made in this genus (Rubus), and the small number of second generation progeny that have been grown are said to have bred approximately true. Practically, it makes little difference about the exactness of this statement. One can simply say that for all ordinary intents and purposes, such hybrids breed true. To the scientist it makes a great deal of difference whether these hybrids are definite exceptions to the law of Mendel or not. The few data that we have are not sufficient to clear up this point, but several hypothetical explanations of the phenomena can be given that are in harmony with a belief in the universality of Mendelianism.

Nothing is really known about segregation in these hybrids because the variations that occur are difficult to describe and because the plants have never been grown in large quantities. It is likely that numerous separately heritable characters are concerned in such crosses between true species, and when $n$ pairs of character are concerned it takes four to the $n$th power seedlings to run an even chance that there will be one plant like each of the parents. When one considers that with ten pairs of characters, this means over I, $, 00,000$ individuals, he can see what enormous numbers are needed to give valid conclusions. Moreover, these hybrids are only partially fertile and some consideration must be given the possibility that selective fertilization among the gametes of the hybrid may occur. To take a hypothetical case, suppose two plants are crossed in which the flowers of one are twice the length of the flowers of the 
other and that the extra length of the longer flower is controlled by three or four separately heritable factors. If only a few of the egrg cells and pollen cells can fuse on account of the dissimilarity of their gametic constitution, one would expect only those seeds to be formed that would result from the fusion of the germ cells nearest alike. Intermediates would therefore be more likely to occur than extremes.

There is one other possible way of accounting for constant intermediate races. In crossing species of the genus Nicotiana, I have had plants develop from carefully guarded and supposedly hybrid seed that were exactly like the maternal plant. These seeds must have resulted from apogamy or polyembryony, that is, from the development of an immature egg cell without fertilization. The phenomenon was evidently induced by the extraordinary irritation of the foreign pollen. The question then arises: May not the difficulty of maturing sex cells in the $F_{1}$ generation of a wide cross sometimes cause apogamous seed development and therefore a continued propagation of a constant and uniform race?

These pieces of work illustrate the various distinct types in the improvement of plants by hybridization.

Intentionally, little has been said regarding the fixation of desired character combinations when the new varieties obtained are to be reproduced by seed. The reason for this omission is that the selective method used after hybridization is the same as that used upon crops whose small seeds and tendency to vary makes it difficult or unnecessary to produce artificial hybrids. The method is Vilmorin's and is based upon the fact that one cannot tell the most productive or otherwise desirable plant by inspection. The true basis 
of selection must be the average condition of the progeny of a plant determined by actual field tests. The entire object of selection is accomplished when a homozygous strain or strain genotypical for the desired qualities is isolated. The idea is simple; to put the idea into practice successfully is often a tedious and difficult task.

As in hybridization, the ease with which results can be obtained by selection depends largely upon flower structure. In selection, however, the relative facility with which artificial cross-pollination can be accomplished is of small importance. What one wishes to know is whether crosspollination or self-pollination takes place naturally.

Practically all plants are occasionally cross-fertilized naturally, and many of them have devices whereby they are nearly always crossed; but, as we have already seen, though cross-fertilization is an advantage to a plant, it is not at all essential. Wheat, for example, is almost always self-fertilized; yet it has kept its vigor for thousands of years. The importance of this fact to the selectionist is readily seen. If seed from several varieties of wheat is mixed and planted, each variety remains true to its type because of self-pollination, and each strain can be recovered in one generation. In like manner, if desirable variations occur in a wheat variety, it is a simple process to separate them from the parent strain for the two are mixed mechanically. It is only necessary to save seed from individual plants and grow them in separate rows or plots. One can see immediately whether the desirable variation is inherited or not, and if so the thing is done.

In a cross-pollinated plant the method is the same, but the work is not so easy. The pollen is carried through long distances by the wind or by insects, and even with carefully 
isolated plots the plants are often intercrossed. Each prize plant selected for future breeding will have had a few and possibly many of its orules fertilized by pollen from less desirable strains. When these seeds are grown they of course again fertilize the ovules of the desirable plants with a frequency proportionate to their number. In certain plants the process may be shortened by having recourse to artificial self-pollination. But unfortunately this cannot always be done. Suppose one were dealing with red clover where the flowers are small, almost sterile with their own pollen and produce only one seed. In such a crop a long and tedious method of continuous selection must be used for there is no other way. One must simply keep in mind the supporting principle of all selection work, that the seeds of single plants are grown in isolated plots and the character of the mother plant is judged by the characters of the progeny.

We have already seen from Mendelianism and the genotype conception of heredity why this method is the only proper one, but perhaps an illustration will show the matter more clearly. The older method of selection, called variously the German or "mass selection" method by plant breeders and the "performance record" method by stockbreeders, is based entirely upon the appearance or general character of the mother. For example, the German sugar beet raisers have for years analyzed large numbers of sugar beets and have grown their seed from the mother beets showing the highest percentage of sugar. No particular attention was paid to planting from "blood lines" of high sugar content; those beets were bred from which appeared to be the best by their performance record in the polariscope test. A great many of these selected mothers 
were simply high extremes belonging to "blood lines" that were low in their average sugar content. These individuals crossed with those from better "blood lines" and progress was made very slow indeed.

In this short discussion on selection the writer has endeavored to make clear two points that may be summarized as follows. Plants are exceedingly variable but the majority of these variations are simply accelerations or retardations of the development of the whole or of certain parts of the plant due to good or bad environment at critical stages of the plant's growth. These variations are not inherited because the reproductive or germ cells are not affected. Other variations, however, are being constantly produced by nature - though much more rarely-which do effect the reproductive cells and are transmitted to the plant's progeny. These variations are the basis of selection. They are constant from the beginning-although their possible presence in a heterozygous condition may make it seem otherwise -and remain so unless changed by a second variation affecting the same constituent in the reproductive cells that is due to develop the character in question. The second point to be emphasized is that the whole aim and action of selection is to detect the desired heritable variants among the useful commercial plants and through them to isolate a race with the desired characters. When such a homozygous race is produced, selection can then do nothing until nature steps in and produces another desirable variation. The progeny test is the way to accomplish this end. It does this by showing us to which strains each mother plant belongs. It is a sure test whether the heterozygous condition is simple or complex. If it is a question of which seeds of a maize ear are homozygous and 
which are heterozygous for starchiness the matter is cleared up at once. If it is a question of which of a lot of beans is homozygous for the gene-complex of large size, the complexity of factors concerned may require a greater number of progeny, but the test is valid in the end.

There remains for mention a phenomenon of some interest eren though it has produced few varieties of plants of commercial importance. This is the sudden appearance of a branch with characteristics different from the mother plant upon which it is borne that may be cut off and propagated asexually. It is the so-called bud sport. It is of practically no importance outside of the production of floral novelties. Perhaps this is accounted for by the fact that bud variations nearly always affect the same characters that have previously been changed in the same way through seed variations. Furthermore, the change is practically always the loss of a character which leaves little opportunity for the production of the real novelties through progressive variations (Fig. 56).

The production of the smooth-skinned peach, the nectarine, as a sport from the ordinary peach tree, is the classical example of this type of variation. This is undoubtedly simply the loss of the Mendelian factor for presence of the down upon the fruit, and might be expected to come about through some abnormal cell division in much the same manner that variations occur in the reproductive cells. They are usually not inherited through the seeds. This is what would be expected. It is said, however, that sometimes such variations come perfectly true to seed. If this is so, one must suppose that the varying plant cell was one which could give rise to the reproductive cells. Since nothing definite is known of this matter, however, speculation does 
not seem wise. It is simply mentioned as one other way in which new plant varieties originate.

In conclusion I wish to anticipate a possible question.

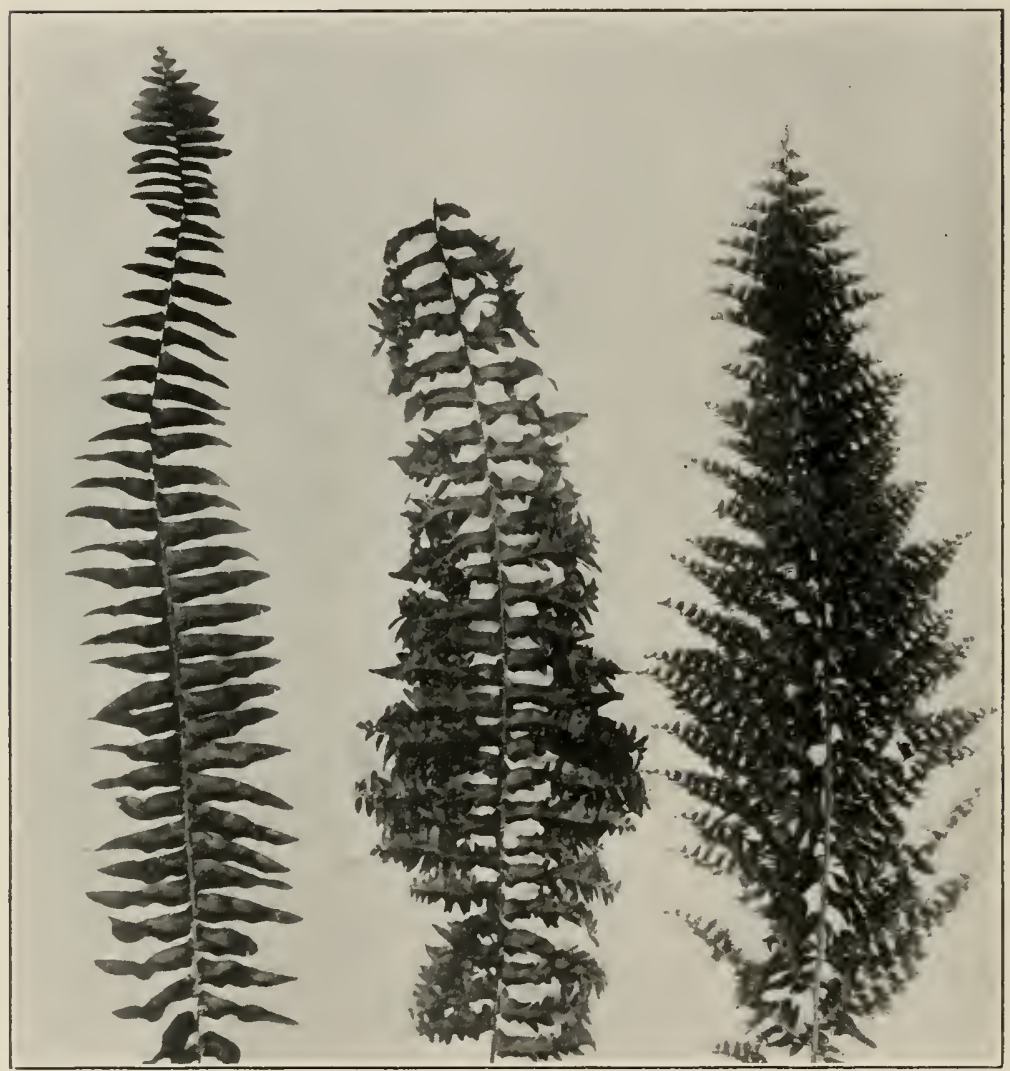

FIG. 56.-Frond of Boston fern and types that have arisen through bud variation.

What help have the new biological principles been to the commercial plant breeder? A prominent horticulturist has said that the new discoveries have made necessary no changes in method. With the exception that they have 
shown the scientific basis for Vilmorin's method of selection, which before was not in general use, the statement is largely true. Yet they have been of inestimable practical value. They are time savers. In hybridization, one no longer grows large quantities of first generation hybrids and small quantities of second generation hybrids, for he knows that it is in the second generation that the desirable recombinations of characters will occur. In many cases he can even predict with some accuracy the exact number of second generation individuals which it will be necessary for him to grow to obtain the desired result. And even when this cannot be done he knows that the blended characters of the first hybrid generation do not mean that he has failed to attain his object. It is simply a matter of growing large numbers in the second hybrid generation that insures success. Furthermore, the plant breeder has a means at hand to show what characters are heterozygous and therefore unfixable. He therefore no longer wastes time in striving for a pure strain of a heterozygous type such as the Blue Andalusian fowl. Nor does he still regard the appearance of "rogue" plants in his nursery beds as a necessary affliction of Providence. He has learned that they are simply recessive segregates and can be prevented by properly protected hand-pollinations.

In the field of selection the new ideas are still more economical of time. To the belief that faith and continuous selection toward an ideal would produce any desired result has succeeded the idea that nature alone produces variations and that man's duty is to be alert to grasp their possibilities and to make the most of them. No longer is it believed that many generations of work are necessary to purify a commercial variety of plants from undesirable 
characters. No longer is there belief that the results of selection are continuous, that it gradually perfects a character. We work for strains homozygous for characters that we know are there, and, by our direct methods we get them without loss of time. 


\section{WILLIAM LAWRENCE TOWER}

Associate Professor of Zoölogy, the University of Chicago 



\section{CHAPTER VII}

RECENT ADVANCES AND THE PRESENT STATE OF KNOWLEDGE CONCERNING THE MODIFICATION OF THE GERMINAL CONSTITUTION OF ORGANISMS BY EXPERIMENTAL PROCESSES

\section{INTRODUCTION}

Through inheritance there comes the rhythmic repetition in each specific organic form of a precise and definitely repeated series of erents ending in the production of an individual which in time sets free from itself a highly organized and specific mass - a gamete, which, when properly combined with some other gamete and nurtured, repeats again the series of events which took place in the parent bodies which preceded them. Throughout all of this complex, rhythmic process a material basis, the germ plasm, keeps intact from parent to progeny genetic lines of descent. This continuity of material basis-first clearly recognized by Gustav Jaeger, and later woven by Weismann into his germ-plasm theories-while known in its gross appearance and many of its behaviors, is nevertheless quite unknown as regards its real constitution and the means through which it does reproduce so accurately in each generation the sequence of events characteristic of its specific organic form.

The purely a-priori hypotheses of the "constitution" of this substance in reality help us little or not at all as a basis for experimental investigation. The id-determinantbiophore fabric of Weismann, Naegeli's micellae chains, 
DeVries' pangene complex are no better and perhaps no worse than the hypothesis that racial memory is the basis of inheritance, or that the complex or harmonious equipotential systems of Driesch "explain" the phenomena.

As far as the facts of development and heredity are concerned, they might go on indefinitely repeating any given series of events, but there would be only one type of organism, alike at any and all points in the genetic chain. Adequate evidence that there have been changes in this series of events in the past and that changes are now going on is found in the array of specific organic forms that exist and have existed through geological history. How have these changes been produced?

The nineteenth-century biology formulated its hypotheses around two widely different concepts; an extreme transmission hypothesis in which modifications arising in peripheral parts - soma, i.e., personal peculiarities developed in life-are transmitted to the progeny, through being in some manner incorporated into the germinal constitution of the race; and the hypothesis that changes in the race arise primarily in the germinal substance itself and appear later in the soma.

The idea of the peripheral origin of variations through the stress of the conditions of life dates back to Buffon, Erasmus Darwin, and Lamarck, whose ignorance of things now common knowledge had led them to express the opinion, backed by much circumstantial evidence, that the conditions of life, especially when changed, produced variations which were heritable in the race. Darwin advanced the hypothesis of an atomistic mechanism whereby this transmission could conceivably be produced and upon this provisional hypothesis of Pangenesis have been based all 
subsequent hypotheses of the peripheral origin of modifications and of their inheritance.

The hypothesis of the peripheral origin and transmission of variations is shown in diagram in $\mathrm{Fig} .57 A$, where the cause of variation ( $x$ ) impinges upon the organism and

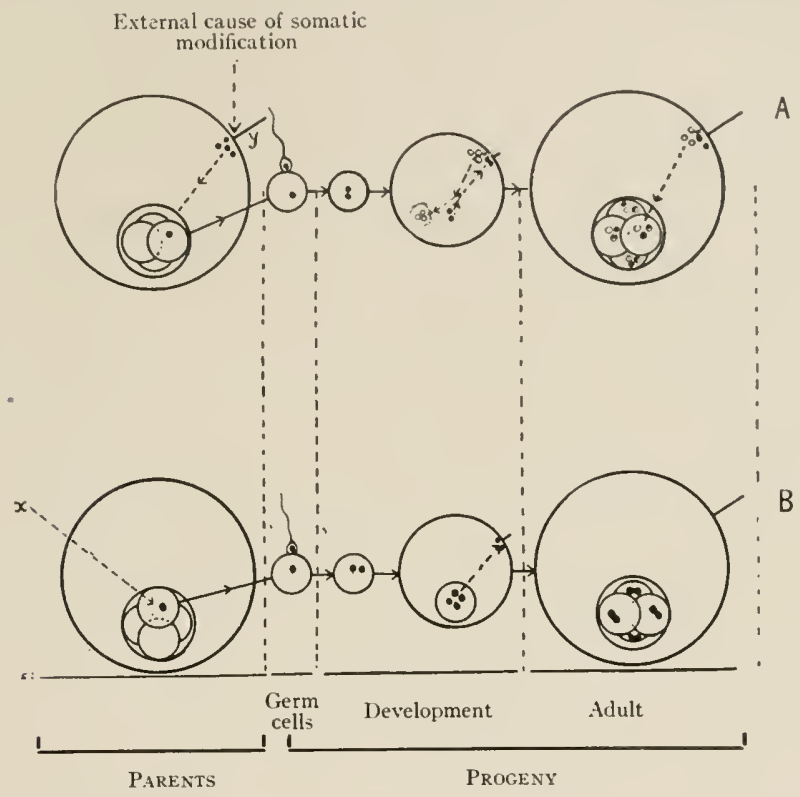

Fig. 57.-(A), Diagram to represent Darwin's Provisional Hypothesis of Pangenesis and current neo-Lamarckian conceptions and (B), the continuity of the Germ Plasm Hypothesis of Weismann.

induces a change, variation $(y)$, and from the cells composing this modified part, as from all other cells of the body, gemmules, minute masses of matter, electrolites, or something, are thrown off and these units conserve the power of reproducing the replica of the part from which they come, either normal or modified. These gemmules are supposed to be gathered in the gametes, and in reproduction are supposed 
to be redistributed peripherally and to reproduce the duplicate of the particular character from which they arose. If now ( $x$ ) impinging upon the organism, gives $(y)$ a new variation, then new sorts of gemmules are supposed to be formed, and these on being gathered up and carried along in reproduction by the gametes will cause to reappear in the progeny the modified character. Repeated impact of $(x)$ may, in the opinion of the adherents of the view, successively increase $(y)$. All theories of the peripheral origin and inheritance of variations are patterned after Darwin's hypothesis, and although they have different expressions or terms for the carriers of the variation: nerve force, force, ions, electrolites, energy, etc., they are in essence the same conception and are all operated by the same mechanism.

Radically opposed to this theory of the peripheral origin of variation is that of the central or germinal origin of variation, in which the cause $(x)$. acts upon the germ and produces the change in the germinal constitution which, when the germ undergoes development, produces the divergent character $(y)$, the variation (Fig. ${ }_{57} B$ ).

Much logic has been expended upon this problem of transmission. Weismann has made a masterly analysis of the situation and can discover no reason for any conclusion other than in favor of the germinal or central origin of all variations that are efficient in evolution. Spencer, Cope, Eimer, Semon, Rignano, and others have tried to equal Weismann's logical analysis of the problem, but without any conspicuous success. The problem is one for experiment and not for solution by logic.

Very tiresome are the multitude of arguments, and the arrays of "plausible instances," and of the "facts" which "can only be explained" thus and so. Many times has the 
whole field been gone over and summarized, and yet one sees no progress, nor can progress be expected from this method of attack. Only precise experimental procedure can in any way aid in the solution of the problem, and all too often the experiments to test these theories, especially the earlier ones, admit of diverse interpretations, so that they are in the main inconclusive.

In this chapter are presented some of the data accumulated in recent efforts to gain precise experimental knowledge of how germinal changes are brought about. I shall, therefore, present in two divisions the data and conclusions bearing upon the two supposed methods of change, with such brief discussion and correlation of the already overdiscussed and over-correlated literature as may be necessary.

\section{THE TRANSMISSION OF SOMATIC VARIATIONS}

It has been proved that variations do arise primarily in the germinal substance, and appear secondarily in the soma; but can it be proved that modifications arising in the soma are transmitted to and incorporated into the germinal constitution and appear in subsequent generations? It is apparent that properly planned and conducted experiments are alone of service in the attempt to solve this question.

It in no wise strengthens the position of the supporters of the theory of the peripheral origin of variations to present an extensive array of examples not explicable excepting through the use of this idea, and even though it "does explain" and "may explain" a huge array, or even all of the problems of evolution, it does not thereby become a proven truth. Special creation equally well explains all the phenomena, if certain assumptions be accepted as true. However, not until this or any other form of transmis- 
sion can be obtained in properly guarded experiments and reproduced at will, can the process be admitted as a true evolutionary process.

The problem, therefore, is to produce "somatic variations" in a soma at such a time, or in such a fashion, that the germ cells will not be affected by the action of the incident forces used, and then by breeding discover if the change appears in the progeny arising from the unstimulated germs. Evidence of somatic influence upon germinal material may also be obtained by transplanting germ glands, especially ovaries, into different somas, as has been done by several experimenters. ${ }^{\mathbf{x}}$

The recent experiments of Guthrie, ${ }^{2}$ Castle, ${ }^{3}$ and Davenport ${ }^{4}$ are well adapted to showing any possible action of the soma upon the germ. In Guthrie's experiments proper care was apparently not taken to determine the character of the stocks used and to preclude the possibility of regenerated ovaries. Therefore his results are not conclusive. Guthrie describes his experiments in the ingrafting of ovaries between young females of single-combed black and singlecombed white leghorns, as follows:

During the summer of 1904 I exchanged the ovaries between two black and two white leghorn pullets, weighing about $650 \mathrm{gms}$. each. One black and one white pullet were saved for controls. All did well for some time after the operations, but during the winter, before the laying season began, their condition became extremely poor, owing largely to being kept in inappropriate quarters.

${ }^{x}$ Castle has recently given a comprehensive résumé of the ingrafting of germ glands to which reference should be made for more detailed consideration. IV. E. Castle and J. C. Phillips, On Germinal Transplantation in Vertebrates, Carnegie Institution of Washington, No. 144, I9I I.

${ }^{2}$ C. G. Guthrie, "Further Results of Transplantation of Ovaries in Chickens," Jour. Exp. Zöol., V (1908).

3 Ibid., I 9 I I.

${ }^{4}$ C. B. Davenport, Proc. Soc. Exp. Biol. and Med., VII (rgro), 168. 
On August 25, I906, another series of pullets of the same strains were similarly operated upon, controls being saved as before. They weighed about $75^{\circ}$ gms. each, the white ones being slightly the heavier. All did well after the operation. No marked differences in egg production were found between the control and operated hens, nor in the fertility of the eggs. The operated hens at the beginning of the laying season were somewhat lighter than the controls. In other respects no differences were observed in either the hens, eggs, or chicks.

The eggs became fertile in two to four days after mating and on cessation of mating the eggs became infertile in eleven to nineteen days, the majority becoming so orr the fifteenth day. Control hens $B_{I}$ and $W_{I}$ ) mated to the rooster of the same breed gave uniformly black fetuses and chicks in the case of the black hen, and white fetuses and chicks in the case of the white hen.

The normal black chicks had grayish-yellow breasts and throats and frequently the under surface of the tops of the wings was light colored as well, but the plumage of the entire dorsal surface was always solid black. The light-colored areas on the ventral surface were uniformly black after the first moult. Occasionally a normal black may retain one or several white feathers in the tip of the wing permanently, but this is of rare occurrence and such white feathers have not been observed in any other situation.

The normal white chicks were pure white to light buff when hatched, but after the first moult they were always pure white. The black hen $\left(B_{2}\right)$, carrying an ovary from a white hen $\left(W_{2}\right)$ mated to the white rooster, gave about equal numbers of white and spotted fetuses and chicks. (In all cases of very small white fetuses, spots may hare been overlooked.)

The white hen $\left(W_{2}\right)$, carrying an ovary from a black hen $\left(B_{2}\right)$ mated to the white rooster, gave white, black, and spotted fetuses and chicks. The spotted ones outnumbered the others combined.

The black hen $\left(B_{2}\right)$, carrying an ovary from a white hen $\left(W_{2}\right)$ mated to the black rooster, gave ordinary black, and black fetuses and chicks with white legs, in about equal numbers. In regard to the chicks from this hen described as ordinary black, some doubt exists as to whether the ventral light-colored area described for normal black chicks was not lighter and greater in extent in all cases than in the normal chicks. 
The white hen $\left(W_{2}\right)$, carrying an ovary from a black hen $\left(B_{2}\right)$ mated to the black rooster, gave uniformly spotted chicks, i.e., white chicks, with black spots on the dorsal surface of the head, neck, wings, back, or on the tail.

Owing to the uniform results from the controls, it may be assumed that the strains of chickens used breed true to color. Therefore any variations in the offspring of the operated hens were due to other influences.

The fact that in all cases of the operated hens white or black or spotted fetuses or chicks were produced (i.e., the offspring showed variations from the normal in color markings) shows:

I. That the eggs from each of the operated hens were from the transplanted ovary. Take hens $B_{2}$ and $W_{2}$. These hens were bred to the roosters of their color. Had some portion of their own ovary not been removed at the time of the operation (a remote possibility) and was functioning, then we would have expected solid offspring like the controls. But such was not the case. In the offispring from $\mathrm{B}_{3}$, in which the male and foster mother were black, black predominated but white occurred. This must have come through the white ovary. In the offspring of $W_{2}$, in which the male and foster mother were white, white was the predominating color but black occurred. The black therefore must have come from the black ovary.

If we accept the statement that in ordinary crossing of black and white breeds the white is dominant, then we assume that the same is not true for this kind of (female) crossing, or that the original color influence was more strongly preserved in the black than in the white ovary. From the constancy in the results in the above two hens, we may conclude that the ovaries transplanted into the other two hens, $\mathrm{B}_{2}$ and $\mathrm{W}_{3}$, were the ones functioning during the laying season also.

2. The foster mother exerted an influence on the color of the offspring. Take hens $\mathrm{B}_{2}$ and $\mathrm{W}_{3}$. These hens were bred to the rooster of the opposite color, i.e., the color of the transplanted ovary. Yet in the former the majority, and in the latter all of the offspring were spotted, i.e., white with black spots on the dorsal surfaces. In $\mathrm{B}_{2}$ the male and ovary were white and the foster mother black; in $W_{3}$ the male and ovary were black and the foster mother white. In both cases white predominated in the offspring. It would seem, therefore, 
if we leave the question of dominance out of account, that the fuster influence of the white hen was stronger than that of the black hen. If, on the other hand, we consider the foster influence equal in both cases, then we can explain the results as due to the dominance of the white in the male or ovary.

Guthrie's contention is that the ovaries were wholly removed, and did not regenerate; that the ingrafted ovaries developed, functioned, and were influenced by the foster soma to produce changes in gametic constitution. The doubtful points are concerning the nature of the stock and its gametic make-up, which was not tested in adequate manner, and the gametic constitution of all fowls is known to be very complex (cf. Bateson, Saunders, Davenport, and others); and the possibility of regenerated ovaries. Davenport has repeated the experiments on other but well-known stocks, and summarizes his findings as follows:

To test these experiments [Guthrie's] I transplanted ovaries from a cinnamon-colored, heavy-boot, pea-combed, low-nostriled hen which breeds true to a white, non-boot, V-combed, five-toed, high-nostriled hen, and mated her with a cock whose characters resembled those of the hen from which the eggs had been borrowed. Had the engrafted ovary been functional, the chicks must have all been like the cock. Actually, they were exactly what expectation calls for when such a cock is mated to such a hen like the so-called foster mother. The engrafted eggs are not functional; the ovary had degenerated.

Six experiments of this sort were made altogether and in no case was there eridence of a functional graft; far less of an influence on the eggs of the foster mother's soma.

These experiments, made on better-known stock, with many sources of error constantly in mind, give exactly contradictory results. Equally convincing and identical in result are the investigations of Castle in guinea-pigs, where no effect of the foster soma upon the ingrafted orary was 
found. Castle has made extensive transplantations of ovaries, especially in guinea-pigs, and finds that:

Out of seventy-four cases six died as the immediate result of the operation; four of these were cases in which a ventral incision was tried.

Summarizing the results of his operations he finds that in the results of the entire series only one grafted animal had young from her grafted tissue; grafted ovaries functioned in six other cases, but did not produce young. Ten animals regenerated their own ovaries, and three of these had young. Forty-two showed post-mortem complete atrophy of the genital tract and absence of ovarian tissue. The remainder comprises fifteen cases in which results were not fully determined.

On January 6, I909, the left ovary was removed from an albino guinea-pig (Fig. $58 B$ ), then about 5 months old, and the ovary of a pure black guinea-pig about a month old (Fig. 58A) was fastened near the tip of the uterine horn, distant a centimeter or more from the site of the ovary removed. One week later, January I3, a second operation was performed, in which the right ovary of the albino was removed, and as a graft was introduced the ovary of a second young black guinea-pig of like age with the first but of different ancestry. After the albino had fully recovered from the second operation she was placed with an albino male (Fig. $58 \mathrm{C}$ ), with which she remained until her death about a year later.

On the $23 \mathrm{~d}$ of July, Ig 8 days after the first operation, she gave birth to two female young. One was black, but bore a few red hairs. A photograph of this animal at the age of two or three months is shown in Fig. $58 D$. The other young one was likewise black, but had some red upon it, and its right forefoot was white (Fig. $58 E$ ).

On October 15 the grafted albino bore a third young one, a male, which, like those previously born, had a few red hairs interspersed with black. A photograph of this animal is shown in Fig. $58 F$.

On January ir, I9Io, the grafted albino was observed to be pregnant for the third time, and this time she was very large. Unfortunately, on February 2, she died of pneumonia with three full-grown male young in utero. The skins of these animals were saved and a photograph of them is shown in Fig. $5^{8 G, H}, H$, and $K$. Like the other three 


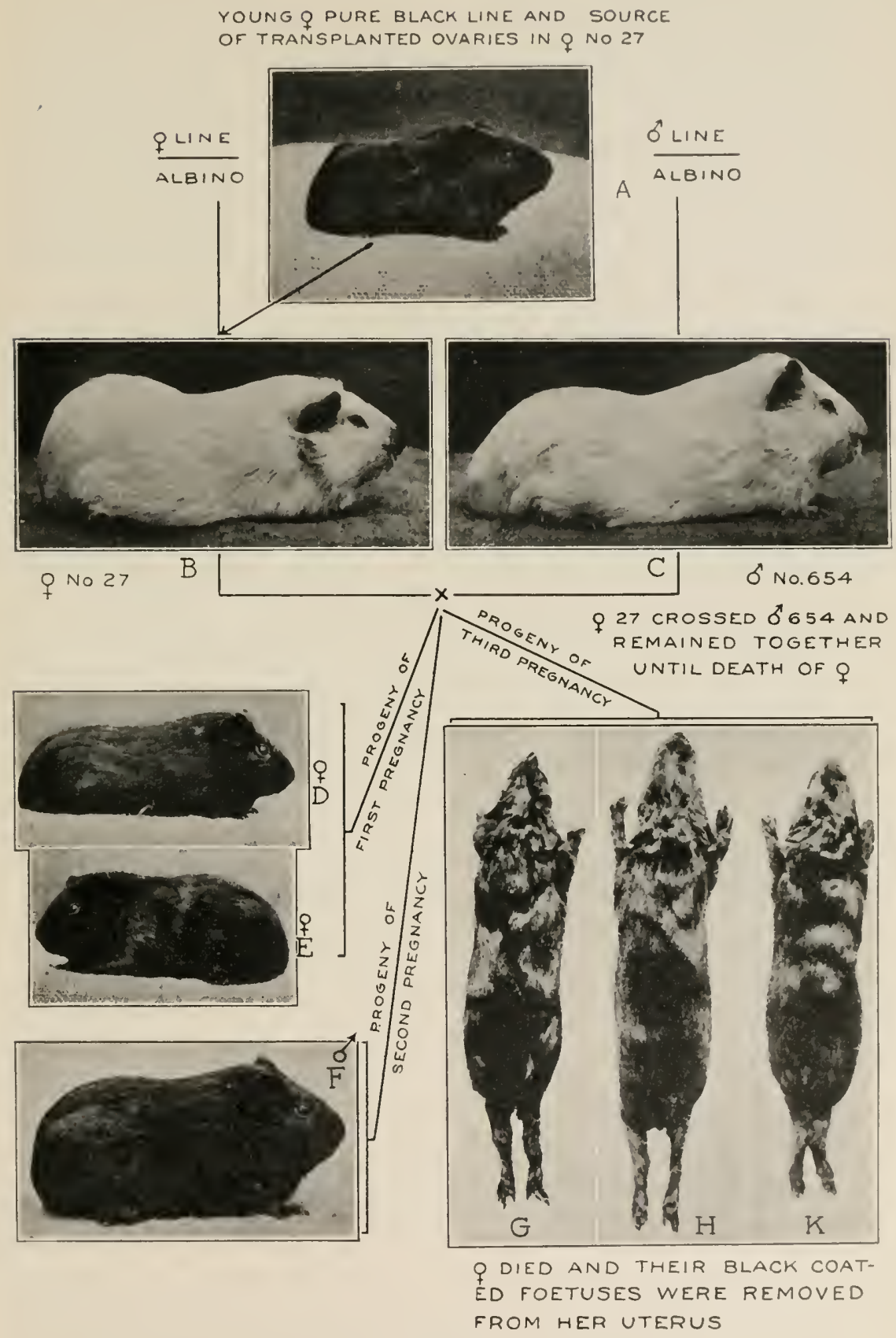

FIG. 58.-To show the lack of effect of foster soma upon introduced ora. (Modified from Castle.) See text for further cliscussion of this figure. 
young they were black, but with a few red hairs among the black ones. They bore no white hairs.

An autopsy made an hour after the death of the mother showed on the left side a distinct ovarian mass about a centimeter from the coiled part of the oviduct; that is, approximately the position where the graft from the pure black guinea-pig was fastened at the first operation. On the right side the mesentery of the oviduct was adherent to the body wall where an incision had been made at the second operation, and a small amount of tissue, regarded as possibly ovarian, was there observed. The tissue from the left side was found to contain numerous large egg follicles, some already well advanced, containing a lymph space; in addition a number of corpora lutea were observed. On the right was found a small amount of undoubted ovarian tissue, with one well-advanced egg follicle, but the whole apparently was strongly encapsulated, so that no eggs could be discharged even if they came to maturity.

It is interesting to note that both grafts persisted, though taken from different animals and transferred at different times. This result suggests a possible susceptibility on the part of the animal grafted.

Female I,970, a daughter of the grafted albino, was mated with the albino male, her father, and bore three young, two of which were albinos and one black with some red hairs. If female 1,970 had been the daughter of a pure black mother, instead of a grafted albino, we should have expected her to produce an equality of black and albino young. The observed result was the nearest possible numerical agreement with this expectation.

A control mating of the albino male was made with a female of pure black stock. As a result there were produced two litters of young, including five individuals, all black, but with red hairs interspersed. This result shows that the red hairs found on the six young of the grafted albino, were due, not to foster-mother influence of the grafted albino, but to influence of the male parent. The young of the grafted mother were exactly in color such as the black guinea-pig which furnished the graft herself might have been expected to bear had she been mated with male 654 instead of being sacrificed to furnish the graft. The white foot borne by one of the young forms no exception to this statement. Spotting characterized the race of guinea-pigs from 
which the father came. He himself was born in a litter which contained spotted young, whereas neither the pure-bred black race that furnished the graft, nor the albino race that received it was characterized by spotting.

Inasmuch as the offspring of albino parents are invariably albinos, it is certain that the six pigmented offspring of the grafted female were all derived from ova furnished by the introduced ovarian tissue taken from a black guinea-pig. This tissue was introduced while the contained ova were still inmature, and it persisted in its new environment for nearly a year before the eggs were liberated which produced the last litter of three young. These young, like the earlier litters, gave no indication of foster-mother influence in their coloration.

The conclusion is forced upon us that the egg cell during its growth does not change in germinal constitution. Its growth is like the growth of a parasite or of a wholly independent organism; what it takes up serves as food; this is not incorporated merely in the growing organism; it is made over into the same kind of living substance as composes the assimilating organism.

In all of these transplanted germ glands, it is true, as Castle recognizes, that his evidence and that of others does not disprove the possibility of foster-soma influence, but it is certain that the evidence is at present entirely against such influence. There are, however, two possibilities present in these experiments which should be kept clearly in view:

I. The transmission of some character from the foster soma to the germ and its incorporation therein.

2. The power of the foster soma to produce new surroundings as a result of the transplantation, thereby arousing new physical or chemical activities incident upon the transplanted germ cells.

If the first effect occurs as a result of the transplantation, it is to be expected and must be proven that some essentially entire characters are introduced from the foster soma into the alien germ cell and this seems in all critical experiments 
(Castle's, Davenport's) not to have taken place. As for Guthrie's experiments with poultry, it seems as if Davenport's adequately controlled and carefully repeated experiments gave results which show very clearly that Guthrie's cases are due to regenerated germ glands and impure stocks, and not to foster-soma influence. The second possibility, however, is a far different one and would show only variations of gametic constitution of the alien germs and not introduced characters. In other words, there is this fundamental difference between the two possibilities: the foster soma may act merely as a new environmental complex providing new physical and chemical states which may modify the physiological activities of the ingrafted germ cells; and this is very different from the conception of the foster soma as formulating "a something" bearer of its characters or character, which "something" it transmits to the germ, which "something" then reproduces in development the replica of the somatic part or character from which it came.

Neo-Lamarckians reply to the results obtained from these grafting experiments by the statement that the transplanting of ovaries is highly abnormal and would not occur in nature, and would not be repeated in sequences long enough to get the kinetic effect necessary to induce germinal change, and therefore the experiments in no wise satisfy the requirements of their hypothesis.

The idea of repeated impacts producing an accumulated kinetic effect only after long periods of activity finds most complete expression in the curious theories of Rignano, ${ }^{x}$ which, however, are only another logical subterfuge to

${ }^{1}$ E. Rignano, Sur la transmissibilité des caractères aquis. Paris, 1906. Also transl., Open Court Pub. Co., I9II. 
maintain the cherished dogma of the biogenetic repetition of ontogenetic stages and inheritance through a transmission of some kind. Adequate answer to these hypotheses would seem to exist in the non-inheritance of modifications of nose, ear, lips, etc., of many savage tribes, often repeated with intense kinetic effects of pain and stimulation, or of the feet of Chinese women, bandaged and modified through long series of generations, but these to the earnest neo-Lamarckian are mutilations and of course are not to be expected to be inherited. Curiously enough the "idea" only "works" in those instances where there are no facts or evidences available for analytical investigation.

Distinctly different from the results of grafting experiments or the arguments from plausible interpretations of past series of phylogenetic states, are the interpretations placed upon many experimental series not properly guarded. Thus Semon's interpretation of the results of many experimental series is justified from his point of view because so many investigations have not been sufficiently critical in orientation, nor in analytical procedure. Thus, for example, the experiments of Standfuss, Fischer, Pictet, Woltereck, Kammerer, Pshibram, Zederbauer, and others admit of interpretations from either point of view. What is unquestionably shown is change in gametic constitution, permanent and heritable, but not capable of answering the fundamental questions involved.

That organisms may be modified by incident conditions there is no reasonable doubt, but the question is, how? If the discovery of the methods of change is desired, then experiments made upon known materials under carefully guarded conditions are necessary, and are our only means of obtaining real knowledge of the underlying processes. 
It is obvious that progress in the solution of the problem can be made only through experiments based upon known materials which must meet certain rigorous requirements. The experiments of many observers with plants and animals show clearly that changes are produced which are inheritable in following generations, but do not produce accurate data upon critical theoretical points. Thus, for example, Sumner's recent work on mice is entirely of this order and gives only unreliable results. Nor can experiments give true data upon these points unless the following conditions are complied with:

I. A stock of known character, whose behavior, germinal constitution, variability, etc., have been determined for a series of generations and kept in strictest pedigreed line cultures. The stock for experiment must be clarified and reduced to a homogeneous condition as far as possible, and the presence of minor strains fully determined and eliminated.

2. It must be known what stages in the development of the germ cells, if any, are capable of being influenced, and how-by any force intended to be used later as a somatic modifier. Further, the behavior in inheritance of these germinal modifications, if any, must be known for several generations.

3. The somatic change must be induced at a time when the germ has been found to be not sensitive to the stimulus employed, so that opportunity may be provided whereby there will supposedly be accumulated in the modified soma that something, carrying the potentiality of reproducing the modifications which the soma has acquired and which are believed by many to become incorporated into the growing germ cells as part of their constitution. 
4. In any experiments four parallel series must be carried: (a) a parent stock from which at the start is derived the experimental stock; (b) the controlled stock reared parallel to the parent stock but under controlled conditions, (c) the experimental stock, which is a line culture subjected to conditions of experiment and continued throughout under the conditions of experiment, but from which is taken at intervals, and $(d)$ the test series, which are the progeny of modified stock returned to conditions of the control and parent, to test the constancy or reappearance of induced modifications.

5. All lines must be group cultures mated at random to obviate in the fullest possible manner any traces of selective effects; that is, to breed from pairs of selected extreme individuals might easily lead to the selective accumulation of germinal variations normal to the race, or the isolation of hitherto unrecognized pure lines, and thus give rise to false conclusions.

If these conditions are complied with in any series of experiments, the results will be an accurate answer to the problem-no matter whether brief or long-continued action be necessary to bring about the inheritance-because this may also be tested if the soma be modified at a time when the germ is not sensitive, and if this be repeated generation after generation the results obtained become more and more certain in their value as evidence for one or the other side of the controversy.

Furthermore, experimental procedure of this kind will at once give an answer to the question of the influence of the soma as an environment upon the germ cell. That is, by incident conditions it is easy to modify temporarily the physiological state of the soma and gain further knowledge 
concerning the influence of altered body states in producing germinal variations.

It will not do to dodge the issue as to experimental methods by the citation of experiments where these precautions have not been taken and say, What matters it, the end result is the same - a modification? True, a modification, inheritable, has resulted in so many series of experiments that there no longer are any doubts thereon. But that does not and cannot answer the important theoretical question because experiments have all too often not been properly oriented and guarded. In this there is a direct experimental proof of the main contention of Lamarck and C. Darwin, that incident conditions produce permanent modifications. Naturally, in order to be permanent, any departure from the normal must become a part of the germinal constitution-a process which Lamarck never attempted to explain, and of which C. Darwin offered only a formal explanation in his provisional hypothesis of pangenesis.

I have attempted to obtain what I considered reliable data upon this mooted point in the inheritance of somatic modifications, and one example of the results obtained when the procedure outlined has been followed may be given.

To determine whether coloration changes in the soma produced as the result

of changed environmental conditions are inherited, increased, or dropped in successive generations.

Conditions.- Temperature on the average $6^{\circ} \mathrm{C}$. and relative humidity ro per cent above that in nature, with other conditions natural. These conditions were planned to produce melanic tendencies in variation.

A pparatus.--Shown in diagram in Fig. 59.

The experiments in this series were conducted in the years 1900 to I904, and were carried through ten lineal generations. The conditions of temperature and moisture were as follows. 

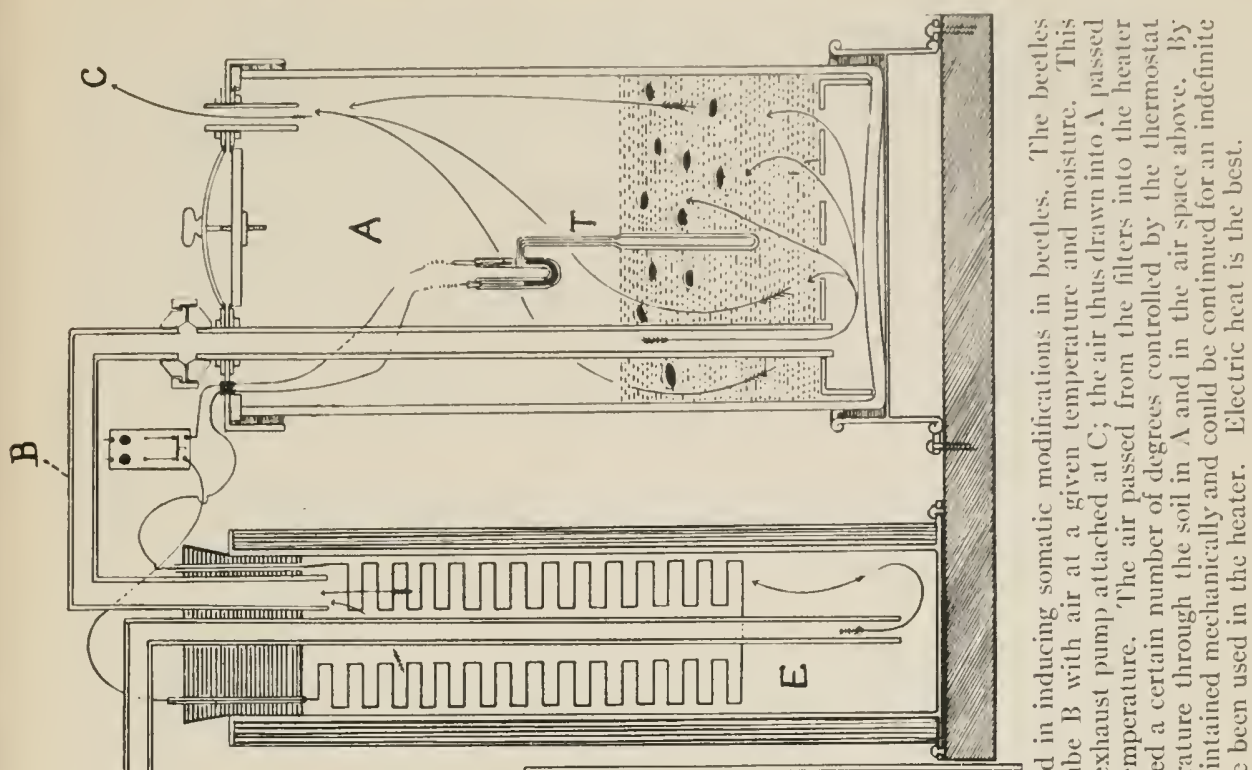

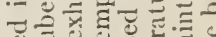
跣范范

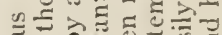

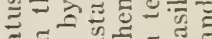

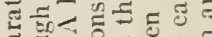

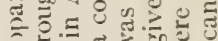

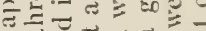

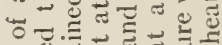

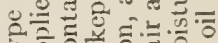
ट)

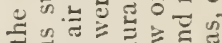

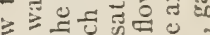

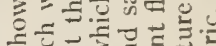

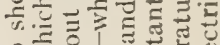

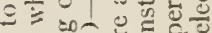

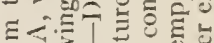
可山的的可 So. $\stackrel{0}{=}$

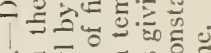

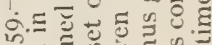

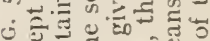

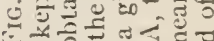
난

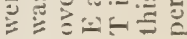


TABLE I

Temperature and Humidity Conditions

\begin{tabular}{|c|c|c|c|c|c|c|c|c|c|}
\hline & 7 A.M. & $\begin{array}{l}10 \\
\text { A.M. }\end{array}$ & I P.M. & 3 P.M. & 8 P.M. & $\begin{array}{l}\text { Maxi- } \\
\text { mum }\end{array}$ & $\begin{array}{l}\text { Mini- } \\
\text { mum }\end{array}$ & Average & $\begin{array}{l}\text { Devia- } \\
\text { tion from } \\
\text { Normal }\end{array}$ \\
\hline $\begin{array}{l}\text { Temperature, dry bulb } \\
\left.\text { ( }{ }^{\circ} \mathrm{C} .\right):\end{array}$ & & & & & & & & & \\
\hline In nature .......... & 19 & 22 & $3 I$ & 23 & 22 & 33 & I3 & 22.2 & o \\
\hline $\begin{array}{l}\text { In experiment } \\
\text { Percentage of relative } \\
\text { humidity: }\end{array}$ & 22 & 28 & $3 I$ & $3 \mathrm{I}$ & 23 & 40 & I9 & 28.4 & -6 \\
\hline In nature .......... & ${ }^{*}$ I00 & 65 & 50 & 55 & ${ }^{*} 100$ & 100 & 43 & 74 & o \\
\hline In experiment. & ${ }^{*}$ I00 & 85 & 60 & 75 & ${ }^{*} \mathrm{I} 00$ & 100 & 55 & 84 & $-I$ \\
\hline
\end{tabular}

* Dew.

In this series of experiments $2 \mathrm{I}$ per cent died in the larval stage and 9 per cent in the pupal, while 70 per cent appeared as imagines with the proper color modifications. Throughout. the whole series the greatest care was taken to prevent the conditions of experiment from having any possible influence upon the germ cells in their growth periods and during maturation and fertilization. This was accomplished by removing the adults to normal conditions during the period of germcell growth and fertilization, the fertilized eggs being returned as soon as laid to the conditions of experiment.

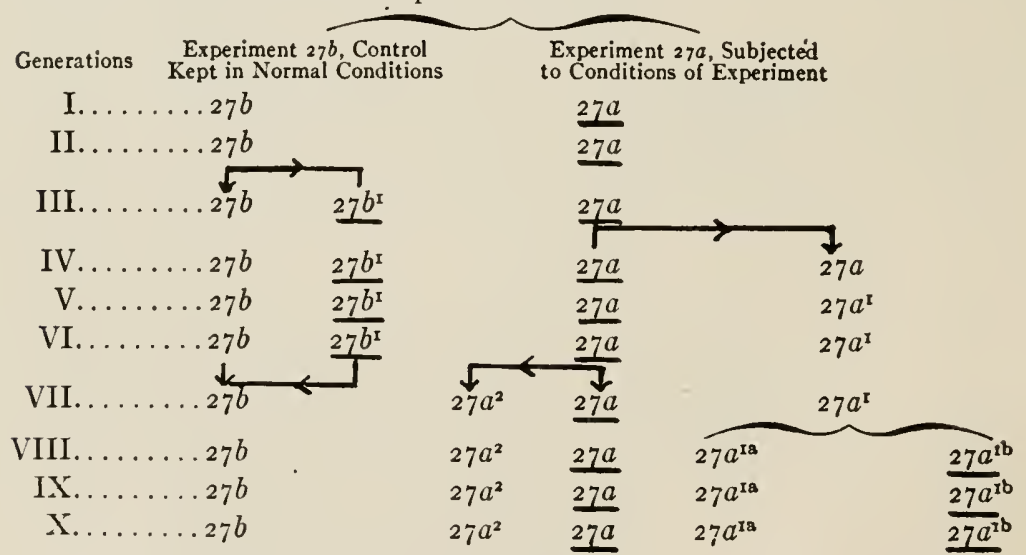

FIG. 60.-To show the different parts of an experiment, in which $L$. decemlineata was subjected to conditions which would modify the soma without modifying the germ plasm. The generations underscored were subjected to the conditions of the experiment; those not underscored were kept in natural surroundings. 
By this means the color changes induced by these experiments were known to be purely somatic modifications. Moreover, a control series, derived from the same parents, was kept under normal conditions as a check. During the series also several lots were taken from the experiments and placed for several generations in normal conditions, and were then returned to experiment; and likewise lots were taken from the control and placed in experiment; and subsequently returned to control. In this way a complete check was kept on the experiments. In Fig. 60 are represented the generations experimented upon and the proceedings followed with each.

The experiment was divided into two parts - the experiment proper $(27 a)$ and the control $(27 b)$. In $27 a$ the beetles were subjected to the conditions of experiment during ten lineal generations, with results shown in Fig. 6I. A maximum deviation in coloration was produced at once toward a melanic state from which there was no deviation either above or below in the succeeding generations. In the third generation of $27 a$ the progeny were divided into two lots of equal size, one of which was kept in the conditions of experimentation, and the other returned to natural conditions. This second lot, known as $27 a^{1}$, after being bred during four generations in normal surroundings, was further separated into two portions, one of which was still kept in normal conditions as $27 a^{12}$, while the other was returned to the conditions of experimentation as $27 a^{\text {tb }}$. When the beetles in $27 a^{x}$ were returned to normal surroundings, they at once resumed their natural characters and did not deviate therefrom during the four generations of $27 a$ and the three of $27 a^{\mathrm{Ia}}$, or seven in all. However, the effect upon $27 a^{\mathrm{lb}}$ of being returned to the conditions of experiment was an immediate return to the maximum melanic tendency before observed. From the sixth generation in $27 a$ another lot of beetles, $27 a^{2}$, were taken and reared in normal conditions, with the result that they also immediately reverted to the parental condition, and the same was true of $27 a^{3}$ in the ninth generation. In experiment $27 b$ there appears a slight oscillating variability which, however, is of no consequence. In the second generation $27 b$ was likewise separated into two lots of equal size, one of which, $27 b$, was retained as control, while the other, $27 b^{1}$, was placed in the conditions of experimentation for four generations, and later in the seventh generation returned to control with $27 b$. 
The effect upon $27 b^{\mathrm{r}}$ was an immediate production of the maximum melanic condition, which was retained throughout the four generations of experimentation, and lost only when $27 b^{\mathrm{x}}$ was returned to control.

In Experiment 27 there was no artificial selection, all imagines being allowed freedom to mate and breed as in nature; hence the only selective influences present were those exercised in the mating of the beetles and by the conditions of the experiment, which eliminated a small percentage.

From the data of this experiment the following conclusions are derived:

I. A deviation in an environmental complex at once causes the polygon of somatic variation and the modal class to shift as far from the normal as it can go under the given condition, and keeps them there until there is a return to the normal environmental complex, when the somatic variations also at once return to their normal state.

2. The color variations employed in experiment, which are purely somatic, are the direct result of a response to changed environmental conditions, in terms of increased or decreased activity in pigmentation. They may change as rapidly, as frequently, and in as many directions as the conditions producing them change, and they have no influence whatsoever upon the coloration of succeeding generations.

This experiment, one of the earliest in my series, illustrates fairly well the results obtained in several others carried out with the same end in view. In all, the result has been a uniform one, and entirely against the idea of transmission of somatic modifications. I have had many instances when I thought at first that a somatic transmission had taken place, but in all, further analysis and repetition clearly showed some defect in experimentation; and in no case have I been able to duplicate one of these occurrences, much less obtain experimental proof of somatic transmission in a repetition of the experiment.

It is unfortunate that so many of the published experimental investigations of this subject are open to diverse 
Modification of Germinal Constitution of Organisms $\mathrm{I}_{3}$

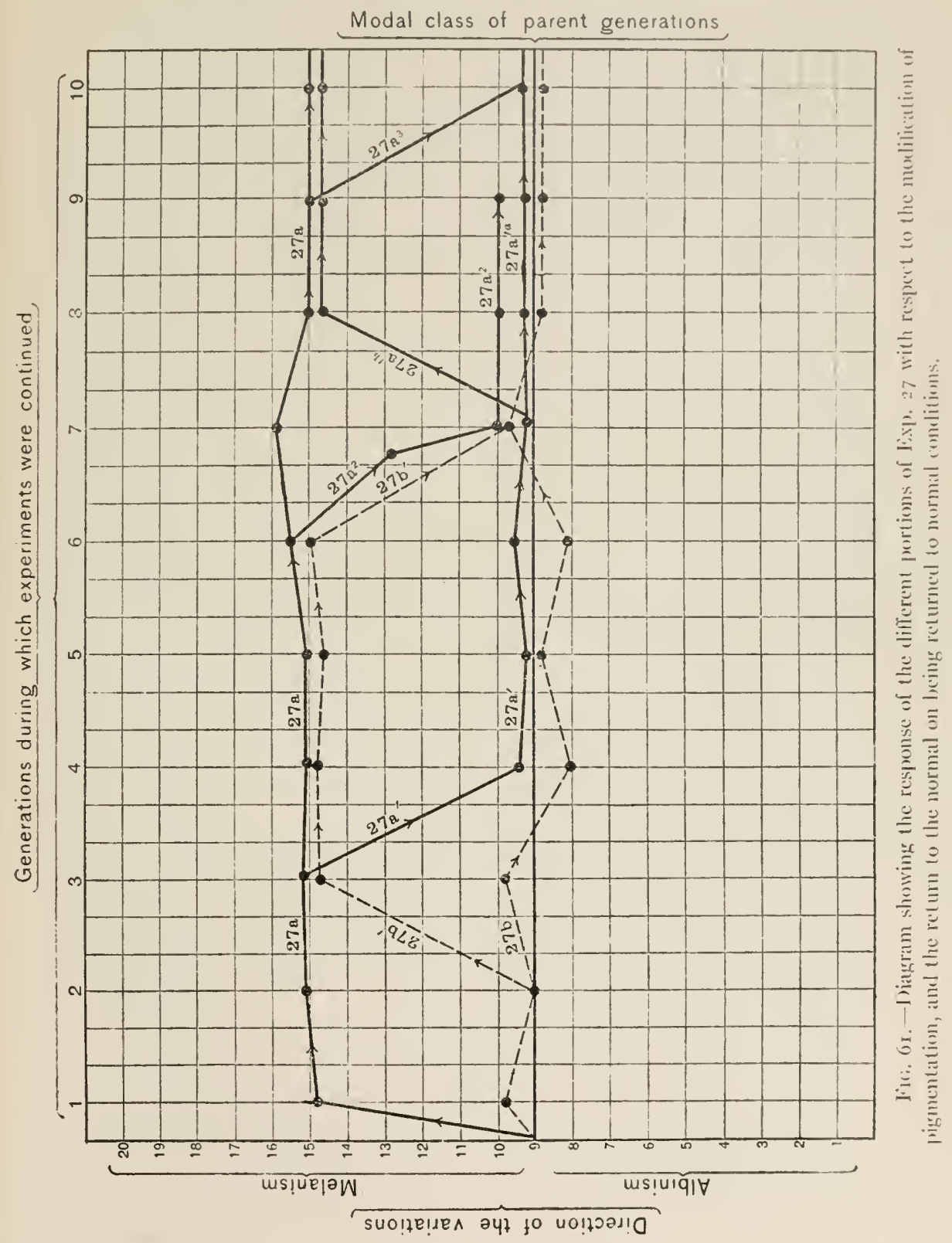


interpretation and are not elucidations of the problems. The main reason why this is so is that the materials that have been used and the methods of experimentation have not been properly guarded. Thus, for example, the recent results of Kammerer with various amphibians and Lacerta, Woltereck's investigations upon Daphnia, Zederbauer's experiments with Bursa, Sumner's with mice, as well as all of the older experiments, admit of "interpretation" from either point of view. Thus Semon "interprets" all of these and many more besides as showing the strength of the neoLamarckian position at the present time. A neo-Darwinian could make an equally good case of the same data. At present the conclusive evidence from Castle's transplantation of ovaries in guinea-pigs, Davenport's negative results with poultry, and experiments like those with color in Leptinotarsa have all given exactly the expected result without qualifications. MacDougal, in discussing some of these problems, says: "The time has now arrived when the claimants for neo-Lamarckianism and all of its conclusions must show cause for its further consideration, or else allow it to drop from the position of being seriously taken as a method of evolutionary advance."

With this most biologists will at present agree, but unfortunately, from time to time, some careless experimenter with more partisan enthusiasm than judgment or experimental acumen will come forward with conclusions derived from experiments wherein the most elementary essentials of genetic research are ignored and reassert the transmission of somatic changes. Present experimental evidence, where critical, clearly indicates the increasing doubtfulness of the validity of the hypothesis of somatic transmission. 


\section{THE DIRECT MODIFICATION OF THE GERM PLASM}

Since there are the best of reasons for the conclusion that there is conditioned in the germ plasm the basis which determines the presence and manifestations of organic characteristics which are permanent in the race and in evolution; and in that there are equally good reasons for a tenacious adherence to the idea that all variations which are productive of evolutionary changes arise primarily in the germ and appear secondarily in the soma, it follows that any and all methods whereby changes are produced in the germinal material are of paramount interest. Modifications in this germinal material are the basis of permanent departures from the racial mean, and at present the methods of production and cause of germinal variations are of great practical interest and value as well as of theoretical importance. Germinal variations have been suggested to arise by five main methods.

The direct action of external forces was the first to be suggested and is generally admitted to be an effective cause in the production of germinal variations. This was a mode of modification suggested by Buffon and Erasmus Darwin, later elaborated by Lamarck, and made the basis of his theory of evolution without any consideration whatsoever as to whether the variations were somatic or germinal. A half-century later variations which were supposed to hare originated through the action of external conditions provided in the main the array of individual differences upon which Darwin founded his theory of the "origin of species," by means of natural selection.

Arising from the work of Darwin, and accentuated by the neo-Darwinians, is the idea of the production of variations 
through selection, but how selection is conceived to produce these results depends very largely upon an endless array of unproven neo-Darwinian assumptions.

Hybridization is known to be productive of germinal variations, and in domesticated organisms a considerable number of useful forms have thus arisen. Frequently, however, these commercial hybrids, appearing to be constant, are only first generation hybrids indefinitely perpetuated by cuttings, as are many kinds of oranges, apples, grapes, etc., and most of these, if allowed to reproduce sexually, would in subsequent generations break up into the component types out of which they were built. There is evidence, however, to warrant the assumption that hybridization does result in the development of permanent modifications which are new to the strain in which they arise, and which persist indefinitely. Moreover, hybridization is a potent means of creating new and diverse combinations of existing qualities and attributes, which may account for no small portion of the "species" in nature, as well as in domestication. To what extent hybridization is a source of germinal variations in nature is undetermined, and this condition is largely due to the persistence of the dogmatism that hybridization is of rare occurrence, is abhorrent to species in nature, and is really a product of domestication and the supposed loss of specific integrity and chastity induced by man and cultivation. Statements of this kind, however, are entirely a-priori orthodox prejudices without foundation in fact. Recent work, especially by botanists, shows a considerable and increasing array of hybridizations occurring in nature, for example, in violets, which exhibit an abundance of crossings, with many resulting hybrids. Moreover, this condition is by no means limited to 
violets or plants, but is coming to be recognized as common in nature.

The production of germinal variations occurs by combining slightly different conditions of the same attribute in the zy'gote (fertilized egg). 'This process, amphimixis, commonly' adranced by neo-Darwinians, has at present almost no evidence in support of the theory that departures beyond the normal range of variation can be produced thereby, but it is at least a conceivable method by which variations might well arise, and is open to direct experimental investigation.

The origin and development of variations through the operation of orthogenesis, a name descriptive of a condition, but personified to represent the agencies productive of the condition observed. It is conceivable that changes started in one direction or another may continue in that direction on the basis of the operation of the law of inertia, in a uniform direction and at a uniform velocity until they reach limits imposed by the physical nature of the part or of the organism in which they arise, or until they reach a stage of development where they become of selective value, and may be accelerated, retarded, or turned in other directions.

In all of these possible modes of origin of germinal variations two groups of factors are always involved: first, the physical constitution of the material, with its array of qualities, attributes, and conditions, which is always the genetic product of an immense series of antecedent stages; second, incident forces from without the germinal material. These two groups of factors sustain definite and fundamental relations to each other, and the effort to understand the relations between these two fundamental groups has stimulated much of the investigation of the last decade. The physical or gametic constitution is the constant, and 
the external conditions are the variable in the complex, and elimination or understanding of the most obvious variable is the first step in the study of gametic constitution and modification. Concretely, then, what is the rôle of external factors in the production of germinal variations? Satisfactory evidence as to the rôle of external factors can be obtained only through careful experiments. These may be either experiments under laboratory conditions, or experiments in nature, and, if possible, both should be carried on at the same time upon the same materials.

\section{A. The Idea of Sudden Transmutation in the Germinal Material}

Succeeding Darwin, there arose a group of followers - the neo-Darwinians. Possessing all the attributes of followers, unable to grasp the breadth of view of their master, and seeing but a particular phase of his general teachings, they endeavored to raise that to undue prominence and make it a universal motive force in the evolution of organisms.

The Neo-Darwinians in the last quarter of the nineteenth century, under the leadership of such men as Weismann, created what Eimer has termed the "principle of omnipotent natural selection." It was attempted to establish purposeful selection as the sole efficient cause of variation and evolution in organisms, and in the effort, Weismann went so far as to place the selective process, not in the outside world, but in a microcosm within the germ plasm, making it in every way incapable of investigation, impossible of observation, and all-inclusive.

Few today attach much importance to Weismann's "germinal selection," and the Weismannian theory remains in biological history one of those curious ideas comparable 
to the quadrille of the centrosomes. The last of the curious hypotheses developed by the neo-1)arwinians is that weird phrase of biology which took its rise from the work of Bates, Muiller, and Trimen, and which has been developed into the present theory of mimicry. Its supporters would have us believe that much transmutation is based upon a mimetic principle aided by the subsidiary principle of recognition marks. Here utilitarian variation and purposeful selection run riot and produce in every case a definite end, a protected form. Naturally, these curious and thoroughly uncritical ideas, current among the neo-Darwinians, are their own answer.

At present the neo-Darwinian concepts offer nothing that is of use as a working hypothesis in the further investigation of evolution, nor any logical ground for observation and induction in nature. The neo-Darwinian situation, and, also, the neo-Lamarckian are in reality two of those common developments which arise in every line of human thought-intellectual culs-de-sac.

More as a protest against the neo-Darwinian situation than for any other reason, there arose, simultaneously, in England, on the Continent, and in America, the modern saltationist school. Thoroughly bored with the repetition by the neo-Darwinians of the same old facts sung to the same old tune, Bateson in England, DeVries on the Continent, and others, determined to find an outlet, and all, I think, obtained their original inspiration from the recognition by Darwin that in many species variations repeatedly occur which stand apart from the rest of the population, and frequently are prepotent when bred back to the parent stock. Further, Darwin in his Origin of Species and The Variations of Animals and Plants under Domestication cites 
many instances in which there is good reason for believing that domesticated varieties of pigeons, birds, and cattle, and many plants, have arisen by the sporting process.

Most biologists have insisted upon retaining that cherished dogmatism of the seventeenth and eighteenth centuries that there must be discontinuity between species, and the idea seemed plausible that if discontinuity existed between species when they were finished, there was no a-priori reason why it could not have arisen at the start. Therefore, Bateson, in the latter part of the nineteenth century, gathered what data existed in the literature on sports and discontinuous variations, in the effort to find an outlet from the cul-de-sac into which neo-Darwinianism had led English biologists.

At about the same time DeVries in Holland became convinced from a somewhat different point of view that a similar process must be operative in the production of species in nature. DeVries sought, therefore, to find in nature plants which exhibited the kind of variation that he conceived of as being the basis of transmutation, and finally discovered that Oenothera Lamarckiana seemed to be undergoing exactly the sort of process which he hoped to find.

Others became convinced that there were possibilities in this direction, and as a result there is at present a welldeveloped school of saltationists whose central idea is that progressive and efficient steps in transmutation take place through sudden, steplike variations, producing, as DeVries asserts, something quite new each time.

Directly associated with the development of this idea, and contributing much to its development, was the rediscovery of Mendel's paper on the "Behavior of Hybrid 
Peas," which gave impetus to investigations that strengthened and extended the saltationist conception.

It would be rash indeed to deny that there are "sports" in the Darwinian sense, and DeVries' "mutations" are asserted by some to be but the same kind of variations with a new name, but the fact of the occurrence of sudden variations is established beyond doubt. However, next to nothing is known concerning the rise and behavior of these sports and the part they play in the transmutation of organisms in nature, and it may be wisest to suspend judgment as to the relative importance of saltation as a method of evolution. The saltation conception, however, considered in its broadest sense, has decided advantages over the neoDarwinian and neo-Lamarckian positions, because it is directly open to experimental study and does serve as a fairly logical and workable hypothesis for investigation. In its broadest aspects it is in no way like Weismann's theory, although DeVries has endeavored to give it a position not unlike that of germinal selection by placing all essential processes in the hypothetical pangenes. Out of this situation perhaps the greatest advance that has been produced is the revival of interest in bionomic investigations and the clearing of the mist from many questions, even though the questions have not been fully answered. Regardless of what the future may have in store, the saltationist school has rendered biology a very real and lasting service in arousing new enthusiasm for the experimental study of evolution problems and in breaking away from neo-Darwinianism, neo-Lamarckianism, and orthogenesis, whose deadly chants were slowly but surely lulling into complacent inactivity the greatest heritage from Darwin-the experimental study of evolution problems. 
The saltationist school, aside from a considerable number of clearly formulated questions, has very clearly raised the issue as to whether changes in the constitution of the germinal material are accomplished by the slow quantitative accumulation of useful variations, or take place by sudden steps, appearing with discontinuity in the end result. Much experience with this method of quantitative accumulation had given adequate reason to distrust it as a particularly potent means of inducing experimental change, and refuge not infrequently had been sought in the soul-satisfying myths of germinal selection, orthogenesis, isolation, growth force, bathmic force, and many other intricately contrived and all inclusive hypotheses, but all were found utterly useless for actual experimental investigation and analysis, such as is demanded in physical and chemical science.

In seeking for an outlet from the culs-de-sac of evolutionary science as it existed in the last quarter of the nineteenth century, DeVries concluded that change, per saltum, was quite as liable to be a real method of transmutation and sought to put it to a test by seeking in nature for species of plants that were undergoing this kind of change if such existed. Darwin had already noted the frequent occurrence of large sudden departures in both plants and animals, but was of the opinion that both large, sudden, and small fluctuations were operative in transmutation phenomena.

The discovery of $O$. Lamarckiana (Fig. 62) in an abandoned field near Hilversum in Holland, into which it had escaped from a near-by park, provided most favorable material for DeVries' further study. Here it grew in quantity with two apparently newly arisen derivative forms, $O$. laevifolia and $O$. brevistylis. Plants from this waste land were taken into the botanic gardens of Amsterdam 


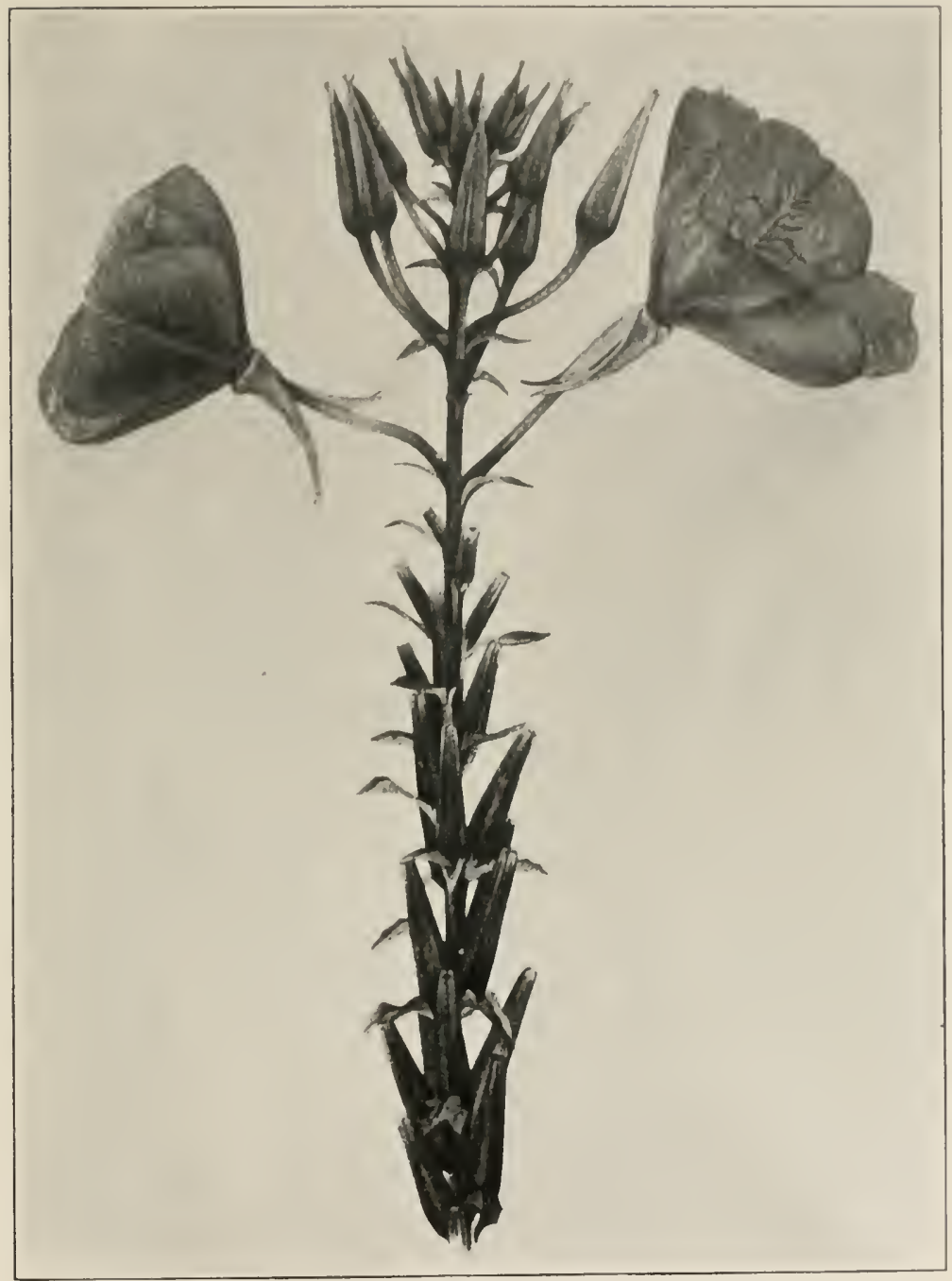

FIG. 62. - Oenothera Lamarckiana, the original type of plant used by Deliries in his experiments. This is the stock from Hilversum, from which arose in successive generations a series of new forms by sudden jumps. From Delries. 
and there gave rise to several new types during the years of DeVries' observation. Some of these arose in the experiments but once, as for example, O. gigas (Fig. 63), a tall, robust form with large flower, the finest of all the new types. It appeared in I 895 and was one out of about I 4,000 plants, but was not the only new type to appear in the crop of that year. Six others were found: O. albida, of which there were fifteen examples (Fig. 64); O. oblonga (Fig. 65), of which I76 specimens appeared; $O$. rubrinervis, eight specimens; O. scintillans (Fig. 66), one specimen. This year gave the greatest number of new forms of any, although other years (I896, I897) gave all but the 0 . gigas.

A good idea of the real differences existing between these derivative forms and the parent plants is given in Fig. 67, where the plants are shown growing side by side.

In Fig. 68 is given in condensed form the line of descent and the appearance of the derivative forms from year to year.

If all be granted that is claimed for the separateness of these types, and admitting also that they are absolutely constant in type and in heredity, there still remains one striking difference between these DeVriesian "mutations" and the "sports," "saltations," etc., of other writers: namely, the mutations occurred in numbers in every generation for a considerable period, through several consecutive generations; the sports of Darwin appear but once, rarely, and not successively. Upon the curious findings in O. Lamarckiana, DeVries has built the hypothesis of a premutation period in which the germ plasm was elaborating new pangenes which, when the pangenes reached a certain point, broke out into visible manifestation as mutants, and he further supposed that after a time the new 


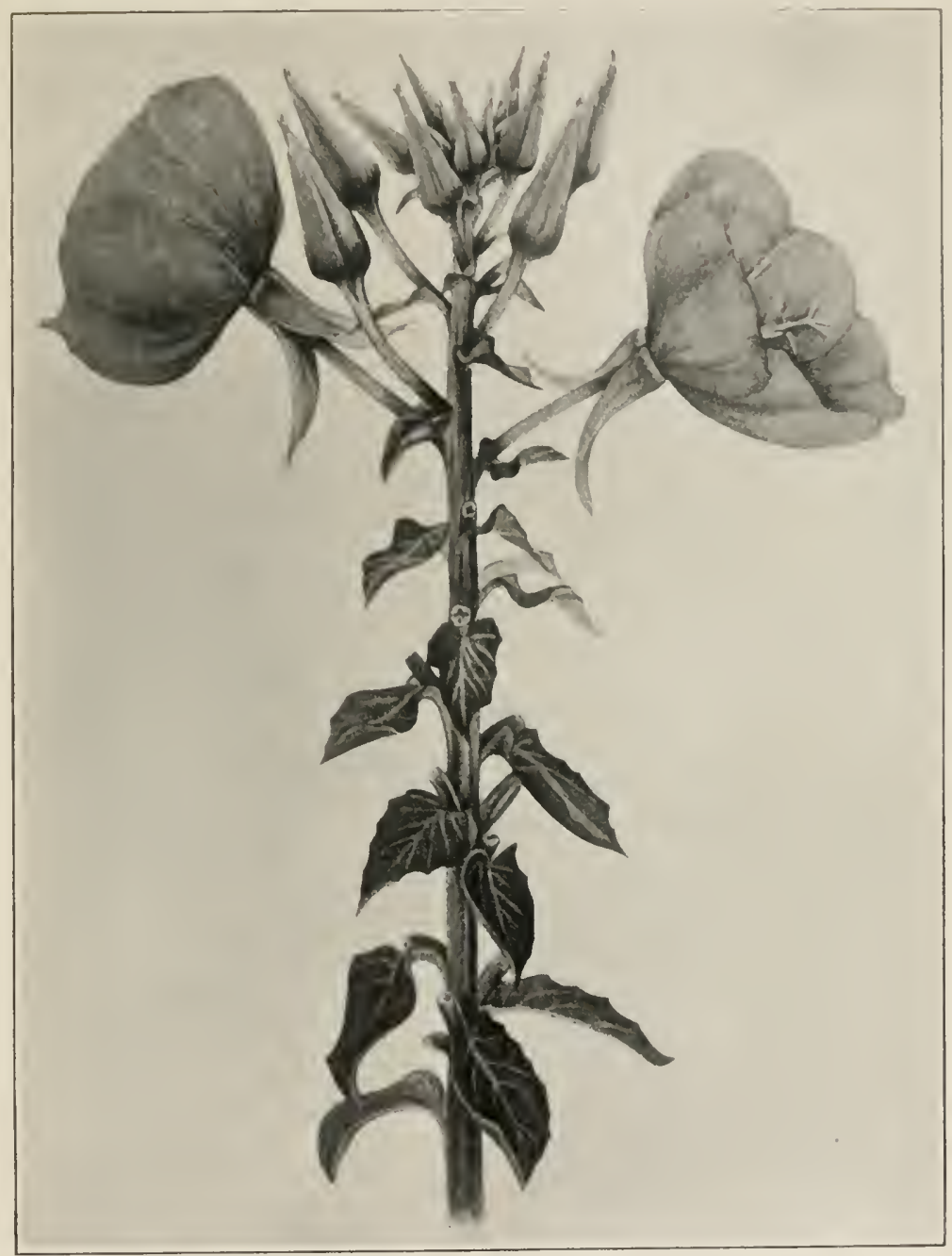

FIG. 63.-Oenothera gigas, a mutant of Oenothera Lamarckiana. This form arose but once in DeVries' cultures (in 1895 ), out of a culture of 14,000 seedlings. From it has arisen a strong race now cultivated in many gardens in Europe and America. 


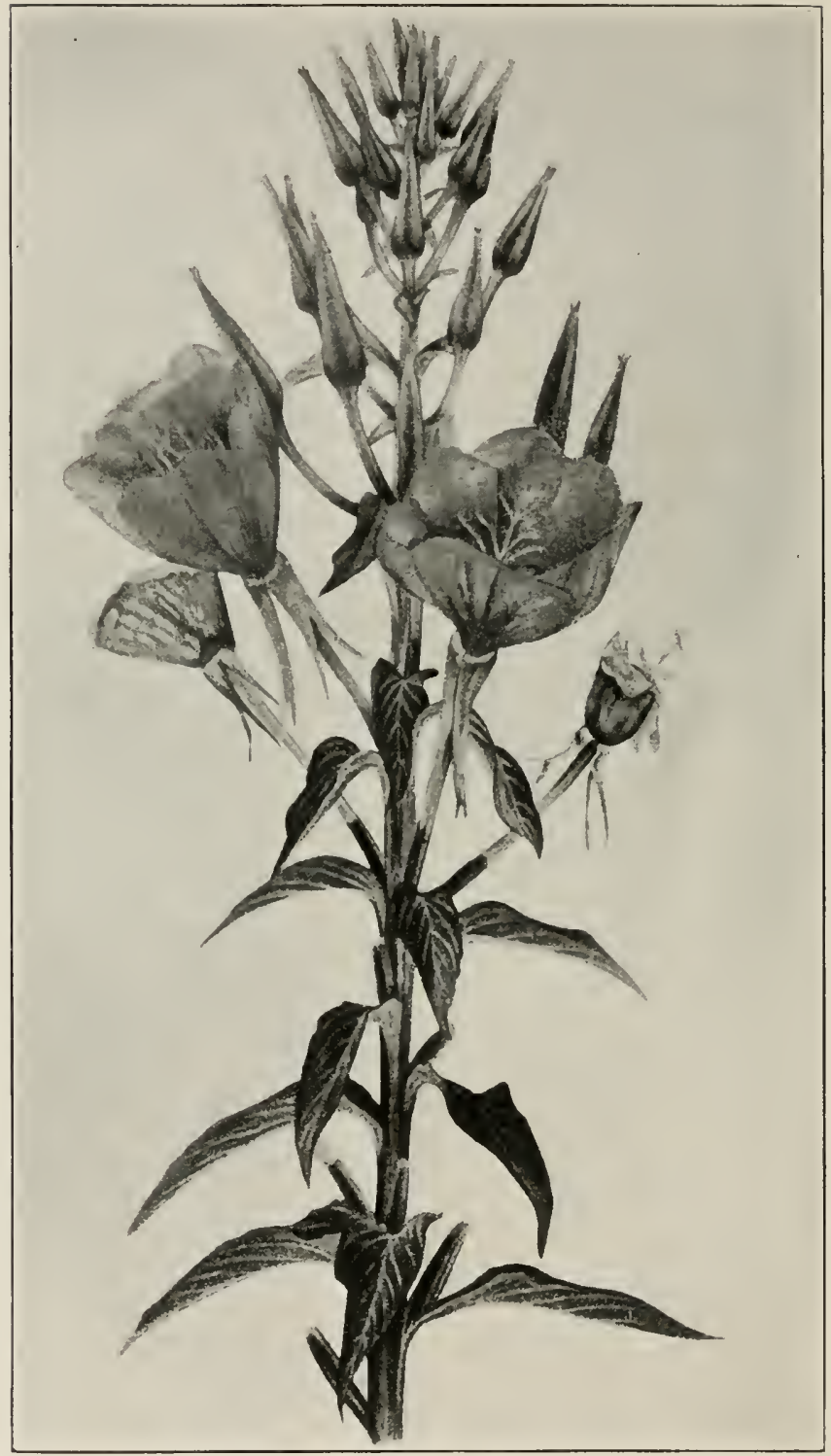

FIG. 64.-Oenothera albida. Another type which has arisen from Oenothera Lamarckiana, occurring with considerable frequency in successive years. 


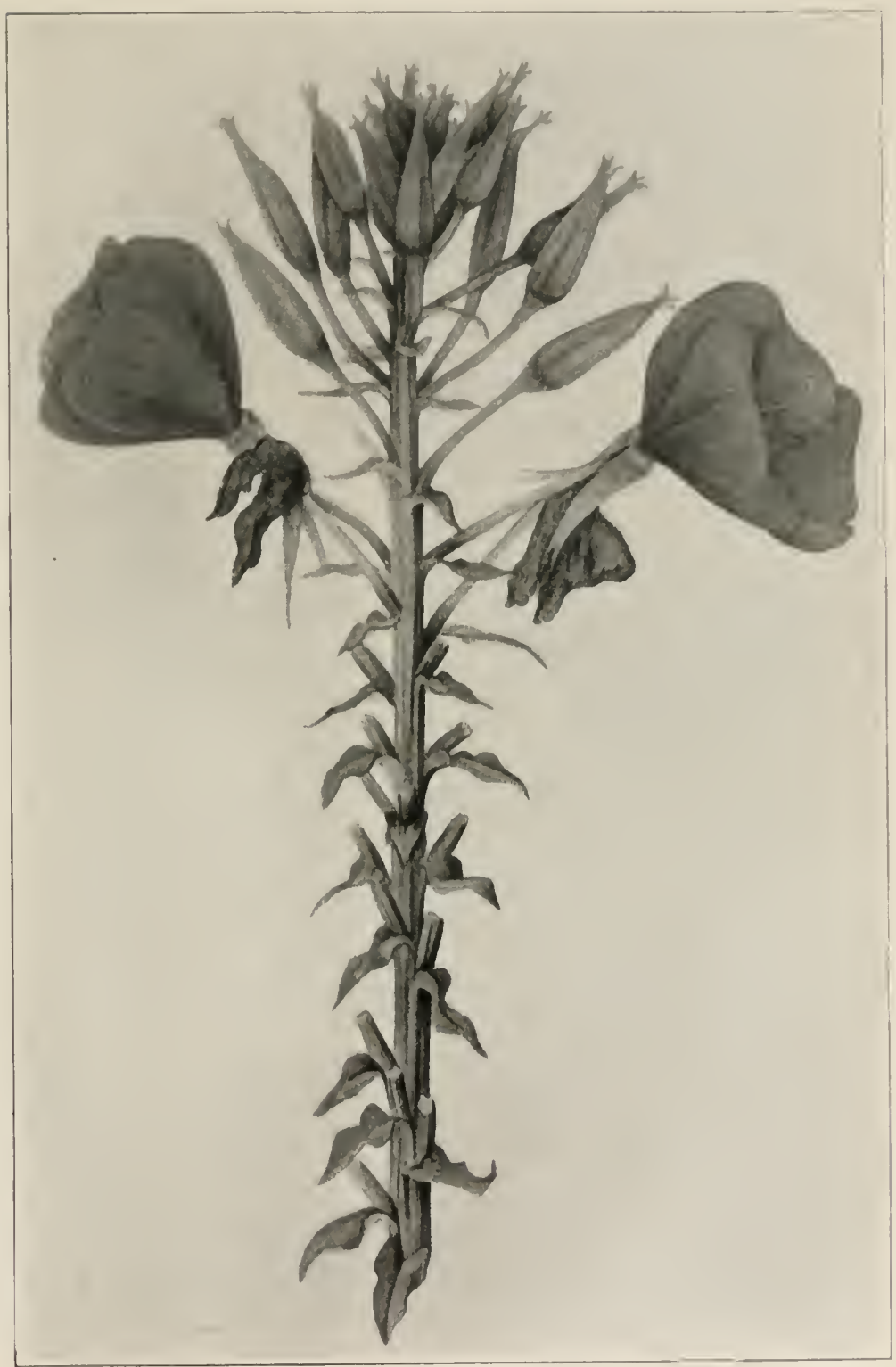

Fig. 65.-Oenothera oblonga. A type which has arisen from Oenothera Lamarckiana. 


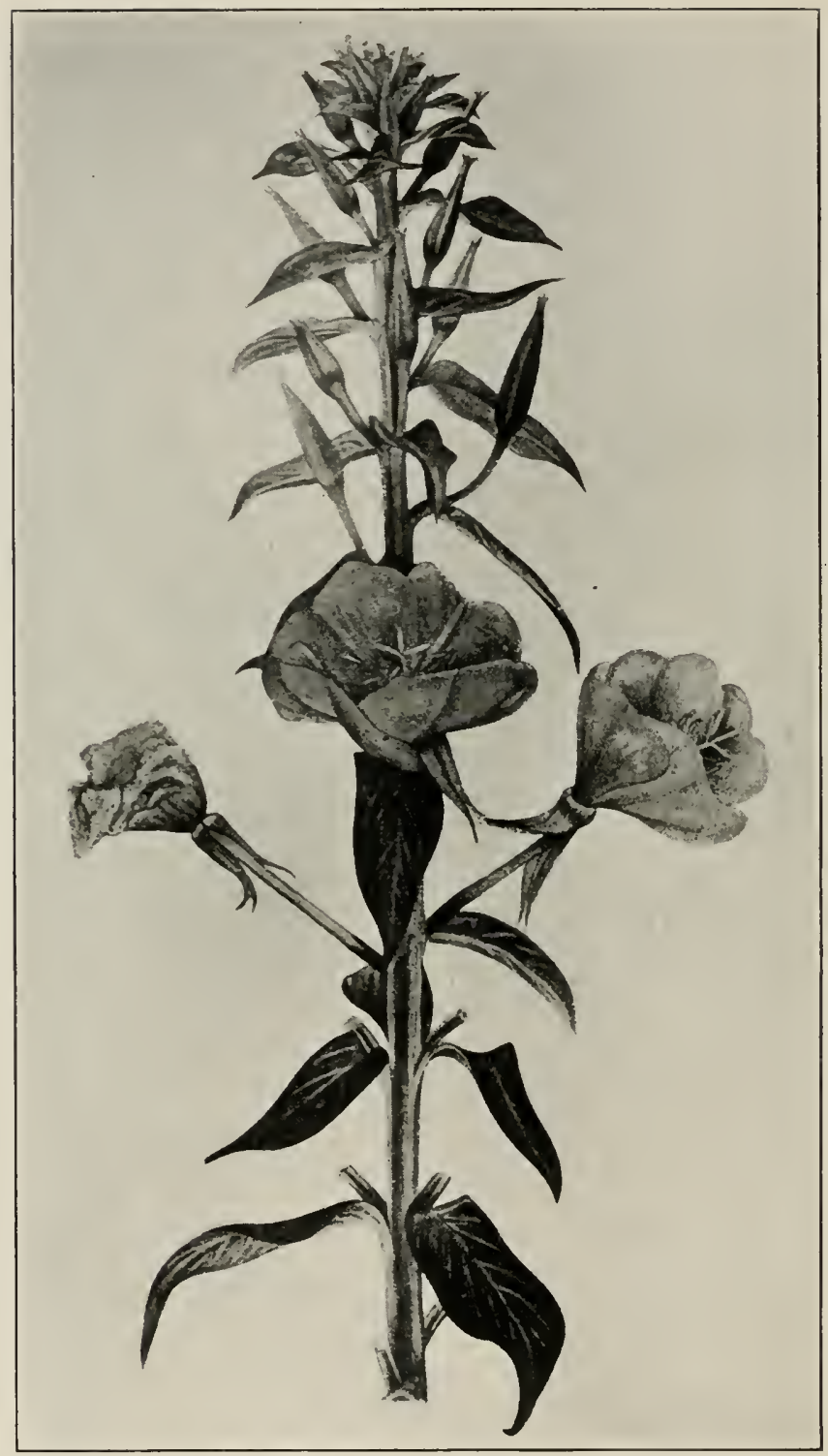

Fig. 66.-Oenothera scintillans. A rather rare mutant of Oenothera Lamarckiana. 


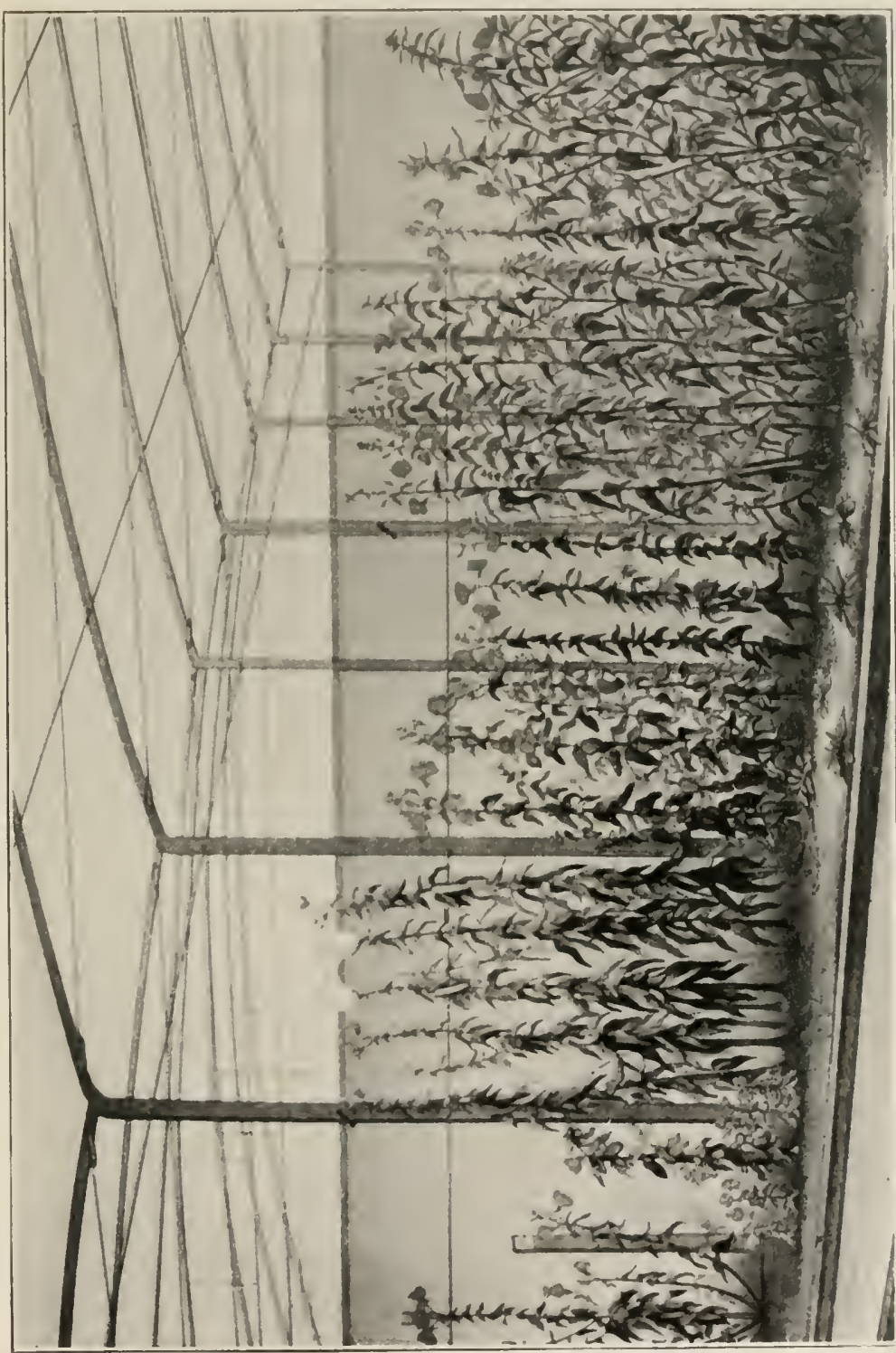

$\equiv$

范

赵

-

三

茫

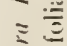

离

는

- $\quad$

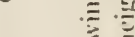

方

急

ริ气

$\Xi$

.00

華

$\therefore$

章 
pangenes would cease to be produced, or at any rate that mutation would cease and the parent species go on as before.

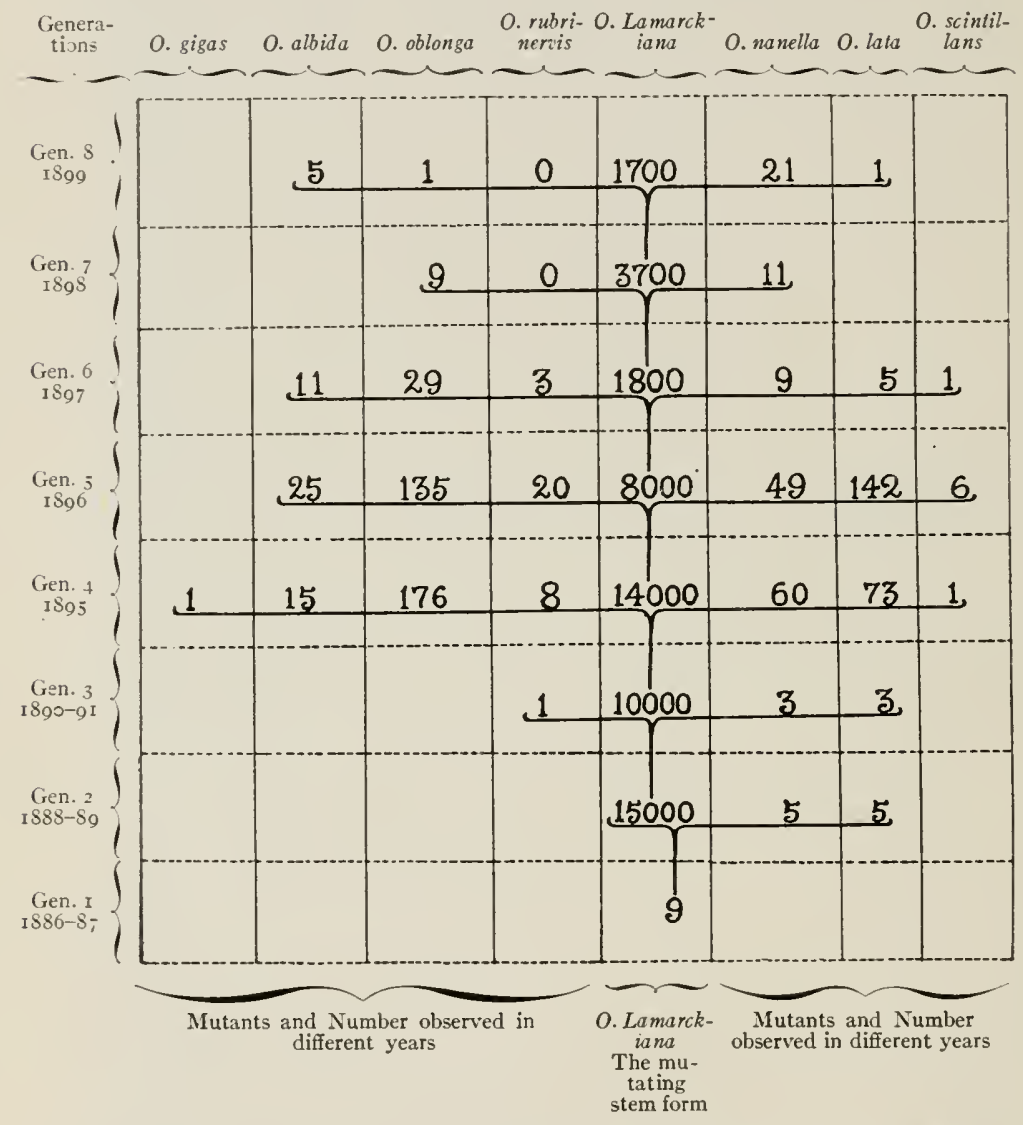

FIG. 68.-Diagram showing in condensed form the genealogy of the Oenothera Lamarckiana family and its various mutants during successive years. The numbers under each type represent the number of new types observed in each year.

As far as causes were concerned, DeVries had little to offer except the suggestion that the cause was ultimately probably an external one. 
There is not the least doubt as to the behavior of $O$. Lamarckiana and the appearance of the "mutants," and it appeared to many that there was a good chance of producing "mutating races" by external forces acting upon the germ of the parent race. The last decade has produced a deal of evidence that external forces can produce germinal changes, but these are in all instances immediate and final. New, divergent types, more or less separated from the parent, have appeared, but in none are there subsequent mutations.

I had been at work upon this problem and had found and reared sports of Leptinotarsa decemlineata as early as IS93, and on the appearance of DeVries' work I began in a systematic way to try to produce mutating races by the use of external forces. I have thus far positively failed to produce a mutating race by these agencies, although I have been able to get changes in profusion, some of which I shall describe later.

In Igo I I tried to produce a mutating race by crossing L. decemlineata, L. juncta, and L. pallida, with the idea that perhaps the interbreeding and combination of the chief characters of the three into a hybrid complex would produce a type which under changed conditions of growth and development, or of changed or adverse environment, would give the mutation behavior of $O$. Lamarckiana. The early experiments were just beginning to show promise of interesting results when they were brought to an end. As subsequent results have shorn, this was a good working hypothesis but these first experiments would not have led to the results wanted. In I902 Bateson suggested that the mutation phenomena in Oenothera was possibly akin to a Mendelian splitting of a hybrid type. 
From the year I904 onward I have been able to carry out a number of suggestive experiments in the further effort to produce a mutating race experimentally.

EXPERIMENTS IN THE SYNTHESIS OF A MUTATING STEM RACE

An extensive set of these experiments has been in progress for some years, some of which have now developed far enough to allow of rather definite statements. The method employed has been to take species derived from nature from some restricted locality, to keep close watch upon what goes on in this locality, and also to analyze the composition of the species from this locality by cultures in the laboratory. In this way, stocks of known character are obtained from experiment, and also natural stocks whose attributes are well known are developed in the type localities. In the experiments in synthesis either pedigreed stocks from the laboratory, or the stocks from nature, or both, are placed in nature upon their food plant in isolated localities, or in large cages, and allowed to breed as if the introduction were a natural one.

In I904, an isolated area of about an acre upon the southern slope of a barranca, near Cuernavaca, was planted with food plants, upon which both $L$. signaticollis and $L$. undecimlineata would feed. In July, I904, this spot was stocked with a culture of 2 IO specimens of L. signaticollis, from a standard location about a mile and a half distant, and 354 specimens of L. undecimlineata, obtained at El Hule, on the banks of the Rio Papaloapan. The groups were equally divided between the sexes, were young and vigorous, immediately began breeding, and intercrossed freely. Under experimental conditions these forms cross freely in 
both directions, but out of them no new characters come as the result of ordinary crossing.

In the first generation of this colony there was an abundance of individuals of both sexes of the signaticollis type, and of the undecimlineata type, and of a highly variable intermediate hybrid type. A census was made of the population on August I 4 to I 7 , with the following results:

$\begin{array}{ccc}\text { Signaticollis Type } & \text { Mid-Type } & \text { Undecimlineala Type } \\ 4,5 \mathrm{IS} & \mathrm{II}, 744 & 5,09 \mathrm{I}\end{array}$

In this experiment, it was, of course, impossible to tell from inspection whether the signaticollis individuals were pure signaticollis, or pure signaticollis and a hybrid with the signaticollis dominant, and the same was true with respect to the undecimlineata. All of the beetles entered into hibernation during the latter part of August and early in September, 1904. The food plants survived the long, hard, dry season and came up in the spring of 1905 in abundance, and in June, 1905, individuals of all three types emerged and were found to be interbreeding freely. A census made of the individuals which emerged late in June gave the following results:

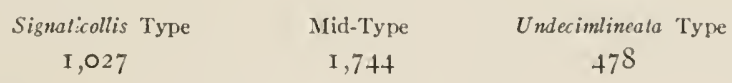

which clearly indicate that through some cause the hibernating conditions of the location were favorable for signaticollis, but decidedly unfavorable for the undecimlineata and for the intermediate hybrid type. These individuals were allowed to interbreed freely and produced a numerous progeny, in which the larvae were of four different types: white without spots, white with spots, yellow without spots, yellow with spots. The second generation emerged from the middle to the end of July, 1905, and showed a 
huge preponderance of the signaticollis type. The census of a random sample taken the last week in July gave the following count:

$$
\begin{gathered}
\text { Signaticollis Type } \\
\mathrm{I}, 244
\end{gathered}
$$$$
\text { Mid-Type }
$$

Undecimlineata Type

367

These individuals were not removed from the colony; the census of the sample was made, the individuals put back, and the colony allowed to encounter the conditions and behavior which it would meet in a state of nature. Nine pairs, taken at random, of the undecimlineata type were bred out as pedigreed cultures during August and part of September, 1905, and gave uniformly an undecimlineata progeny. Seven pairs of the signaticollis type, which were bred out, gave uniformly a signaticollis progeny, and out of five other pairs there appeared individuals of the mid-type and of the undecimlineata type, showing that some of the signaticollis type were hybrid in character. Six pairs of the mid-type were also bred out as pedigreed stock, and showed themselves to be in every case hybrid. The third generation was produced in August and early September, 1905. In this the larvae were of the same four classes, but showed a huge preponderance of yellow larvae $(y l S)$. A count made late in August, when perhaps the bulk of the larvae had entered into pupation, gave the following results:

$\begin{array}{lrcr}\text { Whs } & \text { WhS } & \text { yiS } & \text { I's } \\ 205 & 227 & 849 & 32 \mathbf{I}\end{array}$

The adults of Generation III emerged early in September; a census made about the middle of September gave the following: Signaticollis Type
\[ 2,45^{2} \]
Mid-Type

S27
Undecimlineata Type 2 I $S$ 
showing again a marked decrease in the undecimlineata form, a lesser decrease in the intermediate hybrid type, and a much greater relative increase in the signaticollis type. These hibernated during the winter of $1905^{-6}$ and emerged in June, I906. They were allowed to interbreed freely. The population was not seen at the time of emergence, but in the fourth generation it was observed in July, 1906, and the undecimlineata type and the mid-type were nearly absent. The census made at this time, when the first generation of the year was apparently at its height, gave the following results:

$$
\begin{gathered}
\text { Signaticollis Type } \\
3,275
\end{gathered}
$$$$
\text { Mid-Type }
$$

Undecimlineara Type

45

7

These then inbred and the colony was next seen in September at about the middle of the month, when the census of the individuals in the colony was as follows in Generation $\mathrm{V}$ :

Signaticollis Type

I. 823
Mid-Type

6
Undecimlineala Type

○

These hibernated during the winter of 1906-7 and emerged in June, 1907, reproduced at once, and gave an abundant progeny which emerged as Generation VI between the Ioth and $25^{\text {th }}$ of July. These when seriated gave the following results:

Signaticollis Type

2,255
Mid-Type

2

Undecimlineala Type

$\circ$

The second generation of 1907 emerged late in August and early in September, and of this generation the undecimlincata type was entirely absent, and the mid-type practically so. These hibernated and when seen in the spring of I 908 only the signaticollis type emerged. Both generations of I 908 and both generations of r 909 have developed the presence of the signaticollis type only. 
In rgo8 individuals from this location were brought to Chicago and carried as pedigreed cultures in the laboratory. They have shown a complete gametic purity as far as could be determined and none have been detected which were hybrid in character. In this colony, isolated in its location, through some process or other in hybridization or perhaps by selective factors, signaticollis has completely subjected and eliminated undecimlineata. Inasmuch as L. undecimlineata, when protected from crossing, lives well at Cuernavaca, and the selective action is very low, I am of the opinion that the swamping of undecimlineata is due to some process of hybridization. This opinion is fully justified by experiments conducted in cages which eliminate selective factors.

Another experiment was begun in 1905 , when one hundred individuals were taken from the standard colony of $L$. signaticollis at Cuernavaca, and, with an equal number of L. undecimlineata, from El Hule, were planted upon a vigorous growth of their food plants in a clearing made in the Foot Hill Rain Forest, in the Paraiso district, not far from Ojos de Agua, in the Canton of Zongolica. They were observed to intercross freely, but there was a preponderance of undecimlineata-like forms, with a few intermediates, and only small numbers of the signaticollis type in the first generation. The census made of the first hybrid generation was as follows:

Signaticollis Type

o
Mid-Type

56
Undecimlineata Type

I, $3+2$

A third generation was produced in late November, and in that generation there were no signaticollis forms visible; there were only a few of the hybrid intermediate type, and 
these all closely approximated the undecimlineala form. The census obtained late in Norember was:

Signaticollis Type

O
Mid-Type

I I
Undecimlineala Type

I. 132

In I906, I907, and I908 these cultures were allowed to shift for themselves, and the food plants were nearly swamped by the immigration into the glade of plants from the surrounding rain forest; in fact, the whole culture was allowed to engage in a most desperate struggle for its existence. As far as the beetles were concerned, this was simply a struggle for food. In I9oS-9 the inroads which had been made by other plants had so reduced the number of Solanums that the food supply was inadequate. During these years, however, no trace of the signaticollis type had ever appeared. In Igos, material of the undecimlineala type was taken from this culture to Chicago, and there subjected to the tests of pedigree analysis, but without any trace of the signaticollis form appearing. In both experiments, however, at Praesidio and at Cuernavaca, the resulting materials were different in gametic makeup from the original species. Superficially, these stocks could not be told from the natural species, but when used as the basis of experiment under control conditions, it was found that there resulted a difference in the behavior of the subsequent hybrid generations, clearly indicating a change in the gametic constitution of these groups of individuals.

A series of experiments, more conclusive and under better conditions, has been carried on, using three species: $L$. decemlineata, L. oblongata, and L. multitaeniata. Of these, in nature, L. decemlineata is limited solely to the 
United States and southern Canada; L. multitaeniata entirely to the southern portion of the plateau of Mexico, and $L$. oblongata to the Balsas Valley and the OaxacaGuerrero Highlands. These species intercross freely under experimental conditions and represent the following contrasting characters for consideration. The general ground color of the larvae of $L$. decemlineata is wine red, that of L. oblongata and L. multitaeniata chrome yellow. L.decemlineata and L. multitaeniata have two rows of spots along the side in the larvae, while $L$. oblongata has one. L. oblongata, as shown in Fig. 6o, is long and oval in outline; L. decemlineata, as shown in Fig. 69, is more rounded; and L. multitaeniata is robust in type. There are also color differences between the species, which need not concern us here. Three experiments will serve to illustrate the purpose of this paper.

In 1905 , twenty $L$. decemlineata, from a pedigreed culture, from Chicago, twenty L. oblongata, from a pedigreed culture at Cuernavaca, and twenty L. multitaeniata, derived from an isolated standard locality in the valley of Mexico south of Guadalupe, were placed on an isolated island in the Balsas River. This island was fairly well covered with a growth of Solanum rostratum, or a closely related form, upon which all three species would feed. As far as could be discovered, the island was devoid of any individuals of $L$. oblongata, which occur very sparingly in that general region, and the neighboring banks of the river and the islands were all searched, but they afforded no trace of L. oblongata. These introduced beetles were allowed to breed and gave the first hybrid generation in August, I905. In this generation only the adults were seen and of the adults we could recognize definitely five forms: (A) Those which on 
inspection appeared to be wholly L. decemlineala; (B) those which appeared to be wholly L. oblongata; and (C) those which appeared to be wholly L. multitaeniata. There were individuals which were manifestly intermediate hybrids, in
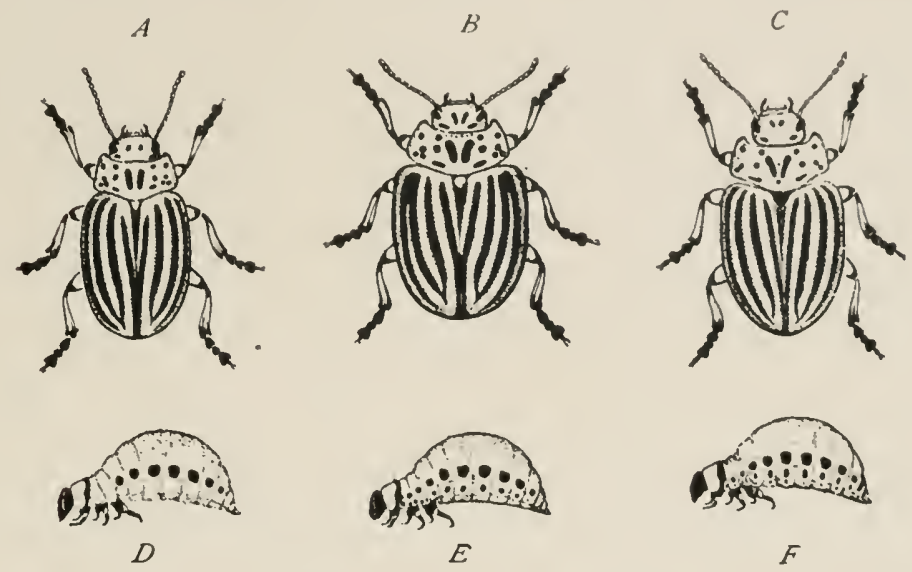

FIG. 69.-Arranged to show some of the essential differences between the species: L. oblongata, L. mullitaeniata, and L. decemlincala. (1) Showing the form and characteristic markings of the adult of $L$. oblongala. (B) Adult of $L$. mullilaeniala, showing the more robust form and somewhat different type of general color pattern sharply distinguishing it from both of the other species. The elytral ground color is often dark ochre, sometimes eren reddish. (C) The type of $L$. decemlincala used in these experiments, somewhat intermediate between the two other species in body form, and to a certain extent in markings. (D) Showing the side view of a full-grown larva, with its color pattern. The ground color is yellow and that of the adult somewhat variable. (E) Adult larva of $L$. mullilacniala, with the characteristic color pattern. Ground color is yellow as in $L$. oblongata, but darker. (F) Shows the characteristic color pattern of $L$. decemlineata; the ground color of the larvae is wine red.

form, punctation, and coloration, between L. decemlineata and $L$. oblongala (D); and between $L$. decemlineata and $L$. multilacniata (E). Of these five forms a census was made with the following results:

$\begin{array}{ccccc}\text { A } & \text { B } & \text { C } & \text { D } & \text { E } \\ 327 & 37 \text { I } & \text { I }+2 & \text { I }+430 & 2+6\end{array}$


All the individuals were allowed to remain in the colony, and interbred freely in August, giving early in September a second generation, of which the following census was made:

$\begin{array}{ccccc}\text { A } & \text { B } & \text { C } & \text { D } & \text { E } \\ 46 & \text { IOI } & 90 & \text { I }, 292 & 210\end{array}$

These hibernated during the winter of $1905^{-6}$, and were not seen again until September, I906, in the fourth hybrid generation of the culture. At this time the dominant form was manifestly a combination between L. decemlineata, L. oblongata, and L. multitaeniata, with the oblongatadecemlineata attributes in excess of those of $L$. multitaeniata (a combination between classes $\mathrm{D}$ and $\mathrm{E}$ of $\mathrm{F}_{\mathrm{r}}$ and $\mathrm{F}_{2}$ ):

$\begin{array}{cccccc}\text { A } & \text { B } & \text { C } & \text { D } & & \text { E } \\ 7 & 25 & \text { I } 2 & & 2,210 & \end{array}$

The huge preponderance of this complex type, which was neither one nor the other of the three species, suggests at once, of course, that the results could not be due to any selective process, because the type was not one of the original types but a hybrid complex.

The wintering conditions of $1906-7$ were especially rigorous, at least as judged by the number of beetles that I found in that location in 1906-7, when the following census was made:

$\begin{array}{lllllll}\text { A } & \text { B } & \text { C } & \text { D } & & \text { E } \\ 0 & 0 & + & & 422 & \end{array}$

This shows that during the winter practically only the hybrid combination was able to survive. These reproduced and gave a progeny in July, 1907. An inspection was made early in August, when I found only the dominant type present in the fifth hybrid generation.

$\begin{array}{llllll}\text { A } & \text { B } & \text { C } & \text { D } & & \text { E } \\ 0 & 0 & 0 & & \text { I, } 877 & \end{array}$

The culture was not seen again until the spring of I908, when a considerable number of the dominant form of the 
sixth hybrid generation was found emerging. These were taken to Chicago and subjected to analytical experiments and were found to breed true, both in group and in pedigreed cultures, with this exception, that in both the pedigreed cultures there occurred from time to time sporadic variants often standing a considerable distance apart from the rest of the population, which, when inbred, either with sports like themselves, or back to the parent type, gave behaviors which in every way are comparable to the behavior observed in many of the forms which are supposed to have arisen by a mutative process. These strains were kept through the years I9oS and I909, and gave results which strongly suggest that the interpretation of a mutative period as described by DeVries in O. Lamarckiana, may well be the variability which follows complex processes of hybridization.

In I 906 operations were begun at Orizaba, and in May the same three species from the same original stocks were mated. Conditions at Orizaba are decidedly different from those in the Balsas Valley. The city is 2,000 ft. higher in altitude and the climate is very different. In the Balsas Valley during the summer the days are bright and hot, with even showers. At Orizaba, in the location chosen at the foot of the Sierra Escamela, it is never above $90^{\circ}$ even on the hottest days, and the nights are always cool, owing to the downward draught of cool air from the mountains which flows over the valley at night. The relative humidity is high at all times, and the precipitation during the season was 74 inches.

Under these conditions the crosses which were made thrived as far as certain members were concerned: the L. multitaeniata individuals were decidedly reduced by the conditions under which they were living and the L. oblongata. individuals were hampered considerably, but to a lesser 
degree. Crossing was observed, however, among the component species in all directions, and progeny emerged in July, showing a combination to have been formed between $L$. oblongata and $L$. decemlineata, with the $L$. multitaeniata type and attributes wanting. The population, when examined, showed individuals which were apparently dominated by $L$. decemlineata (A) to the exclusion (as far as visible) of all others; individuals which were very clearly intermediate between $L$. decemlineata and $L$. oblongata (B); and individuals which were more or less intermediate between L. decemlineata and L. multitaeniata (C). Of these the intermediate between $L$. decemlineata and $L$. oblongata existed in by far the greatest numbers, as shown by the following proportion:

$\begin{array}{ccc}\text { A } & \text { B } & \text { C } \\ \text { I3 I } & 397 & 92\end{array}$

Inasmuch as this experiment was conducted in a large cage and not in the open, it was manifestly impossible to utilize all the individuals which emerged, so a reduction was made for the matings for $\mathrm{F}_{2}$, excepting that any extreme or rare types were given every advantage over the more common types. The following materials were selected at random from the different groups as parents of the second generation:

$\begin{array}{ccc}\text { A } & \text { B } & \text { C } \\ 35 & 3^{f} & 33 \\ 3 q & 3 q & 3 q\end{array}$

These inbred rapidly during July and at the end of August gave a second generation which was uniformly an intermediate between $L$. decemlineata and $L$. oblongata.

$\begin{array}{ccc}\text { A } & \text { B } & \text { C } \\ \circ & 589 & \circ\end{array}$


This was especially true of the adult characters. The larval characters, however, were also variable and appeared to be less blended into a homogeneous group.

The culture hibernated from early September, Igo6, to June, 1907. During this period a very great mortality occurred, which was duc very largely, I think, to the fact that the culture would probably have reproduced a third time in I9o6 if it had been supplied with food and proper conditions.

These individuals in 1907 reproduced and gave a pretty uniform progeny of the blended type between $L$. decemlineata and $L$. oblongata, Generation III :

$\begin{array}{lcc}\text { A } & \text { B } & \text { C } \\ 2 & 476 & 0\end{array}$

A fourth generation was obtained in late August and early September of the same year, which possessed the same attributes as the third generation. In nature, this culture was not carried beyond that stage, but material from the culture was brought to Chicago and carried through the winters of 1907 and I908, and the summer of I908 and part of 1909. It was subjected to various analytical experiments, all of which tended to show that the type was a relatively stable one. Individual pairs, when inbred, gave a very definite pure line culture and groups mated at random gave the same result; but, as in the colony in the Balsas River, there appeared sporadic individuals, widely separated from the parent stock, which, when inbred, behaved in every way like DeVries' mutants.

A culture of the same material was placed at the Desert Botanical Laboratory of the Carnegie Institution in the desert of southern Arizona at Tucson, near the foot of Tumamoc Hill. In this experiment two males and two 
females of L. decemlineata, from the typical stock at Chicago, two males and two females of $L$. oblongata, and two males and two females of L. multitaeniata were mated in the early part of June. This culture was çonfined in a cage $6 \mathrm{ft}$. square on the ground and $3 \mathrm{ft}$. high, covered with wire eighteen meshes to the inch, thus eliminating all selection by insectivorous enemies. $S$. rostratum was supplied as food in sufficient quantity. During June and July these reproduced abundantly and gave a large progeny which emerged late in July and early in August. In this first hybrid generation at Tucson there was, as in the other cultures, a blending of the materials introduced into the experiment, but in this culture $L$. decemlineata was the dominant member of the cross, although not completely. In the larvae six types were observed:

I. Those which on inspection appeared to be L. decemlineata.

2. Those which were L. oblongata.

3. Those which were L. multitaeniata.

4. Those which were intermediate between $L$. decemlineata and L. multitaeniata.

5. Those which were intermediate between $L$. decemlineata and $L$. oblongata.

6. Those intermediate between $L$. oblongata and $L$. multitaeniata.

It was, of course, impossible to tell on inspection what the constitution of each of these types was. Five classes of adults were recognized:

A) Those which were clearly either pure, or dominants of the L. oblongata type.

B) Those which were clearly intermediate hybrids between $L$. decemlineata and $L$. oblongata. 
C) An L. decemlincata type in which L. decemlincula was in the main dominant, but which exhibited a variable range of variability.

D) Intermediate hybrids between L. decemlineata and L. multitacniata.

E) Forms which were either L. multitucniata pure, or heterozygotes, in which L. multitacniata was completely dominant.

Out of $x, 8_{57}$ adults seriated, the following census was made:

$\begin{array}{ccccc}\text { A } & \text { B } & \text { C } & \text { D } & \text { E } \\ 47 & 29 & \text { I }, 3 \text { I I } & 26 \text { I } & \text { IO3 }\end{array}$

This census shows that while $L$. decemlincata is either the dominant or prepotent member of the combination, it did not come out of the mixture entirely without contamination.

This experiment was continued in a cage exactly like the first, and the following materials were taken at random from the first generation as the parents of Generation II:

$\begin{array}{ccccc}\mathrm{A} & \mathrm{B} & \mathrm{C} & \mathrm{D} & \mathrm{E} \\ 25 & 25 & 6 & 3 \% & 35 \\ 29 & 29 & 6 q & 39 & 3 \%\end{array}$

This material immediately began breeding and gave during the month of August a large progeny which emerged early in September, and immediately went into hibernation. When seriated, this material gave the following results:

$\begin{array}{ccccc}\text { A } & \text { B } & \text { C } & \text { D } & \text { E } \\ 0 & 29 & 247 & 42 & \text { O }\end{array}$

These passed the winter of $1908-9$ in the ground and emerged in June, I909. All that emerged were allowed to reproduce in the cage and were supplied with food as fast as it was consumed. These gave a very large progeny which appeared to be uniformly of the dominant types of the first and second generations. Seriation of the material 
obtained from Generation III at the end of August, I909, gave the following results:

$\begin{array}{ccccc}\text { A } & \text { B } & \text { C } & \text { D } & \text { E } \\ \circ & 5 & 362 & 8 & \text { O }\end{array}$

I then mated at random for the parents of Generation $\mathrm{IV}$, one male of $\mathrm{B}$, the only one that could be found alive, three males and three females of $\mathrm{C}$, two males and two females of $\mathrm{D}$, and none of $\mathrm{E}$, they being absent. This material bred at once and gave in the fourth generation a considerable progeny, which were all of the dominant type.

Material from Generation IV, brought to Chicago in August, I909, placed in hibernation under experimental conditions, and brought out to breed in the middle of the winter, has shown that the dominant type is a fixed type, and that it breeds true and does not split in subsequent generations. The only splitting is that which occurs in rare individuals in from 2 to 3 per cent of the progeny, which stand apart from the general population as sports. These cases are practically the reappearance of one or the other of the component characters or combinations thereof that went into the cross, and they do not represent in this experiment anything in the way of characters new to the genus or family as DeVries states to be true of his mutants rather, they are simply the characters obtained from the different parents from which this complex has been built up.

The same combination of material was made in Chicago in Igo8, and was run through essentially the same procedure as that of the Tucson experiment, with this difference in the result, that at Chicago $L$. decemlineata completely dominated the culture to the total exclusion, as far as analysis has been able to discover, of the presence of the other parents. 
These experiments in synthesis represent what might happen in a state of nature when species which can hybridize migrate from one place to another and intercross. No one realizes better than I the complexity of experiments of this kind, the difficulties involved in the analysis of the results, and the caution that should be exercised in making statements from them. It seems certain from these experiments, as far as they have been carried out, and they are by no means complete, that we may definitely conclude that when like materials are combined under different natural environments, differences in the products, depending upon the conditions under which the combination takes place, result. It is certain that the type which came out of the culture in the Balsas Valley was quite different from that which resulted from the cultures at Orizaba, and these are different from the dominant type which arose at Tucson.

One point of very considerable interest is the behavior of these dominant types in exactly the way in which Delries' Oenothera Lamarckiana behaves, giving in each generation, a greater or less number of rather divergent individuals, which, when inbred, are found to be stable germinal variations.

Bateson in 1902 suggested that the mutations observed by DeVries in Oenothera Lamarckiana are in reality due to some sort of hybridization behavior. I am of the opinion that Bateson's suspicion is probably justified, at least in some instances. I have no experience with plants, and especially none with $O$. Lamarckiana, but my experience with these synthetic experiments has suggested that the type of behavior which DeVries has discovered, and upon which he has built an all-inclusive theory of erolution, is in reality nothing more than the reappearance 
from time to time of attributes brought into the strain by hybridization, and which reappear in every generation, or in frequent generations, by some process akin to Mendelian segregation.

It seems unreasonable to advance, as has DeVries, the idea of a premutation period, with a gradual development of invisible pangenes, and then a final bursting of these pangenes into a full-fledged mutation period, followed by a gradual dying away of the mutation period which leaves a species in a condition in which it does not produce these sports. Rather, the explanation which Bateson suggested, and which I have shown to be capable of creation in these synthetic experiments, is far more plausible and more likely to be the real explanation of the type of behavior found.

This raises a very large question-one that has been raised many times-as to whether natural species may not be hybridization complexes rather than pure line cultures isolated by some sort of selection, as has been presupposed since the time of Darwin. I have found that in nature, crossing, especially between these chrysomelid beetles, is by no means uncommon, and very frequently results in adult progeny in nature, some of which have been described as species. These natural cases of hybridization have been observed in the last half-dozen years along the edge of the Mexican plateau. Some other species of chrysomelids. from the same general region, especially some species of Labidomera, have a variability strongly suggestive of a similar origin. I have found that Labidomera suturella. Cherr., of which many sharply marked variations have been described, gives a variability in pedigreed cultures that is strongly suggestive of the species having arisen 
through a process of hybridization. On the high rolcanic plateau of Toluca there is another type rather closely allied to L. multitaeniata, which is also suggestive of having arisen, or of being in the process of arising, through hybridization.

These conditions in nature are of course difficult or impossible to check and verify, because the past is absolutely unknown, and little or no indication of what it has been can be obtained from any source. The materials in museums and the records by systematists are utterly useless for this purpose. Apparently the only way of attacking this problem is the one which I have adopted of placing colonies in isolated locations, or in cages, there to carry out the process of interbreeding and forming of hybrid combinations as they would occur in nature.

In the last few years at Tucson a series of experiments has given an exact duplication of the "mutation behavior," and further, it is clearly called into operation by conditions external to the organism. At first a race was synthetized and was and is still constant, but when placed under optimum conditions of growth and development at Tucson it has given fourteen distinct types. Some of these had in previous experiments been tested out and are known to breed true, and others are still to be tested.

With plants, Gates and Davis are endeavoring to produce synthetically $O$. Lamarckiana from a hybridization of $O$. grandiflora and $O$. biennis, and while as yet O. Lamarckiana has not been produced, Davis has obtained a type which he considers to be very close thereto. It is perhaps not too much to expect that in the near future O. Lamarckiana will be experimentally synthetized.

If it proves to be generally true that "mutation beharior" is a sequence of synthetic composition, it does not in 
any way detract from the value of DeVries' observations, nor of the rôle which types thus arisen may play in evolution. It is true that the hypothetical portion of DeVries' theory as regards a permutation period and so on, is in part true, in part not. There is a period of synthesis, ending in a uniform stem race that may endure for a long time, and subsequently this throws off from itself gametes unlike, in that there are new combinations of old characters, reappearance of long latent characters, and not infrequently new characters.

There is, however, this essential difference between the conception of DeVries and the one that I have to offer: namely, DeVries regards species as pure in the old sense and arising by dichotomy, while I am convinced that progress will show that synthetic combinations are largely responsible for the stem forms of generic groups, and that from these there have arisen related species or types in greater or less profusion. As far as experience goes, this production of new types is in the main, if not entirely, a product of the action of external forces upon the gametic constitution, although when once started in such a strain it seems not to cease for some time though the inciting cause is removed. In Fig. 7o I have tried to show in diagrammatic fashion the essential differences of the two conceptions.

B. The Experimental Production of Germinal Variations by the Direct Action of Different Forces

I. FORCES EXTERNAL TO THE ORGANISM

Incident solar radiation in its various manifestations, the water relations of organisms, density and composition of the medium, and the nature of the food stream are the common groups of forces that are apt to be modified in 


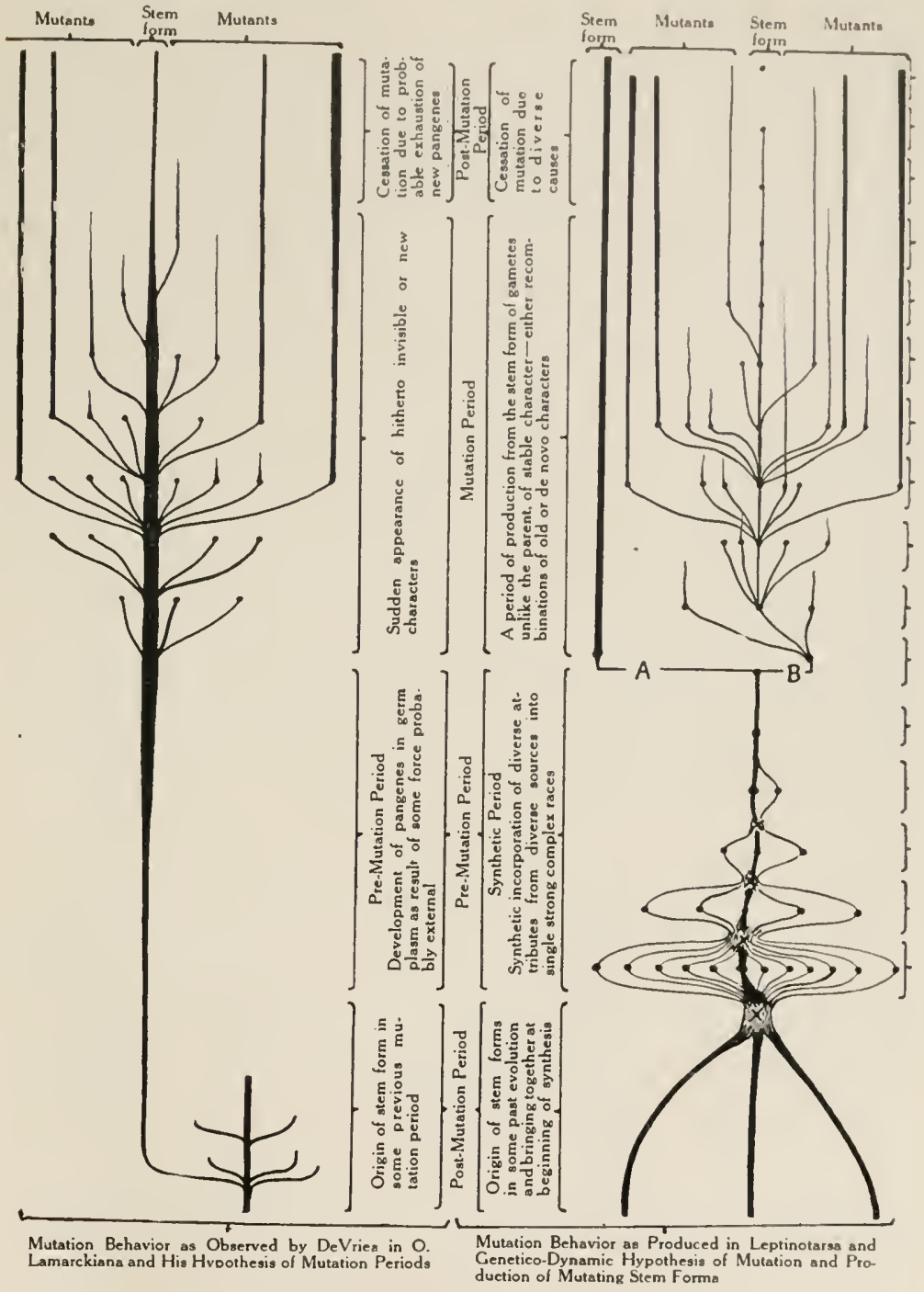

Fig. 70.-Diagram to show the differences between DeVries' theory of the origin of mutations, and the hypothesis of Mutation as a result of hybrid synthesis of the mutating stem form. 
presence, character, or intensity, in relation to the organisms, and to these forces and relations are attributed a variable value in the production of germinal variations. All kinds of extreme demands are made upon external forces, from the conception of an organism as a plastic material which is pressed into shape, and given its characters by the stress of environment, to the opposite assertion that environment acts, if at all, as a minor factor in eliminating the unfit. From logic and argument no truth may be expected, and the only hope of progress in the quest for truth in this problem lies in the domain of exact genetic research.

In this chapter-which is a summary of recent advances and not a historical résumé of the whole subject - the earlier work is not discussed, because it has been so often summarized that good discussions of it are available in many publications. All of the older work, however, is seriously defective when considered from the viewpoint of presentday genetic investigations.

\section{IN PLANTS}

In the bacteria and yeasts the refined and accurate methods of investigation now used, and the fuller recognition of the genetic requirements have made possible studies which have given much valuable information. The work of Pringsheim, Winogradsky, Hansen, Barber, Beijerinck, Buchanan, and others stands as examples of what may be accomplished in the study of this problem in these simple organisms. In all of the observations thus far made upon these organisms response to incident forces or changed conditions is immediate, and departures, often of considerable magnitude in form and function, occur; but in most instances difficulty in fixing these modified characters has been encountered. 
The well-organized experiments of Buchanan with Sireptococcus lacticus are quite characteristic of the general results obtained in the investigation of this problem in unicellular plants. His conclusion that fluctuations cannot be fixed in bacteria, whether normal or induced, is in accord with most of the experiments of bacteriologists; nevertheless, this result is opposed by many records, believed to be accurate, of sudden permanent departures in form and function, especially in the yeasts.

After a comprehensive survey of these studies upon bacteria, Pringsheim concludes that while changes in yeasts and bacteria have often resulted from the unusual action of culture media, toxic solutions, temperature, etc., the modifications of form and activity are diverse, and are either permanent or transient. Especially difficult, however, if not impossible in these organisms, is the attempt to separate somatic and germinal effects, and much reasonable doubt exists as to its possibility. The important contribution from this work with bacteria and yeasts is the precise demonstration that the departures are readily produced, and are a direct result of the incident external forces used, even in the simplest known organisms.

Other low plants, such as algae and fungi, have often been subjected to exciting agencies, and while changes have resulted, these, like the modifications in bacteria, are usually transient and not permanent. It would seem that these simple forms ought to provide good material for the study of this problem, although it is possible that the low differentiation between soma and germ may introduce experimental difficulties yet to be overcome.

In higher plants the observations of Zedebauer with Capsella are of interest, and introduce observations which 
might be duplicated and extended by proper experimentation with other plants and lead to new and important information. A biotype of Capsella, bursa-pastoris, much like taraxicafolium, lives on the low plains along the coasts of Asia Minor. It has broad leaves, white flowers, and grows to $30-40 \mathrm{~cm}$. high. On the inland plateau at altitudes of $2,000-2,500$ meters grows another form with a stem $2-5 \mathrm{~cm}$. high, reddish flowers, xerophilous leaves, and an elongated root system.

From the lowlands roads lead to the plateau, and the conditions of distribution are such as to suggest that man has been influential in disseminating this form from the lowlands to the highlands, where it has taken on the modified form. Some force to this interpretation of the distribution is given by the fact that seeds from the plains, when taken to the plateau, at once assume the somatic characters of the plateau type. On the other hand, plateau seeds, planted and grown at Vienna, while they have the xerophilous character of the leaves, retain the upland characters in flowers, and, to a large extent, in height, root system, and general habit. It is futile to attempt to draw from observations in nature such as this conclusions as to the past history or actual happenings, but such instances clearly indicate the sort of experimentation that might profitably be attempted and the type of results that might be expected.

Much more concrete and accurate are the findings of Klebs upon Sempervivum, in which inflorescences were found to be capable of replacement by a single flower, and many other changes were induced as the result of external, mainly climatic, forces. More important than the fact of the departure is the fact that some changes persisted 
through three or four generations in properly guarded cultures.

MacDougal has attacked this problem from a strictly experimental standpoint in the effort to discover a cause, and to arrive at an understanding, of the phenomena of "mutation" as described by DeVries in Oenothera. The method which he has used is to inject solutions of various kinds into the ovaries immediately before fertilization. Later, Gager, by subjecting seeds to the action of radium bromide, has shown that in plants physical factors incident upon the germinal materials can and do produce germinal changes that are permanent and persist in undiminished vigor in subsequent generations.

MacDougal's experiments, wherein zinc salts, cane sugar, etc., were injected into the ovules of plants, show that permanent changes resulted. MacDougal's method and the reasons therefor are as follows:

Having carried on pedigree cultures with a large number of species for several years and having encountered some which did and others which did not give rise to aberrant individuals, attention was directed to the possibility of inducing changes in the hereditary elements in such a manner that the qualities transmitted would be altered or destroyed. A theoretical consideration of the subject seemed to indicate that the changes constituting the essential operation of mutation ensued in a stage previous to the reduction divisions in the embryo sac, or the pollen mother cells. It was planned therefore to subject these structures to the action of chemical agents, not ordinarily encountered by the elements in question, at a time before fertilization occurred. The tests were planned to include the use of a solution of high osmotic value, and mineral compounds, some of which are toxic in concentrated solutions and stimulating in the proportions used. The probability of success would be heightened with the number of orules contained in any ovary operated upon, and therefore the common evening primrose, Oenothera biennis, Raimannia odorata, a relative of it, and a 
member of the same family, Begonia, Cleome, Abutilon, Sphaeralcea, and Mentzelia, and others were experimented upon. Without recourse to the detail of the work it may be stated that the use of radium preparations, sugar solutions (Io per cent), and solutions of calcium nitrate, of distilled water, with capsules of Raimannia odorata, and zinc sulphate in a stronger solution used with Oenothera biennis (Fig. 7IA) was followed by very striking results. In the first-named plant, there appeared in the progeny obtained from a few capsules of one individual several individuals which were seen to differ notably from the type with the appearance of the cotyledons, and, as development proceeded, it was evident that a mutant had appeared following the injections and nowhere else, which thus had some direct relation to the operation. The characters of the newly arisen form were so strikingly aberrant as to need no skill in detection (Fig. 7 I $B$ ). 'The parent was villoushairy, the mutant entirely and absolutely glabrous, the leaves of the parent have an excessive linear growth of the marginal portions of the leaf blades and hence become fluted; the excess of growth in the mutant lies along the midrib and the margins become revolute. The leaves are widely different in width, those of the mutant being much narrower. The parental type is of a marked biennial habit and near the close of the season the internodes formed are extremely short, which has the result of forming a dense rosette; the mutant forms no rosette by reason of the fact that the stem does not cease, or diminish its rate of elongation and hence presents an elongated leafy stem, which continues to enlarge as if perennial. The first generation of the derivative came to bloom; the flowers of the mutant were closely guarded and as soon as seeds were obtained they were planted to obtain a second generation. A few plants were obtained, which in every particular conformed to the new type and exhibited no return to the parental type.

MacDougal's investigations, wherein were produced modifications that have remained stable through four or more generations, in Raimannia, Cereus, Penstemon, and others, show fully that the method employed gives definite changes in germinal constitution. 

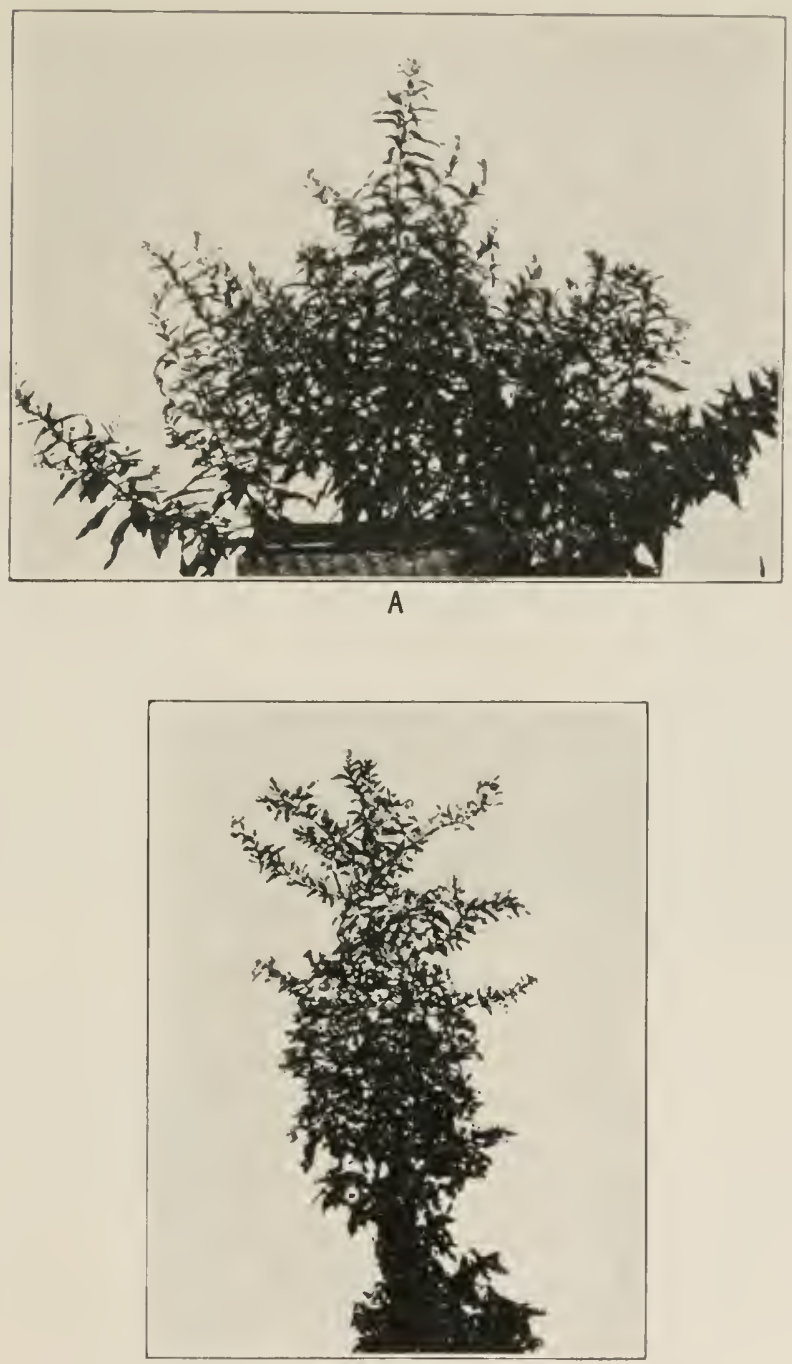

B

FIG. 71.-Two plants of Onagra biennis, showing the effect of injections of zinc sulphate into the ovule. $A$, normal. $B$, the modified plant, which arose from seeds that had been modified by the zinc sulphate. (From MacI)ougal.) 
In Gager's experiments the action of radium rays and emanations upon plants are definite, and in some instances permanent modifications resulted (Fig. 72). In these experiments modifications by physical and chemical agents were produced which are not necessarily pathological, and some of them continued to breed true in subsequent generations.

Gager found that the action of radium rays upon pollen cells was to produce distortion of the karyokinetic figure to the extent that chromosomes were left entirely out of the spindle and were lost to that particular germ cell. What happens in any particular variant whose modifications are inheritable has not been determined, but the suggestion is at least plausible that the radium emanations in some way produce a new, or bring about a rearrangement of the physiological complex which exists in the germ cell. Conceivably it may be due to the displacement of an individual chromosome, although this suggestion would need verification before it could be adopted.

The modifications induced by the injections of salts in MacDougal's experiments are not easy to understand. The cells of the ovule are relatively impervious, and there is a relatively small amount of dispersion from the seat of the wound. The results obtained, however, are not due to the effects of wounding, as shown by the fact that ovules wounded in the same manner do not produce modifications unless the salts are present; likewise, ovules stung by insects do not, as far as known, produce these results, and it is only in ovules into which chemical salts have been injected that modifications are effected. The conclusion seems unavoidable that the salts injected produced the observed results by modifying in some way the constitution of the 


$$
\frac{2 \pi}{1}
$$


germinal substance. MacDougal's general conclusion, as to the manner of producing this result, is that the action of the injected chemicals is to accelerate or retard processes, especially those of a katalytic nature within the germ cells, or possibly, actual chemical changes in germinal substance may be affected.

\section{IN ANIMALS}

In animals the most satisfactory results have been obtained with higher types, while among the lower types, Protozoa, for example, changes by incident forces, though capable of production, behave in much the same manner as do changes induced in bacteria and yeasts. In some instances, variations persist for many successive fissions, but they usually occur in only one of the individuals of each pair, as in Jennings' Paramoecium with a spine. In these instances there is little or no spread of the change in the population through reproduction and thus far no long-continued strains have been developed through these agencies. The condition of protozoans in the non-differentiation of soma and germ, as in bacteria, is a complication not easily overcome in experiment, and it may well be, as has been often suggested, that the entire organism is the "germ plasm."

Attempts to produce germinal changes by the direct action upon the germ of external agents have not been made by many workers. Many, it is true, have subjected organisms to changed conditions and obtained modifications, aberrations, but relatively few tests have been made to determine the inheritability of these changes.

The recent experiments of Woltereck with Daphnia and Sumner with mice are good examples of a common type of investigation, Woltereck carried strains of Daphnia in cultures in which over-feeding was practiced for about 
two years. At the end of this period it was found that the head form had changed and that when the modified form was put back into the original conditions the changed form was retained. Unquestionably, in Woltereck's cultures a head form, different from the one with which the culture started, was present at the end of two years, and this did not revert on return to normal conditions; but the failure to carry adequately controlled parallel normal lines does not permit a decision as to whether the change is a real one, or due to the progressive selection of a biotype present but obscured in the original population. Nor do the experiments permit of a decision as to whether the effects observed were due to direct germinal modifications or to somatic transmission. It is shown that a permanent change of the race resulted, and nothing more. Parallel cultures and much more careful experimentation would be necessary in the effort to answer the more important points.

Similar in method and in results are the experiments and conclusions obtained by Kammerer on certain amphibians and Lacertilia. For example, Salamandra maculosa is ovaviparous in the lowlands, but its highland variety, $S$. atra, is viviparous, and the larvae are large when born and have long gills. It was found that lowland forms of S. maculosa kept without water at low temperatures showed reproductive habits and young much like those in $S$. atra in the alpine regions. In the lizards, temperature was found to be productive of color changes, giving dimorphism in the males of one species and in the females of another. These changes are alternative in crosses.

In both experiments the results have not been carried far enough to test the inheritance thoroughly, and moreover, the conditions of experiment do not permit of any analysis 
of the process at the bottom of the observed changes. In both, there are changes following altered conditions, and there have resulted changes in the organism which are known to occur in many instances, only in these experiments some effort was made to test the permanency of the variation and its behavior in subsequent crosses. In both, the change seems to be a germinal one, as is indicated by its behavior in inheritance. Whether the change is a direct germinal or an indirect one, due to somatic influence or transmission, the experiments cannot decide.

Precisely similar are Sumner's experiments in subjecting mice to high and low temperatures, where at the end differences were found which were attributed to the effect of the different conditions. Differences there were at the end, but in mammals so variable as mice carefully pedigreed strains free from biotypes should have been used, and adequate parallel controls should have been maintained. In that controls of critical character were lacking and the possibility of biotypes was not eliminated from the stock used, the results obtained are easily attributed to gradual selection of biotypes or of actuation of latent characters, as well as to the effect of changed temperatures. As for the question of somatic influence or direct germinal effect, the experiments are not conducted so as to give proper evidence thereon and are capable of any interpretation. The experiments show, however, that changed conditions changed the stock, which change may have resulted from any of the methods suggested, and the change is apparently permanent although the series was too short to answer this question adequately.

In insects, I have obtained modifications in various ways, some of which will be described in a later portion 
of the chapter. Morgan, in Drosophila ampelophila Low, found that sex-limited variations of pink eye, etc., have apparently followed the treatment of cultures of this animal to the action of radium bromide. Loeb, however, using the same organism, found that in both experiment and control the variation described by Morgan appeared, and of course concluded that the tendency to produce this variation was already present in the race of flies used.

Lutz had for several years used the same strain of Drosophila for experiment, and found much variation in wing venation. He also subjected the strain at times to different experimental conditions, and this may be responsible for the appearance of the variation found by Morgan following the use of radium, and which appeared in experiment and control in Loeb's experiments.

\section{In Chrysomelid Beetles}

In the modification of the germinal constitution by experimental means it must be known as certainly as is possible whether there are in the germ potential capacities, i.e., latent characters, which ordinarily are not visible in the materials used, but which may be called into visibility, periodically or rarely, by unusual conditions. Unless possibilities of this kind are eliminated, it becomes difficult in experiment to decide whether observed results are the product of latent conditions, or of the experiment as de noio variations. Moreover, experiments to show the effect of incident conditions upon the germinal material must, beyond any question, show that the effect is primarily upon the germ and not first upon the soma of the parent, and secondarily, by transmission, to the germ. If, in experiment, the soma of the parent and of the resulting 
progeny be modified in the same manner, there are two possible explanations. The incident conditions may modify both soma and germ independently, and they would be similarly modified because both soma and germ represent one and the same group of potentialities; or the observed results can be explained by assuming that incident conditions first modify the soma, and secondarily, through transmission, the modification is incorporated into the germ cell; and thus be interpreted as upholding the neo-Lamarckian idea of the inheritance of acquired soma variations. It follows, therefore, that the germ cells upon which experiments are to be carried out must either be taken from the body of the parent and placed in indifferent media before being experimented upon, or they must be in organisms that can undergo no further somatic modifications. In this there could, of course, be no transmission of acquired variations, because no variations are acquired. Moreover, for our purpose any resulting change must be thoroughly tested by subsequent breeding for many generations.

When these organisms attain sexual maturity, they have attained all of the somatic modifications, save pathological growths, which it is possible for them to achieve; the ontogenetic development of variations has come to a standstill, the whole activity of the organism is directed to reproducing the species, and further development or divergence in any of its attributes or qualities is forever inhibited. Whatever changes occur, from sexual maturity onward, are pathological or senescent.

It is possible, therefore, to eliminate from these experiments the neo-Lamarckian factor, because the conditions of experiment were not applied until after the parents had 
attained full sexual maturity and complete development of all qualities and attributes.

A check upon the possible latent characters is more difficult, but in this material it is easily carried out in one of two ways: First, only pedigreed material was used in experiment. This material has always been tested by crossing within and without the species to discover, as far as possible, any characters which might be present in invisible conditions. Thus far, no attributes of this description have been discovered. Second, a check was kept upon this possible source of error in the following way: these beetles have the habit of maturing their eggs in definite rotation; that is, a batch of eggs is developed, fertilized, and laid; then a time interval elapses during which another batch of eggs is being developed, and this is repeated many times, thus giving isolated lots of eggs, each separated from the one before by a time interval of from two to sixty days, or even more, and any one of which may be subjected to experiment and the others used as controls. In many of these experiments color has served as a useful character for study and the results from the experimental modification of color may be presented first.

Experimental modification of color.-Color modifications are of two distinct kinds: changes in the pigment itself, and in the localization thereof. The first is a chemical change produced by a rearrangement of the chemical activities existing between a chromogen and an enzyme which brings the color-forming compounds into existence, while the latter is a change in the localization.

Variations in color usually are either accentuations or diminutions of existing color, and these changes are perma- 
nent, are not pathological, and the individuals possessing them are by no means weaklings, which has been shown in the case of $L$. pallida (Fig. $73 B$ ), which was produced in considerable numbers as a variation of $L$. decemlineata Say,
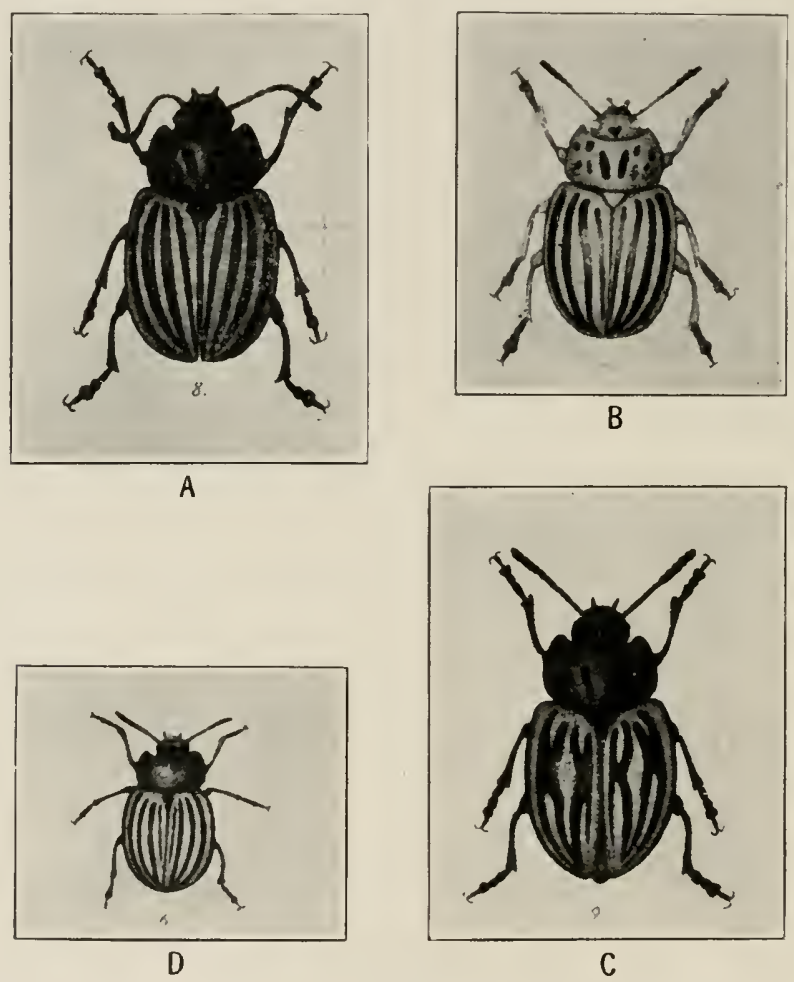

FIG. 73.- Some divergent types of beetles produced by subjecting the germ cells to external influences. A, normal decemlineata. B, the form pallida. C, tortuosa. D, defecto-punctata.

in some of my earlier cultures. This modification represents definitely a decrease in the pigmentation values of the organism, with slight changes in bodily proportions, punctation, habits, etc. It arises by subjecting the organism to rigor- 
ous conditions, especially high temperature accompanied by low relative humidity. Such variants have been found in nature, some of them are known to breed true, and others are produced in experiment.

Fig. 74 shows a photograph of a demonstration case exhibiting the results from an experiment of this kind. Here the parent pair are shown producing the two first lots of eggs which developed into normal individuals which were in following generations true to type, while the third, fourth, and fifth lots of eggs were experimented upon and gave in each modified types, pallida and minuta, as well as some normals. These different types, when inbred, came true to type in subsequent generations, as shown.

Another divergent type which arose in the same series of experiments is one in which the pigmentation is increased, that is, the amount of dark pigment in the color pattern gave the resulting individual a different appearance from that of the parent species. Both pallida and melanicum (Fig. 73.4) were true breeding germinal variations, and pallida when given a fair start showed itself, at least in certain cultures, to be capable of sustaining itself in competition with the parent species; melanicum, however, did not exhibit any such potentiality.

If a form like L. pallida were to develop in an arid area it would be recognized, as I have suggested, as a step in the process of evolution, and it would be directly attributed, if it were found in nature, to the conditions under which it was living, and its existence would be explained either by natural selection, or some other of the current hypotheses.

Other modifications which have arisen from L. decemlineata, especially in the modification of the hypodermal lipoid pigments, are distinctly not the increase or decrease 


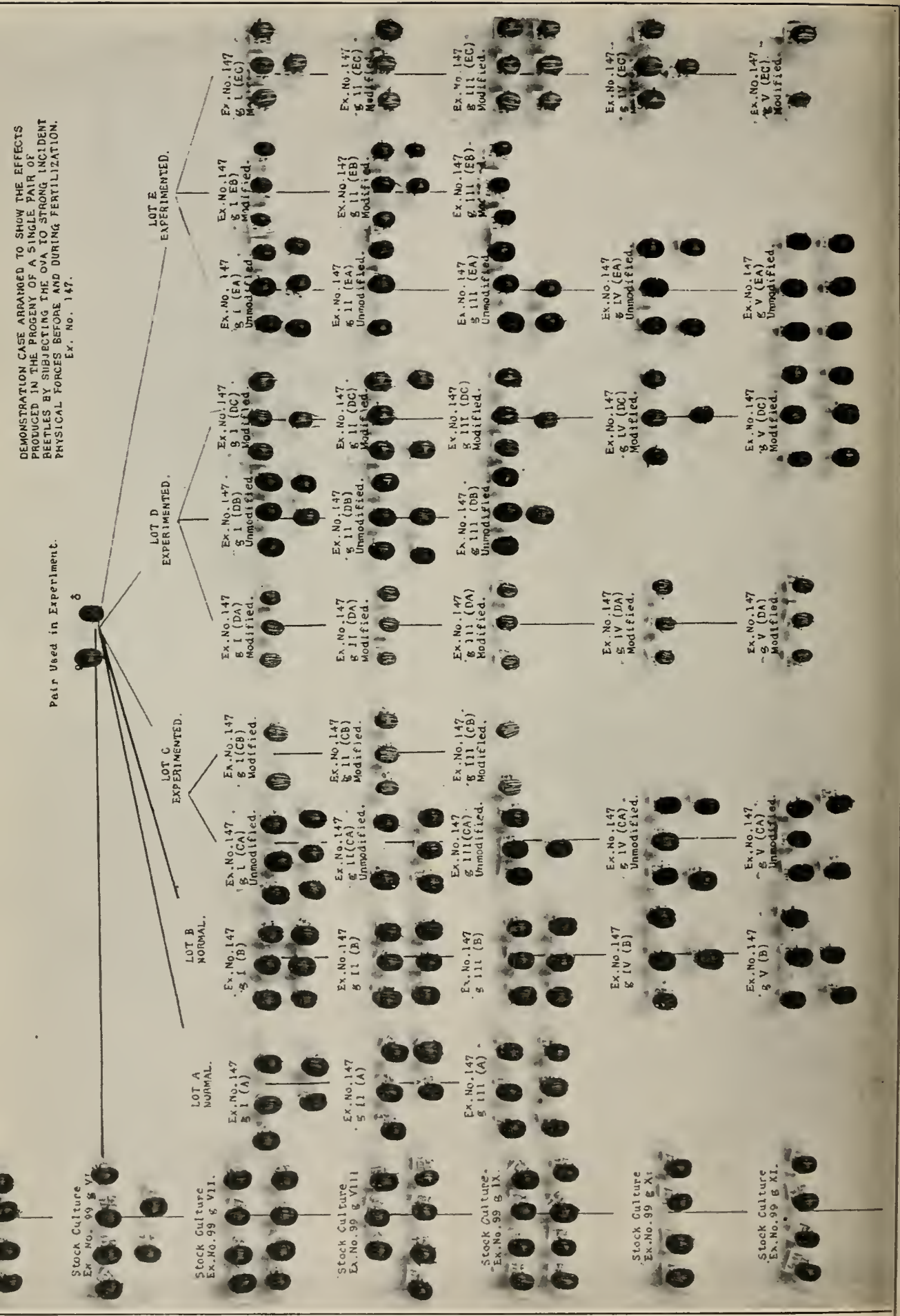


FIG. 74.-Photograph of demonstration case which has been arranged to show the effects produced upon the progeny" of a single pair of beetles by subjecting the ora to strong incident physical forces, before and during fertilization. To the left is represented the normal stock, showing no presence during the time of experiment of the modified forms. The first two lots, $A$ and $B$, were laid and matured under normal conditions and gave normal types. Lot $\mathrm{C}$, laid and matured under the conditions of the experiment, gave part modified and part unmodified forms. The same is true of Lots D and E. The modified types in this experiment were mainly pallida, clearly distinguishable in the photograph by their lighter and smaller form; they breed true in successive generations as indicated in the demonstration case. 
in the amount of pigmentation, but represent the rapid changes which are characteristic of lipochrome pigments: for example, in rubrivittata, in which the most striking character is the bright red hypodermal color. This arises suddenly as the result of experimental conditions and reproduces itself when bred back to the parent species, giving a color segregation into rubrivittata and hybrids, the rubrivittata breeding true. The behavior of rubrivittata is such as to suggest that in its main distinctive character it is recessive to the parent species.

In modifications of color we are dealing with superficial chemical processes in the organism-the development of a chromogen and the oxidation of that chromogen by one or more oxidizing enzymes to a state of stability where simpler compounds are produced which are productive of color. In no experiments in the modification of color, especially in colors such as the melanins, etc., has any modification been produced which is not an accentuation or diminution of the oxidative capacity of the organism. That is, in the case of pallida, there is a decrease in the capacity of the organism to produce either (I) the necessary oxidizing agents, or (2) the requisite amount of chromogen, or (3) the capacity of the organism to sustain the conditions necessary for the oxidative processes a sufficiently long time to enable a given amount of chromogen to be oxidized to produce a stated amount of pigment.

It has long been known that the series of color changes may proceed from white, through yellowish, yellowishbrown, reddish-brown, deeper browns, and finally to black, then to a still further stage of oxidation-white, so that with a given amount of chromogen and a given amount of oxidizing enzyme, diverse results can be obtained from 
identical materials, simply by the duration of time which the process is allowed to act. This can readily be shown in any of this material in the ontogenetic development of the individual. The oxidative process may be stopped at any stage, and the coloration of the organism then remains at the stage in which the oxidation was stopped. On the other hand, the process may be continued for an abnormally long time, producing an unusual amount of pigment substances, giving the organism a dark appearance.

We are ignorant of any mechanism in the germ cell which would bring about such inhibition of the oxidative process, supposing that the amount of chromogen and oxidizer remains the same. Of course one could, in explaining this condition, adopt the idea developed by Davenport of the existence of inhibitors, and while it is highly probable that there are such inhibitors present in the germinal mechanism, information concerning them is so fragmentary that any extended use thereof in explaining these phenomena would better be postponed.

The interpretation of the results produced is that the germinal complex has been permanently modified in some way, such that either the chromogen is not present in sufficient amount to produce the color of the parental generation, or the oxidation is deficient, but which of the two possibilities is correct cannot at the present time be decided. It is entirely probable that it might be one in some instances and in another the other factor that was deficient, both conditions bringing about identical results. It does not follow from this that there exists in the germ a definite representative of the chromogen and of the katalytic agent.

In the germ cells, as in all other cells, oxidases and substances which may serve as a chromogen are present and 
this capacity, therefore, for the production of pigmentation is not something which is conditioned by any particular thing in the cell, but it represents a capacity common to all living substances.

In what way is the constitution of the germ cell modified so that the organism shows in subsequent generations a permanent change in its coloration? It has been pointed out to me by Professor Morgan that in these experiments the behavior in the first generation is difficult of interpretation. In these experiments the male cells or the female cells, and sometimes both, have been subjected to conditions of experiment at a susceptible stage. Eggs are most susceptible immediately before and during maturation, although what connection this has to the maturation process is not known.

Morgan has raised the question, why do individuals, developed from eggs which have been subjected to conditions of experiment and fertilized with normal sperm, not give a subsequent hybrid behavior? No hybrid splitting has ever been found in any of my experiments, or in those of MacDougal or Gager. The resulting modification reproduces itself true to type, and does not give subsequent splittings suggestive of the combination of different factors or unit-characters. If there are unit-characters, it is logical to expect that in experiments of this kind the experiment would modify the unit-character in the germ plasm, and that this modified unit-character would then behave, when crossed with its normal homologue, exactly as hybrids do in other cultures. The total lack of this behavior in my experiments, and those of MacDougal, Gager, and others, might be considered good evidence that there are no such things as unitcharacters, nor in the germ cells any potentiality capable 
of individual removal or behavior. Any such deduction, however, is unwarranted and contrary to known facts, and, furthermore, these modified characters themselves show that after establishment they are alternative and capable in many instances of replacement and recombination in full conformity with established principles of heredity behavior.

How shall this behavior, which has been observed by MacDougal, Gager, myself, and others, in the production of these variations, be interpreted? MacDougal's interpretation, in the case of plants, is that the modifications induced are due to the modifiability of the enzyme action in one way or another. Gager attributes the activity to the derangement of the chromosomes by the radium emanations. In beetles, I do not know that the chromosomes are deranged by any of the processes, and we do not know that the chromosomes are the specific bearers of any particular attributes. The explanation which appeals most strongly to me in the case of eggs which have been subjected to strong incident forces is that the change should be regarded as an example of stereoisomeric change, whether in the composition of the katalyzing agent, or in the composition of the chromogen, or in some accelerator or inhibiting agent in the germ plasm. It is quite conceivable that the change may take place in the chromogen. As far as known, all chromogens are substances of wide distribution in all organisms with slightly different chemical characteristics, and it is highly probably that there is a wide range of chemical composition in these chromogens, so that a slight change in the arrangement of the molecular composition of the chromogen could be productive of the results observed. At present, however, there is little possibility of obtaining definite evidence along this line, because the present methods 
of physiological chemistry are so exceedingly gross that when by any available method either chromogen or enzyme have been removed, they have been changed to a very considerable degree in structure and relations, and possibly in capacity for pigment production. The complexity and almost futile nature of the chemical side of this problem is clearly indicated by the statement (Meischer) that in albumen molecules containing no more than forty carbon atoms there are something like a billion possible stereoisomeres. It is at once evident how utterly hopeless it is with present methods to expect exact chemical determinations of these germinal changes, and the best that the physiological chemist can be expected to do is to show the grosser outlines of the possible processes involved. To determine the exact changes within the germ cell is at present not possible.

Furthermore, the results of Reichert and Brown upon the investigation of haemoglobin crystals have shown a great array of crystalline forms and structures in this substance in allied mammalia, and it is highly probable that other substances throughout the organic world are equally divergent, equally complex, and equally specific. We must therefore keep in mind that in these color modifications there are always three possibilities - the modification of the chromogen base, the modification of the enzyme, or the modification of the capacity for carrying on the process; but present evidence, I believe, warrants a stronger belief in the efficiency of modifications in the capacity of carrying on the process more than in the modification of either the chromogen base or the katalyzer, i.e., to modified accelerators and inhibitors.

In colors due to lipoids, modifications thereof might be attributable to changes in the chromogen base, in the 
katalyzer, or, in some cases, perhaps, to the stereoisomeric relations in the molecules which are attached to the fatty base. The well-known changes which are possible in the case of lipochrome colors, changing with sharp alternativeness from red to yellow, from orange to white, or vice versa, are quite possibly due to reversibility in some enzyme within the germ cell, and this reversed action remains reversed until such time as it is again changed in its direction by incident factors. That this is apparently the correct explanation of the behavior of this particular type of pigmentation activity seems to be fully shown by many experiments.

In many of my experiments germ cells that were produced at a time when the lipochrome pigment in the parents was yellow, produced yellow progeny, but when the color had been experimentally reversed they gave white, orange, or red progeny, depending upon the direction of change in the parent. These results strongly indicate that the interpretation which I have placed upon this type of germinal variation is the correct one.

Variations in these lipoid color characters are common in plants and animals, and characters based upon these are widely used as specific differentials, and they are permanent so long as a given state of equilibrium exists. This state of equilibrium, however, can be reversed or upset, producing reversed conditions, and these reversed conditions can again, after an indefinite period, be brought back to the first condition, or some other condition, by incident forces. It is almost heresy at the present time to suggest the possibility of reversibility in evolutionary action, but the reversible nature of many characters of importance in evolution is by no means unthinkable and is susceptible of experimental 
investigation. The dogma of the irreversibility of evolution processes, which has grown out of phylogenetic and ontogenetic study, is incompatible with a physico-chemical interpretation of nature, and has no basis at present in critical experimental investigation.

These germinal modifications of color characters indicate an approach to an understanding of germinal variations in certain attributes; that is, it is understood what might happen, but in no case is it known what did happen, nor how. These variations in color concern superficial attributes in the economy of the organism; and the mere production of a color-producing substance is to a greater or less extent only an incident in the life of the organism. Colors are produced pathologically or otherwise in these organisms, by wounding, by disease, etc., at will, showing that there is throughout the organism the capacity for the production of color compound which lies at the basis of the normal coloration. The greater problem lies not in the production of color changes, but in the processes which are productive of the localization of pigments into a color pattern, and it is this attribute of pattern which differentiates organisms most certainly from inorganic substances. What is it that is productive of pattern, and in which way may the color be modified?

Experimental modification of pattern.-The pattern is an attribute by no means so easy to modify as is color, but it has been found that the pattern is less modifiable by incident forces than other parts of the organism. Of those instances in which the pattern has been modified, as in albida, tortuosa, minuta, and defecta punctata (Fig. ${ }_{73} C$ ), all proved to be germinal variations and to breed true, but the difficulty in breeding many of them and their inability to exist under 
the conditions into which they were born lead one to conclude that what is actually produced in many of these modifications are pathological germinal variations, rather than healthy germinal conditions which can be perpetuated indefinitely in nature. It should further be noted that in these modifications the changes were essentially in the amount of pigment which was produced in definite areas, especially in the color pattern of the pronotum and elytra, and while the change existed through the body as a whole, it is not thereby established that the fundamental pattern upon which these attributes are based was in any way altered. All that is certain is that there was produced a permanent modification of the capacity to produce pigment in the form of spots or stripes in definite areas.

In these modified organisms the capacity to produce pigment is by no means absent, as for example, in albida, where dark color could be subsequently produced upon any part of the body by wounds, etc., giving a considerable amount of the same dark pigment in the hypodermis and in the lower layers of the cuticula. The capacity to produce both the oxidizing agent and the chromogen is present, but the pigment is not produced in a definite location; in other words, whatever it is in the organism that determines localization is inactive, and pigment production is inhibited by some unknown inhibitor.

The production of variations in pattern by means of intense stimuli has not, in my experience, been accompanied by what I regard as conspicuous success. Variations have been produced, but these variations are often of a pathological character and could not be produced under the conditions imposed by nature. Permanent variations, however, in the pattern are obtainable by a combination of external 
forces with selective accumulation, provided the impact of the external forces is not made too intense. In other words, variations properly combined lead to more definite results in the modification of pattern than the more vigorous methods which are productive of permanent changes in color. It is therefore necessary to distinguish between the modifications of color and modifications of color pattern, because pattern may well be present as an attribute without revealing itself, and it only reveals itself when something in the organism results in the deposition of color in the proper location.

In the experimental modification of organisms, the pattern has been one of the characters least influenced. In DeVries' experiments with plants the pattern was modified relatively little, if at all, the principal changes in Oenothera being in leaf proportion, leaf arrangement, color, and similar characters, which are properties of the whole and which are known to vary with more or less readiness in all organisms. In the same way the experiments which have been carried out on insects by Dorfmeister, Weismann, Fischer, Edwards, Standfuss, and many others, show modifications in the color and little or no modification in the pattern. In some specimens the pattern is obscured by the spreading of the color from the original area into contiguous portions, but the fundamental pattern itself is not altered, as shown by the fact that in most of these experiments in the next generation the progeny revert to the pattern of the normal parental stock.

In MacDougal's experiments with plants, and in Gager's also, the pattern appears to be only slightly modified, if at all, and in all of the plants used by them the pattern was relatively simple, both petals and leaves being self-colored, 
and the variations which occurred were mainly variations which resulted from modifications of the growth processes, influencing leaf proportions, and such characters as pubescence, color, etc.

In experiments with beetles, I have found that the subjection of organisms to unusual environmental stimuli did not as a rule materially change the pattern, and never, as far as I have observed, did it in any way alter the fundamental pattern basis of the organism. It might appear on superficial inspection that the pattern existed in its original simplicity, even if it were not manifest to the eye. For instance, in defecta punctata, it is the inability of the pigment, through some cause or other, to be developed in particular areas. This inhibition of pigmentation may be due to the oxidizing of the pigment to a colorless state. On the other hand, in variations like melanicum, in which the color pattern of many of the parts is distinctly different, as far as superficial inspection is concerned, the underlying pattern is unaltered, and that which goes to make up the difference between melanicum and the parent species is simply added development and extension of the color from the original areas around which they develop. This is shown by the fact that during the ontogeny in melanicum there is an exact recapitulation of the stages through which melanicum has passed in reaching its present state. This represents an accentuation of existing characters along definite lines.

In variations of another kind there are indications that fundamental changes in the pattern occur. L. melanothora.x Stål, bears a relation to the species $L$. multitaeniata Stal either of a constantly recurring mutant which is unable to survive the conditions under which it arises, or may represent the constant reappearance of a species which 
has been absorbed by multitaeniata, but not completely incorporated-traces of it occurring in every generation with greater or less frequency. Both the melanothorax and multitaeniata develop among the progeny of the same parents, and between them there is a striking difference in the development of the pattern. Both start from essentially the same base, but melanothorax diverges with great rapidity and does not pass through the stages of the parents from which it came, as shown in Fig. 75 .

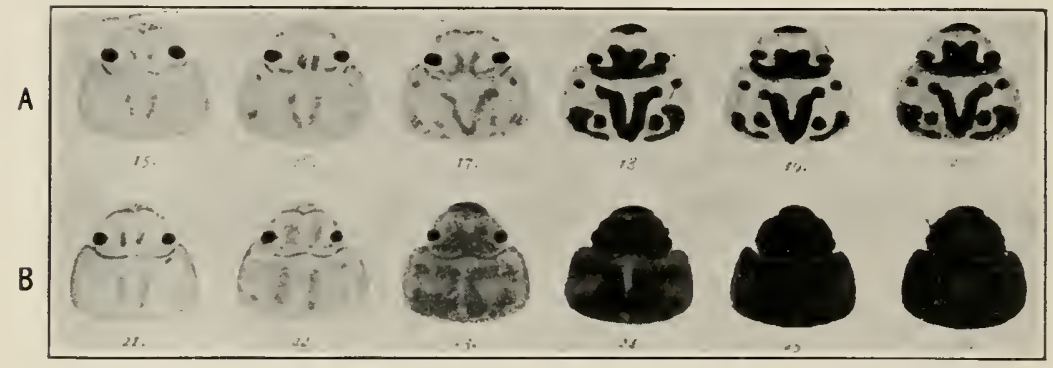

FIG. 75.-To show the sequence of stages in ontogeny in L. multitaeniata (A) and its recurrent mutant, melanothorax (B). It should be noted that the latter form has its own type of development and diverges from the parental condition, having very little in common with L. multitaeniata from which it came. This suggests that this type of development is as much a specific and independent character as is its final form.

In a pure strain of L. multitaeniata Stål, where no traces of melanothorax were observed for several generations, injections of dilute solutions of calcium nitrate caused it to give melanothorax. In these induced forms the ontogeny of the color pattern is the same as in the development of material found in nature. This behavior of melanothorax represents another type of pattern modification-a rapid divergence from the initial state common to a very wide range of species, and in no wise is it a repetition of the 
ontogenetic history of the parent from which it came. In view of the fact that the real relation which exists between L. multitaeniata and $L$. melanothorax must remain unknown, no conclusion as to the significance of this wide divergence in the development of the color pattern can be safely drawn. The relation between $L$. multitaeniata and $L$. melanothorax may be that of two hybridizing species in which L. melanothora.x is in the process of assimilation, and if this attribute is a "unit-character" it follows that in the reappearance of the recessive form which $L$. melanothorax: appears to be, it is to be expected that the recessive character would exhibit the ontogenetic series of stages characteristic of itself, and not those of the parent species out of which it came. There is, however, another possibility, namely, that the reappearance of $L$. melanothorax in each generation of L. multitaeniata may be considered a recurring mutation which diverges in its direction independently of the parental type.

This observed condition can be explained on the basis of multifarious mutations in the sense of DeVries, or on the basis of unit-characters recessive to $L$. multitaeniata, but recurring with greater or less frequency. Under conditions of nature it is not possible to determine which one of these two possibilities is the correct one.

\section{HYBRIDIZATION}

The results obtained by the horticulturist and husbandman through hybridization in achieving the modifications of plants and animals which are desired have long been known, and in plants a very considerable array of the modifications produced never get beyond the first hybrid generation and are perpetuated by cuttings, or other forms of 
asexual reproduction. In animals a considerable array of domesticated forms are definitely the product of hybridization. Poultry, for example, are derived from two or three original stocks which have been intercrossed numberless times, and from these crosses have resulted variations in the arrangement of the parental characters and combinations of characters. The same is true of domesticated pigeons, where hybridization has been carried out to a very great extent, and in dogs, cats, mice, rabbits, rats, guineapigs, swine, and in practically all domesticated organisms essentially the same condition exists. Unfortunately, in domesticated organisms the beginning of these modifications is shrouded in antiquity, and while man has reared dogs, swine, pigeons, and poultry for a long period, and while there have arisen under his hand the variations which are now seen under domestication, the manner of origin is unknown, and the best that can be done is to make plausible guesses as to what the procedure really was.

The modification of both wild and domesticated stocks through hybridization is well known to all students of hybridization, and these modifications are of two general categories: First, the production of new combinations of existing attributes, and second, the origin of de novo variations based upon the attributes of the crossed stocks. The first is by far the most common change, and the second is relatively rare in occurrence. I may illustrate the results produced in these two kinds of modifications by crossing, by examples taken from my cultures.

L. signaticollis has in the full-grown larvae one row of black spots on the dorsal side, with a deep chrome-yellow body color, and in the adult the elytra are marked with irregular rows of impressed punctations with a deposit of 
black pigment at the bottom of each. By a process of hybridization and extraction it is possible to obtain a race of signaticollis which, if found in nature, would be regarded as a distinct species; and when it occurs in experiment it behaves with the same sharp alternative distinctness of any natural species.

If a female of $L$. undecimlincata, which has the larval ground color white without any black spots on the back, and with one row of black spots surrounding the spiracles in the adult larvae and the impressed punctations very regular in pattern with the rows closely parallel, is crossed with a male of $L$. signaticollis, the $F_{x}$ generation gives two types of larvae $(y \mid s)$ and (whs), in the proportion of $\mathrm{I}: \mathrm{I}$. From the (yls) larvae will come a type intermediate between the two, and this type, when inbred, gives in the second hybrid generation four types of full-grown larvae ( $w h s)$, $(w / h S),(y l S)$, and $(y l s)$. From the (whs) larvae in the second generation are obtained three classes of adults: like the female parent, L. undecimlineata, like the male parent, L. signaticollis, and intermediate between the two.

If, now, a cross be made between these extracted $\mathrm{F}_{2} u n$ decimlineata types with the extracted $\mathrm{F}_{2}$ signaticollis type, we get in $\mathrm{F}_{3}$ mature larvae which are $(w h S)$, and these give mid-type adults in which the elytral punctations are arranged in closely parallel rows like the undecimlineata type. These, when inbred, give in $F_{4}$ larvae which are $(w h S)$ like the undecimlineata type, and these larvae give three types of adults: like the undecimlincata type, a mid-type, and like the signaticollis type.

The signaticollis type which comes out of these crosses is the modified signaticollis type, and these, when inbred, have a life cycle shorter than is normal to signaticollis. 
Its larval stages are totally different from the larval stages of signaticollis, and the general appearance of the organism is totally different. This type, derived from these variations by a series of hybrid modifications, if placed side by side with the normal species is so strikingly different that if found in nature no one would hesitate for a moment to designate it a distinct Linnean species. Not one of the characters in this form is new, and each is directly traceable to one or the other of the parents-but the form shows a new arrangement of these attributes.

In this species a germinal modification, obtained through hybridization, resulted, in which a rearrangement of attributes produced new combinations which are stable under the conditions of existence. It cannot be said in this case that anything new has been produced, only that existing characters have been rearranged and produced a combination hitherto unknown. This is the commoner type of modification through hybridization; there are, however, other types of changes which are still more interesting.

\section{BY COMBINED SELECTIVE CONCENTRATION AND HYBRIDIZATION}

A good example is found in a series of experiments recently carried out, in which $L$. undecimlineata was crossed with $L$. signaticollis of the modified $4 \mathrm{I} 9$ stock, but with these differences. There is a tendency in certain races of L. undecimlineata for the ramous stripe to be broken about one-half its distance from the anterior end. The break in this stripe is fairly common, but is something which is not really fixed in this species, at least by means of any known process; that is, it cannot be rendered a permanent invariable character, but a race can be created in which the 
attribute is present in a higher proportion of individuals than is normal. Such a race was created by means of selection in which about 60 per cent of any generation would have the modification and the other 40 per cent would be without it. These proportions, however, have no significance because those possessing it were just as liable not to be able to transmit it, and those that did not possess it were just as likely to have it show up in their progeny.

The signaticollis stock had further been modified by a selective process in that the amount of black pigment upon the impressed punctations had been considerably increased and by selection the impressed punctations had been brought to a state where they existed in irregular parallel rows. In the extreme of this selected stock which is not constant, the appearance of the elytra is often that of two closely placed irregularly parallel black lines. There is an increase of pigment which finally continued from one punctation to another, making a continuous line, in place of a series of broken dots.

These two stocks, modified by selection, were crossed and gave in the first hybrid generation two types of adults: a mid-type and one like the female undecimlineata type. The mid-type, when inbred, gave four classes of full-grown larvae, $(w h s),(w h S),(y l S),(y l s)$, and from each of these there developed three classes of adults, characteristic of such hybrid operations. In the three classes of adults developed from the (yls) larvae in the second hybrid generations are found a variable number possessed of a combination of the selected characters of the parents; that is, individuals which in normal crosses are of the undecimlineata type, with the elytral stripes present as one single solid band, in this cross have two narrow 
parallel bands. A double-striped condition in place of a single stripe.

Many of the mid-types exhibited the same modification to a lesser degree, especially the break in the band. A female of this double-striped character was mated with an extracted male undecimlineata (a brother), and when inbred there were obtained in the next generation adults with the elytral stripe single, the elytral stripes single and broken, double, double and broken. From these it has been possible, by a process of analysis, to develop the following stable combination: a type in which the larva is white with large black spots upon the back like the larva of signaticollis, but with the ground color white, and with the elytral stripes double in the adult; the same type with the stripes double and broken, and the same type but with the ramous and first costal stripes also broken. Third, the same type of larvae, but with all of the stripes broken. The elytral stripes are extremely invariable and most difficult of modification, but this example shows clearly that by combining in the process of hybridization characters accentuated by selection - and it will probably be true of characters accentuated in any other way-that there is a definite increase in the modification of characters and that they are rendered stable in the constitution of the gamete and might easily take part in the formation of species. Figs. $76 A-G$ show some of the types thus far obtained from an evolution movement initiated by the process described.

In these experiments the modifications are in no wise due to the influence of external factors; in fact, they were carried out under relatively constant conditions, nor is it conceivable that external factors could be productive of such a result. What it is that has brought about these modifica- 

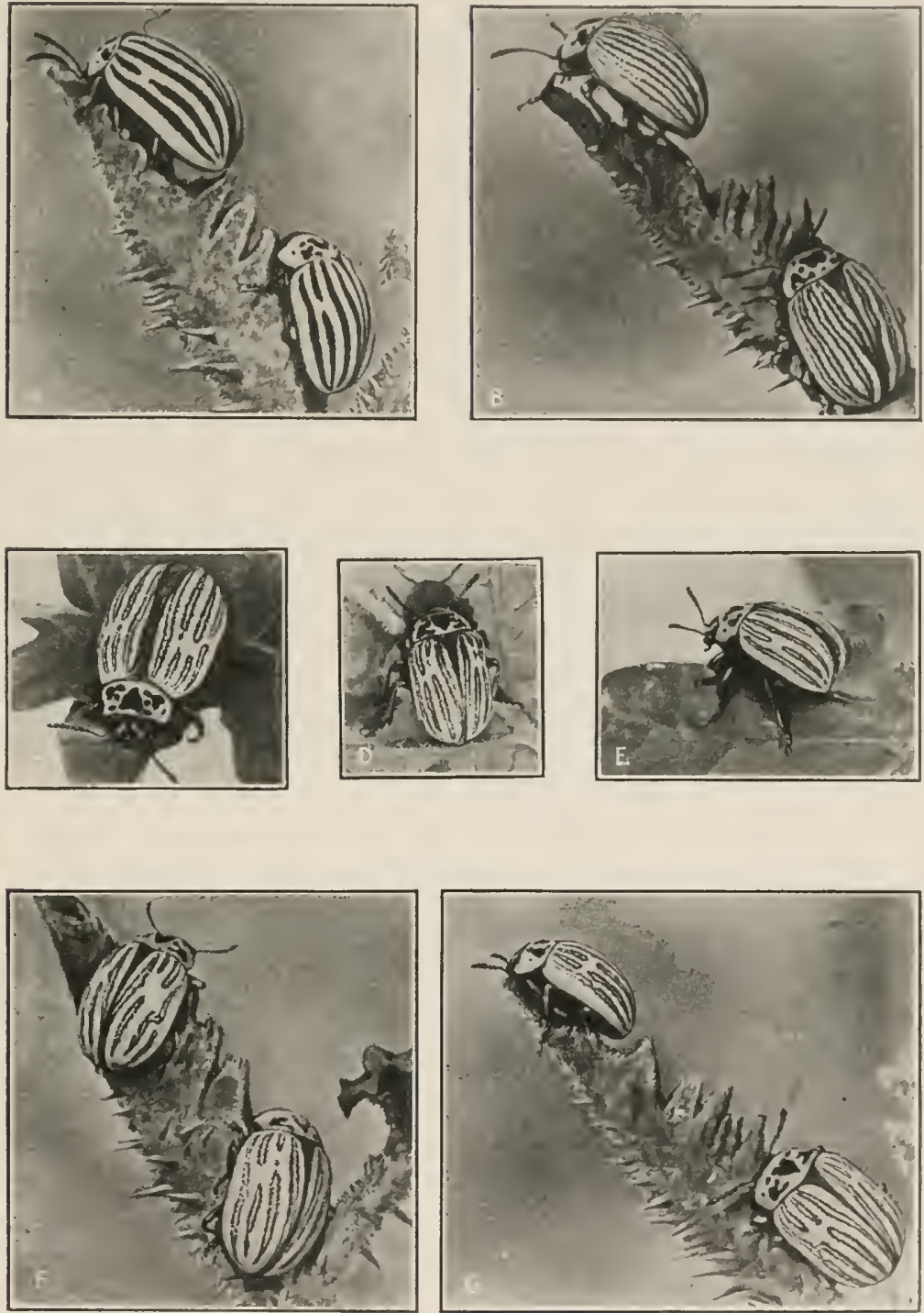

Fic. 76.-To show certain new types that have recently been produced by certain processes. 
tions we have no means of knowing until more is known of germinal constitution. It is clear, however, that by hybridization it is possible to bring about permanent modifications of pattern, and these are not only modifications of color pattern but also of the pattern of the structures, as for example, the punctations, venation, etc. These latter attributes are, by systematists, considered as characters of prime importance, and are made the basis of innumerable systematic distinctions, but in these experiments they are as susceptible to changes, derangement, and de novo variations as are the characters which are based upon color, and color arrangement. In our present ignorance of the nature of the localizing process in any material, either living or non-living, we are left entirely in the dark as to what actually goes on. The experiments, however, suggest the manner in which modifications may result, and these resulting modifications may well be productive of evolution changes either of advancement or regression.

\section{BY SELECTION}

DeVries correctly maintains that the quantitative accumulation of small variations will be productive of a modification which will move rapidly in a given direction until the limit is reached. This can be demonstrated in many plants and animals for a number of characters. The manner in which quantitative accumulation operates is well shown by the following examples:

I attempted by selection to create an albinic race of $L$. decemlineata in two ways-first, by selecting for breeding the most extreme albinic variations found in nature, and second, by creating extreme albinic conditions in experiment and breeding from them. For the first set of experiments the selection was made from numbers of copulating 
pairs found in nature. The selected pairs were kept in separate cages, as were their progeny, the only lumping of material being in the stat tistical treatment of it. The great majority of such pairs and their offspring were not of any interest. Out of 3 I r pairs selected and mated in the years $r \$ 96-1904$, only 26 , or $S_{3}^{1}$ per cent of the total number of pairs tried, were found cajable of transmitting their particular variations. In many of these pairs it was certain that only one of the beetles had the character in transmissible form, so that in $31 \mathrm{r}$ pairs, or 622 individuals, the actual percentage of specimens showing heritable variations was probably not far from 4 or 5 per cent.

Starting from a single pair of albinic inclividuals in which the selected character was transmissible, and following the line of descent from generation to generation, the fact is graphically shown in Fig. $i 7$ that the particular variation of the parent was not only preserved, but carried close to the limit of normal variability of the species, and that by selection the race was changed from one which was variable to one which was relatively invariable - that is, selection resulted in the production of a race of albinic beetles of low variability, which, no doubt, it would have been easy to maintain for a long period of time. From the third generation a selection was made for the parents of the fourth of the most and least albinic individuals, (A) still being the albinic race and (B) the divergent race tending toward the opposite extreme. These two lots of parents gave in the fourth generation two distinct polygons which orerlapped, only in the slightest extent. By continued selection the polygons in the fifth generation did not overlap, and in this generation further division was made of (B) into (B) and (C). These two lines were continued for several generations, diverging from the (A) line, but not far nor rapidly. In the second generation there arose two distinct groups separated by a wide gap (A) and (D), the latter being the exact opposite of the (A) race. This (D) race was propagated, and by selection produced the result shown in the polygons along the line of descent (D), giving in the last generation of the race a group of beetles of almost uniform condition. In all the lines of descent (A), (B), (C), and (D), artificial selection did just what it was found to do in the elements of coloration, namely, it created a race of low variability about the standard chosen which it maintained as long as selection was practiced; but it did not carry the race beyond the normal 


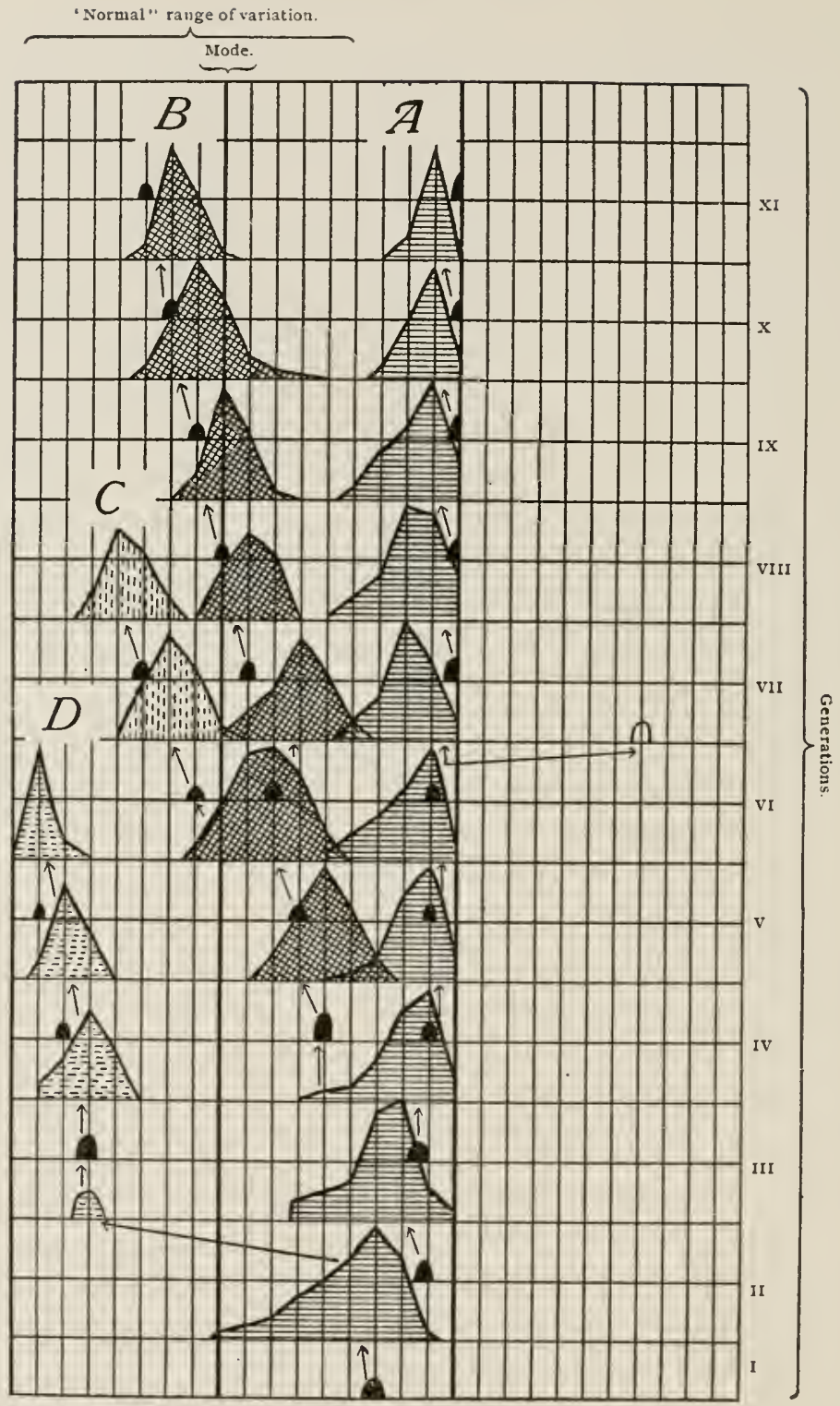

FIG. 77.-Diagrammatic representation of the results obtained in the effort to create albinic and melanic races by selection. Shows also the inability of quantitative accumulations to carry the modification beyond the normal range of variation, and also the rapidity with which races so created revert to the normal standard when the selective influence is removed. 


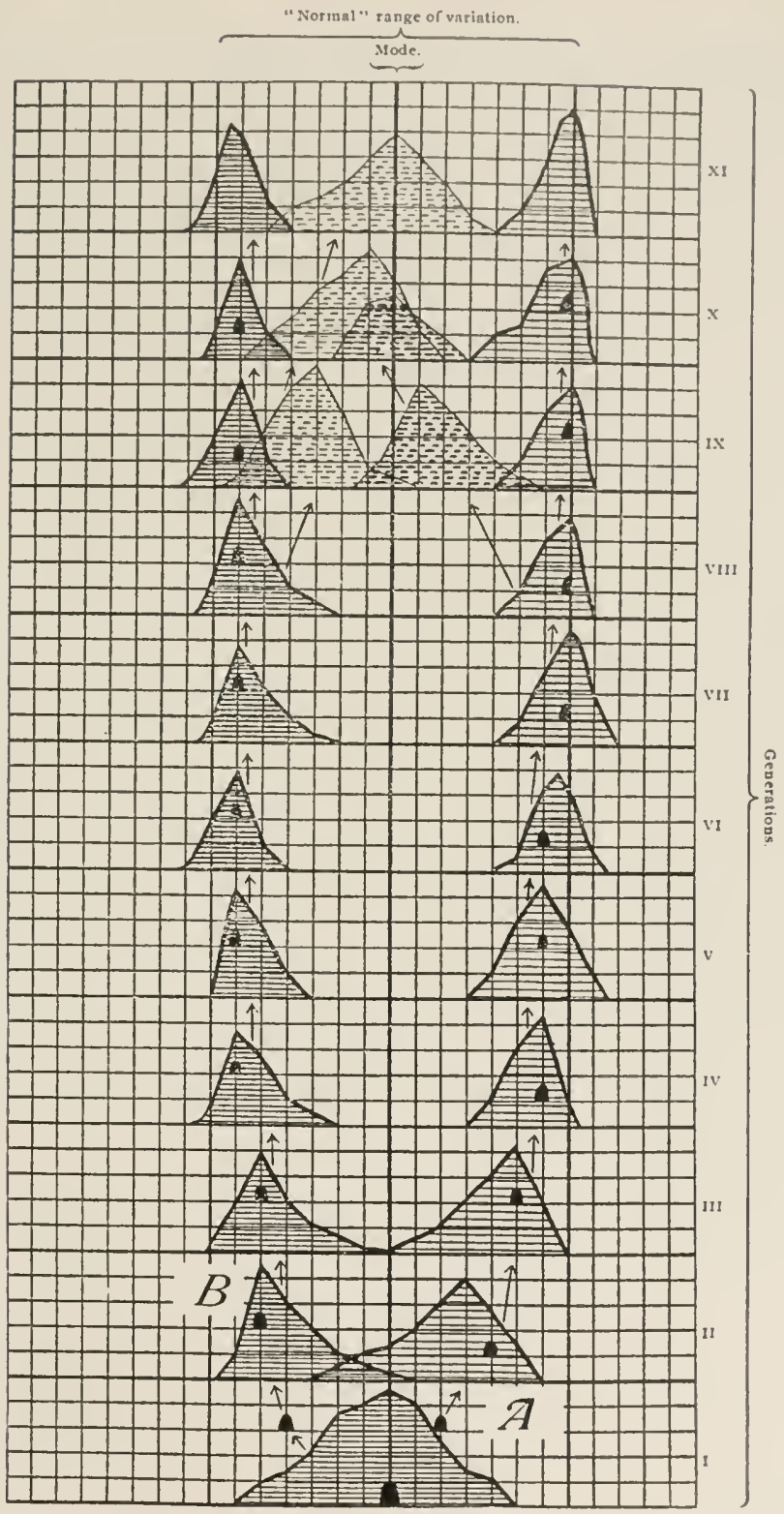

FIG. 78.-Diagrammatic representation of the results obtained in the creation of races by selection of variations capable of transmission. 
range of variation of the species. That is, artificial selection can, as DeVries points out, produce races and maintain them, but its power to develop these races beyond the natural range of variability is yet to be demonstrated.

From the series of cultures represented in Fig. 77, it is shown that it is easy by selection to create races from a species, which would as long as the artificial selection lasted, breed true to the ideal chosen. Another such an experiment and two races breeding true that were produced are represented in Fig. 78 . From the parent generation two selected groups, one melanic (B), the other albinic (A), were taken, and from these, two clearly defined races without trace of intermediate condition were produced. During each of eight consecutive generations slightly variable, light and dark races were maintained. At the end of this time the material was divided and selection was stopped in one group and continued in the other, but the lots were not allowed to interbreed. The removal of the selective factor at once resulted in a regressive shifting of the mode of each unselected race and in increased variability, and this change continued through the eleventh generation, when both unselected lots had moved back to the mode of the species.

These experiments with color characters show very clearly that artificial selection is with transmissible variations a powerful factor and can greatly accentuate any character and maintain it in an extreme condition, but that there are limits beyond which I was not able to modify the characters by this agency. The experiments also show that artificial selection works rapidly, and not, as has been so often assumed, with extreme slowness. True, in experiment I practiced a most rigorous selection, but not more rigorous than that which the natural selectionists believe exists in nature.

The experimental production of general color variations and their preservation by selective breeding give many points of interest. In this I have confined my attention almost entirely to extreme light and dark forms. To produce light forms I have used hot and dry conditions, and for dark forms warm and moist. The experiments herein recorded differ from those already given in that the entire life of the beetles was passed in the conditions of the experiment, and not the larval and pupal stages alone as in the experiments upon coloration. 


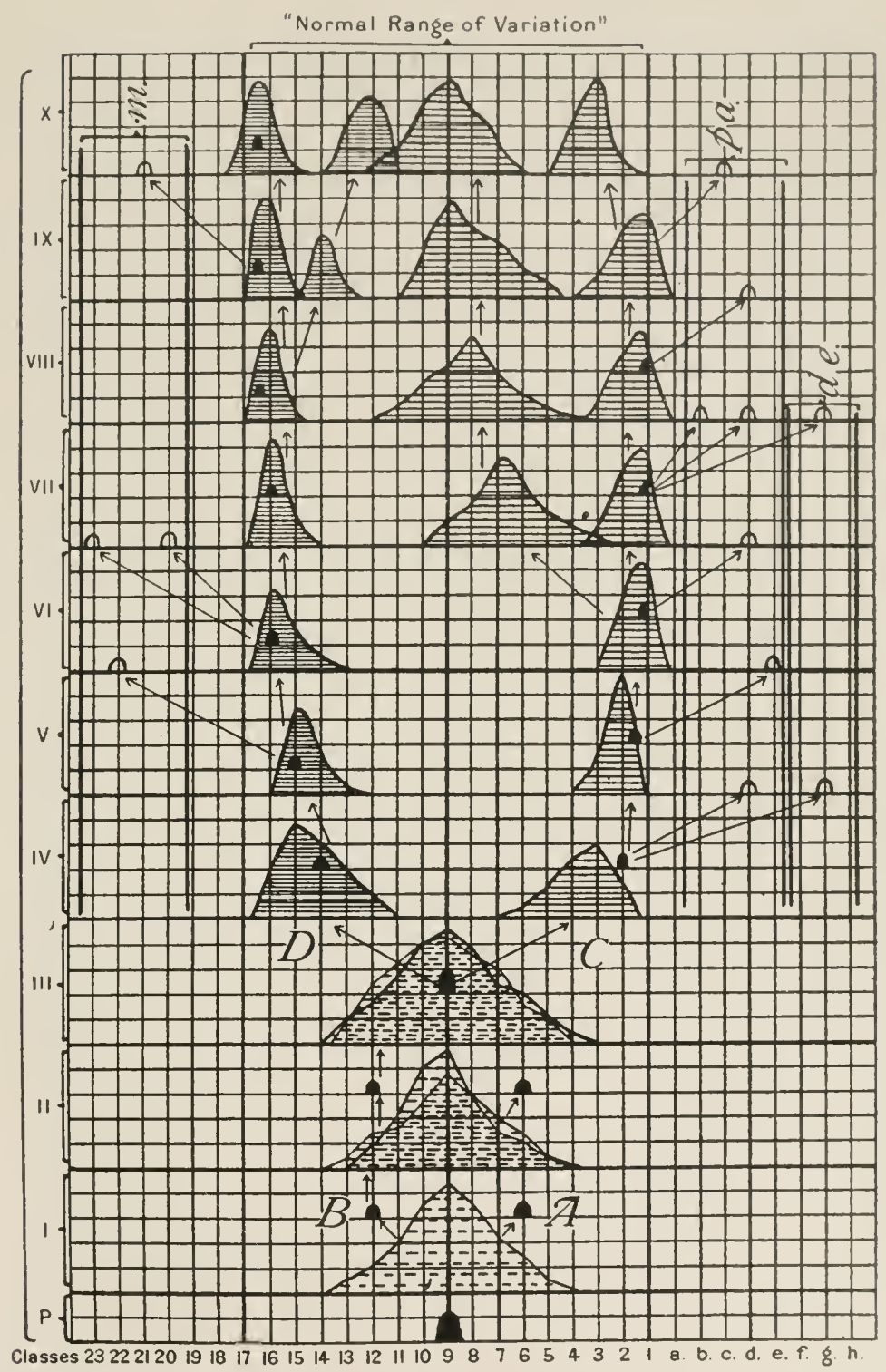

FIG. 79.-Diagrammatic representation of the results obtaineel in the creation of albinic and melanic races by the combined influence of selection and environmental stimuli. These experiments show a difierence from the results shown in some of the former diagrams, in that there are a number of extreme variations produced which apparently are stable in several successive generations; however. when the selective effect is removed the divergent race is seen not to be stable and to revert with considerable rapidity to the mediocre or parental stock. 
In Fig. 79 are brought together in diagrammatic form the data and general history of cultures where both light and dark forms were produced and further subjected to experiment. The black polygons represent the selected groups of parents, the ruled polygons, the offspring. The appearance of "mutants" beyond the normal range of variability is indicated by the small white polygons. .... The series is a complex one, involving processes other than artificial selection and introducing factors of interest which are the key to further experimental study. At present only that portion directly concerned with selection or selective processes need be considered.

In the first, or parent, generation I selected 6 copulating pairs of beetles from the hibernating population, and kept them and their progeny in natural conditions. From the 6 pairs were obtained in the second generation 1,320 mature beetles, and from these, two groups of copulating pairs of ro each (A and B) were selected and reared in the third generation, but showed no modifications as the result of selection. These hibernated, and selections from each lot were reared in the fourth generation, but showed no modification. I now felt sure that the material was pure, that is, normal, and carried no tendencies to appear in divergent extreme variations. Accordingly, from the two series selection was made of as nearly modal individuals as possible, and the two selected lots were mixed and divided into two lots of ro pairs each (C) and (D). These were placed, as soon as possible after emerging, in surroundings productive of dark and light conditions of coloration, and allowed to breed, producing in the fifth generation two distinct lots of descendants, one light, the other dark. These hibernated, and after emerging in the following spring were allowed to breed, when it was found that out of 50 mated pairs $3 \mathrm{~F}$, or 62 per cent, were able to transmit their particular variations in full strength, a huge increase over that found in selections from nature. From each group 5 pairs were selected as the parents of the sixth generation. These gave, as was expected, distinct lots of individuals more melanic and more albinic than their parents, and each also produced individuals differing in many respects from the parent stock, and beyond the usual range of variability. In the five following generations the same thing was repeated, as may be seen from Fig. 79; that is, from each group of 
selected parents there came a general population less and less variable, and a greater or less number of highly divergent forms beyond the normal range of variability of the species. These latter we shall consider in another place.

In this series of cultures a normal parent stock has been subjected to artificial selection aided by powerful environmental stimuli, both having the production of the same end in view. The results, however, were a keen disappointment; the inability to produce by selection and powerful environmental influences a race much beyond the normal limits of variability of the species might easily be taken to indicate the impotency of selection. The ease with which the beetles moved back toward the mode when selection was no longer practiced and the conditions of existence became modal, when joined to the data of place and geographical variations, allows only of the conclusion that while differently colored races and modifications of this organism occur in nature and are produced in experiment by artificial selective processes and local environmental influences, such modifications are limited by the natural limits of variation of the species and persist only as long as the maintaining processes are present and are utterly incapable of existence under adverse conditions, reverting to the species type. That is, artificial selections or local influences are able to modify, and to a certain extent create, races founded upon those variations which are ordinarily killed off by natural selection; but in the creation of such races we really have two forces-a species tendency and a local (or artificial in experiment) - acting against one another, with the result that selective divergence to a certain limit is attained, but beyond that the racial divergence is slow or entirely stopped. When the local or artificial selection is removed the species selective tendency causes a regression to the type of the species. It may be objected that my experiments do not cover a sufficiently long series of generations to have accomplished the result intended, and this may be true; but selection is a poweriul formative factor and works rapidly up to a certain limit, and this has been abundantly proven by plant and animal breeding for fifty years. Why should it not also be able to establish a race on permanent footing with the same rapidity? It is known from the rearing of domestic animals and plants that constant selection is necessary 
to maintain the race. In so far as color characters are concerned, by artificial selection we can easily produce and maintain a race, but cannot establish it as an independent one; it is possible to create isolated races from extreme variations, and by selection keep them isolated, but it seems very difficult to permanently establish them. On the whole, selection would appear as a relatively impotent factor in erolution. Two points should be noted, in passing, namely, the number of highly divergent variations beyond the normal range of fluctuating variation produced in this last series of experiments, and the increased percentage of individuals which show variations capable of being transmitted to the progeny.

It seems to be a well-established fact that by quantitative accumulation, pigment or any other character in the organism can be made to diverge rapidly up to a certain limit, beyond which it is practically impossible to go. More striking, however, is the rapidity with which such races revert to the modal condition of the parent stock when the selective process has ceased. This phenomenon has been characterized as regression toward the mean, and it may represent either one of two processes - an inherent tendency in the organism to revert to the standard, or it may represent a selective process which goes on within the organism. I am of the opinion that the latter is the correct explanation of this, for the following reason: modification by the quantitative accumulation of minute fluctuating variations is always working against what may be termed the natural selective tendency, that is, against those tendencies which surround every organism and which eliminate extremes in each generation through one cause or another, allowing the modal type to persist. Any effort, therefore, to preserve the extreme type comes in conflict with that which serves to eliminate extremes and preserve the modal type. There are two conflicting forces, one which tends 
to preserve the extreme and by rigorous selection to eliminate the mean, and the other which tends to eliminate the extreme and preserve the mean. Rigorous artificial selection makes the preservation of the extreme the more potent of the two, and results in rapid divergence, but not in unlimited divergence, and when this selective process ceases the natural selective process again becomes operative with the result that there is rapid regression to the mean racial standard.

Confusion exists in many records of selection experiments, due to the presence of biotypes or phenotypes, so that the selection is really a process of separating the present elemental forms. In the above-cited experiments only one biotype was present and this was not capable of being changed by selection-quantitative-beyond the normal range. The bearing and necessity of working with one biotype in this selection work and not with a complex of several is well known to all students of genetics at the present day, although not adequately appreciated by practical breeders.

The facts concerning inconstancy and reversion are well known to breeders of animals and plants, who must practice constant selection to maintain the standard of their artificially improved races. Is it possible to produce, by a selective process, modifications which are permanent and which do not revert on the cessation of the selective action, which maintain themselves when brought in contact with the parent species, and which maintain themselves when placed in nature? In selective processes of this kind, amount is a negligible quantity; only pattern arrangement need be considered. In other words, those differences in the constitution of the organism which localize specific characters 
in definite areas can be acted upon by selective combinations to produce permanent results. One example will serve to illustrate this.

Material of $L$. signaticollis Stål, a specific form limited to a narrow habitat in nature, and of low variability in all of its characters, was the basis of this experiment in selection. Compared with other members of the genus with which it is closely related, the variations of $L$. signaticollis are trivial, so that one would on a-priori grounds be certain to regard it as a hopeless task to attempt with $L$. signaticollis studies in experimental evolution by selective methods. By a process of combining variations in the pattern of the pronotum it is possible through a series of generations to create a type of pattern permanent in all respects, and which behaves, when crossed with other patterns, with all the sharp alternativeness of characters found in nature.

The selection was begun in the ninth generation of the stock which had been bred as group cultures and as pedigree cultures and had never shown the modifications which were produced; further, the modifications produced are not known to exist in nature. In the $\mathrm{F}_{9}$ generation, a combination was made of the pronotal pattern, and from this there arose a variable progeny, $\mathrm{F}_{\mathrm{ro}}$. From generation $\mathrm{F}_{\mathrm{IO}}$, matings were made and during generations $F_{I 1}, F_{I 2}, F_{I 3}$, $F_{14}, F_{15}$, and $F_{16}$, etc., the combinations were made from generation to generation, with the end result that there has been developed a permanent strain, which since the $\mathrm{F}_{\mathrm{I} 2}$ of $\mathrm{F}_{\mathrm{t}_{3}}$ generation has remained stable. In this strain the spots are all fused into one solid mass and are carried backward until they completely border the posterior edge of the pronotum, and anteriorly to the anterior edge and laterally, leaving only a small border unpigmented. Such a 
combination is not known in nature, but there has twice appeared in my cultures a somewhat similar variation which could be classed as a sport, which variations, however always failed to perpetuate themselves.

These cultures are able to maintain themselves under the conditions of the vivarium in both-group and pedigree cultures without further attention, or attempts to maintain the race. More interesting, however, is the behavior of these modified forms when placed in nature in their normal habitat, where they have maintained themselves with undiminished attributes, and there has not been the slightest indication of reverting to the ancestral state. When material of this type is crossed with material of the parental stock obtained from the original locality, the modified attribute behaves as a striking dominant alternative characteristic.

Of further interest is the ontogenetic series of events in the development of this modified color pattern. In this instance I know step by step exactly what went into the combination, also what the ontogenetic sequence of erents was in each type of parent, and it is naturally expected that the modifications would present a series of ontogenetic stages one following the other, and that the ontogeny of these modified individuals should rather closely recapitulate the recent events which the organism has gone through in attaining its present state. This is exactly what the organism does not do. Like any well-regulated physico-chemical mechanism it cuts out the non-essential and takes the shortest cut to attain its present end. This short cut, wherein stages which it passes through in its phylogenetic derelopment are left out, and where many characters are lost completely, shows that in this instance a modified patterm has 
arisen by an accumulative process, giving all the same permanence and strength of character as is found in what are called natural species or characters.

The same process has been applied to other forms and attributes with identical results, the only limitations being such as are imposed by the physical constitution of the organism itself, or of the part in which the modification is being carried on, so that a character might become after a time decidedly injurious in the economy of the species and therefore come under the operation of natural elimination. This pattern, however, is a neutral character and the only limitation is that imposed by the physical constitution of the part and there is apparently no direction in which the modification must never go.

In another series derived from the original stock, modifications were carried out in the reverse of this one, producing a stock in which all of the spots were reduced to round simple areas with absolutely no tendency to fusion or breaking up of the spots into many tributary rows. From these experiments two points are clearly demonstrated: First, it is shown that a selective process may permanently modify one of the most inflexible of characters, namely, pattern, and this modification may even greatly exceed any of the variations known in the species. This selective process is different from the quantitative accumulation which has been employed by plant and animal breeders. It is not a process of hybridization, but is analogous to the processes which a chemist would use in synthetizing a complex compound, adding to it first one thing, then another, subtracting from this a product, then adding to it some more, until the end product is totally different from the original material. It is a process of synthesis and not of accumulation. 
The second observation has a bearing upon the current theories of biogenesis and orthogenesis, showing the distinct failure of this modified organism to repeat in its ontogeny the stages which it has recently passed through in its phylogenetic development. It has long been maintained, from a paleontological standpoint, and from study of the ontogeny of an organism, that the stages passed through represent a recapitulation of the more essential stages of its recent development. Jackson on paleozoic echini, Hyatt on ammonites, and Beecher on brachiopods have shown that there is a constant dropping of the earlier and more primitive stages in the ontogeny of a species, and a reduction of the later stages. There is of course a large amount of permanent truth in the view obtained from these paleontological studies, that the organism recapitulates its phylogeny, at least to a greater or less extent, but it is equally true that the literal interpretation of this--the utilization of the ontogenetic stages as a test in determining relationships and direction of evolution-is futile.

It would be difficult to interpret a case like the one given on any other basis than that used-that the conditions found represent the best adjustment which the physicochemical mechanism could possibly achieve in order to attain a definite end. To do this, instead of following an irregular and complex path, it cuts straight across, eliminating minor steps which have played a phylogenetic rôle but have no part in the achievement of the final end result in the ontogeny of the parent stock.

It has been maintained that evolution is irreversible and that once started it must continue to the end. It is difficult to conceive of any reason why this should be so unless there be assumed the existence of an inherent force 
or cause driving organisms toward a series of goals, which, when passed, represent the final goal of that particular race. If this conception were true, then there would exist in organisms a state totally unlike that found elsewhere in nature. In no physical or chemical phenomena does any such condition exist, but rather, an array of substances which can be combined and recombined in an almost infinite series of combinations, which can be built up to complex aggregations and with equal facility reduced down to the atomic groups from which they came. In other words, by a selective synthetic process it is possible to create or synthetize, and likewise, by a selective analytical process to analyze, and the irreversibility of evolution exists only in the dogmatism of some essayists, and not in the materials of nature.

To what extent the selective process is operative in the production of variation in nature is unknown. It is not probable that in nature there would exist the arrangement of variations which I brought about in experiment, but in nature I should rather look for a very decidedly haphazard process, bringing about chance combinations which would produce this or that result, and these chance combinations might any two of them combine and produce a third chance combination which would stand apart from the parent species-the product of the two others, but if found in nature it would be described as a "mutation." What rôle these variations, and I am convinced that they are potent factors in evolution, really would have in the general evolution of organisms and in the development of species, is unknown. It is not inconceivable that a variation might thus arise which, behaving as a strong dominant or as a dominant heterozygote, could increase in numbers and 
after a time replace the parent species to a considerable extent, if not completely.

\section{Discussion-Summary}

Probably no question in biology has received more attention, and certainly few are so little understood, as the method whereby those variations which are productive of permanent change arise and become incorporated into the germinal constitution of the race. If the possibility of variations arising in a manner like that assumed in the neo-Lamarckian conception be admitted, it must also be admitted that there is at present no critical evidence of any such method of origin. As far as experience warrants a conclusion, there is at present no escape from the general proposition that all variations that are productive of permanent germinal changes, arise primarily in the germ and appear secondarily in the soma. It must be understood that by this proposition it is not asserted that it is the only possible conception, but that it is the only one concerning which there is at the present time any definite proof.

Knowledge concerning the germ cells, which are the germ plasm or else the carriers of it, is largely anatomical in character, derived from studies in cytology and in the main is one sided, incomplete, and has been too much directed to the study of the chromosomes. That this is the condition is not strange when one considers the wonderful regularity with which the chromosomes are divided between the daughter cells, and the precision and regularity of the process immediately preceding and accompanying fertilization. These phenomena, so fundamental and common to all organisms, have impressed biologists profoundly and very naturally the opinion arose that the chromosomes were the 
bearers of that which conditions the characteristics of the subsequent generation. Recent cytological studies, however, have demonstrated that the early conception of equality in the distribution of the chromosomes is not entirely true, but that there is a regular and unequal distribution of the chromosomes which occurs in many, if not all animals, producing in some two classes of ova, in other instances two classes of spermatozoa.

These chromosomal differences in some unknown manner are now generally admitted to be associated with the determination of sex; hence the "accessory chromosomes" which go to make chromosomal differences in the germ cells are regarded by some as sex determinants. That they are sex determinants in the sense in which "determined" has been used is not proven. The results obtained by Morgan in Phylloxera seem crucial and show that the extra chromosomes are an accompaniment of differentiation, and not the cause thereof. At any rate it is definitely proven that the existence of the accessory chromosomes represents a definite difference in the qualities and constitution of the germ cells, and thus gives in gametogenesis germ cells differing from one another by sharp alternative differences. There is no a-priori reason why the same may not be true of attributes and qualities in the germ cell which are not capable of observation by present cytological methods.

In the last decade the work of many investigators, but especially that of Conklin, Lillie, Morgan, Driesch, and Wilson, has shown that the germ cells are in reality highly complex structurally, possessing a definite organization and polarity, with a distribution of various elaborated substances which are individually more or less necessary to the proper development of particular parts of the future embryo. 
It is clearly shown that in the germ cell before fertilization there is a fundamental symmetry, polarity, from which there arise during ontogeny the symmetries and arrangements existing in the developing individual. This symmetry, as far as there is any evidence, continues back through all the cell generations which arise during gametogenesis, and on the basis of what is known of development in the germ cell, it seems to be a symmetry directly derived from the parent zygote. In other words, in the continuity of the germ cells from generation to generation, there is a continuity of the symmetries and physical constitution characteristic of the germinal material, a continuity of germinal organization. It seems clearly established, especially by the work of Lillie and Morgan, that there is a fundamental background, or matrix, in the germ cell in which the original symmetries are expressed. For the present the entire germ cell must be regarded as the germinal material or germ plasm, and our problem in the investigation of the origin of germinal variations is to discover by what methods changes in the germ cells are brought about. This is one of the most difficult problems confronting biologists, and one which is possibly incapable of solution, at least in the near future, and perhaps may forever remain unsolved.

The chemical changes which go on in the living substance, especially those which follow in the metabolism of organisms, have been roughly outlined, but these metabolic processes, while they may result in the elaboration of products to be incorporated into or removed from the organism in one way or another, do not possess any capacity for carrying on their operations excepting in the living mass. Moreover, we understand. in a broad way the mechanical 
and physical principles involved in the passage of fluids from one part to another or to the outside, and something of the rôle which katalytic changes play in organic activities. The time has not come when this information can be directly applied to the problem of the chemical nature of germinal changes.

As regards form and pattern, the biologist is confronted in organisms with the same difficulties as are presented to the physicist and chemist to explain form and pattern in nonliving substances. No physicist would presume to say what it is in the composition of a crystal which makes so definitely for a specific form of face, hardness, and optical properties. The problem is the same in both living and non-living substance, and the forces which determine form and pattern seem to be properties of the entire mass and are not localized, and are probably produced in organisms, as in nonliving masses, by the sum total of the interactivities of the mass at the moment of observation.

In every part of the organism there is a symmetry which is the outgrowth of the original symmetry from which the organism developed. Variations in the secondary or later symmetries may be large, but almost never are there variations in the fundamental symmetries which distinguish phyla. Evolution is mainly concerned in the problem of the formation of species or attributes with variations in these secondary symmetries and patterns. I have shown how certain of these symmetries may be modified.

Not the slightest clue of what it is that is modified in the germ cells of a particular species as the result of any modifying process has been recorded, and the usual attempted explanations are based upon assumed a-priori conceptions, usually atomistic in character, on the order of Darwin's 
provisional hypothesis of pangenesis, the id-determinantbiophore fabric of Weismann, or the pangene complex of DeVries. Much has been written concerning the uselessness of such conceptions as explanations; much has been written in their favor, and the only truthful statement possible is that there is no evidence for their existence. The important contribution is the experimental evidence showing that the symmetries and patterns in organisms are definitely modifiable as the result of synthetic and other processes which may be carried on under observation, and which achieve definite results. In most instances thus far recorded results are achieved rapidly, and the end is not postponed or approached in a halting, zigzag manner, but the modification is in appearance definite, precise, reminding one of the regular and precise operations seen in chemical and physical processes.

There is an interesting analogy between the processes which may be carried on in organisms in modifying the symmetries by combining definite characters, and those which may be carried on in crystallography, as for example, where certain impurities may be introduced into the crystallized form and may definitely alter the attributes of the crystal, such as shape, color, hardness, specific gravity, etc. That the parallel is an exact one there is no evidence, but it is at least suggestive in that both the results occur with sharp alternativeness which is so characteristic of chemical and physical operations.

It does not seem probable that germinal modifications in form and symmetry arise through the changes in particular chemical constituents within the germ cell, as, for example, a particular katalytic agent, or the rearrangement of the side chains in some complex molecule, because, as far 
as is known, these are superficial relations and are not of themselves concerned in establishing the fundamental symmetries and activities which seem to be in the main conditioned and controlled by the colloidal matrix which underlies all visible structure. The only safe statement which can be made at present is an acknowledgment of our ignorance of what the changes are in the germ cell which are productive of new arrangements in form and symmetry. However, it is experimentally proven these fundamental relations can be altered by one process or another, thus giving methods of inducing changes and of discovering what changes are possible, both as regards the limits of change, direction, rate, etc., and this knowledge may be of great practical value even though the underlying physicochemical operations are still undetermined and possibly unknowable.

In the modification of characteristics which are directly conditioned by chemical activities, as, for example, color, there seems a greater possibility of attaining at least a general idea of the processes involved in the germ cells in producing permanent changes. Pigments, throughout the organic world, are pretty generally the result of metabolic processes and are probably in most instances the result of the oxidation of various cleavage products which have themselves been formed by the breaking down of more complex substances within the cell. Many of these chromogen substances are possibly waste materials in the organism, which perhaps could not be further utilized in the economy of the animal, so this method arose of converting them into more or less harmless substances and depositing them in places where they would be least inconvenient to the organism. This old conception of the character of coloration 
meets with little favor today, because there is too much good evidence to show that even under adverse conditions the development of pigment takes place, where the organism expends in developing the pigment, material, and energy which it needs to carry on activities vital to its existence. The pigment substances which develop in connection with pathological growths and the development of pigment even in the face of starvation are all indicative of the deep-seated nature of pigment formation in organisms.

The capacity to produce a given pigment is as firmly a part of the germinal constitution as are structural characters, which, by some at least, are regarded as the only attributes worth considering. It is well known that in all organisms there is in nearly all cells at least the possibility of producing from the ordinary breaking down of the substance of the cell, materials which can serve as the chromogen base for the elaboration of various kinds of pigment, but this aspect of the subject has been considered in an earlier part of this paper.

The question in the production of germinal modifications of the pigment-forming capacity in organisms is, what is it in the germ cell that is modified? There is little to warrant an assumption that these phenomena are based upon representative particles or upon individualized entities of any sort, and I doubt if many investigators really attribute to pangenes and biophores the capacity and importance which some writers who are antagonists to the method of expression seem called upon to believe.

Perhaps the best evidence in this direction is that derived from the studies in inheritance by the Mendelians. Their treatment of the subject of color inheritance has shown clearly that there is something in alternative char- 
acters which, when a definite array of conditions are brought together, produce a definite color, and when another array is brought together a different color results, or perhaps no color.

At the present time there is no evidence that in the cells there is incapacity for the production of pigment, or incapacity for the production of either chromogen or enzyme. The only evidence is that the pigment does or does not appear when germ cells derived from parents of a certain character are combined. In the minds of some it follows from this that something is lacking in the way of a specific activity; in the minds of others it is due to the fact that a sufficient quantity of one or the other of two necessary substances is not present. Still another explanation is that there is lacking strength or energy to produce the one or the other.

Further, the situation can be explained by adopting the idea of inhibitors, activators, etc., which would inhibit the appearance of pigment in one case, and then, by the inhibition of the inhibitor permit the appearance of pigment in another case. Much fine evidence exists from the work of the neo-Mendelian hybridologists that factors, determiners, accelerators, and inhibitors exist and can be subjected to experimental tests; however, to attempt to explain observed conditions by asserting that the organism is incapable of producing the requisite amount of chromogen or activator is really no explanation, because it is well known that a minimum amount of activator may convert an almost unlimited amount of chromogen into a color-forming substance, provided the substance produced as a result of the katalyzing process is removed with sufficient rapidity so as not to impede the process. This is a well-known principle 
in all katalytic action. It does not seem a plausible explanation of germinal color variation to attempt to place it upon a quantitative basis, in view of the well-known facts of katalysis. Likewise, explanation of the situation as being due to varying germinal strength, energy units, energy, etc., does not aid, and only confuses a situation already sufficiently confusing. If by strength is meant strength of katalyzer, there again a weak agent may produce a relatively large result if advantageously placed and given a sufficiently long time in which to act to bring about a definite result, and a relatively weak agent may, under advantageous conditions, convert a relatively enormous amount of chromogen into pigment-forming substance. It would be of interest to know the chemical constitution of some of the attributes; in the case of albino animals, for example, is the chromogen present, and is the oxidizing agent absent or vice versa? It seems improbable that the oxidizing enzymes should be entirely lacking, and it may be true that there is a specificity in these enzymes, as has been suggested by various authors, and a specific enzyme might well be absent, and in its absence there would be no production of color.

As far as I am able to get at the processes involved in the production of color variations by means of incident physical and chemical factors, it seems that the change is one which involves the entire mechanism of the cell, and is not resident in any particular part thereof. By experimental means I have produced a modified condition of coloration, and in this the interactions of chromogen and katalyzer are conditioned in their appearance and interaction during ontogeny by the mass in which they are, and are entirely dependent upon the capacity of the mass to retard, accelerate, or extend the rate and time of action, 
or to remove the by-products which would inhibit the katalyzing action which would normally go on. Under the modified conditions there may be produced no greater alteration in the germ than a reversed action of some enzyme, and although the same enzyme may be present, its reversed action is such that the pigment-forming substance is not formed, but its activity is directed to other activities than to building pigment-forming substances. The same enzyme may again be reversed in its direction either by combining with some other substance, or by being acted upon by some incident force, and this reversal of action in the enzyme may therefore produce the formation of color substances. It does not of necessity follow that the changes induced are at all concerned with the substances which actually themselves form the pigment. For example, in germinal modifications which have been produced, where the color is diminished in intensity, there may be identical amounts of chromogen and enzyme, but the changes may be due to changes in other factors which are necessary to the color formation by removing from the field of operation certain inhibiting by-products.

It is possible that a profitable point of attack upon these problems lies in this direction. The chief difficulty, however, is the small size of most germ cells, and in those of large size the existence in the egg of a large amount of stored food supply effectually inhibits investigation. It is probable that for the present interpretation of the process of germinal change must be more by analogy than by actual physical and chemical analysis.

In the experimental production of modifications of the germ plasm two definite ideas as to the nature of the change have been expressed. MacDougal is of the opinion that 
in many of his derivatives all of the attributes of the organism were modified each more or less independently of the others. I have expressed the opinion that, as far as my observations go, one character is in the main most modified, and then a greater or less array of lesser characters are less modified in correlation. There is at once a fundamental difference between these two expressions of experience, a difference which may be due to the difference between plants and animals, but I do not understand how all the attributes of an organism can be modified at one time by incident forces. MacDougal says, "The induced forms in plants show many" new qualities of fairly equal importance as far as such things may be estimated, and these might be independent of each other."

I do not, at least in animals, feel competent to determine the equality of characters, and thus far I have never seen examples, where any considerable array of characters were modified equally. As a matter of fact, I have no basis in experience which would enable me to decide whether characters were modified equally or not.

If one accepts a particulate conception of the constitution of an organism as a true expression of organic constitution, the modifiability of characters as expressed by MacDougal would be the natural result. I have thus far seen no evidence of such a condition, at least in the materials under my observation, and I am therefore compelled to regard the organism as a whole in the sense that it represents a state of stability which is achieved and retained by a mass of matter under a given set of conditions. The combined elements are not independent and do not exist in the organism as individualities, but as component parts of the whole, as long as they comprise a part of that particular 
substance, even though they are capable of unlimited metathetic change in heredity.

There is another point upon which MacDougal and I differ. MacDougal says, that "his new plants do not hybridize freely, if at all, even when grown with branches interlocking with the parental type," which differs from the conditions which I have found, where intercrossing occurs freely with the parent species. Comparison is difficult between organisms so widely separated as those used by MacDougal and myself, and it is entirely possible that the results of particular processes may result differently in different organisms.

The results obtained by Gager through the use of radium upon different plants, the modifications of Sempervivum by Klebs, the changes in yeasts and bacteria, etc., can hardly be incorporated at the present time in this discussion in that they have not been carried far enough. Gager's opinion that the inheritable results obtained by him were possibly due to a disturbance in the chromosome complex, is simply an explanation based upon an old morphological conception. It has not yet been shown that specific chromosomes are endowed with specific characters resident in them, and until that point is decided definitely the explanation of germinal variations on the basis of chromosome behavior must stand aside.

In animals the experiments of Kammerer, Pshibram, Woltereck, Morgan, and the older experiments of Fischer, Standfuss, Weismann, and others, do not furnish data for a discussion of and far less for a solution of the problem. However, an important point is established by all of these investigations which show uniformly that the germ cells are most susceptible to the influences that produce germinal 
changes immediately before and during maturation. 'This period is known to be a critical one in the life of the germ cell, and is a time when many processes are in progress, and when there exists a delicate balance susceptible of being permanently upset. There is evidence in some of my experiments to show that earlier periods in the life-history of the egg are susceptible to stimuli and produce at these earlier periods more profound modifications than are produced later, but are more difficult to induce. During the growth period there is a constantly increasing elaboration of potentialities, with an increasing array of substances and symmetries, and a slight change in this primary constitution might result in large end results.

It is evident that the problem of germinal change is one of difficulty, and involves more of indirect than of direct methods of investigation. There is little reason to expect that present biochemical methods can give a solution, but they may give valuable suggestions for further indirect investigation. It seems not improbable, however, that this problem, like so many others in biology, must await the solution of the larger question of what life is before it will be possible to express in exact terms the nature of germinal changes. Our present status, with several methods of production and much knowledge of the behavior of induced germinal changes available, is a basis from which great advances in knowledge and in operation may reasonably be expected. 

CHARLES BENEDICT DAVENPORT

Station of Experimental Evolution, Carnegie Institution of Washington 



\section{CHAPTER VIII}

THE INHERITANCE OF PHYSICAL AND MENTAL TRAITS OF MAN AND THEIR APPLICATION TO EUGENICS

The general laws of heredity have already been fully explained. But every person has a different way of expressing them and a little repetition will do no harm; and so, very briefly, I will recapitulate the principles of heredity.

First of all, we find useful the principle of the unitcharacter. Whether it be ultimately accepted or discarded, it is useful today, and so we accept it as a guiding hypothesis. According to this principle characters are, for the most part, inherited independently of each other, and each trait is inherited as a unit or may be broken up into characters that are so inherited.

Next it must be recognized that characters, as such, are not inherited. Strictly, my son has not my nose, because I still have it; what was transmitted was something that determined the shape of his nose, and that is called in brief a "determiner." So the second principle is that unitcharacters are inherited through determiners in the germ cells.

And finally, it is recognized that there really is no inheritance from parent to child, but that parent and child resemble each other because they are derived from the same germ plasm, they are chips from the same old block; and the son is the half-brother to his father, by another mother.

These three principles are the three cornerstones of heredity as we know it today, the principles of the inde- 
pendent unit-characters each derived from a determiner in the germ plasm.

It is my agreeable task to show in how far the known facts of heredity in man are in accord with these principles. I may say at the outset that I have no doubt that all human traits are inherited in accordance with these principles; but knowledge proceeds slowly in this field.

As a first illustration I may take the case of human eye color. The iris is made up of a trestle work of fibers, in which are suspended particles that give the blue color. In addition, in many eyes, much brown pigment is formed which may be small in amount and gathered around the pupil, or so extensive as to suffuse the entire iris and make it all brown. It is seen, then, that the brown iris is formed by something additional to the blue. And brown iris may be spoken of as a positive character, depending on a determiner for brown pigment; and blue as a negative character, depending on the absence of the determiner for brown.

Now when both parents have brown eyes and come from an ancestry with brown eyes, it is probable that all of their germ cells contain the determiner for brown iris pigmentation. So when these germ cells, both carrying the determiner, unite, all of the progeny will receive the determiner from both sides of the house; consequently the determiners are double in their bodies and the resulting iris pigmentation may be said to be duplex. When a character is duplex in an individual that means that when the germ cells ripen in the body of that individual each contains a determiner. So that individual is capable, so far as he is concerned, of transmitting his trait in undiminished intensity.

If a parent has pure blue eyes, that is evidence that in neither of the united germ cells from which he arose was 
there a determiner for iris pigmentation; consequently in respect to brown iris pigmentation such a person may be said to be mulliplex. If now such a person marry an individual duplex in eye color, in whom all of the germ cells contain the determiner, each child will receive the determiner for iris pigmentation from one side of the house only. This determiner will, of course, induce pigmentation, but the pigmentation is simplex, being induced by one determiner only. Consequently, the pigmentation is apt to be weak. When a person whose pigment determiners have come from one side of the house forms germ cells, half will have and half will lack the determiner. If such a person marry a consort all of whose germ cells contain the determiner for iris pigmentation, all of the children will, of course, receive the iris pigmentation, but in half it will be duplex and in the other half it will be simplex. If the two parents both be simplex, so that, in each, half of the germ cells possess and half lack the determiner in the union of germ cells, there are four events that are equally apt to occur: (I) an egg with the determiner unites with a sperm with the determiner; (2) an egg with the determiner unites with a sperm without the determiner; (3) an egg without the determiner unites a sperm with the determiner; (4) an egg without the determiner unites with a sperm without the determiner. Thus the character is duplex in one case, simplex in two cases, and nulliplex in one case; that is, one in four will have no brown pigment, or will be blue eyed. If one parent be simplex, so that the germ cells are equally with and without the determiner, while the other be nulliplex, then half of the children will be simplex and half nulliplex in eye pigment. Finally, if both parents be nulliplex in eye pigmentation (that is, blue eyed), then none of 
their germ cells will have the determiner, and all children will be nulliplex, or blue eyed. I have gone into the inheritance of eye color at some length because it serves as a paradigm of the method of inheritance of any unit-character.

Let us now consider some of the physical traits of man that follow the same law as brown eye color, traits that are clearly positive, and due to a definite determiner in the germ plasm. And first, I may refer to hair color.

Hair color is due either to a golden-brown pigment that looks black in masses, or else to a red pigment. The lighter tints differ from the darker by the absence of some pigment granules. If neither parent has the capacity of producing a large quantity of pigment granules in the hair, the children cannot have that capacity, that is, two flaxenhaired parents have only flaxen-haired children. But a dark-haired parent may be either simplex or duplex; and so two such parents may produce children with light hair; but not more than one out of four. In general, the hair color of the children tends not to be darker than that of the darker parent. Skin pigment follows a similar rule. It is really one of the surprises of modern studies that skin pigment should be found to follow the ordinary law of heredity; it was commonly thought to blend. In crosses between Negroes and Caucasians, such a blend was stated to occur, and it was believed to be permanent, so long as the hybrids were mated together. Actually, the method of inheritance is like that of hair pigment, the skin color of the children rarely much exceeds that of the darker parent. There are stories of two white parents having black-skinned children, and if these are true they constitute striking exceptions to the general rule. The inheritance of skin color is not dependent on race; two 
blondes never have brunette offspring, but bruncttes may have blondes. The extreme case is that of albinos with no pigment in skin, hair, and iris. Two albinos have only albino children, but albinos may come from two pigmented parents.

Similarly, straight-haired parents lack curliness, and two such have only straight-haired children. Also two tall parents have only tall children. Shortness is the trait: tallness is a negative character. Also when both parents lack stoutness (are slender), all children tend to lack it.

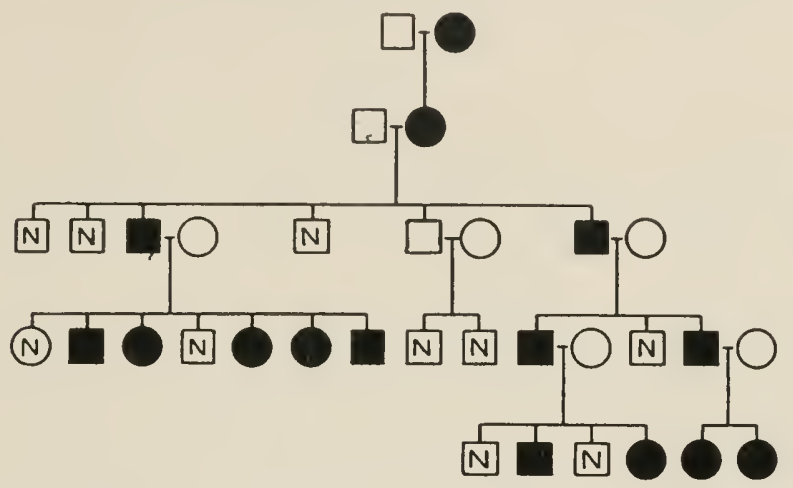

FIG. So.-Inheritance of monilithrix-a positive character. Black symbols represent affected individuals.-ANDERSON.

We may now consider briefly the inheritance of certain pathological or abnormal states, to see in how far the foregoing principles hold for them also. We shall find that sometimes the abnormal condition is positive, due to a new trait; but sometimes, on the contrary, the normal condition is the positive one and the trait is due to a defect.

Among conditions due to a new determiner may be mentioned a beaded peculiarity in the form of the hair, called monilithrix. Affected persons tend to have affected offspring (Fig. So). Two unaffected parents do not ordinarily 
have affected children (in the illustrative case nothing is known about one of the two parents). When one parent only is affected about half of the children are affected, since each affected person is simplex.

Again, some family strains exhibit imperfection of hair or even baldness associated with imperfect nails (Fig. 8I). Here there seems to be a determiner that stops the development of these organs of the skin. Normal persons are without this determiner and so cannot have affected children.

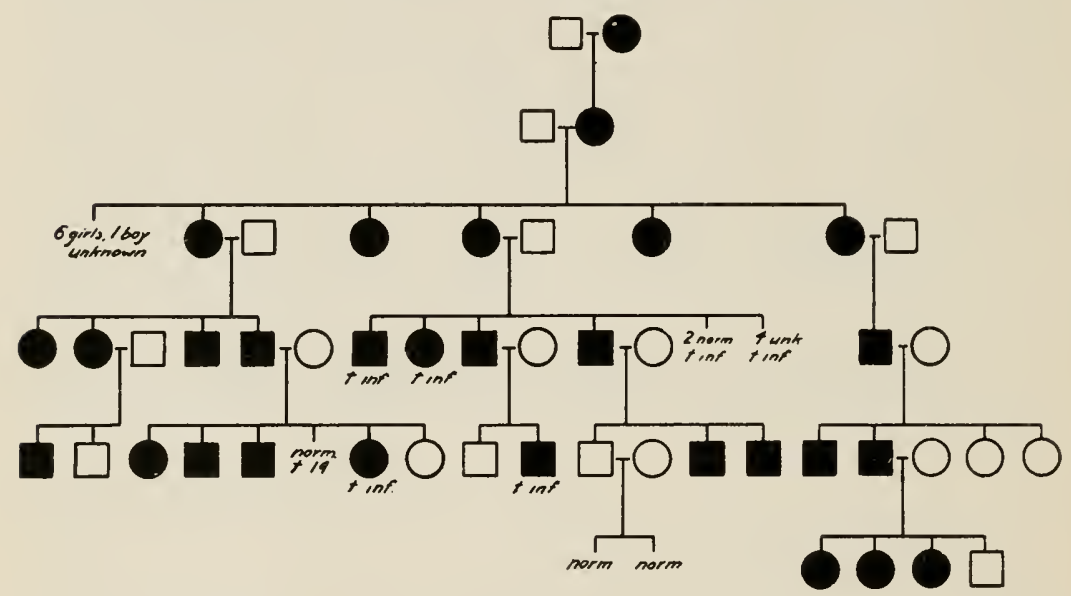

FIG. 8r.-Pedigree of a family with poorly nourished nails and hair (black symbols).-Nicolíé et Halipré.

Still another peculiarity of the skin is due to a positive character. This is a thickening of the palms of the hands and soles of the feet (Fig. 82). Here all, or half, of the children of an affected parent are affected, but normals of the strain, who marry outside of the family, will have no thick-skinned children.

In some persons the color of the hair of the head is not uniform, but there are patches of white hair in the midst 
of a prevailing brown head of hair. This spotted condition is due to a positive factor, just like spottedness in mice. From a spotted parent at least half the offspring are spotted;

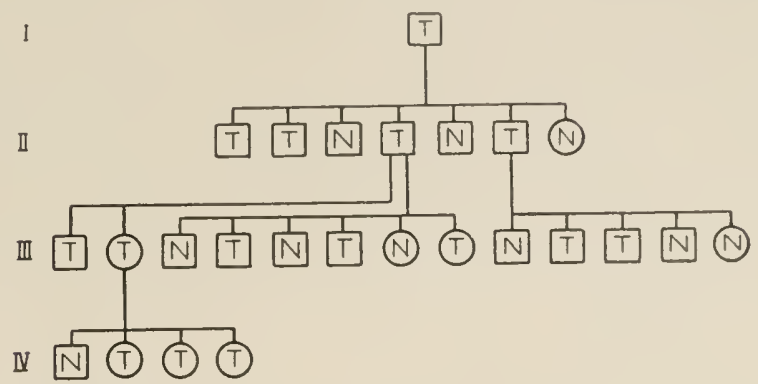

FIG. 82.-Pedigree of a family with tyloses (T). Note that all affected persons have at least one parent affected.-UNNA.

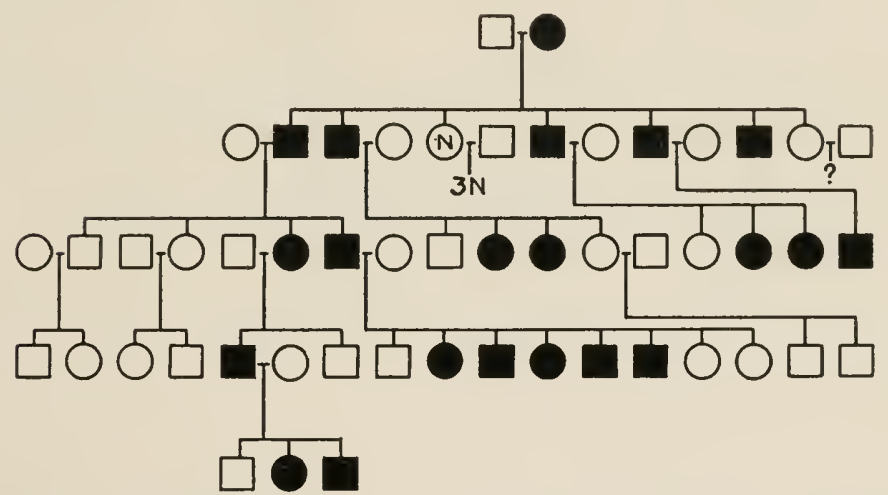

FIG. 83.-Pedigree of faulty enamel of teeth. This peculiarity appears only in the offspring of an affected parent, consequently it is a positive trait.-TERAER.

but a person with uniform coat belonging to the spotted strain will have no children with the white patch.

Probably the same law is followed in the case of families without teeth and also in families with faulty enamel of the teeth (Fig. $8_{3}$ ). Apparently there is a determiner that 
partially or fully prevents the development of the tooth germ.

Some peculiarities of the eye are clearly inherited. Thus the condition in which the lens of the eye becomes clouded and opaque seems to be due to a positive determiner, and this is most clearly seen when cataract appears in middle life. For, all cases of presenile cataract appear in the offspring of affected persons, and two unaffected persons probably never have descendants with cataract (Fig. 84).

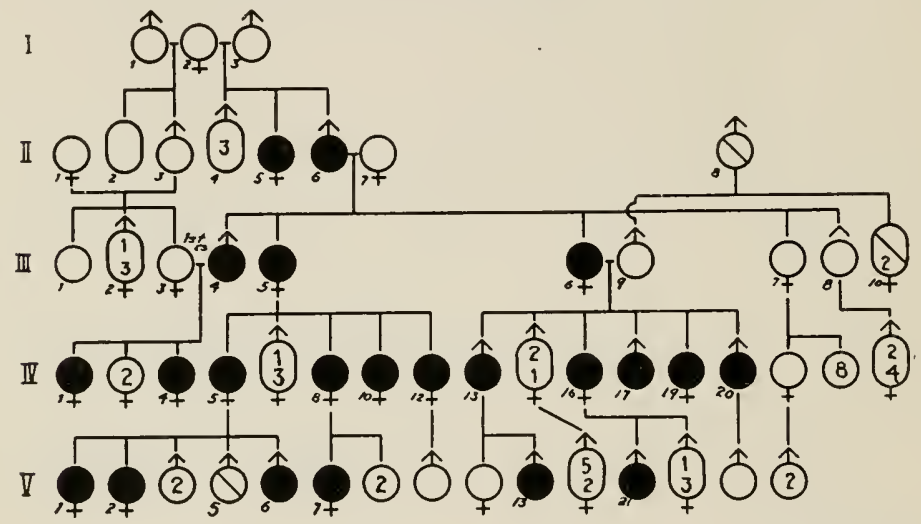

FIG. 84.-Pedigree of a family with presenile cataract (black symbols). Numbers in circles indicate unaffected individuals. Cataract in offspring of afiected parents only.

The same law holds for a peculiarity of the eye such that the affected person cannot see by weak light, such as the light of lamp, electric lights, etc. (night blindness). There is clearly a positive determiner, since affected persons have affected offspring, but if the parents are not affected (thus proving the absence of the determiner from their germ plasm), the offspring are never affected. These conclusions are based on the remarkable pedigree compiled by Nettleship, involving over two thousand individuals. 
Many peculiarities of the skeleton are clearly due to a positive determiner that inhibits the normal development. Thus the case is cited of a father with a deformed clavicle or collar bone; of his seven children, five have the clavicles of a more or less abnormal form. Likewise in polydactylism, or extra-fingeredness, there is some positive factor that induces the formation of the extra toe; but normaltoed persons of a polydactyl strain, being without the determiner, will have all children with five toes only.

The same is true of brachydactyly. There is something that stops the growing of the fingers to the normal length,

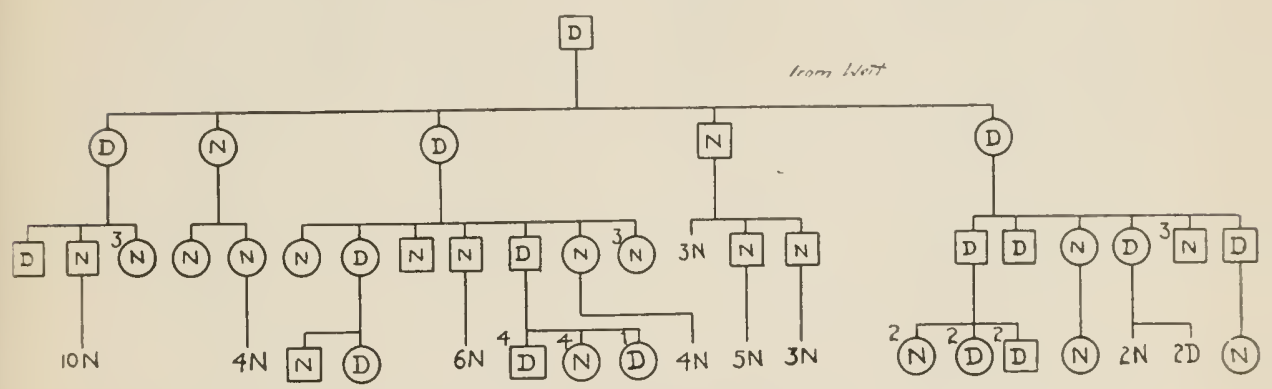

FIG. 85.-Pedigree of a family with diabetes insipidus. D, affected persons

so that if the determiner gets into the zygote from either side of the house, the child will be short-fingered, but not otherwise.

Diabetes is a common disease which seems to belong to this category (Fig. 85). Here again two normal parents may have defective children but only when the defect occurs in the germ plasm of both sides of the house.

The applications of these facts regarding abnormalities and diseases that are of a positive sort have an importance for eugenics. They are all characterized by this, that they usually appear in each generation and do not skip genera- 
tions. They are relatively common in any given family, since half or all of an affected fraternity commonly show the trait. If the trait is an undesirable one and it must not be reproduced, then the eugenical advice is for an affected person to abstain from having children. But an unaffected person belonging to this strain may marry an unaffected person with impunity, and it is immaterial for the inheritance of this trait whether they be cousins or not.

Ve have next to consider the class of abnormalities and weaknesses that are due to the absence of a determiner, to the disappearance of a trait that tends to normality. A good example of such a defect is seen in albinism, already referred to (Fig. 86). In this large pedigree the number of affected individuals is small, and they are frequently derived from two pigmented persons; cousin marriages are common. The defect may be carried in the germ cells of two normal parents; hence its appearance from such parents.

Another example is seen in Thomsen's disease, a disease that is characterized by a slow initial contraction of a muscle after stimulus (Fig. 86). Here again two normal consorts have some affected children and cousin marriages are common.

There is reason for asserting that weakness of the mucous membranes is due to a similar defect (Fig. 88). If both parents are without the determiner for resistance, then of course all offspring will be non-resistant. When one parent is liable to colds and pneumonia and the other has catarrh, the children suffer from tonsilitis, diphtheria, and inflammations of the throat and lungs. But if one parent be non-resistant, and the other, though resistant, have some non-resistant germ cells, at least half of the offspring will be non-resistant. 


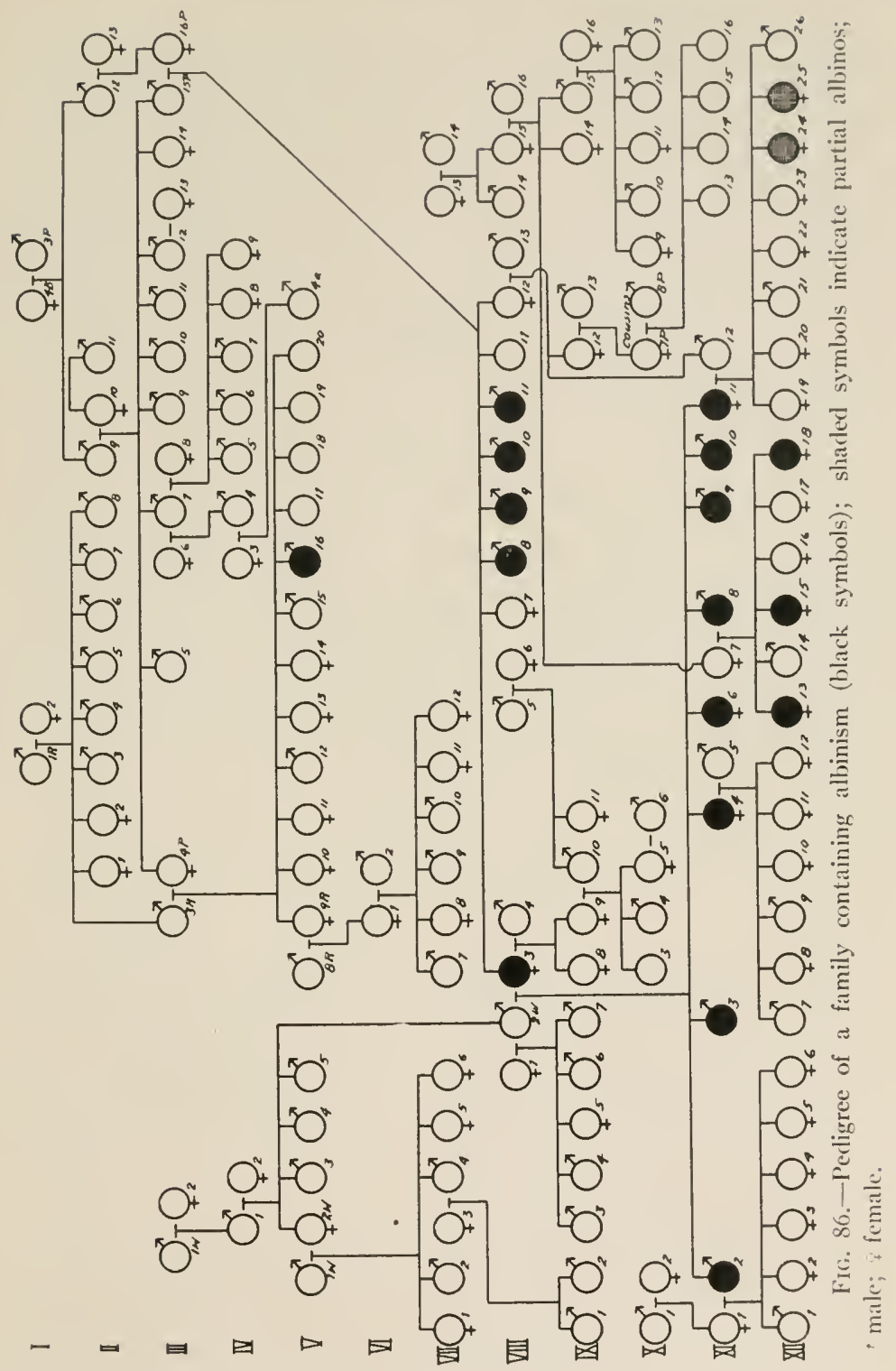


Some eye diseases are clearly due to a defect in the determiner. Such is the case with an inflammatory condition of the retina in which pigment is deposited and the

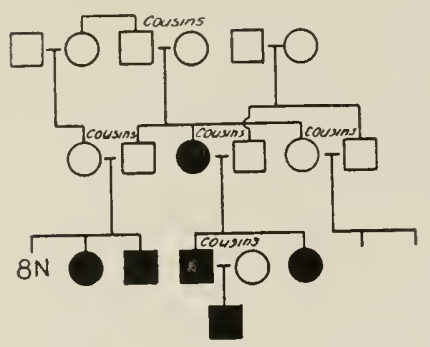

FIG. 87.-Pedigree of Thomsen's disease (black symbols). Appears in cousin marriages even from affected parents; hence due to a defect. Squares indicate males; circles, females.-BERNHARDT. patient ultimately loses his sight (Fig. 89). This disease frequently appears in the children of two normal persons who are cousins, and consequently both carry the defect in their germ cells.

Deaf-mutism also is due to a defect; but the nature of the defect is different in different cases. Deaf-mutism is so varied that frequently two unrelated deaf mutes may have hearing children (Fig. 90). But if the deaf-mute parents be cousins, the chances that the deafness is due to the same unit defect are increased and all of the children will probably be deaf.

We come now to consider mental peculiarities, and here at once enter a vast field in which surprising discoveries have been made in recent years, and which point to the cause of

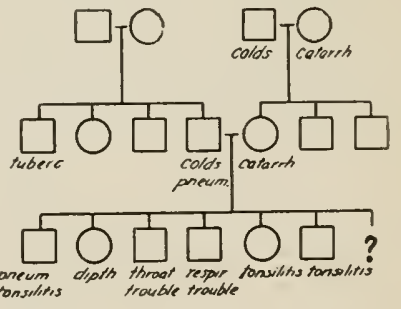

FIG. 88.-Pedigree showing inheritance of a tendency toward colds, catarrh, and respiratory diseases. many of our social difficulties and the way out.

First, consider the facts of feeble-mindedness. This term is a lumber-room and comprises various mental deficiences, such as inability to count, to repeat phrases, to 
learn to write or to draw, to meet difficult situations by intelligent adjustment, to control the appetites and passions, to appreciate moral ideas. Many persons who are not regarded as feeble-minded have some of these or similar defects; the typically feeble-minded are defective in several or many such mental

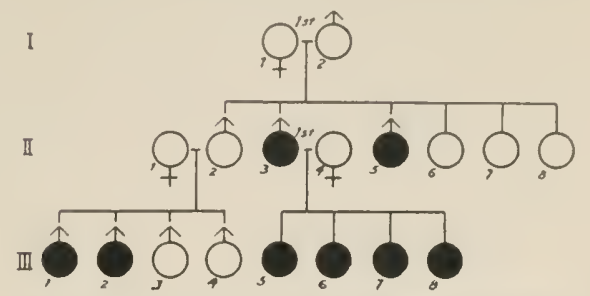

FIG. 89.-Pedigree of retinilis pigmenlosa (black symbols) in a family described by Mooren.-Netrleship. traits. In what follows I shall use feeble-mindedness in the latter sense.

From the studies of Dr. Goddard and others, it appears that when both parents are feeble-minded all of the children will be so likewise; this conclusion has been tested again and again (Fig. 9I). But if one of the parents be normal and of

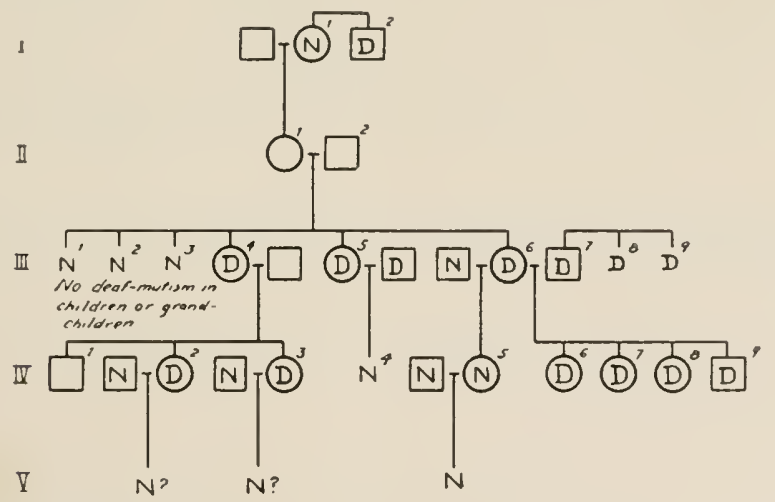

FIG. 90.-Pedigree of a family with deaf mutes (1)) in a large proportion of the later generations.

normal ancestry, all of the children may be normal (Fig. 92); whereas, if the normal person have defective germ 
cells, half of his progeny by a feeble-minded woman will be defective.

Epilepsy and feeble-mindedness may replace each other (as equivalents) in pedigrees. This is well illustrated by

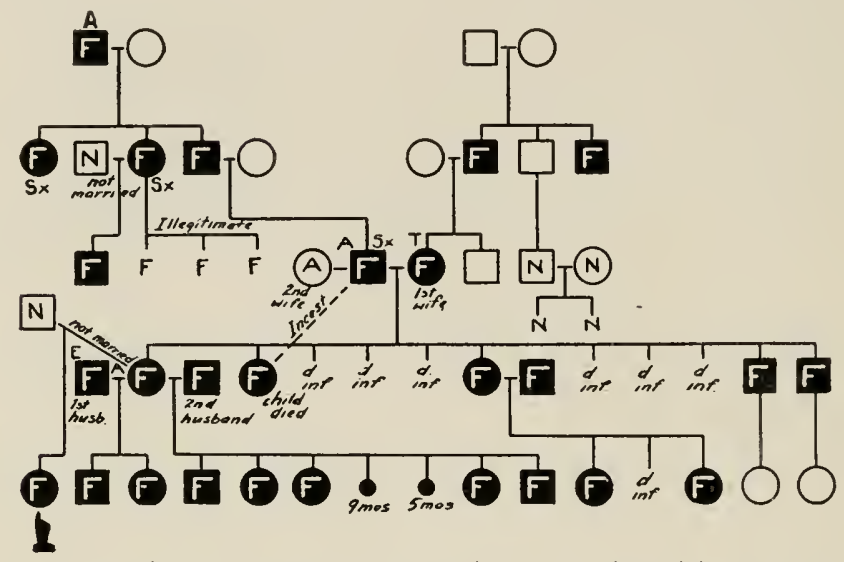

FIG. 9r. - Pedigree of a family with a high proportion of feeble-minded persons (F). Squares, males; circles, females; $d$. inf, died in infancy:-GODDARD.

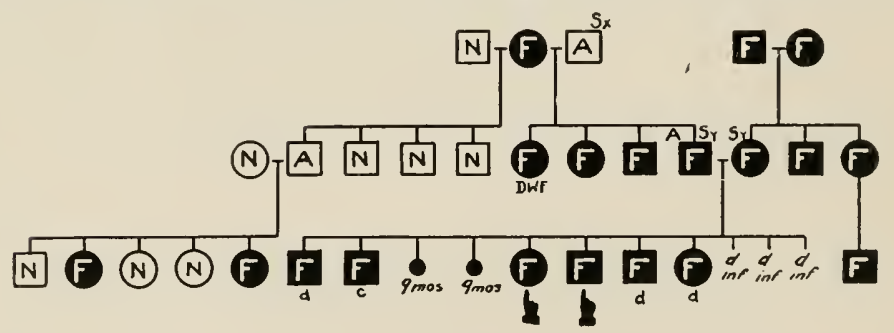

FIG. 92.-Pedigree of a family in which the feeble-minded grandmother married twice; by a normal husband she had normal children; but by an alcoholic, sex-offending $(\mathrm{Sx})$, doubtless feeble-minded husband she had only feebleminded children.-GODDARD.

the figure (Fig. 93) in which a feeble-minded sex offender has by an epileptic daughter two feeble-minded children and one epileptic child.

Many criminals, especially those who offend against the person, are feeble-minded, as is shown by the way they 
occur in fraternities with feeble-mindedness, or have feebleminded parents (Fig. 94). The test of the mental condition of relatives is one that may well be applied by judges in deciding upon the responsibility of an aggressor. It is to be hoped that the conservatism of the law upon this matter may be speedily overcome.

Not only the condition of imperfect mental development, but also that of inability to withstand stress upon the nervous system, may be inherited. From the studies of Dr. Rosanoff and his collaborators, it appears that if both parents be subject to manic depressive insanity or to dementia precox, all children will be neuropathic also (Fig. 95); that if

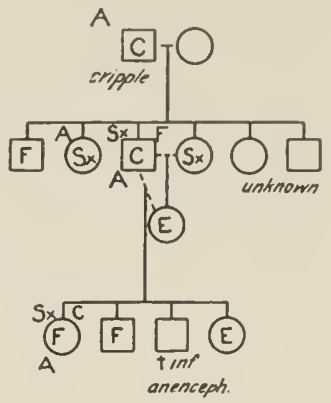

FIG. 93.-The pedigree of a family in whose second generation incest produces an epileptic daughter, but by whose own father she has one epileptic and two feeble-minded children; $\mathrm{A}$, alcoholic; C, criminalistic; E, epileptic; F, feebleminded; Sx, licentious. one parent be affected and come from a weak strain, half

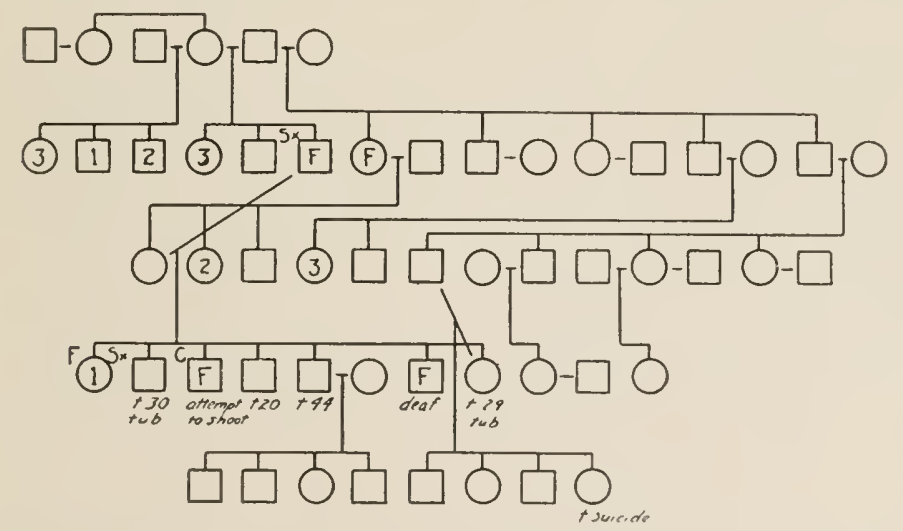

FIG. 94. -Pedigree of a feeble-minded family in which criminalistic (C) and licentious $(\mathrm{Sx})$ traits also appear. 
of the children are liable to go insane; and that nervous breakdowns of these types never occur if both parents be of sound stock.

Even the condition of general nervousness is an indication of a nervous weakness that is, apparently, due to the absence of a determiner. Thus when a person belonging to a neurotic strain marries a normal person whose father died of apoplexy, some neurotic and feeble-minded children may appear in the offspring.

Finally, a study of families with special abilities reveals a method of inheritance quite like that of nervous defect.

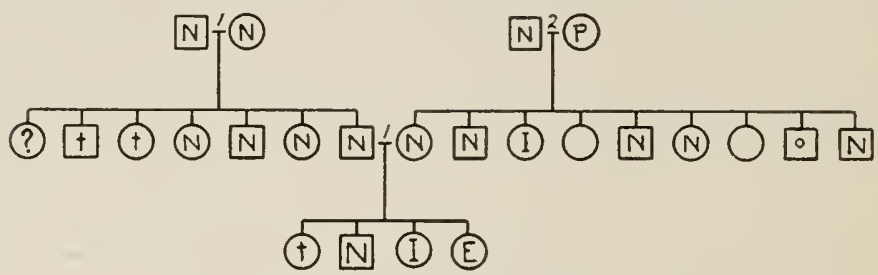

FIG. 95.-Pedigree of a family in which the father's parents (upper left) are both nervous $(\mathrm{N})$ and have four nervous children. The mother is nervous; so were her father and four of her brothers and sisters, while one is insane. Of the three grandchildren one is insane (I), one epileptic (E), and one extremely nervous $(\mathrm{N})$.CANTON AND RosañofF.

If both parents be color artists, or have a high grade of vocal ability or are littérateurs of high grade, then all of their children tend to be of high grade also. If one parent has high ability, while the other has low ability but has ancestry with high ability, part of the children will have high ability and part low. It seems like an extraordinary conclusion that high ability is inherited as though due to the absence of a determiner in the same way as feeblemindedness and insanity are inherited. We are reminded of the poet: "Great wits to madness sure are near allied." Evidence for the relationship is given by pedigrees of men 
Criminality in its worst forms is similarly due to a lack of appreciation of or receptivity to moral ideas.

If we seek to know what is the origin of these defects, we must admit that it is very ancient. They are probably derived from our apelike ancestors in which they were normal traits. There occurs in man a strain that has not yet acquired those traits of inhibition that characterized the more highly developed civilized persons. The evidence for this is that, as far back as we go, we still trace back the black thread of defective heredity.

We have now to answer the question as to the eugenical application of the laws of inheritance of defects. First, it may be pointed out that traits due to the absence of a determiner are characterized by their usual sparseness in the pedigree, especially when the parents are normal; by the fact that they frequently appear where cousin marriages abound, because cousins tend to carry the same defects in their germ plasm though normal themselves; by the fact that two affected parents have exclusively normal children, while two normal parents who belong to the same strain, or who both belong to strains containing the same defect, have some (about 25 per cent) defective children. But a defective married to a pure normal will have no defective offspring.

The clear eugenical rule is then this: Let abnormals marry normals without trace of the defect, and let their normal offspring marry in turn into strong strains; thus the defect may never appear again. Normals from the defective strain may marry normals of normal ancestry; but must particularly avoid consanguineous marriages.

The sociological conclusion is: Prevent the feebleminded, drunkards, paupers, sex-offenders, and criminalistic from marrying their like or cousins or any person belonging 
to a neuropathic strain. Practically it might be well to segregate such persons during the reproductive period for one generation. Then the crop of defectives will be reduced to practically nothing.

I cannot close without referring to a remarkable method of inheritance of human traits, namely, the sex limited. As everyone knows, there are certain traits, such as facial hair, which are associated with one sex; and a tendency to heavy growth of beard may be transmitted by a mother's germ cells to her son. In this case the determiner for heavy beard does not develop in the female, but only in the male, under the stimulus, as it were, of the testicular secretions; or perhaps in the absence of an inhibiting enzyme secreted by the ovary.

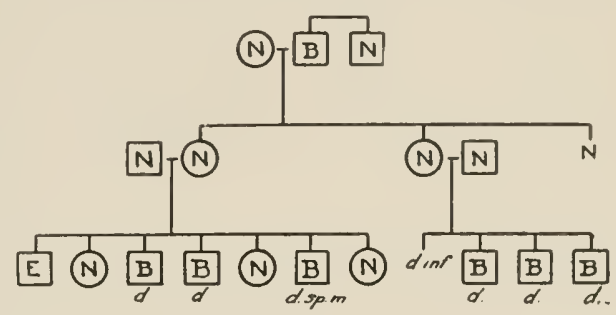

FIG. 97.-Pedigree of family with colorblindness (B).

But in another class of cases the inheritance is most complex. Thus usually only males are color-blind, but they do not transmit their condition to their sons. On the other hand, the normal women of this strain will have color-blind sons (Fig. 97).

This has been a great mystery, but thanks to the recent studies in sex chromosomes by Wilson, Morgan, and others, it is a mystery no longer. It is explained by one fact and one hypothesis. The fact is that the male has only one sex chromosome, while the female has two. The hypothesis is that a factor for distinguishing colors is lacking in the affected male and is lost out of the single sex chromosome of such a male. Now the consequence of these two principles can be seen easily. Let the striated disk ( $S$, Fig. $9 S$ ) 
stand for the single male sex chromosome, which, by hypothesis, lacks the color distinguishing factor. Let the white disk $(W)$ symbolize the absence of a chromosome in a germ cell. Let the black disks $(B)$ represent the two female sex chromosomes with the factor of color-sight. Then the union of $S+B$ gives the female sex and has the determiner for color, albeit simplex. The union $W+B$ gives the male sex and also has the determiner for color-sight. Hence

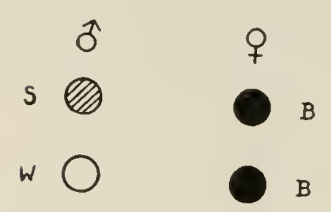

FIG. 98.-Diagram illustrating method of inheriting sex-limited characters. The circles represent sex chromosomes. neither sons nor daughters of a colorblind man are color-blind. If the son marry a normal woman, it is clear that (since no $S$ comes into the union) the children are normal. But if the daughters marry, half of the males will receive the single $S$ chromosome and such will be color-blind. Thus the long famous knight's move form of heredity of color-blindness is explained. Several other traits are inherited in the same way: bleeding, imperfect development of the iris, and atrophy of the optic nerve. In all these cases unaffected males may marry with impunity; but females of the strain who have affected brothers should not have children.

The foregoing considerations bring clearly to mind the great advance that has been made in recent years in the analysis of the inheritance of traits. At last it is possible to give definite advice to those about to marry, or who do not wish to transmit their undesirable traits. Of the method of inheritance of many traits we are still in ignorance. In the absence of detailed knowledge, the best general advice that can be given is this; marry dissimilars. Weakness in any trait should marry strength in that trait; and strength may marry weakness. 


\section{CHAP'TER IX}

THE GEOGRAPHI OF MAN IN RELATION TO EUGENICS

RELATIONS OF BARRIERS TO HUMAN BREEDING

In the period before our Civil War, while men were looking for an excuse if not a justification for slavery the subject of the unity of man's species was much discussed. In these later days, removed from the passions of politics, as a purely academic question the inquiry has been reopened. Ideas have changed much in the intervening fifty or sixty years and now we have to define all over again what is meant by species or race.

In connection with the new ideas of heredity we have gained a new conception of species and race. We now apply the terms indifferently and say a species, or race, is an intergenerating group of individuals distinguished by the possession of one or more unit-characters. As we look over mankind we note at once the groups that have always been distinguished: the Negroes with black skin and woolly hair; the oriental race with olive or yellow skin and (typically) narrow eyes; the American Indian with brown-red skin and long straight hair, and the Caucasians with white skin and high cephalic index. This naïve classification may have sufficed for the dawn of anthropology, but today we recognize its insufficiency. In the group of Caucasians are hundreds of distinctive characters upon each of which a race might be founded. There are the brunette skin and

'Much of the present chapter is reprinted from the author's book. IIcredily' in Relation to Eugenics, and it appears here through the courtesy of the publishers of that book, Messrs. Henry Holt \& Co. 
the blonde; the straight hair and the curly; the flaxen hair and the brown and the red; the blue eyes and the dark; the straight nose, the aquiline, and the pug; the broad head and the high and the narrow; the thin lips and the thick, and so through the categories. The only reason why we do not have distinct species of men distinguished by such traits is because of the extensive hybridization that man is undergoing. Everywhere, brown eyes mate with blue, black hair with flaxen, curly with straight, and so on. Man's potential races are not realized just because of the universal interfertility of the different races and because of the mobility of man's habitat.

Now is there any evidence aside from a-priori considerations for testing this view? Are the potentialities that we assume anywhere realized? Is the theory of man's universal hybridization more than a figment of the imagination?

First, let us admit that evidence for the unity of, say, the Caucasian race has been offered by the biometricians. They have said if the race is homogeneous it will show itself by the biometric test. Measure a trait in Io,000 individuals; and plot the relative frequency of the different values found. If that frequency rises in a gentle curve from its lowest value to a maximum at some middle value and then falls again smoothly to the highest value the curve is a simple curve and this has been regarded as proving a unitpopulation. But it does not prove it; for we now realize that even the apparently simple curve may be the resultant of many more elementary curves whose number diminishes uniformly on both sides of the center. The elementary curves are the ones that include the fluctuations of the real units. 
Second, the real units may be isolated by the simple process of preventing the random hybridization and ensuing breeding within the type. This result has been nearly realized in small oceanic islands, and in other isolated communities. It will be interesting to look at some of these isolated places and learn what has been produced in them.

At Swans' Island, Maine, much consanguinity in marriage occurs; cousin marriages are the rule. A consequence has been that the defect of feeble-mindedness is unusually common and, were the process to continue for many more generations, a race with this trait, among others, would doubtless become established.

At Western Martha's Vineyard a careful genealogical study has been made by Dr. Alexander Graham Bell and much consanguineous marriage has been found. Here is, or was, being formed a deaf-mute colony; one out of every twenty-five was already a deaf mute.

At Block Island, with a population of fifteen hundred, much consanguineous marriage has occurred, and a nonfecund strain has been isolated. On the Banks off Palmico Sound consanguineous marriage occurs with extraordinary frequency; and a strain characterized by suspicion, insanity, and mental dulness is being formed. At George Island near Eleuthera Island, one of the Bahamas, long inbreeding has produced a race that tends toward dwarf stature and eye defects, including cataracts.

What is true of islands holds for other isolated situations. A physician at an extreme point of the peninsula of Dorchester County, Maryland, writes that marriages there are usually consanguineous and a race of dwarfs and cripples is being formed. Mountain valleys of the Ramapo, Cats- 
kill, Taconic, and Adirondack masses show many endogamous centers. In one place a race of criminals is being formed; in another a feeble-minded strain; in another an albino race, and so on. There is reason for thinking that the valleys of eastern Kentucky and Tennessee are centers of inbreeding and nearly pure races are being formed there. In larger settled countries the process has gone farther. From the Chin Hills, Burmah, one hears: "Rau Vau Village has been isolated for about seven generations. It contains about sixty houses and possibly two hundred inhabitants. Of these ten are idiots, many are dwarfs, and some hydrocephalic. A number of cases of syndactylism or webbing of hands, and brachydactyly occur."

Only slightly less important than the geographical barriers are the social. A public institution brings together men and women so intimately that marriages frequently occur after leaving the institution. Thus two persons with the same trait become parents. Almshouses in which segregation of the sexes is imperfect yield numerous depauperate and imbecile offspring, and there is reason for suspecting that sanatoria and some hospitals for the "curable" insane lead to marriage of two weak persons. That institutions for the deaf lead to the marriage of similarly defective is notorious. Thus Dr. Bell, who has long warned us of the imminent danger of the formation of a deaf variety of the human race in America, says: "I desire to direct attention to the fact that, in this country deaf mutes marry deaf mutes. An examination of the records of some of our institutions for the deaf and dumb reveals the fact that such marriages are not the exception but the rule."

The barrier of language is extremely important in promoting consanguineous marriages, or the matings of persons 
with the same defect. Thus with regard to deaf mutes, Bell says: "The practice of the sign language hinders the acquisition of the English language; it makes deaf mutes associate together in adult life, and aroid the society of hearing people; it thus causes the intermarriage of deaf mutes and the propagation of their physical defect." The importance of this barrier is seen among recent immigrants. These tend to herd together, largely because of a desire to be with people who speak the same language. Thus immigration, instead of directly tending to promote matings of dissimilar and unrelated blood, has, at first, an exactly opposite effect.

The barrier of race is of the very greatest importance in promoting marriages of kin-especially if one race be in a marked minority, as the Negroes are in New Hampshire and the whites are in the Mississippi River bottom, around Vicksburg, or in parts of the West Indies.

Finally the barrier of religious sect has been erected again and again to insure the intermarriage of the faithful only. This is illustrated by the teachings of the Society of Friends and smaller sects, such as the Dunkers, Shakers, and Amish. Of the Dunkers it is written: "In their early history marriage out of the church was punishable by expulsion. ${ }^{x}$ It is still frowned upon but the process of liberalization now in progress had modified the attitude of the church. In some congregations families intermarry generation after generation. But the degree of kinship is not so close that any evil results appear in the offspring." Nevertheless one sees the danger that any small sect with such tenets runs. A critical study of the Amish of Pennsylvania with much marriage of kin shows a sufficient progeny of epilepsy and crippled children to serve as a

${ }^{1}$ Chronicon Ephratense, 96, 246. 
warning that a defect is in the blood of some of the strains that in time will affect the entire sect who remain in that part of the country.

\section{MIGRATIONS AND THEIR EUGENIC SIGNIFICANCE}

The human species has come to occupy the entire habitable globe. This fact is mute testimony of man's migratory capacity and tendencies. Just as the Norwegian lemming has been observed, in consequence of several years of favorable conditions for breeding in its mountain home, to spread over the surrounding territory in great bands, seeking less crowded breeding grounds; even as the army worm and the grasshopper swarm from their native territory; so man, also, under the pressure of crowded conditions, poverty, and oppression, or lured by brighter prospects elsewhere, may move in hordes to other lands that seem to offer better opportunities. Thus Asia seems to have debouched her surplus population upon Europe in the shape of the Huns during the fourth and fifth centuries of our era and the Turks during the fourteenth and fifteenth centuries. So the Anglo-Saxons and the Normans successively swarmed upon England. So, among savages, the Masai of Africa moved upon the neighboring tribes and established themselves over much of southeastern Africa. So in the last three centuries the Americas and Australia have witnessed the greatest migrations that the world has ever seen, hundreds of thousands annually coming from overcrowded Europe, and Asia to the "New World."

For us in America the phenomena of migration should have a special interest. Excepting for the few scores of thousands of Indians there was a continent devoid of a population - a clean slate upon which history was to be 
written and where the effect of "blood" in determining that history might be traced.

Since the first few scores of thousands of immigrants had the greatest influence on the ideals of the colonies they established and since their blood has had the longer time to show its effect, and since their traits have had the greatest chance to disseminate widely, they deserve special consideration.

On the James River the first settlers consisted chiefly of "discredited idlers and would-be adventurers," more than half of them "gentlemen" of good family but untrained in labor, trusting for a change of fortune in the new land. Even later, men, women, and children were sent by the London Company to colonize the new land and that company was not particular as to quality. Even felons, murderers, and women of the streets were at times sent over from London to relieve the city of them, and the governor, who was a pure euthenist and seemed to think the better environment would cure their evil ways, welcomed all.

But a better blood soon crowded into Virginia to redeem the colony. Upon the execution of Charles I (I649) a host of royalist refugees sought an asylum here, and the immigration of this class continued even after the Restoration. By this means the province was enriched by a germ plasm which easily developed such traits as good manners, high culture, and the ability to lead in all social affairs-traits combined in remarkable degree in the "first families of Virginia." From this complex and the similar complex of Maryland has come much of the bad blood that found the retreats of the mountain valleys toward Kentucky and Tennessee to its liking and that spread later into Indiana and Illinois and gave rise, in all probability, to the Ishmael- 
ites, a family of which hundreds have been supported in the almshouses and jails of Indiana. From this complex came also some of America's greatest statesmen and warriors, the Randolphs, the Marshalls, the Madisons, the Curtises, the Lees, the Fitzhughs, the Washingtons, and many others born with the instinct to command. Such are the descendants of the high-spirited cavaliers. It might have been predicted that the future state would be "the Mother of Presidents," and that in a civil war the severest battles should be fought on her soil.

Farther north, at Manhattan Island, a settlement was being made by another sort of people: a band of Dutch traders. The fur trade with the Indians waxed profitable. The more venturesome established trading-posts up the North River, even as far as the present site of Albany; others went east as far as the Connecticut River. They maintained friendly relations with the Indians, as the main source of their wealth, and under their protection established trading-posts, even along the valley of the Mohawk. Little wonder that such blood, under the favorable environment of an admirable location, has created the commercial center of the western world.

On the bleak coasts of New England were being founded settlements of idealists, men who were willing to undergo exile for conscience' sake. They included many scholars like the pastor Robinson; Brewster who, while self-exiled at Leyden, instructed students at the University; John Winthrop, "of gentle breeding and education"; John Davenport, of New Haven, whom the Indians named "heap study-man." Little wonder that the germ plasm of these colonies of men of deep convictions and scholarship should show its traits in the great network of its 
descendants and establish New England's reputation for conscientiousness and love of learning and culture. As it was almost the first business of the founders of the colonies of Massachusetts Bay and New Haven to found a college, so their descendants - the families of Edwards, Whitney, Dwight, Eliot, Lowell, Woolsey, and the rest-have not only led in literature, philosophy, and science, but have carried the lamps of learning across the continent, lighting educational beacons from Boston to San Francisco. Nor is it an accident that on the soil tilled by these dissenters from the Established Church of England should be spilled the first blood of the American Revolution.

Later, to the shores of the Delaware, Penn led his band of followers, consisting of men and women whose natures were attracted to his principles of thrift, absence of show, and non-resistance. The germ plasm of his followers soon peopled Penn's woods, and it is not due solely to chance that Pennsylvania has the largest number of homes owned by their occupants and free from debt of any state.

Thus the characteristics of each commonwealth were early determined by the traits of the persons who were attracted toward them. These traits still persist in their dwindling descendants who strive to secure the preservation in the state of the ideals inculcated by their forefathers.

One common characteristic these early immigrants had, which led them to leave family and friends, to undergo the trials of the long sea voyage in small ships and to settle in a rigorous climate among unreliable savages, and that was a willingness to break with tradition, to exchange the old for the new and better. This trait, that amounts in extreme cases to a wanderlust, is illustrated by the history of many a pioneer. For example, Simeon Hoyt landed in Salem, 
Mass., in 1628 ; went in the first company of settlers to Charleston (I629); went to Dorchester (I630) with the first company of settlers there; joined the church at Scituate (I635), and built a house there; then, probably in the spring of 1636 , migrated to Windsor, Connecticut Colony, which he helped found. In 1649 he was granted land at Fairfield, and in 1657 he died at Stamford. Thus in the space of thirty years Simeon Hoyt lived in seven villages in America and was a founder of at least three of thema truly restless spirit, like many another settler, and the parent of a restless progeny!

Still another example is that of Hans Jorst Heydt of Strasburg. He fled to Holland when his native town was seized by Louis XIV, married there Anna Maria DuBois, a French Huguenot refugee from Wicres, and came with her to America and settled at New Paltz on the Hudson about I 7 IO. Schismatic dissensions having broken out in the new colony, Heydt, with others, left and settled, about I7 I7, in Philadelphia County, not far from Germantown, where he acquired several hundred acres of land, established a colony, built mills, and entered upon various commercial enterprises of magnitude. In I73 I, having acquired a grant of forty thousand acres of land in the Shenandoah Valley, he migrated thither, became known as Baron Hite, and died there in 1760 . One of his friends, Van Metre, who originally settled at New Paltz, had moved first to Somerset County, New Jersey, then to Salem County in the same colony; later to Prince George's County, Maryland, and, finally, to Orange County, Virginia. These are examples, merely, of the restlessness-often enterprising restlessness - of the early settlers, and it persists in their descendants. 
Now all of these migrations have a profound eugenic significance. The most active, ambitious, and courageous blood migrates. It migrated to America and has made her what she has become; in America another selection took place in the western migrations, and what this best blood - this crême de la crême- did in the West all the world knows. Great cities like Chicago, with its motto "I will," arose in a generation or two to the front rank of world metropolises, and New England, the early home of the sewing machine and the cotton gin, has yielded the palm to the Central West, the home of the reaping machine and the aeroplane.

And when the best and strongest migrated the weaker minds were left behind to breed in the old homestead. A recent report of the British "Committee on Physical Deterioration" contains the testimony of Dr. C. R. Browne about conditions in the west of Ireland. He says: "The sound and the healthy-the young men and young women from the rural districts emigrate to America in tremendous numbers, and it is only the more enterprising and the more active that go, as a rule." And Dr. Kelly, the Roman Catholic bishop of Ross testified: "For a considerable number of years it has been only the strong and vigorous that gothe old people and the weaklings remain behind in Treland." And even in New England we see signs of decadence of the old stock and men speak of racial deterioration. But the race as a whole has not deteriorated but only the New England representatives - the left-behinds of the grand old families, whose stronger members went west.

Likewise in the rural and semi-rural population within a hundred miles of our great cities, we find a disproportion of the indolent, the alcoholic, the feeble-minded, the ne'er- 
do-well. Thus our great cities lure to themselves the best of the rural protoplasm and surround it with conditions that discourage reproduction, either by creating a disinclination to marriage or making it inconvenient and expensive to have children. So our great cities act anti-eugenically, sterilizing the best and leaving the worst to reproduce their like.

THE INFLUENCE OF THE SINGLE GERM PLASM ON THE RACE

As one stands at Ellis Island and sees pass the stream of persons, sometimes five thousand in a day, who go through that portal to enter the United States and, for the most part, to become incorporated into it, one is apt to lose sight of the potential importance to this nation of the individual, or, more strictly, the germ plasm that he or she carries. Yet the study of extensive pedigrees warns us of the fact. Every one of those peasants will, if fecund, play a rôle for better or worse in the future history of this nation. Formerly, when we believed that traits blend, a characteristic in the germ plasm of a single individual among thousands seemed not worth considering -it would soon be lost in the melting-pot. But now we know that unit-characters do not blend; that after a score of generations the given characteristic may still appear unaffected by the repeated union with foreign germ plasm. So the individual, as the bearer of a potentially immortal germ plasm with immutable traits, becomes of the greatest interest. A few examples will illustrate this law and its practical importance.

Elizabeth Tuttle.-From two English parents, sire at least remotely descended from royalty, was born Elizabeth Tuttle. She developed into a woman of great beauty, of tall and commanding appearance, striking carriage, "of 
strong will, extreme intellectual vigor. On November I9, I667, she married Richard Edwards of Hartford, Connecticut, a lawyer of high repute and great erudition. Like his wife he was very tall, and as they both walked the Hartford streets their appearance invited the eyes and admiration of all." In I69I, Mr. Edwards was divorced from his wife. After his divorce Mr. Edwards remarried and had five sons and a daughter by Mary Talcott, a mediocre woman, average in talent and character and ordinary in appearance. None of Mary Talcott's progeny rose above mediocrity and the descendants gained no abiding reputation.

Of Elizabeth Tuttle and Richard Edwards the only son was Timothy Edwards, who graduated from Harvard College in I69I, gaining simultaneously and highly exceptionally the two degrees of Bachelor of Arts and Master of Arts. He was pastor of the church in East Windsor, Connecticut, for fifty-nine years. Of his eleven children the only son was Jonathan Edwards, one of the world's great intellects, pre-eminent as a divine and theologian, president of Princeton College. Of the descendants of Jonathan Edwards much has been written; a brief catalogue must suffice: Jonathan Edwards, Jr., president of Union College; Timothy Dwight, president of Yale; Sereno Edwards Dwight, president of Hamilton College; Theodore Dwight Woolsey, for twenty-five years president of Yale College; Jared Sparks, president of Harvard College, IS49-53; Sarah, wife of Tapping Reeve, founder of Litchfield Law School, herself no mean lawyer; Daniel Tyler, a general of the Civil War and founder of the iron industries of northern Alabama; Ann Maria, wife of Edwards Amasa Park, president of Andover Theological Seminary, herself as 
astute a thinker as her clerical spouse; Timothy Dwight the second, president of Yale University from I886 to I8g8; Theodore William Dwight, founder and for thirty-three years warden of Columbia Law School; Henrietta Frances, wife of Eli Whitney, inventor of the cotton gin, who, burning the midnight oil by the side of her ingenious husband, helped him to his enduring fame; Merrill Edward Gates, president of Amherst College; Catherine Maria Sedgwick, of graceful pen; Charles Sedgwick Minot, authority on biology and embryology in the Harvard Medical School; Edith Kermit Carow, wife of Theodore Roosevelt, and Winston Churchill, the author of Coniston. These constitute a glorious galaxy of America's great educators, students, and moral leaders of the Republic.

The remarkable qualities of Elizabeth Tuttle were in the germ plasm of her four daughters also: Abigail Stoughton, Elizabeth Deming, Ann Richardson, and Mabel Bigelow. All of these have had distinguished descendants of which only a few can be mentioned here. Robert Treat Paine, signer of the Declaration of Independence, was descended from Abigail; the Fairbanks Brothers, manufacturers of scales and hardware at St. Johnsbury, Vermont, and the Marchioness of Donegal were descended from Elizabeth Deming; from Mabel Bigelow came Morrison Waite, chief justice of the United States, and the law author, Melville M. Bigelow; from Ann Richardson proceeded Marvin Richardson Vincent, professor of Sacred Literature at Columbia University and the Marchioness of Apesteguia, of Cuba. Thus, numerous scholars, inventors, and publicists trace back their origin to the germ plasm from which (in part) Elizabeth Tuttle also was derived, but of which, it must never be forgotten, she was not the author. 
The first families of Virginia.-This remarkable galaxy arose by the intermarriage of representatives of various English aristocratic families. The story of these early matings is briefly as follows: Richard Lee, of a Shropshire family that held much land, and many of whose members had been knighted, went, during the reign of Charles I, to the colony of Virginia as secretary and one of the king's Privy Council. "He was a man of good stature, comely visage, enterprising genius, sound head, vigorous spirit, and generous nature." He gained large grants of land in Virginia. His son, Richard, married in 1674 , Lactitia, daughter of Henry Corbin and Alice Eltonhead. The Corbins were wealthy and extensive landowners in England for fourteen generations, and the Eltonheads were also an aristocratic family and extensive landowners of Virginia, holding high offices in the colony.

Richard and Laetitia Lee had six sons and one daughter, Ann. Ann married Col. William Fitzhugh, a descendant of the English barons, military men, and parliamentarians of that name. Their eldest son married a Carter, and one of their granddaughters and one of their sons married a Randolph; their daughter Mary, married George Washington Parke Custis, and became the grandmother of Robert E. Lee. Richard Lee, Jr., had children who married into the families of Fairfax and Turberville. A brother of Richard, Thomas, was president of the council and at one time acting governor of the colony. He married Hannah Ludwell, descendant of a brother of the statesman, Lord Cottington; one of their sons, Richard Henry Lee, prepared at the Continental Congress the resolutions for independence: another, Francis Lightfoot Lee, was a member of Congress and still another, Thomas, a judge of the General Court. 
Another son of Richard and Laetitia Lee was Henry, who married Mary Bland, a descendant of Sir Thomas Bland, and a granddaughter of Theodorick Bland, speaker of the House of Burgesses and member of the Council. Their three sons were all members of the House of Burgesses and some were in the House of Delegates, in conventions and in the state senate. Such was the product of the first families of Virginia - statesmen, military menthe necessary product of their germ plasm.

The Kentucky aristocracy.-Nearly two centuries ago, John Preston of Londonderry, Irish born though English bred, married the Irish girl, Elizabeth Patton, of Donegal, and to the wilderness of Virginia took his wife and built their home, Spring Hill.

Of this union there were five children: Letitia, who married Colonel Robert Breckiniridge; Margaret, who married Rev. John Brown; William, whose wife was Susannah Smith; Anne, who married Colonel John Smith; and Mary, who married Benjamin Howard. ... . From them have come the most conspicuous of those who bear the name of Preston, Brown, Smith, Carrington, Venable, Payne, Wickliffe, Wooley, Breckinridge, Benton, Porter, and many other names written high in history.

They were generally persons of great talent and thoroughly educated; of large brain and magnificent physique. The men were brave and gallant, the women accomplished and fascinating and incomparably beautiful. There was no aristocracy in America that did not eagerly open its veins for the infusion of this Irish blood; and the families of Washington and Randolph, and Patrick Henry, and Henry Clay, and the Hamptons, Wickliffes, Marshalls, Peytons, Cabells, Crittendens, and Ingersolls felt proud of their alliances with this noble Irish family.

They were governors and senators and members of Congress, and presidents of colleges and eminent divines, and brave generals from Virginia, Kentucky, Louisiana, Missouri, California, Ohio, New York, Indiana, and South Carolina. There were four governors of old 
Virginia. They were members of the cabinets of Jefferson, and Taylor, and Buchanan, and Lincoln. They had major-generals and brigadier-generals by the dozen; members of the Senate and House of Representatives by the score; and gallant officers in the army and nary by the hundred. They furnished three of the recent Democratic candidates for Vice-President of the United States.

The quotation has a scientific value in comparison with the product of Elizabeth Tuttle. The New England family glows with scholars and inventors; the Virginia and Kentucky families with statesmen and military men. The result is not due merely to the difference in the characteristics of Elizabeth Tuttle, Richard and Laetitia Lee, John and Elizabeth Preston respectively, but to the different traits of the New England settlers as a whole, and the Virginia cavalier-colonists as a body. The initial person becomes a great progenitor largely because of some fortunate circumstance of personal gift or excellent reputation that enables his offspring to marry into the "best blood."

The Jukes.-On the other hand, we have the striking cases of families of defectives and criminals that can be traced back to a single ancestor. The case of the "Jukes" is well known. We are first introduced to a man known in literature as "Max," living as a backwoodsman in New York state, and a descendant of the early Dutch settlers; a good-natured, lazy sot, without doubt of defective mentality. He has two sons who marry two of six sisters whose ancestry is uncertain, but of such a nature as to lead to the suspicion that they are not full sisters. One of these sisters is known as "Ada Juke," also as "Margaret, the mother of criminals." She was indolent and a harlot before marriage. Besides an illegitimate son she had four legitimate children. The first, a son, was indolent, licentious, and syphilitic; he married a cousin and had eight children, 
all syphilitic from birth. Of the seven daughters, five were harlots and of the others one was an idiot and one of good reputation. Their descendants show a preponderance of harlotry in the females and much consanguineous marriage. The second son was a farm laborer, was industrious, and saved enough to buy fourteen acres of land. He married a cousin and they produced three still-born children, a harlot, an insane daughter who committed suicide; an industrious son, who, however, was licentious, and a pauper son. The first daughter of "Ada" was an indolent harlot who later married a lazy mulatto and produced nine children, harlots and paupers, who produced in turn a licentious progeny.

Ada had an illegitimate son who was an industrious and honest laborer and married a cousin. Two of the three sons were licentious and criminalistic in tendency, and the third while capable, drank and received outdoor relief. All of the three daughters were harlots or prostitutes, and two married criminals. The third generation shows the eruption of criminality. Excepting the children of the third son, none of whom was criminalistic, we find among the males twelve criminals, one licentious, five paupers, one alcoholic, and one unknown; none was a normal citizen. Among the females, eight were harlots, one a pauper, one a vagrant, and two unknown; none was known to be reputable. Thus it appears that criminality lies in the illegitimate line from Ada, and not at all in the legitimate -doubtless because of a difference in germ plasm of the fathers.

The progeny of the harlot, Bell Juke, is a dreary monotony of harlotry and licentiousness to the fifth generation. Two in the fourth generation there are, and two in the fifth, 
against whom there is nothing and their progeny mostly moved to another neighborhood and are lost sight of. Very likely they have married into stronger strains and are founders of reputable families.

The progeny of Effie Juke and the son of Max (a thief) show to the fifth generation a different aspect. Some larceny and assault there are, and not a little sexual immorality, but pauperism is the prevailing trait.

Thus, in the same environment, the descendants of the illegitimate son of Ada are prevailingly criminal; the progeny of Bell are sexually immoral; and the offspring of Effie are paupers. The difference in the germ plasms determine the difference in the prevailing trait. But however varied the forms of non-social behavior of the progeny of the mother of the Juke girls, the result was calculated to cost the state of New York over a million and a quarter of dollars in seventy-five years-up to 1877 , and their protoplasm has been multiplied and dispersed during the subsequent thirty-four years, and is still going on.

The Ishmaelites.-As another example of a great family tracing back to a single man may be taken "The Tribe of Ishmael" of central Indiana, as worked out, under the direction of Rev. Oscar C. McCulloch, of the Charity Organization Society, Indianapolis. The progenitor of this tribe, Ben Ishmael, was in Kentucky as far back as I790, having come from Maryland through Kentucky. One of the sons, John, married a half-breed woman, and came into Marion County, Indiana, about I840. His three sons who figure in this history married three sisters from a pauper family named Smith. They had altogether fourteen children who survived, sixty grandchildren, and thirty greatgrandchildren, living in ISSS. 
Since 1840 , this family has had a pauper record. They have been in the Almshouse, the House of Refuge, the Woman's Reformatory, the penitentiaries, and have received continuous aid from the townships. They are intermarried with the other members of this group, .... and with over two hundred other families. In this family history are murders, a large number of illegitimacies, and of prostitutes. They are generally diseased. The children die young. They live by petty stealing, begging, and ash-gathering. In summer they "gypsy" or travel in wagons, east or west. We hear of them in Illinois about Decatur and in Ohio about Columbus. In the fall they return. They have been known to live in hollow trees, on the river bottoms or in empty houses. Strangely enough, they are not intemperate to excess.

Ah, that, in the hordes pressing at the gate at Ellis Island, we could distinguish the John Prestons from the Ben Ishmaels of the future!

\section{THE EUGENICS MOVEMENT}

Since the time of Plato there have not been lacking persons who have urged that the human race would be improved were more attention paid to marriage matings. But, in recent years, these ideas have become so widespread and have been urged with such vigor, as to warrant us in speaking of a present eugenics movement. There are two chief impulses, it seems to me, for this modern movement, both world-wide. The first of these is a conviction that there is a great proportional increase in feeble-mindedness in its protein forms - a great spread of animalistic traitsand of insanity. When a state like New York spends oneseventh of its state income for the care of the insane it is not strange that many of its citizens are inquiring why this is and whether there is any end to the increasing proportion of the state's income that must be spent in caring for those who cannot aid themselves. The proportion of those who are feeble-minded in such various directions as to constitute 
the feeble-minded class is estimated at 3 per cent of our population, and were we to include drunkards, paupers, grave sex-offenders, the criminalistic, the insane, and those with innate physical weaknesses that render them for the most part incompetent, it seems a safe estimate that $S$ per cent of our population are far from having the capacities of effective men and women, able, not merely to support themselves, but really to push forward the world's work. The cost of caring for those who cannot care for themselves because of their bad breeding is very heavy-perhaps two hundred million or more a year. A study of the cause of the increase of dependents indicates that it is because the birth rate of the better classes is constantly falling; a Harvard class does not reproduce itself and at the present rate, one thousand graduates of today will have only fifty descendants two hundred years hence. On the other hand, recent immigrants and the less effective descendants of the earlier immigrants still continue to have large families; so that from one thousand Roumanians today in Boston, at the present rate of breeding, will come a hundred thousand two hundred years hence to govern the fifty descendants of Harvard's sons! Such facts as these have awakened the people to a sense of the omnipotence of human breeding.

The other impulse is the spread of knowledge of the modern principles of heredity; and an appreciation of the facts, first, that they afford a clear method in detail for improving the blood of the nation, and, secondly, that the results of this study can be set forth so simply and clearly that they may become a part of our social idealism and may serve to point the way to useful legislation. Heredity will save the people from the perdition that is to come.

The realization of this fact has led to activity in various directions. In Germany, an International Society of Race 
Hygiene has been organized and in England exists a Eugenics Education Society which publishes the Eugenics Review, and is organizing an International Congress of Eugenics for I9I2. The Eugenics Education Society has fostered the formation of several branches in the United Kingdom. For some years Francis Galton maintained a Eugenics Laboratory, directed by Professor Karl Pearson, that has published a valuable Treasury of Human Inheritance, and was lately well endowed at his death.

In America one of the first undertakings in Eugenics was that of Dr. Alexander Graham Bell, who was much impressed by the consequences of marriages of the deaf in America. He founded the Volta Bureau in Washington, which contains extensive records of the deaf.

In IS8I Mr. Loring Moody, of Boston, who was the organizer of the Association for the Prevention of Cruelty to Children and assisted in the foundation of the Association for the Prevention of Cruelty to Animals, organized an Institute of Heredity, but his death soon after brought his plans to naught.

In October, I910, there was started at Cold Spring Harbor on Long Island, the Eugenics Record Office, which seeks to be a clearing-house for data on human blood lines in America. It has collected several hundred records of family traits and made extensive studies into the pedigrees of the feeble-minded, epileptic, paupers, and insane. This office is publishing a Bulletin. In time we shall have there, we expect, data that will be useful to those contemplating marriage. In various directions we hope to play an important part in creating a sentiment and a knowledge that shall lead to the improvement of the blood of the American people. 
INDEX 



\section{INDEX}

Albinism, 278

Alcoholism, 285

Amphibians, 2 I I

Appetency, ro

Ascaris, 45

Bacteria, work with, 202

Bateson, W., work of, 170

Beetles, work with, $18 \mathrm{r}, 2 \mathrm{r} 3,2 \mathrm{r} 6$

Biffen, R. H., work of, 124

Bigelow, Mabel, pedigree of, 302

Bigelow, Melville M., pedigree of, 302

Biometry", 16

Breckinridge, Robert, Col., pedigree of, 304

Brown, John, Rev., pedigree of, 304

Buchanan, R. E., work of, 203

Burbank, Luther, work of, I 30

Capsella, work with, 203

Carman, E. S., work of, 130

Carow, Edith Kermit, pedigree of, 302

Castle, William E., 39, 62; work of, Ir 2, I 49

Characters, coupled and antagonistic, Ior; nulliplex, 27I; positive, negative, duplex, 270

Chickens, work with, 146

Chromatin, 26

Chromosomes, 26, 45, 66, 287

Churchill, Winston, pedigree of, 302

Citrus fruit, work with, 128

Clay, Henry, pedigree of, 304

Correns, C. E., work of, 4 I

Coulter, John MI., 3, 22

Cuénot, work of, 65

Custis, George Washington Parke, pedigree of, 303

Cytoplasm, 24
Daphnia, work with, 2 10

Darwin, Charles, work of, 39, 142, 168

Darwin, Erasmus, 9

Davenport, C. E., 269, 289; work of, 149

Davis, B. M., work of, I 99

Deaf-mutism, 280

Dementia, 283

Deming, Elizabeth, pedigree of, 302

Determiner, 269; absence of, 279

DeVries, Hugo, work of, 4I, I 70

Dihybrids, 93

Dominance, 105

Drosophila, work with, 75,213

Dwight, Sereno Edwards, pedigree of, 3 or

Dwight, Theodore William, pedigree of, 302

Dwight, Timothy, pedigree of, 30 I

East, Edward Murray, 83 , Ir 3

Edwards, Jonathan, pedigree of, $30 \mathrm{I}$

Edwards, Richard, pedigree of, $30 \mathrm{r}$

Edwards, Timothy, pedigree of, $30 \mathrm{I}$

Egg, 3I, 45

Emerson, R. A., work of, Ior

Environment, 9

Epilepsy, 282

Eugenics, 269, 286; geography of man in relation to, 289; organization of movement, 308 ; in relation to migrations, 294

Evolution, conception of, 4 ; explanation of, 7 ; fact of, 5 ; method of, 39 ; Eyes, inherited characters, 270,276

Feeble-mindedness, 280

Fitzhugh, William Col, pedigree of, 303 
Gager, C. S., work of, 205, 208, 222

Galton, F., work of, I6

Gametes, 29, 62, 87 , I4I

Gametophyte, 34

Gates, Merrill Edward, pedigree of, 302

Gates, R. R., work of, 199

Genetics, 83

Genotype, i io

Geography, in relation to eugenics, 289

Germ plasm, I4I ; direct modification of, I65; influence of the single, 300 ; sudden transmutation in, 167

Goethe, work of, 9

Guinea-pigs, work with, 42, I 49

Guthrie, C. G., work of, 146

Hair, characters of, 272

Henry, Patrick, pedigree of, 304

Heredity, I 7, 42, 289; and sex, 62

Heterozygote, 62

Heydt, Hans Jorst, pedigree of, 298

Homozygote, 62

Howard, Benjamin, pedigree of, 304

Hoyt, Simeon, pedigree of, 297

Human breeding, relations of barriers to, 289

Hybridization, I 8

Hybrids, 91, 122 , 166

Inheritance, 42, 83 , I4I; sex-limited, $2 S_{7}$

Insanity, 283

Insects, work with, 2 I 2

Ishmaelites, the, pedigree of, 307

Johannsen, W. L., work of, 85 , i 10

Jukes, the, pedigree of, 305

Kammerer, work of, 2 I I

Kentucky aristocracy, pedigree of, 304

Klebs, G., work of, 204

Knight, Thomas, work of, 84

Kölreuter, work of, 84

Lamarck, work of, ro

Latency, 97

Lee, Francis Lightfoot, pedigree of, 303
Lee, Richard, pedigree of, 303

Lee, Richard Henry, pedigree of, 303

Lee, Robert E., pedigree of, 303

Leptinotarsa, work with, I81, 216

Lutz, work of, 213

MacDougal, D. T., work of, 205,222

Macro-gamete, 64

Maize, work with, 54,85 , 1 16

Man, inheritance of physical and mental traits of, 269

Manhattan Island and eugenics, 296

Mendel, Gregor, work of, is, 40

Mendelism, I8, 40, 85, I00, I04

Mice, work with, 2 I 2

Micro-gamete, 64

Migrations, and their eugenic significance, 294

Minot, Charles Sedwick, pedigree of, 302

Monohybrids, 9I

Morgan, T. H., work of, 75, 222

Mutation, I3, I 70

Naegeli, Karl, work of, 40

Natural selection, I I

Neo-Darwinians, 168

Nereis, work with, 47

New England and eugenics, 296

Nitrogen, in soil, 120

Nucleus, 24

Oenothera, work with, I72, 197

Oranges, work with, 128

Orthogenesis, 14

Orton, W. A., work of, 125

Paine, Robert Treat, pedigree of, 302

Pangenesis, I 42

Parthenogenesis, 67

Patton, Elizabeth, pedigree of, 304

Pauperism, 285

Pearson, Karl, work of, 16

Phosphorus, in soil, I 20

Plant breeding, I 13

Potassium, in soil, 120 
Preston, John, pedigree of, 304

Price, H. I.., work of, I 25

Pringsheim, N., work of, 203

Protoplast, 24

Punnett, R. C., work of, 66

Reeve, Sarah Tapping, pedigree of, zor

Reichert, work of, 224

Reproduction, power of, 23; sexual, 29; by spores, 29

Richardson, Ann, pedigree of, 302

Riley, C. V., work of, 65

Russo, work of, 65

St. Hilaire, Geoffrey, work of, 9

Saltation, I 7 I

Schenk, work of, 65

Sedgwick, Catherine Maria, pedigree of, 302

Selection, 118 , 131

Sempervivum, work with, 204

Sex, and heredity, 62 ; immorality, $28_{5}$

Shamel, A. D., work of, 124

Skeleton, inherited characters of, 277

Soil, I 20

Sparks, Jared, pedigree of, zor

Sperm, 3I, 45

Sporophy'te, 34

Stoughton, Abigail, pedigree of, 302

Sumner, F. B., work of, $210,2 \mathrm{r} 2$

Swimming spores, 29

Synthesis of a mutating race, $r \delta_{2}$

Talcott, Mary, pedigree of, $30 \mathrm{I}$

Thomsen's disease, 278

Tobacco, work with, II 4

Tower, William Lawrence, $\mathrm{r}_{4} \mathrm{r}$

Treat, Mary, work of, 65
Tschermak, li., work of, $f$ I

Tuttle, Elizabeth, perligree of, 300

Tyler, 1)aniel, pedigree of, zor

Unit-character, 48

Use and disuse, 10

Variation, germinal, in animals, 210; in Chrysomelid beetles, 213; by combined selection and hybridization, 234; by different forces, 200; by hybridization, 23I; origin of, 144; peripheral origin of, $x+3$; in plants, 202 ; by selection, 238 ; somatic, I 45

Vilmorin, P., work of, $8+$

Vincent, Marvin Richardson, pedigree of, 302

Virginia and eugenics, 295; first families, of 303

Von Rümker, work of, I 25

Waite, Morrison, pedigree of, 302

Washington, George, pedigree of, 304

Webber, H., work of, r25, r 28

Weismann, A., work of, 4I, IIO, I6S

Whitney, Henrietta Frances, pedigree of, 302

IVilson, E. B., work of, 47

Woltereck, work of, 2 IO

Woolsey, Theodore Dwight, pedigree of, 301

Yeasts, work with, 202

Zedebauer, E., work of, 203

Zoospore, 29

Zygote, 30, 62 
ins 47 




\section{PLEASE DO NOT REMOVE \\ CARDS OR SLIPS FROM THIS POCKET \\ UNIVERSITY OF TORONTO LIBRARY}

EloMed 
